\title{
Enantioselective Synthesis of Spirorhodanine-Pyran Derivatives via \\ Organocatalytic [3+3] Annulation Reactions between Pyrazolones and Rhodanine-Derived Ketoesters
}

Dong-Sheng Ji, Yong-Chun Luo, Xiu-Qin Hu, and Peng-Fei Xu*

State Key Laboratory of Applied Organic Chemistry, College of Chemistry and Chemical Engineering, Lanzhou University, Lanzhou 730000, P.R. China

\section{Contents}

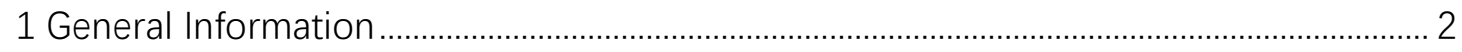

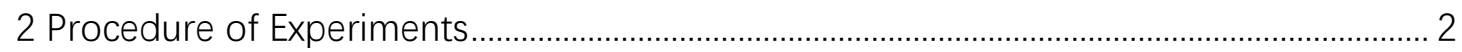

2.1 General Procedure for the Synthesis of Rhodanine-Derived Ketoesters .................... 2

2.2 General Procedure of the [3+3] Annulation Reaction ................................................. 3

2.3 Procedure for the Derivatization of 3aa...................................................................... 3

2.4 The Procedure of the Gram-scale Asymmetric Synthesis of 3aa................................. 4

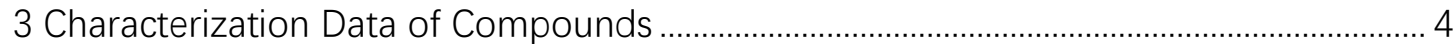

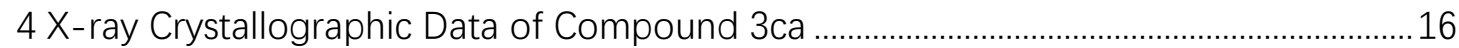

5 The Discussion and Determination of Absolute Configuration of Compound ................17

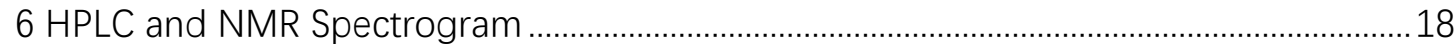




\section{General Information}

Chemicals and solvents were either purchased from commercial suppliers or purified by standard techniques. Analytical thin-layer chromatography (TLC) was performed on silica gel plates with F-254 indicator and compounds were visualized by irradiation with UV light. Flash chromatography was carried out utilizing silica gel 200-300 mesh. ${ }^{1} \mathrm{H}$ NMR, ${ }^{13} \mathrm{C}$ NMR spectra were recorded on a 400 spectrometer $\left(400 \mathrm{MHz}{ }^{1} \mathrm{H}, 100 \mathrm{MHz}{ }^{13} \mathrm{C}\right.$ ) or a 600 spectrometer $\left(600 \mathrm{MHz}{ }^{1} \mathrm{H}, 150 \mathrm{MHz}{ }^{13} \mathrm{C}\right)$. The spectra were recorded in $\mathrm{CDCl}_{3}$ as the solvent at room temperature, ${ }^{1} \mathrm{H}$ and ${ }^{13} \mathrm{CNMR}$ chemical shifts are reported in ppm relative to either the residual solvent peak $\left({ }^{13} \mathrm{C}\right)(\delta=77.00 \mathrm{ppm})$ or TMS $\left({ }^{1} \mathrm{H}\right)(\delta=0 \mathrm{ppm})$ as an internal standard. Data for ${ }^{1} \mathrm{H}$ NMR are reported as follows: chemical shift $(\delta \mathrm{ppm})$, multiplicity ( $\mathrm{s}=$ singlet, $\mathrm{d}=$ doublet, $\mathrm{t}=$ triplet, $\mathrm{m}=$ multiplet, $\mathrm{br}=$ broad $)$, integration, coupling constant $(\mathrm{Hz})$ and assignment. Data for ${ }^{13} \mathrm{C}$ NMR are reported as chemical shift. HRMS were performed on mass instrument (ESI). Enantiomeric excess values were determined by HPLC with Chirapak IA or IF column on Agilent 1260 series with $i$-PrOH and $n$-hexane. Optical rotation was measured on the Perkin Elmer 341 polarimeter with $[\alpha]_{\mathrm{D}}$ values reported in degrees. Concentration (c) is in $\mathrm{g} / 100 \mathrm{~mL}$.

\section{Procedure of Experiments}

\subsection{General Procedure for the Synthesis of Rhodanine-Derived Ketoesters}

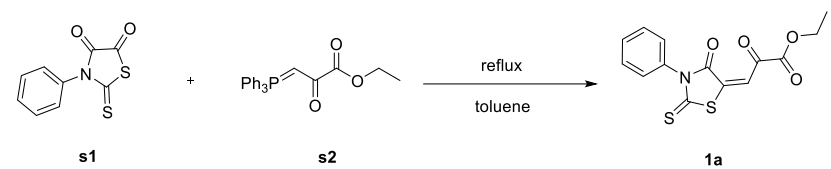

In a $50 \mathrm{~mL}$ round-bottomed flask, $\mathbf{s 1}^{1}(10 \mathrm{mmol})$ and phosphoranes $\mathbf{s 2}^{2}(12 \mathrm{mmol})$ were dissolved in 20 $\mathrm{mL}$ of toluene, and the mixture was refluxed for $1.5 \mathrm{~h}$. Then the mixture was cooled down to room temperature, the solvent was removed on a rotary evaporator, the residue was purified by silica gel flash column chromatography (Petroleumether/EtOAc=10:1 t) to give the corresponding compound as a red solid.

\footnotetext{
Ritter, W. Tetrahedron Lett. 1967, 46, 4593-4596

${ }^{2}$ Charles S. Craik. Bioorg. Med. Chem., 2005, 13, 2141-2156
} 


\subsection{General Procedure of the [3+3] Annulation Reaction}
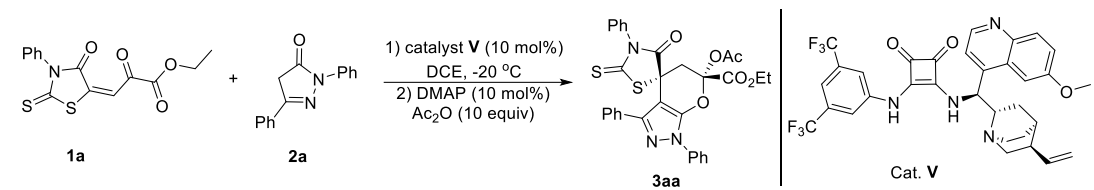

To a test tube (flame dried) with a stirrer were successively added rhodanine-derived ketoester $\mathbf{1}(0.1$ mmol, $32.1 \mathrm{mg})$, pyrazolone $2(0.2 \mathrm{mmol}, 47.2 \mathrm{mg})$, catalyst $\mathbf{V} .(0.01 \mathrm{mmol}, 6.3 \mathrm{mg})$ and dried DCE (precooled to $\left.-20{ }^{\circ} \mathrm{C}\right)(1 \mathrm{~mL})$ at $-20{ }^{\circ} \mathrm{C}$. When TLC analysis showed that $\mathbf{1}$ was completely consumed, acetic anhydride $(0.1 \mathrm{~mL})$ and DMAP $(0.01 \mathrm{mmol}, 1.3 \mathrm{mg})$ were added, and the mixture was stirred for a further 0.5 hour at room temperature. After that, the solvent was evaporated under reduced pressure and the residue was purified by silica gel flash column chromatography (Petroleumether/EtOAc=10:1 to 5:1) to give the corresponding compound 3aa. Chiral 3ab-3sa were synthesized using the same method.

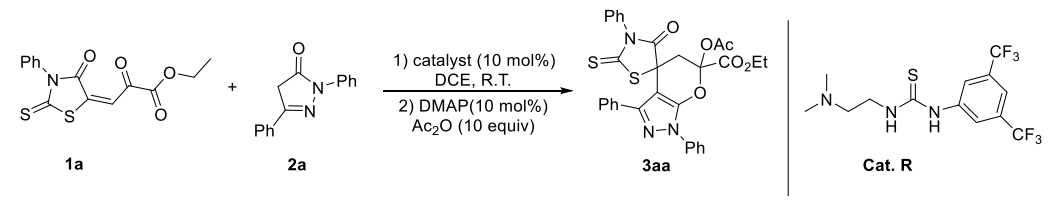

To a test tube (flame dried) with a stirrer were successively added rhodanine-derived ketoester $1(0.1 \mathrm{mmol}, 32.1 \mathrm{mg})$, pyrazolone $2(0.1 \mathrm{mmol}, 23.6 \mathrm{mg})$, cat. $\mathbf{R}(0.1 \mathrm{mmol}, 3.8 \mathrm{mg})$ and dried DCE $(1 \mathrm{~mL})$, the mixture was stirred for 12 hour at room temperature. Then acetic anhydride $(0.1 \mathrm{~mL})$ and DMAP $(0.01 \mathrm{mmol}, 1.32 \mathrm{mg})$ were added, the mixture was stirred for a further 0.5 hour at room temperature. After that, the solvent was evaporated under reduced pressure and the residue was purified by silica gel flash column chromatography (Petroleumether/EtOAc=10:1 to 5:1) to give the corresponding racemic compounds 3aa. Racemic 3ab-3sa were synthesized using the same method.

\subsection{Procedure for the Derivatization of 3aa.}

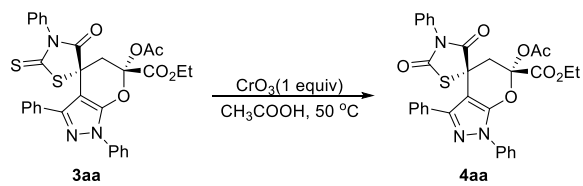

In a $10 \mathrm{~mL}$ test tube (flame dried) with a stirrer were successively added $\mathbf{3 a a}(0.1 \mathrm{mmol}, 60 \mathrm{mg}), \mathrm{CrO}_{3}$ $(0.1 \mathrm{mmol}, 10 \mathrm{mg})$ and $1 \mathrm{ml}$ acetic acid, the mixture was warmed up to $50{ }^{\circ} \mathrm{C}$ and stirred for 12 hours. The mixture was then cooled down to room temperature, saturated ammonium chloride aqueous solution $(10 \mathrm{~mL})$ was added to the mixture. Then this mixtured was extracted three times with $10 \mathrm{~mL}$ DCM $(10 \mathrm{~mL} * 3)$, the organic layers were combined, dried over $\mathrm{Na}_{2} \mathrm{SO}_{4}$, filtered and concentrated. The 
resulting residue was purified by flash column chromatography (Petroleumether/EtOAc= 5:1) on silica gel to give the corresponding compound 4aa.

\subsection{The Procedure of the Gram-scale Asymmetric Synthesis of 3aa}

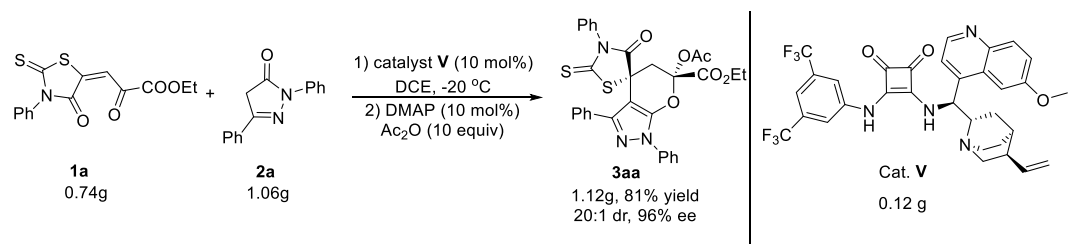

In a $25 \mathrm{~mL}$ round-bottomed flame dried flask, rhodanine-derived ketoester 1 (2.3 mmol, 0.74g), pyrazolone 2 (4.6 mmol, $1.08 \mathrm{~g})$ and cat. V. (0.1 mmol, 0.12g) were successively added, $23 \mathrm{~mL}$ DCE (precooled to $-20{ }^{\circ} \mathrm{C}$ ) was added by syringe, and the mixture was stirred for 72 hours at $-20{ }^{\circ} \mathrm{C}$. Then acetic anhydride $(2 \mathrm{~mL})$ and DMAP $(0.2 \mathrm{mmol}, 24 \mathrm{mg})$ were added, the mixture was stirred for a further 0.5 hour at room temperature. After that, the solvent was evaporated under reduced pressure and the residue was purified by silica gel flash column chromatography (Petroleumether/EtOAc=10:1 to $5: 1)$ to give the corresponding compound 3aa.

\section{Characterization Data of Compounds}

Ethyl(4R,6R)-6-acetoxy-4'-oxo-1,3,3'-triphenyl-2'-thioxo-5,6-dihydro-1H-spiro[pyrano[2,3-c] pyrazole-4,5'-thiazolidine]-6-carboxylate

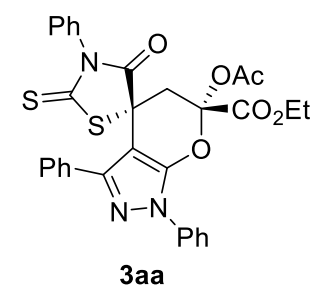

An amorphous solid, $85 \%$ yield $(51 \mathrm{mg}) .[\alpha]_{\mathrm{D}}{ }^{20}=22\left(c 1.0, \mathrm{CH}_{2} \mathrm{Cl}_{2}, 99 \%\right.$ ee); ${ }^{1} \mathrm{H} \mathrm{NMR}\left(400 \mathrm{MHz}, \mathrm{CDCl}_{3}\right): \delta=7.81-7.79(\mathrm{~m}, 2 \mathrm{H}), 7,73-7.63(\mathrm{~m}$, 2H), 7.42-7.49(m, 5H), 7.25-7.40(m, 4H), 5.59-6.96(br, 2H), 4.36(q, $J=$ $7.2 \mathrm{~Hz} 2 \mathrm{H}), 3.11(\mathrm{~d}, J=14.4 \mathrm{~Hz}, 1 \mathrm{H}), 3.07(\mathrm{~d}, J=14.4 \mathrm{~Hz}, 1 \mathrm{H}), 2.26(\mathrm{~s}$, $3 \mathrm{H}), 1.34(\mathrm{t}, J=7.2 \mathrm{~Hz}, 3 \mathrm{H}) ;{ }^{13} \mathrm{C} \mathrm{NMR}\left(100 \mathrm{MHz}, \mathrm{CDCl}_{3}\right): \delta=13.8$, 20.7, 41.1, 53.0, 63.3, 95.1, 96.7, 120.6, 126.8, 127.8, 128.5, 128.9, 129.0, 129.1, 129.2, 129.5, 132.6, 134.5, 137.4, 147.2, 148.6, 164.3, 167.7, 174.8, 199.1. The enantiomeric excess was determined by HPLC with an IA column. ( $n$-hexane:i-PrOH $=90: 10), 1 \mathrm{~mL} / \mathrm{min}$; major enantiomer $t_{R}=11.42$ min, minor enantiomer $t_{R}=19.81 \mathrm{~min}$. HRMS $(E S I):[M+H]^{+}$calcd for $\left[\mathrm{C}_{31} \mathrm{H}_{26} \mathrm{~N}_{3} \mathrm{O}_{6} \mathrm{~S}_{2}\right]$ : 599.1969, found: 599.1960 .

\section{Ethyl}

(4R,6R)-6-acetoxy-3' -(4-fluorophenyl)-4'-oxo-1,3-diphenyl-2'-thioxo-5,6-dihydro-1H-spiro[p yrano[2,3-c]pyrazole-4,5'-thiazolidine]-6-carboxylate

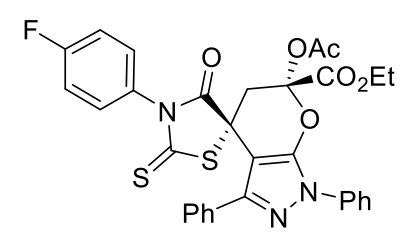

$3 a b$
An amorphous solid, 86\% yield $(53 \mathrm{mg}) .[\alpha]_{\mathrm{D}}^{20}=157$ (c 1.0, $\mathrm{CH}_{2} \mathrm{Cl}_{2}, 91 \%$ ee); ${ }^{1} \mathrm{H}$ NMR $\left(600 \mathrm{MHz}, \mathrm{CDCl}_{3}\right): \delta=7.85(\mathrm{~d}, J=$ $7.8 \mathrm{~Hz}, 2 \mathrm{H}), 7.65-7.49(\mathrm{~m}, 2 \mathrm{H}), 7.42-7.49(\mathrm{~m}, 5 \mathrm{H}), 7.29-7.33(\mathrm{~m}$, 
$1 \mathrm{H}), 6.98-7.05(\mathrm{~m}, 2 \mathrm{H}), 5.66-6.77(\mathrm{br}, 2 \mathrm{H}), 4.38(\mathrm{q}, J=7.2 \mathrm{~Hz}, 2 \mathrm{H}), 3.10(\mathrm{~d}, J=14.4 \mathrm{~Hz}, 1 \mathrm{H})$, 3.07(d, $J=14.4 \mathrm{~Hz}, 1 \mathrm{H}), 2.28(\mathrm{~s}, 3 \mathrm{H}), 1.36(\mathrm{t}, J=7.2 \mathrm{~Hz}, 3 \mathrm{H}) ;{ }^{13} \mathrm{C} \mathrm{NMR}\left(150 \mathrm{MHz}, \mathrm{CDCl}_{3}\right): \delta$ $=199.1,174.8,167.7,164.3,163.9,162.7(\mathrm{~d}, J=248 \mathrm{~Hz}), 148.5,147.2,137.4,132.7,130.1$ (d, $J=3 \mathrm{~Hz}), 129.9(\mathrm{~d}, J=9 \mathrm{~Hz}), 129.2,129.1,129.0,128.6,126.9,120.7,116.3(\mathrm{~d}, J=23 \mathrm{~Hz})$, 96.7, 95.1, 63.4, 52.9, 41.1, 20.8, 13.9. The enantiomeric excess was determined by HPLC with an IA- column. ( $n$-hexane: $i-\mathrm{PrOH}=90: 10), 1 \mathrm{~mL} / \mathrm{min}$; major enantiomer $\mathrm{t}_{\mathrm{R}}=8.92 \mathrm{~min}$, minor enantiomer $t_{R}=24.01 \mathrm{~min}$. HRMS (ESI): $[\mathrm{M}+\mathrm{H}]^{+}$calcd for $\left[\mathrm{C}_{31} \mathrm{H}_{25} \mathrm{FN}_{3} \mathrm{O}_{6} \mathrm{~S}_{2}\right]$ : 618.1163, found: 618.1161 .

Ethyl

(4R,6R)-6-acetoxy-3' -(4-chlorophenyl)-4'-oxo-1,3-diphenyl-2'-thioxo-5,6-dihydro-1H-spiro[p yrano[2,3-c]pyrazole-4,5'-thiazolidine]-6-carboxylate

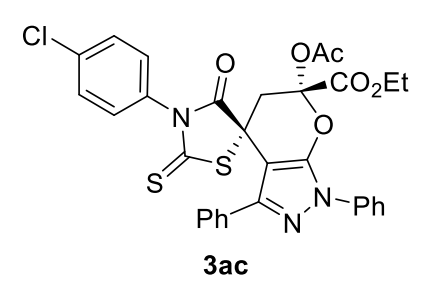

An amorphous solid, $85 \%$ yield $(54 \mathrm{mg}) \cdot[\alpha]_{\mathrm{D}}{ }^{20}=16(c \quad 1.0$, $\mathrm{CH}_{2} \mathrm{Cl}_{2}, 92 \%$ ee); ${ }^{1} \mathrm{H}$ NMR (600 MHz, $\left.\mathrm{CDCl}_{3}\right): \delta=7.83-7.88(\mathrm{~m}$, $2 \mathrm{H}), 7.64-7.68(\mathrm{~m}, 2 \mathrm{H}), 7.42-7.28(\mathrm{~m}, 5 \mathrm{H}), 7.28-7.33(\mathrm{~m}, 3 \mathrm{H})$, $5.70-6.78(\mathrm{br}, 2 \mathrm{H}), 4.38(\mathrm{q}, J=7.2 \mathrm{~Hz}, 2 \mathrm{H}), 3.10(\mathrm{~d}, J=14.4 \mathrm{~Hz}$, $1 \mathrm{H}), 3.06(\mathrm{~d}, J=14.4 \mathrm{~Hz}, 1 \mathrm{H}), 2.28(\mathrm{~s}, 3 \mathrm{H}), 1.36(\mathrm{t}, J=7.2 \mathrm{~Hz}, 3 \mathrm{H})$;

${ }^{13} \mathrm{C}$ NMR $\left(150 \mathrm{MHz}, \mathrm{CDCl}_{3}\right): \delta=198.9,174.7,167.7,164.4,148.6,147.4,137.5,135.7$, $132.9,132.8,129.5,129.3,129.2,129.1,128.7,126.9,120.7,96.8,95.2,63.4,55.0,53.1,41.2$, 20.8, 13.9. The enantiomeric excess was determined by HPLC with an IA- column. (n-hexane:i-PrOH = 90:10), $1 \mathrm{~mL} / \mathrm{min}$; major enantiomer $\mathrm{t}_{\mathrm{R}}=10.52 \mathrm{~min}$, minor enantiomer $\mathrm{t}_{\mathrm{R}}$ $=15.42$ min. HRMS (ESI): $[\mathrm{M}+\mathrm{H}]^{+}$calcd for $\left[\mathrm{C}_{31} \mathrm{H}_{25} \mathrm{ClN}_{3} \mathrm{O}_{6} \mathrm{~S}_{2}\right]: 634.0868$, found: 634.0864 .

\section{Ethyl}

(4R,6R)-6-acetoxy-4'-oxo-1,3-diphenyl-2'-thioxo-3'-(p-tolyl)-5,6-dihydro-1H-spiro[pyrano[2, 3-c]pyrazole-4,5'-thiazolidine]-6-carboxylate

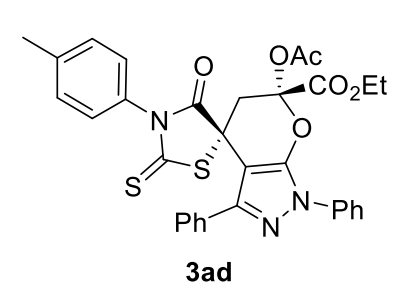

An amorphous solid, $83 \%$ yield $(51 \mathrm{mg}) .[\alpha]_{\mathrm{D}}^{20}=-11\left(c 1.0, \mathrm{CH}_{2} \mathrm{Cl}_{2}\right.$, $94 \%$ ee); ${ }^{1} \mathrm{H}$ NMR $\left(600 \mathrm{MHz}, \mathrm{CDCl}_{3}\right): \delta=7.49-7.89(\mathrm{~m}, 2 \mathrm{H})$, $7.65-7.69(\mathrm{~m}, \quad 2 \mathrm{H}), \quad 7.42-7.49(\mathrm{~m}, \quad 5 \mathrm{H}), \quad 7.28-7.32(\mathrm{~m}, \quad 1 \mathrm{H})$, 7.11-7.17(m, 2H), 5.88-6.56(br, 2H), 4.37(q, $J=7.2 \mathrm{~Hz}, 2 \mathrm{H})$, $3.11(\mathrm{~d}, J=14.4 \mathrm{~Hz}, 1 \mathrm{H}), 3.07(\mathrm{~d}, J=14.4 \mathrm{~Hz}, 1 \mathrm{H}), 2.34(\mathrm{~s}, 3 \mathrm{H})$, 2.28(s, 3H), 1.36(t, J=7.2 Hz, 3H); ${ }^{13} \mathrm{C}$ NMR (100 MHz, $\left.\mathrm{CDCl}_{3}\right)$ : 199.4, 174.9, 167.7, 164.4, $148.7,147.3,139.8,137.5,132.7,131.9,129.9,129.2$, 129.1, 129.0, 128.6, 127.6, 126.8, $120.7,96.8,95.3,63.4,55.0,53.0,41.2,21.2,13.9$. The enantiomeric excess was determined by HPLC with an IA- column. (n-hexane: $i-\mathrm{PrOH}=90: 10), 1 \mathrm{~mL} / \mathrm{min}$; major enantiomer $\mathrm{t}_{\mathrm{R}}=$ 
8.60 min, minor enantiomer $t_{R}=15.01$ min. HRMS (ESI): $[M+H]^{+}$calcd for $\left[\mathrm{C}_{32} \mathrm{H}_{28} \mathrm{~N}_{3} \mathrm{O}_{6} \mathrm{~S}_{2}\right]$ : 614.1414, found: 614.1413 .

\section{Ethyl}

(4R,6R)-6-acetoxy-3'-(4-methoxyphenyl)-4'-oxo-1,3-diphenyl-2'-thioxo-5,6-dihydro-1H-spiro [pyrano[2,3-c]pyrazole-4,5'-thiazolidine]-6-carboxylate

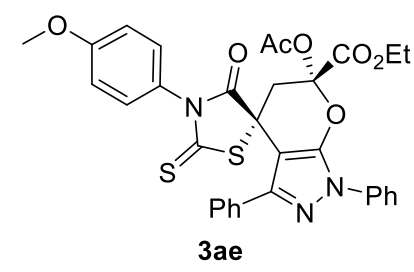

An amorphous solid, $85 \%$ yield $(53 \mathrm{mg})$. m. p.: $187-191^{\circ} \mathrm{C}[\alpha]_{\mathrm{D}}{ }^{20}$ $=\left(c\right.$ 1.0, $\mathrm{CH}_{2} \mathrm{Cl}_{2}, 90 \%$ ee $) ;{ }^{1} \mathrm{H} \mathrm{NMR}\left(400 \mathrm{MHz}, \mathrm{CDCl}_{3}\right): \delta=$ 7.94-7.81(d, $J=8 \mathrm{~Hz}, 2 \mathrm{H}), 7.64-7.70(\mathrm{~m}, 2 \mathrm{H}), 7.41-7.51(\mathrm{~m}, 5 \mathrm{H})$, 7.27-7.35(m, 1H), 6.78-6.86(m, 2H), 5.78-6.82(br, 2H), 4.37(q, $J$ $=7.2 \mathrm{~Hz}, 2 \mathrm{H}), 3.78(\mathrm{~s}, 3 \mathrm{H}), 3.11(\mathrm{~d}, J=14.8 \mathrm{~Hz}, 1 \mathrm{H}), 3.06(\mathrm{~d}, J=$ $14.8 \mathrm{~Hz}, 1 \mathrm{H}), 2.27(\mathrm{~s}, 3 \mathrm{H}), 1.35(\mathrm{t}, J=7.2 \mathrm{~Hz}, 3 \mathrm{H}) ;{ }^{13} \mathrm{C} \mathrm{NMR}\left(100 \mathrm{MHz}, \mathrm{CDCl}_{3}\right): \delta=199.5$, 174.9, 167.7, 164.3, 160.0, 148.6, 147.3, 137.4, 132.7, 129.2, 129.1, 129.0, 128.6, 126.9, $126.8,120.6,114.4,96.8,95.2,63.4,55.3,52.8,41.1,20.8,13.8$. The enantiomeric excess was determined by HPLC with an IA- column. (n-hexane: $i-\mathrm{PrOH}=90: 10), 1 \mathrm{~mL} / \mathrm{min}$; major enantiomer $t_{R}=12.39$ min, minor enantiomer $t_{R}=23.89$ min. HRMS $(E S I):[M+H]^{+}$calcd for $\left[\mathrm{C}_{32} \mathrm{H}_{28} \mathrm{~N}_{3} \mathrm{O}_{7} \mathrm{~S}_{2}\right]$ : 630.1363, found: 630.1365 .

\section{Ethyl}

(4R,6R)-6-acetoxy-3-(4-fluorophenyl)-4'-oxo-1,3'-diphenyl-2'-thioxo-5,6-dihydro-1H-spiro[p yrano[2,3-c]pyrazole-4,5'-thiazolidine]-6-carboxylate

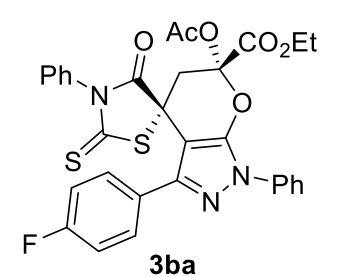

An amorphous solid, 89\% yield (55 mg). $[\alpha]_{\mathrm{D}}^{20}=-17\left(c 1.0, \mathrm{CH}_{2} \mathrm{Cl}_{2}, 94 \%\right.$ ee); ${ }^{1} \mathrm{H}$ NMR $\left(600 \mathrm{MHz}, \mathrm{CDCl}_{3}\right): \delta=7.84(\mathrm{~d}, J=7.8 \mathrm{~Hz} 2 \mathrm{H})$, 7.64-7.70(m, 2H), 7.44-7.51(m, 2H), 7.36-7.43(m, 3H), 7.29-7.33(m, 1H), 7.13-7.20(m, 2H), 5.72-6.98(br, 2H), 4.48(q, $J=7.2 \mathrm{~Hz}, 2 \mathrm{H}), 3.10$ $(\mathrm{t}, J=16 \mathrm{~Hz}, 2 \mathrm{H}), 2.28(\mathrm{~s}, 3 \mathrm{H}), 1.36(\mathrm{t}, J=7.2 \mathrm{~Hz}, 3 \mathrm{H}) ;{ }^{13} \mathrm{C}$ NMR $(150$ $\left.\mathrm{MHz}, \mathrm{CDCl}_{3}\right): \delta=198.9,174.8,167.7,163.4(\mathrm{~d}, J=247 \mathrm{~Hz}), 147.6,147.4,137.4,134.5$, 130.6, 130.5, 129.7, 129.3, 129.2, 128.8, 127.8, 126.9, 120.7, 116.1(d, $J=15 \mathrm{~Hz}), 96.8,95.2$, $63,4,53.0,41.2,20.8,13.9$. The enantiomeric excess was determined by HPLC with an IAcolumn. (n-hexane: $i-\mathrm{PrOH}=90: 10$ ), $1 \mathrm{~mL} / \mathrm{min}$; major enantiomer $\mathrm{t}_{\mathrm{R}}=8.16 \mathrm{~min}$, minor enantiomer $t_{R}=14.66$ min. HRMS (ESI): $[\mathrm{M}+\mathrm{H}]^{+}$calcd for $\left[\mathrm{C}_{31} \mathrm{H}_{25} \mathrm{FN}_{3} \mathrm{O}_{6} \mathrm{~S}_{2}\right]: 618.1161$, found: 618.1163 .

\section{Ethyl}

(4R,6R)-6-acetoxy-3-(4-bromophenyl)-4'-oxo-1,3'-diphenyl-2'-thioxo-5,6-dihydro-1H-spiro[p yrano[2,3-c]pyrazole-4,5'-thiazolidine]-6-carboxylate 


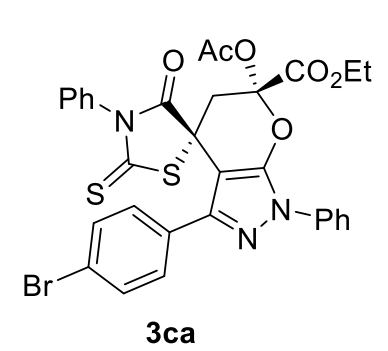

An amorphous solid, $84 \%$ yield $(57 \mathrm{mg})$. m. p.: $154-157^{\circ} \mathrm{C}[\alpha]_{\mathrm{D}}{ }^{20}=8$ (c 1.0, $\mathrm{CH}_{2} \mathrm{Cl}_{2}, 96 \%$ ee); ${ }^{1} \mathrm{H}$ NMR $\left(600 \mathrm{MHz}, \mathrm{CDCl}_{3}\right): \delta=$ 7.82-7.85(m, 2H), 7.58-7.61(m, 2H), 7.53-7.57(m, 2H), 7.45-7.49(m, $2 \mathrm{H}), 7.39-7.43(\mathrm{~m}, 3 \mathrm{H}), 7.30-7.33(\mathrm{~m}, 1 \mathrm{H}), 5.78-6.88(\mathrm{br}, 2 \mathrm{H}), 4.38(\mathrm{q}$, $J=7.2 \mathrm{~Hz}, 2 \mathrm{H}), 3.09(\mathrm{~s}, 2 \mathrm{H}), 2.28(\mathrm{~s}, 3 \mathrm{H}), 1.36(\mathrm{t}, J=7.2 \mathrm{~Hz}, 3 \mathrm{H})$;

${ }^{13} \mathrm{C} \mathrm{NMR}\left(150 \mathrm{MHz}, \mathrm{CDCl}_{3}\right): \delta=198.8,174.7,167.6,164.3,147.5$, 137.4, 134.5, 132.2, 131.7, 130.3, 129.7, 129.4, 129.3, 127.8, 127.0, 123.5, 120.7, 96.8, 95.4, 63.4, 52.9, 41.2, 20.8, 13.9. The enantiomeric excess was determined by HPLC with an IAcolumn. (n-hexane: $i-\mathrm{PrOH}=90: 10), 1 \mathrm{~mL} / \mathrm{min}$; major enantiomer $t_{\mathrm{R}}=8.49 \mathrm{~min}$, minor enantiomer $t_{R}=15.67$ min. HRMS (ESI): $[\mathrm{M}+\mathrm{H}]^{+}$calcd for $\left[\mathrm{C}_{31} \mathrm{H}_{25} \mathrm{BrN}_{3} \mathrm{O}_{6} \mathrm{~S}_{2}\right]: 678.0363$, 680.0342, found: $678.0361,680.0339$.

\section{Ethyl}

(4R,6R)-6-acetoxy-3-(4-methoxyphenyl)-4'-oxo-1,3'-diphenyl-2'-thioxo-5,6-dihydro-1H-spiro [pyrano[2,3-c]pyrazole-4,5'-thiazolidine]-6-carboxylate

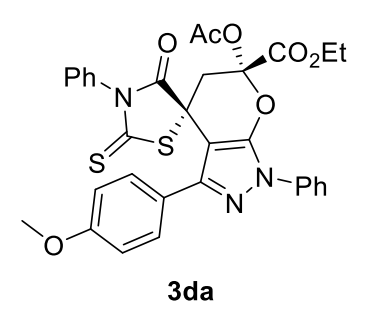

An amorphous solid, $71 \%$ yield $(48 \mathrm{mg}) \cdot[\alpha]_{\mathrm{D}}^{20}=-9\left(c 1.0, \mathrm{CH}_{2} \mathrm{Cl}_{2}, 90 \%\right.$ ee); ${ }^{1} \mathrm{H}$ NMR $\left(600 \mathrm{MHz}, \mathrm{CDCl}_{3}\right): \delta=7.83-7.88(\mathrm{~m}, 2 \mathrm{H}), 7.58-7.62(\mathrm{~m}$, $2 \mathrm{H}), \quad 7.44-7.48(\mathrm{~m}, \quad 2 \mathrm{H}), \quad 7.33-7.41(\mathrm{~m}, \quad 3 \mathrm{H}), \quad 7.28-7.32(\mathrm{~m}, \quad 1 \mathrm{H})$, 6.95-6.49(m, 2H), 5.75-6.79(br, 2H), 4.38(q, $J=7.2 \mathrm{~Hz}, 2 \mathrm{H}), 3.83(\mathrm{~s}$, $3 \mathrm{H}), 3.10(\mathrm{~d}, J=14.4 \mathrm{~Hz}, 1 \mathrm{H}), 3.07(\mathrm{~d}, J=14.4 \mathrm{~Hz}, 1 \mathrm{H}), 2.28(\mathrm{~s}, 3 \mathrm{H})$, $1.36(\mathrm{t}, J=7.2 \mathrm{~Hz}, 3 \mathrm{H}) ;{ }^{13} \mathrm{C} \mathrm{NMR}\left(150 \mathrm{MHz}, \mathrm{CDCl}_{3}\right): \delta=199.2,174.9,167.7,164.4,160.4$, 148.5, 147.3, 137.6, 134.6, 129.9, 129.6, 129.2, 129.1, 128.0, 126.7, 125.1, 120.6, 114.6, 96.9, 95.1, 63.4, 55.4, 55.4, 53.2, 41.3, 20.8, 13.9. The enantiomeric excess was determined by HPLC with an IA- column. ( $n$-hexane: $i-\operatorname{PrOH}=90: 10), 1 \mathrm{~mL} / \mathrm{min}$; major enantiomer $t_{\mathrm{R}}=$ 11.09 min, minor enantiomer $t_{R}=20.09$ min. HRMS (ESI): $[\mathrm{M}+\mathrm{H}]^{+}$calcd for $\left[\mathrm{C}_{32} \mathrm{H}_{28} \mathrm{~N}_{3} \mathrm{O}_{7} \mathrm{~S}_{2}\right]$ : 630.1363, found: 630.1363 .

\section{Ethyl}

(4R,6R)-6-acetoxy-4'-oxo-1,3'-diphenyl-2'-thioxo-3-(4-(trifluoromethyl)phenyl)-5,6-dihydro$1 \mathrm{H}$-spiro[pyrano[2,3-c]pyrazole-4,5'-thiazolidine]-6-carboxylate

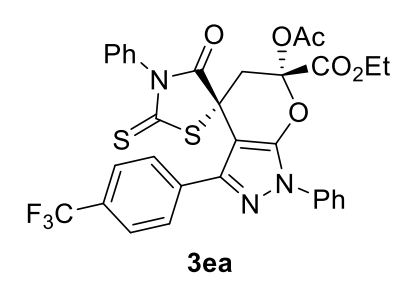

An amorphous solid, $68 \%$ yield $(46 \mathrm{mg}) .[\alpha]_{\mathrm{D}}^{20}=-15$ (c 1.0, $\mathrm{CH}_{2} \mathrm{Cl}_{2}, 90 \%$ ee); ${ }^{1} \mathrm{H} \mathrm{NMR}\left(400 \mathrm{MHz}, \mathrm{CDCl}_{3}\right): \delta=7.83-7.88(\mathrm{~m}$, $2 \mathrm{H}), \quad 7.78-7.82(\mathrm{~m}, 2 \mathrm{H}), \quad 7.69-7.76(\mathrm{~m}, 2 \mathrm{H}), 7.44-7.52(\mathrm{~m}, 2 \mathrm{H})$, 7.36-7.42(m, 1H), 7.29-7.33(m, 3H), 6.02-6.68(br, 2H), 4.39(q, $J=$

$7.2 \mathrm{~Hz}, 2 \mathrm{H}), 3.10(\mathrm{~s}, 2 \mathrm{H}), 2.28(\mathrm{~s}, 3 \mathrm{H}), 1.36(\mathrm{t}, J=7.2 \mathrm{~Hz}, 3 \mathrm{H}) ;{ }^{13} \mathrm{C}$ $\mathrm{NMR}\left(100 \mathrm{MHz}, \mathrm{CDCl}_{3}\right): \delta=198.6,174.7,167.7,164.2,147.6,147.1,137.3,136.4,134.3$, 
$131.3,130.9,129.8,129.4,129.3,129.0,127.6,127.1,126.0\left(\mathrm{~d}, J=3.63 \mathrm{~Hz}\right.$, for $\left.\mathrm{CF}_{3}-\mathrm{oC}\right)$, $120.7,96.8,95.5,63.5,52.9,41.2,20.8,13.9$. The enantiomeric excess was determined by HPLC with an IA- column. ( $n$-hexane: $i-\mathrm{PrOH}=90: 10), 1 \mathrm{~mL} / \mathrm{min}$; major enantiomer $\mathrm{t}_{\mathrm{R}}=$ $7.36 \mathrm{~min}$, minor enantiomer $\mathrm{t}_{\mathrm{R}}=14.46 \mathrm{~min}$. HRMS (ESI): $[\mathrm{M}+\mathrm{H}]^{+}$calcd for $\left[\mathrm{C}_{32} \mathrm{H}_{25} \mathrm{~F}_{3} \mathrm{~N}_{3} \mathrm{O}_{6} \mathrm{~S}_{2}\right]$ : 668.1130, found: 668.1131 .

\section{Ethyl}

(4R,6R)-6-acetoxy-4' -oxo-1,3'-diphenyl-2'-thioxo-3-(o-tolyl)-5,6-dihydro-1H-spiro[pyrano[2, 3-c]pyrazole-4,5'-thiazolidine]-6-carboxylate

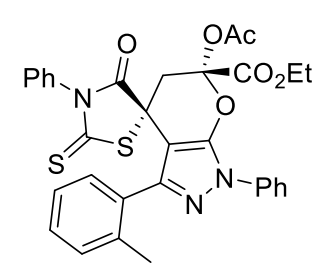

$3 \mathrm{fa}$

An amorphous solid, $87 \%$ yield $(53 \mathrm{mg}) \cdot[\alpha]_{\mathrm{D}}^{20}=41\left(c 1.0, \mathrm{CH}_{2} \mathrm{Cl}_{2}, 92 \%\right.$ ee); ${ }^{1} \mathrm{H}$ NMR $\left(600 \mathrm{MHz}, \mathrm{CDCl}_{3}\right): \delta=7.84(\mathrm{~d}, J=7.8 \mathrm{~Hz}, 2 \mathrm{H})$, 7.50-7.53(m, 1H), 7.43-7.48(m, 2H), 7.32-7.40(m, 5H), 7.27-7.31(m, $1 \mathrm{H}), 7.19-7.23(\mathrm{~m}, 1 \mathrm{H}), 5.78-6.86(\mathrm{br}, 2 \mathrm{H}), 4.38(\mathrm{q}, J=7.2 \mathrm{~Hz}, 2 \mathrm{H})$, $3.14(\mathrm{~d}, J=13.8 \mathrm{~Hz}, 1 \mathrm{H}), 3.05(\mathrm{~d}, J=13.8 \mathrm{~Hz}, 1 \mathrm{H}), 2.28(\mathrm{~s}, 6 \mathrm{H}), 1.37(\mathrm{t}, J$ $=7.2 \mathrm{~Hz}, 3 \mathrm{H}) ;{ }^{13} \mathrm{C}$ NMR $\left(150 \mathrm{MHz}, \mathrm{CDCl}_{3}\right): \delta=199.1,174.1,167.7,164.4,147.9,147.0$, 138.6, 137.6, 134.6, 131.5, 130.7, 129.8, 129.5, 129.2, 129.1, 128.1, 126.7, 126.1, 120.5, 97.0, 95.8, 63.4, 53.0, 40.9, 20.8, 20.0, 13.9. The enantiomeric excess was determined by HPLC with an IA- column. (n-hexane: $i-\mathrm{PrOH}=90: 10), 1 \mathrm{~mL} / \mathrm{min}$; major enantiomer $t_{\mathrm{R}}=7.24 \mathrm{~min}$, minor enantiomer $t_{R}=11.90$ min. HRMS (ESI): $[\mathrm{M}+\mathrm{H}]^{+}$calcd for $\left[\mathrm{C}_{32} \mathrm{H}_{28} \mathrm{~N}_{3} \mathrm{O}_{6} \mathrm{~S}_{2}\right]: 614.1414$, found: 614.1414 .

\section{Ethyl}

(4R,6R)-6-acetoxy-3-(2-chlorophenyl)-4'-oxo-1,3'-diphenyl-2'-thioxo-5,6-dihydro-1H-spiro[p yrano[2,3-c]pyrazole-4,5'-thiazolidine]-6-carboxylate

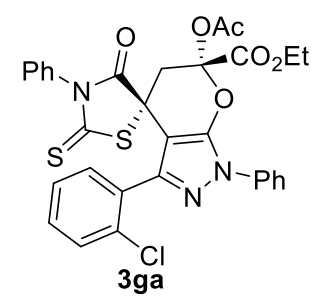

An amorphous solid, $68 \%$ yield $(43 \mathrm{mg}) \cdot[\alpha]_{\mathrm{D}}^{20}=42\left(c 1.0, \mathrm{CH}_{2} \mathrm{Cl}_{2}, 85 \%\right.$ ee); ${ }^{1} \mathrm{H}$ NMR (400 MHz, $\left.\mathrm{CDCl}_{3}\right): \delta=7.81-7.87(\mathrm{~m}, 2 \mathrm{H}), 7.54-7.59(\mathrm{~m}$, $2 \mathrm{H}), \quad 7.42-7.49(\mathrm{~m}, \quad 3 \mathrm{H}), \quad 7.34-7.39(\mathrm{~m}, \quad 3 \mathrm{H}), \quad 7.28-7.33(\mathrm{~m}, \quad 2 \mathrm{H})$, 6.18-6.59(br, 2H), 4.38(q, $J=7.2 \mathrm{~Hz}, 2 \mathrm{H}), 3.19(\mathrm{~d}, J=14.4 \mathrm{~Hz}, 1 \mathrm{H})$, $3.06(\mathrm{~d}, J=14.4 \mathrm{~Hz}, 1 \mathrm{H}), 2.27(\mathrm{~s}, 3 \mathrm{H}), 1.36(\mathrm{t}, J=7.2 \mathrm{~Hz}, 3 \mathrm{H}) ;{ }^{13} \mathrm{C} \mathrm{NMR}$ $\left(100 \mathrm{MHz}, \mathrm{CDCl}_{3}\right): \delta=199.0,174.5,167.7,164.3,147.0,145.4,137.4,135.0,134.5,131.7$, 131.1, 130.5, 130.2, 129.6, 129.2, 128.0, 127.0, 126.9, 120.8, 97.0, 96.1, 63.4, 52.8, 40.8, 20.8, 13.9. The enantiomeric excess was determined by HPLC with an IF- column. (n-hexane: $i$-PrOH $=90: 10$ ), $1 \mathrm{~mL} / \mathrm{min}$; major enantiomer $\mathrm{t}_{\mathrm{R}}=9.26 \mathrm{~min}$, minor enantiomer $\mathrm{t}_{\mathrm{R}}$ $=18.09$ min. HRMS (ESI): $[\mathrm{M}+\mathrm{H}]^{+}$calcd for $\left[\mathrm{C}_{31} \mathrm{H}_{25} \mathrm{ClN}_{3} \mathrm{O}_{6} \mathrm{~S}_{2}\right]: 634.0868$, found: 634.0865. 


\section{Ethyl}

(4R,6R)-6-acetoxy-4'-oxo-1,3'-diphenyl-2'-thioxo-3-(m-tolyl)-5,6-dihydro-1H-spiro[pyrano[2, 3-c]pyrazole-4,5'-thiazolidine]-6-carboxylate<smiles>CCOC(=O)C1(OC(C)=O)CC2(SC(=S)N(c3ccccc3)C2=O)c2c(-c3cccc(C)c3)nn(-c3ccccc3)c2O1</smiles>

An amorphous solid, $84 \%$ yield $(51 \mathrm{mg}) .[\alpha]_{\mathrm{D}}{ }^{20}=21$ (c 1.0, $\mathrm{CH}_{2} \mathrm{Cl}_{2}$, 94\% ee); ${ }^{1} \mathrm{H}$ NMR $\left(600 \mathrm{MHz}, \mathrm{CDCl}_{3}\right): \delta=7.86(\mathrm{~d}, J=7.8 \mathrm{~Hz}, 2 \mathrm{H})$, 7.44-7.50(m, 4H), 7.27-7.40(m, 6H), 5.63-6.97(br, 2H), 4.38(q, $J=$ $7.2 \mathrm{~Hz}, 2 \mathrm{H}), 3.11(\mathrm{~d}, J=14.4 \mathrm{~Hz}, 1 \mathrm{H}), 3.07(\mathrm{~d}, J=14.4 \mathrm{~Hz}, 1 \mathrm{H})$, $2.33(\mathrm{~s}, 3 \mathrm{H}), 2.28(\mathrm{~s}, 3 \mathrm{H}), 1.36(\mathrm{t}, J=7.2 \mathrm{~Hz}, 3 \mathrm{H}) ;{ }^{13} \mathrm{C}$ NMR $(150$ $\left.\mathrm{MHz}, \mathrm{CDCl}_{3}\right): \delta=199.1,174.9,167.7,164.4,148.9,147.3,139.0$,

137.6, 129.9, 129.6, 128.0, 126.8, 125.8, 120.7, 96.9, 95.2, 63.4, 53.2, 41.3, 21.6, 20.8 , 13.9. The enantiomeric excess was determined by HPLC with an IA- column. ( $n$-hexane: $i-\operatorname{PrOH}=$ 90:10), $1 \mathrm{~mL} / \mathrm{min}$; major enantiomer $t_{R}=7.99 \mathrm{~min}$, minor enantiomer $t_{R}=15.72 \mathrm{~min}$. HRMS (ESI): $[\mathrm{M}+\mathrm{H}]^{+}$calcd for $\left[\mathrm{C}_{32} \mathrm{H}_{28} \mathrm{~N}_{3} \mathrm{O}_{6} \mathrm{~S}_{2}\right]: 614.1414$, found: 614.1413.

\section{Ethyl}

(4R,6R)-6-acetoxy-4'-oxo-1,3'-diphenyl-3-(thiophen-2-yl)-2'-thioxo-5,6-dihydro-1H-spiro[pyr ano[2,3-c]pyrazole-4,5'-thiazolidine]-6-carboxylate

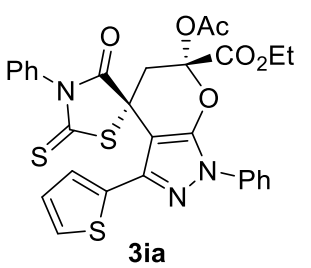

An amorphous solid, $83 \%$ yield $(50 \mathrm{mg}) \cdot[\alpha]_{\mathrm{D}}^{20}=-58\left(c 1.0, \mathrm{CH}_{2} \mathrm{Cl}_{2}, 94 \%\right.$ ee); ${ }^{1} \mathrm{H}$ NMR (400 MHz, $\left.\mathrm{CDCl}_{3}\right): \delta=7.83(\mathrm{~d}, J=8 \mathrm{~Hz}, 2 \mathrm{H})$, 7.41-7.49(m, 6H), 7.29-7.35(m, 2H), 7.13-7.15(m, 1H), 6.51-6.98(br, $2 \mathrm{H}), 4.38(\mathrm{q}, J=7.2 \mathrm{~Hz}, 2 \mathrm{H}), 3.12(\mathrm{~s}, 2 \mathrm{H}), 2.27(\mathrm{~s}, 3 \mathrm{H}), 1.36(\mathrm{t}, J=7.2 \mathrm{~Hz}$, $3 \mathrm{H}) ;{ }^{13} \mathrm{C}$ NMR $\left(150 \mathrm{MHz}, \mathrm{CDCl}_{3}\right): \delta=199.0,174.8,167.7,164.3,147.6$, $142.4,137.3,134.8,133.5,129.7,129.4,129.2$, 128.0, 127.8, 127.5, 127.2 , 127.0, 120.8, 96.9, 95.0, 63.4, 53.0, 41.6, 20.8, 13.9. The enantiomeric excess was determined by HPLC with an IA- column. ( $\mathrm{n}$-hexane: $i-\mathrm{PrOH}=90: 10), 1 \mathrm{~mL} / \mathrm{min}$; major enantiomer $\mathrm{t}_{\mathrm{R}}=10.46 \mathrm{~min}$, minor enantiomer $\mathrm{t}_{\mathrm{R}}=21.27 \mathrm{~min}$. HRMS (ESI): $[\mathrm{M}+\mathrm{H}]^{+}$calcd for $\left[\mathrm{C}_{29} \mathrm{H}_{24} \mathrm{~N}_{3} \mathrm{O}_{6} \mathrm{~S}_{3}\right]: 606.0822$, found: 606.0823 .

\section{Ethyl}

(4R,6R)-6-acetoxy-3-(3,4-dimethoxyphenyl)-4'-oxo-1,3'-diphenyl-2'-thioxo-5,6-dihydro-1H-s piro[pyrano[2,3-c]pyrazole-4,5'-thiazolidine]-6-carboxylate

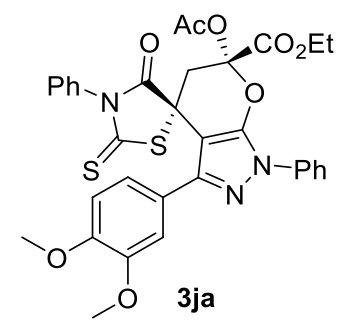

An amorphous solid, $83 \%$ yield $(55 \mathrm{mg})$. m. p.: $176-180^{\circ} \mathrm{C} \cdot[\alpha]_{\mathrm{D}}^{20}=$ 157 (c 1.0, $\mathrm{CH}_{2} \mathrm{Cl}_{2}, 90 \%$ ee); ${ }^{1} \mathrm{H}$ NMR $\left(400 \mathrm{MHz}, \mathrm{CDCl}_{3}\right): \delta=$ 7.84-7.86(m, 2H),7.44-7.48(m, 2H), 7.33-7.41(m, 3H), 7.27-7.32(m, 
1H), 7.17-7.22(m, 2H), 6.86-6.94(m, 1H), 6.31-6.68(br, 2H), 4.37(q, J = 7.2 Hz, 2H), 3.88(s, $3 \mathrm{H}), 3.91(\mathrm{~s}, 3 \mathrm{H}), 3.05(\mathrm{~s}, 2 \mathrm{H}), 2.28(\mathrm{~s}, 3 \mathrm{H}), 1.36(\mathrm{t}, J=7.2 \mathrm{~Hz}, 3 \mathrm{H}) ;{ }^{13} \mathrm{C}$ NMR (100 MHz, $\left.\mathrm{CDCl}_{3}\right): \delta=199.1,174.9,167.7,164.4,150.0,149.4,148.6,147.3,137.5,134.6,129.7,129.3$, 129.2, 128.0, 126.8 ,125.2, 121.0, 120.7, 111.5, 96.9, 94.9, 63.4, 56.1, 56.0, 53.2, 41.4, 20.9, 13.9 . The enantiomeric excess was determined by HPLC with an IA- column. ( $n$-hexane: $i$-PrOH $=$ 90:10), $1 \mathrm{~mL} / \mathrm{min}$; major enantiomer $t_{R}=12.15 \mathrm{~min}$, minor enantiomer $t_{R}=23.12 \mathrm{~min}$. HRMS (ESI): $[\mathrm{M}+\mathrm{H}]^{+}$calcd for $\left[\mathrm{C}_{33} \mathrm{H}_{30} \mathrm{~N}_{3} \mathrm{O}_{8} \mathrm{~S}_{2}\right]: 659.1969$, found: 659.1960 .

\section{Ethyl}

(4R,6R)-6-acetoxy-3-benzyl-4'-oxo-1,3'-diphenyl-2'-thioxo-5,6-dihydro-1H-spiro[pyrano[2,3c]pyrazole-4,5'-thiazolidine]-6-carboxylate

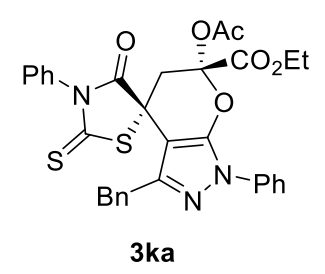

An amorphous solid, $60 \%$ yield $(37 \mathrm{mg}) \cdot[\alpha]_{\mathrm{D}}{ }^{20}=15\left(c 1.0, \mathrm{CH}_{2} \mathrm{Cl}_{2}, 93 \%\right.$ ee); ${ }^{1} \mathrm{H}$ NMR (400 MHz, $\left.\mathrm{CDCl}_{3}\right): \delta=7.73-7.80(\mathrm{~m}, 2 \mathrm{H}), 7.48-7.53(\mathrm{~m}$, $3 \mathrm{H}), 7.40-7.46(\mathrm{~m}, 2 \mathrm{H}), 7.21-7.30(6 \mathrm{H}, \mathrm{m}), 7.03-7.12(\mathrm{~m}, 2 \mathrm{H}), 4.37(\mathrm{q}, J=$ $7.2 \mathrm{~Hz}, 2 \mathrm{H}), 4.05(\mathrm{~d}, J=16.0 \mathrm{~Hz}, 1 \mathrm{H}), 3.85(\mathrm{~d}, J=16.0 \mathrm{~Hz}, 1 \mathrm{H}), 3.16(\mathrm{~d}, J$ $=14.4 \mathrm{~Hz}, 1 \mathrm{H}), 3.06(\mathrm{~d}, J=14.4 \mathrm{~Hz}, 1 \mathrm{H}), 2.17(\mathrm{~s}, 3 \mathrm{H}), 1.35(\mathrm{t}, J=7.2 \mathrm{~Hz}$, $3 \mathrm{H}) ;{ }^{13} \mathrm{C} \mathrm{NMR}\left(100 \mathrm{MHz}, \mathrm{CDCl}_{3}\right): \delta=198.9,175.2,167.8,164.4,147.2,146.9,137.6,136.7$, $134.8,129.8,129.6,129.1,128.7,128.5,128.1,126.7,126.6,120.5,96.9,94.6,63.4,53.2$, 41.2, 33.9, 20.8, 13.9. The enantiomeric excess was determined by HPLC with an IF- column. (n-hexane:i-PrOH = 90:10), $1 \mathrm{~mL} / \mathrm{min}$; major enantiomer $\mathrm{t}_{\mathrm{R}}=15.81 \mathrm{~min}$, minor enantiomer $\mathrm{t}_{\mathrm{R}}$ $=16.67$ min. HRMS (ESI): $[\mathrm{M}+\mathrm{H}]^{+}$calcd for $\left[\mathrm{C}_{32} \mathrm{H}_{28} \mathrm{~N}_{3} \mathrm{O}_{6} \mathrm{~S}_{2}\right]: 614.1414$, found: 614.1413.

\section{Ethyl}

(4R,6R)-6-acetoxy-1-(3-fluorophenyl)-4'-oxo-3,3'-diphenyl-2'-thioxo-5,6-dihydro-1H-spiro[p yrano[2,3-c]pyrazole-4,5'-thiazolidine]-6-carboxylate

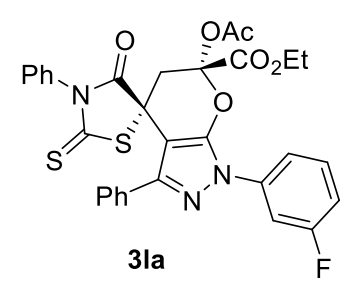

An amorphous solid, $82 \%$ yield $(51 \mathrm{mg})$. m. p.: $176-180^{\circ} \mathrm{C} .[\alpha]_{\mathrm{D}}{ }^{20}=18$ (c $1.0, \mathrm{CH}_{2} \mathrm{Cl}_{2}, 91 \%$ ee); ${ }^{1} \mathrm{H}$ NMR $\left(400 \mathrm{MHz}, \mathrm{CDCl}_{3}\right): \delta=$ 7.63-7.72(m, $4 \mathrm{H}), 7.44-7.50(\mathrm{~m}, 3 \mathrm{H}), 7.38-7.42(\mathrm{~m}, 1 \mathrm{H}), 7.30-7.37(\mathrm{~m}$, $3 \mathrm{H}), 6.96-7.03(\mathrm{~m}, 1 \mathrm{H}), 6.02-6.03(\mathrm{br}, 2 \mathrm{H}), 4.39(\mathrm{q}, J=7.2 \mathrm{~Hz}, 2 \mathrm{H})$, $3.09(\mathrm{~s}, 2 \mathrm{H}), 2.28(\mathrm{~s}, 3 \mathrm{H}), 1.38(\mathrm{t}, J=7.2 \mathrm{~Hz}, 3 \mathrm{H}) ;{ }^{13} \mathrm{C} \mathrm{NMR}(100 \mathrm{MHz}$, $\left.\mathrm{CDCl}_{3}\right): \delta=199.0,174.7,167.6,164.3,162.9(\mathrm{~d}, J=244 \mathrm{~Hz}), 149.0,147.6,138.8(\mathrm{~d}, J=10$ $\mathrm{Hz}), 134.5,132.5,130.5(\mathrm{~d}, \mathrm{~J}=9 \mathrm{~Hz}), 129.6,129.2,129.1,128.2,127.9,115.5(\mathrm{~d}, \mathrm{~J}=3 \mathrm{~Hz})$ 113.6(d, $J=21 \mathrm{~Hz}), 108.3,108.0,96.8,95.6,63.5,52.9$, 41.2, 20.8, 13.9. The enantiomeric excess was determined by HPLC with an IA- column. ( $n$-hexane: $i$-PrOH $=90: 10$ ), $1 \mathrm{~mL} / \mathrm{min}$; major enantiomer $t_{R}=7.99 \mathrm{~min}$, minor enantiomer $t_{R}=16.49 \min$. HRMS $(E S I):[M+H]^{+}$ calcd for $\left[\mathrm{C}_{31} \mathrm{H}_{25} \mathrm{FN}_{3} \mathrm{O}_{6} \mathrm{~S}_{2}\right]$ : 618.1163, found: 618.1160. 


\section{Ethyl}

(4R,6R)-6-acetoxy-1-(3-chlorophenyl)-4'-oxo-3,3'-diphenyl-2'-thioxo-5,6-dihydro-1H-spiro[p yrano[2,3-c]pyrazole-4,5'-thiazolidine]-6-carboxylate

An amorphous solid, $82 \%$ yield $(52 \mathrm{mg}) \cdot[\alpha]_{\mathrm{D}}{ }^{20}=11$ (c 1.0, $\mathrm{CH}_{2} \mathrm{Cl}_{2}, 92 \%$ ee); ${ }^{1} \mathrm{H}$ NMR (400 $\left.\mathrm{MHz}, \quad \mathrm{CDCl}_{3}\right): \delta=7.93-7.97(\mathrm{~m}, \quad 1 \mathrm{H}), \quad 7.76-7.80(\mathrm{~m}, \quad 1 \mathrm{H})$,<smiles>CCOC(C)=O</smiles>
7.65-7.69(m, 2H), 7.44-7.50(m, 3H), 7.31-7.42(m, 4H), 7.27-7.30(m, 1H), 5.72-6.87(br, 2H), 4.45-4.34(m, 2H), 3.09(s, 2H), $2.28(\mathrm{~s}, 3 \mathrm{H}), 1.38(\mathrm{t}, J=7.2 \mathrm{~Hz}, 3 \mathrm{H}) ;{ }^{13} \mathrm{C} \mathrm{NMR}\left(100 \mathrm{MHz}, \mathrm{CDCl}_{3}\right)$ : $\delta=199.0,174.7,164.3,149.1,147.5,138.4,135.0,134.5,132.4$, Cl $130.3,129.6,129.2,129.1,128.6,127.9,126.8,120.8,118.3,96.8$, 95.6, 63.5, 52.9, 41.2, 20.8, 13.9. The enantiomeric excess was determined by HPLC with an IA- column. (n-hexane: $i-\mathrm{PrOH}=90: 10), 1 \mathrm{~mL} / \mathrm{min}$; major enantiomer $\mathrm{t}_{\mathrm{R}}=7.86 \mathrm{~min}$, minor enantiomer $t_{R}=16.27$ min. HRMS (ESI): $[\mathrm{M}+\mathrm{H}]^{+}$calcd for $\left[\mathrm{C}_{31} \mathrm{H}_{25} \mathrm{ClN}_{3} \mathrm{O}_{6} \mathrm{~S}_{2}\right]: 634.0868$, found: 634.0864 .

\section{Ethyl}

(4R,6R)-6-acetoxy-1-(3-bromophenyl)-4'-oxo-3,3'-diphenyl-2'-thioxo-5,6-dihydro-1H-spiro[p yrano[2,3-c]pyrazole-4,5'-thiazolidine]-6-carboxylate

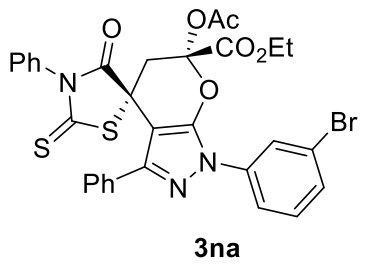

An amorphous solid, $81 \%$ yield $(55 \mathrm{mg}) .[\alpha]_{\mathrm{D}}^{20}=9\left(c 1.0, \mathrm{CH}_{2} \mathrm{Cl}_{2}\right.$, $91 \%$ ee); ${ }^{1} \mathrm{H}$ NMR $\left(600 \mathrm{MHz}, \mathrm{CDCl}_{3}\right): \delta=8.09-8.11(\mathrm{~m}, 1 \mathrm{H})$, 7.80-7.84(m, 1H), 7.65-7.69(m, 2H), 7.44-7.79(m, 3H), 7.41-7.44(m, $1 \mathrm{H}), 7.36-7.40(\mathrm{~m}, 1 \mathrm{H}), 7.31-7.36(\mathrm{~m}, 3 \mathrm{H}), 5,54-6.89(\mathrm{br}, 2 \mathrm{H}), 4.39(\mathrm{~m}$, $2 \mathrm{H}), 3.09(\mathrm{~s}, 2 \mathrm{H}), 2.28(\mathrm{~s}, 3 \mathrm{H}), 1.39(\mathrm{t}, J=7.2 \mathrm{~Hz}, 3 \mathrm{H}) ;{ }^{13} \mathrm{C} \mathrm{NMR}$ $\left(150 \mathrm{MHz}, \mathrm{CDCl}_{3}\right): \delta=199.0,174,7,167.6,164.3,149.2,147.6,138.6,134.5,132.5,130.5$, 129.7, 129.6, 129.2, 128.6, 127.9, 123.7, 122.8, 118.8, 96.3, 95.6, 63.5, 52.9, 41.3, 20.8, 13.9. The enantiomeric excess was determined by HPLC with an IA- column. $(n$-hexane: $i-\mathrm{PrOH}=$ 90:10), $1 \mathrm{~mL} / \mathrm{min}$; major enantiomer $t_{R}=8.12 \mathrm{~min}$, minor enantiomer $t_{R}=16.64 \mathrm{~min}$. HRMS (ESI): $[\mathrm{M}+\mathrm{H}]^{+}$calcd for $\left[\mathrm{C}_{31} \mathrm{H}_{25} \mathrm{BrN}_{3} \mathrm{O}_{6} \mathrm{~S}_{2}\right]: 678.0363,680.0342$, found: $678.0360,680.0337$

\section{Ethyl}

(4R,6R)-6-acetoxy-4'-oxo-3,3'-diphenyl-2'-thioxo-1-(m-tolyl)-5,6-dihydro-1H-spiro[pyrano[2, 3-c]pyrazole-4,5'-thiazolidine]-6-carboxylate

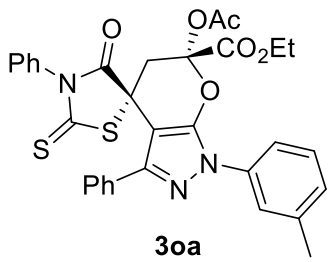

An amorphous solid, $81 \%$ yield $(49 \mathrm{mg}) \cdot[\alpha]_{\mathrm{D}}^{20}=10\left(c 1.0, \mathrm{CH}_{2} \mathrm{Cl}_{2}, 95 \%\right.$ ee); ${ }^{1} \mathrm{H}$ NMR $\left(600 \mathrm{MHz}, \mathrm{CDCl}_{3}\right): \delta=7.70-7.73(\mathrm{~m}, 2 \mathrm{H}), 7.66-7.69(\mathrm{~m}$, $2 \mathrm{H}), \quad 7.45-7.47(\mathrm{~m}, \quad 2 \mathrm{H}), \quad 7.43-7.44(\mathrm{~m}, \quad 1 \mathrm{H}), \quad 7.31-7.39(\mathrm{~m}, \quad 3 \mathrm{H})$, 
7.25-7.27(m, 1H), 7.24- 7.25(m, 1H), 5.79-6.88(br, $2 \mathrm{H}), 4.37(\mathrm{q}, J=7.2 \mathrm{~Hz}, 2 \mathrm{H}), 3.11(\mathrm{~d}, J=$ $14.4 \mathrm{~Hz}, 1 \mathrm{H}), 3.07(\mathrm{~d}, J=14.4 \mathrm{~Hz}, 1 \mathrm{H}), 2.38(\mathrm{~s}, 3 \mathrm{H}), 2.27(\mathrm{~s}, 3 \mathrm{H}), 1.36(\mathrm{t}, J=7.2 \mathrm{~Hz}, 3 \mathrm{H}) ;{ }^{13} \mathrm{C}$ NMR (150 MHz, $\left.\mathrm{CDCl}_{3}\right): \delta=199.2,174.9,167.7,164.4,148.4,147.1,136.7,135.1,134.6$, $132.8,129.7,129.5,129.2,129.1,128.9,128.6,127.9,120.7,96.9,95.0,63.4,55.0,53.2,41.2$, 20.8, 13.9. The enantiomeric excess was determined by HPLC with an IA- column. ( $n$-hexane: $i$-PrOH $=90: 10), 1 \mathrm{~mL} / \mathrm{min}$; major enantiomer $\mathrm{t}_{\mathrm{R}}=8.20 \mathrm{~min}$, minor enantiomer $\mathrm{t}_{\mathrm{R}}$ $=16.69 \mathrm{~min}$. HRMS (ESI): $[\mathrm{M}+\mathrm{H}]^{+}$calcd for $\left[\mathrm{C}_{32} \mathrm{H}_{28} \mathrm{~N}_{3} \mathrm{O}_{6} \mathrm{~S}_{2}\right]: 614.1414$, found: 614.1413.

\section{Ethyl}

(4R,6R)-6-acetoxy-1-(3-methoxyphenyl)-4'-oxo-3,3'-diphenyl-2'-thioxo-5,6-dihydro-1H-spiro [pyrano[2,3-c]pyrazole-4,5'-thiazolidine]-6-carboxylate

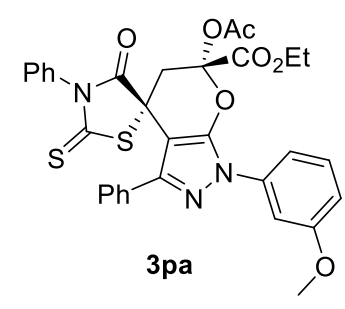

An amorphous solid, $81 \%$ yield $(52 \mathrm{mg}) \cdot[\alpha]_{\mathrm{D}}{ }^{20}=26\left(c 1.0, \mathrm{CH}_{2} \mathrm{Cl}_{2}, 90 \%\right.$ ee); ${ }^{1} \mathrm{H}$ NMR (400 MHz, $\left.\mathrm{CDCl}_{3}\right): \delta=7.64-7.72(\mathrm{~m}, 2 \mathrm{H}), 7.43-7.50(\mathrm{~m}$, $5 \mathrm{H}), 7.29-7.40(\mathrm{~m}, 4 \mathrm{H}), 6.79-6.90(\mathrm{~m}, 1 \mathrm{H}), 5.96-6.69(\mathrm{br}, 2 \mathrm{H}), 4.37(\mathrm{q}, J$ $=7.2 \mathrm{~Hz}, 2 \mathrm{H}), 3.86(\mathrm{~s}, 3 \mathrm{H}), 3.10(\mathrm{~d}, J=14.4 \mathrm{~Hz}, 1 \mathrm{H}), 3.06(\mathrm{~d}, J=14.4$ $\mathrm{Hz}, 1 \mathrm{H}), 2.27(\mathrm{~s}, 3 \mathrm{H}), 1.35(\mathrm{t}, J=7.2 \mathrm{~Hz}, 3 \mathrm{H}) ;{ }^{13} \mathrm{C} \mathrm{NMR}(100 \mathrm{MHz}$,

$\left.\mathrm{CDCl}_{3}\right): \delta=199.2,174.8,167.7,164.4,160.1,148.5,147.3,138.5,134.5,132.7,129.9,129.5$, 129.2, 129.1, 129.0, 128.6, 127.9, 113.5, 112.7, 105.6, 96.7, 95.2, 63.4, 55.4, 53.1, 41.2, 20.8, 13.9. The enantiomeric excess was determined by HPLC with an IA- column. (n-hexane: $i-\mathrm{PrOH}=90: 10), 1 \mathrm{~mL} / \mathrm{min}$; major enantiomer $\mathrm{t}_{\mathrm{R}}=8.8 \mathrm{~min}$, minor enantiomer $\mathrm{t}_{\mathrm{R}}=$ 16.18 min. HRMS (ESI): $[\mathrm{M}+\mathrm{H}]^{+}$calcd for $\left[\mathrm{C}_{32} \mathrm{H}_{28} \mathrm{~N}_{3} \mathrm{O}_{4} \mathrm{~S}_{2}\right]$ : 630.1363 , found: 630.1363 .

\section{Ethyl}

(4R,6R)-6-acetoxy-1-(4-bromophenyl)-4'-oxo-3,3'-diphenyl-2'-thioxo-5,6-dihydro-1H-spiro[p yrano[2,3-c]pyrazole-4,5'-thiazolidine]-6-carboxylate

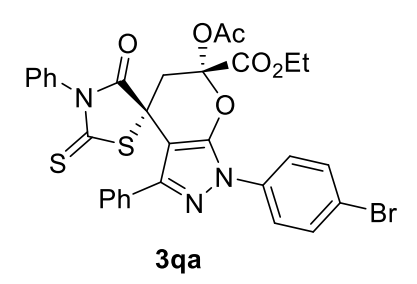

An amorphous solid, $87 \%$ yield $(58 \mathrm{mg}) .[\alpha]_{\mathrm{D}}{ }^{20}=42\left(c 1.0, \mathrm{CH}_{2} \mathrm{Cl}_{2}\right.$, $91 \%$ ee); ${ }^{1} \mathrm{H}$ NMR $\left(400 \mathrm{MHz}, \mathrm{CDCl}_{3}\right): \delta=7.77(\mathrm{~d}, J=9.2 \mathrm{~Hz}, 2 \mathrm{H})$, $7.64-7.69(\mathrm{~m}, \quad 2 \mathrm{H}), \quad 7.56-7.61(\mathrm{~m}, \quad 2 \mathrm{H}), \quad 7.43-7.49(\mathrm{~m}, \quad 3 \mathrm{H})$, $7.30-7.40(\mathrm{~m}, 3 \mathrm{H}), 5.87-6.77(\mathrm{br}, 2 \mathrm{H}), 4.38(\mathrm{q}, J=7.2 \mathrm{~Hz}, 2 \mathrm{H})$, $3.08(\mathrm{~s}, 2 \mathrm{H}), 2.28(\mathrm{~s}, 3 \mathrm{H}), 1.36(\mathrm{t}, J=7.2 \mathrm{~Hz}, 3 \mathrm{H}) ;{ }^{13} \mathrm{C} \mathrm{NMR}(100$ $\left.\mathrm{MHz}, \mathrm{CDCl}_{3}\right): \delta=199.0,174.7,167.7,164.3,149.0,147.4,136.5,134.5,132.5,132.3,129.6$, $129.3,129.2$, 129.1, 128.5, 127.9, 122.0, 120.2, 96.8, 95.5, 63.5, 52.9, 41.2, 20.8, 13.9. The enantiomeric excess was determined by HPLC with an IA- column. (n-hexane: $i-\operatorname{PrOH}=$ 90:10), $1 \mathrm{~mL} / \mathrm{min}$; major enantiomer $t_{R}=9.08 \mathrm{~min}$, minor enantiomer $t_{R}=18.62 \mathrm{~min}$. HRMS (ESI): $[\mathrm{M}+\mathrm{H}]^{+}$calcd for $\left[\mathrm{C}_{31} \mathrm{H}_{25} \mathrm{BrN}_{3} \mathrm{O}_{6} \mathrm{~S}_{2}\right]$ : 678.0363, 680.0342, found: 678.0361, 680.0342. 


\section{Ethyl}

(4R,6R)-6-acetoxy-4'-oxo-3,3'-diphenyl-2'-thioxo-1-(p-tolyl)-5,6-dihydro-1H-spiro[pyrano[2, 3-c]pyrazole-4,5'-thiazolidine]-6-carboxylate

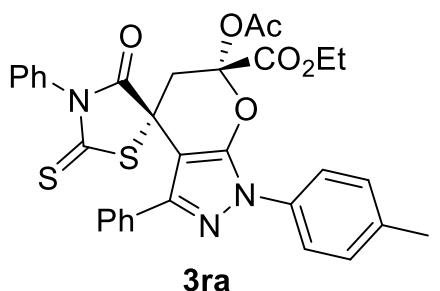

An amorphous solid, $81 \%$ yield $(50 \mathrm{mg}) \cdot[\alpha]_{\mathrm{D}}{ }^{20}=-31$ (c 1.0, $\mathrm{CH}_{2} \mathrm{Cl}_{2}, 93 \%$ ee); ${ }^{1} \mathrm{H}$ NMR (400 $\left.\mathrm{MHz}, \mathrm{CDCl}_{3}\right): \delta=$ 7.74-7.65(m, 4H), 7.43-7.48(m, 3H), 7.31-7.40(m, 3H), 7.22-7.29(m, 2H), 5.71-6.92(br, 2H), 4.37(q, $J=7.2 \mathrm{~Hz}, 2 \mathrm{H})$, $3.11(\mathrm{~d}, J=14.4 \mathrm{~Hz}, 1 \mathrm{H}), 3.07(\mathrm{~d}, J=14.4 \mathrm{~Hz}, 1 \mathrm{H}), 2.38(\mathrm{~s}, 3 \mathrm{H})$, 2.27(s, 3H), 1.36(t, $J=7.2 \mathrm{~Hz}, 3 \mathrm{H}) ;{ }^{13} \mathrm{C}$ NMR $\left(100 \mathrm{MHz}, \mathrm{CDCl}_{3}\right): \delta=199.2,174.9,167.7$, $164.4,148.4$, 147.1, 136.7, 135.0, 134.5, 132.8, 129.7, 129.5, 129.2, 129.1, 128.9, 128.6, $127.9,120.7,96.8,95.0,63.4,53.1,41.2,21.0,20.8,13.9$. The enantiomeric excess was determined by HPLC with an IA- column. (n-hexane: $i$ PrOH $=90: 10$ ), $1 \mathrm{~mL} / \mathrm{min}$; major enantiomer $t_{R}=8.11 \mathrm{~min}$, minor enantiomer $t_{R}=16.15$ min. HRMS (ESI): $[\mathrm{M}+\mathrm{H}]^{+}$calcd for $\left[\mathrm{C}_{32} \mathrm{H}_{28} \mathrm{~N}_{3} \mathrm{O}_{6} \mathrm{~S}_{2}\right]$ : 614.1414, found: 614.1414.

\section{Ethyl}

(4R,6R)-6-acetoxy-1-(3-chloro-4-methylphenyl)-4'-oxo-3,3'-diphenyl-2'-thioxo-5,6-dihydro-1

\section{H-spiro[pyrano[2,3-c]pyrazole-4,5'-thiazolidine]-6-carboxylate}

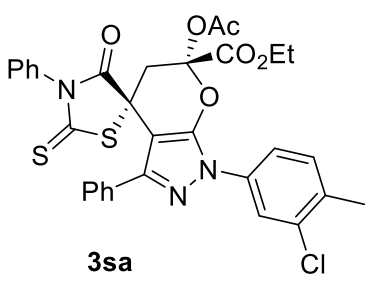

An amorphous solid, $84 \%$ yield $(54 \mathrm{mg}) \cdot[\alpha]_{\mathrm{D}}^{20}=-11\left(c 1.0, \mathrm{CH}_{2} \mathrm{Cl}_{2}\right.$, $92 \%$ ee); ${ }^{1} \mathrm{H}$ NMR $\left(600 \mathrm{MHz}, \mathrm{CDCl}_{3}\right): \delta=7.89-7.93(\mathrm{~m}, 1 \mathrm{H})$, 7.64-7.71(m, 3H), 7.44-7.50(m, 3H), 7.29-7.40(m, 4H), 6.04-7.11(br, 2H), 4.33-4.44(m, 2H), 3.09(s, 2H), 2.39(s, 3H), $2.27(\mathrm{~s}, 3 \mathrm{H}), 1.38(\mathrm{t}, J=7.2 \mathrm{~Hz}, 3 \mathrm{H}) ;{ }^{13} \mathrm{C}$ NMR $\left(100 \mathrm{MHz}, \mathrm{CDCl}_{3}\right)$ : $\delta=199.0,174.7,167.6,164.3,148.9,147.4,136.2$, 134.8, 134.6, 132.6, 131.3, 131.3, 129.6, 129.2, 129.1, 128.6, 127.9, 121.4, 121.3, 118.6, 118.5, 96.9, 95.4, 63.4, 53.0, 41.2, 20.8, 19.6, 13.9. The enantiomeric excess was determined by HPLC with an IA- column. (n-hexane:i-PrOH $=90: 10$ ), $1 \mathrm{~mL} / \mathrm{min}$; major enantiomer $\mathrm{t}_{\mathrm{R}}=8.06 \mathrm{~min}$, minor enantiomer $\mathrm{t}_{\mathrm{R}}$ $=15.98$ min. HRMS (ESI): $[\mathrm{M}+\mathrm{H}]^{+}$calcd for $\left[\mathrm{C}_{32} \mathrm{H}_{27} \mathrm{ClN}_{3} \mathrm{O}_{6} \mathrm{~S}_{2}\right]: 648.1024$, found: 648.1024.

\section{Ethyl}

(4R,6R)-6-acetoxy-2',4'-dioxo-1,3,3'-triphenyl-5,6-dihydro-1H-spiro[pyrano[2,3-c]pyrazole-4 ,5'-thiazolidine]-6-carboxylate

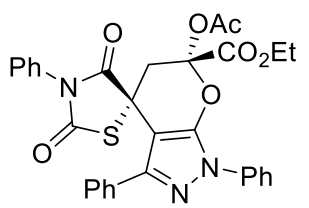

White solid, $68 \%$ yield $(39 \mathrm{mg})$. m. p.: $165-167^{\circ} \mathrm{C}[\alpha]_{\mathrm{D}}{ }^{20}=-14(c 1.0$, $\mathrm{CH}_{2} \mathrm{Cl}_{2}, 92 \%$ ee); ${ }^{1} \mathrm{H}$ NMR (400 MHz, $\left.\mathrm{CDCl}_{3}\right): \delta=7.83-7.88(\mathrm{~m}, 2 \mathrm{H})$, 
7.68-7.73(m, 2H), 7.43-7.50(m, 5H), 7.28-7.37(m, 4H), 6.46-6.51(m, 2H), 4.38(q, J = 7.2 Hz, $2 \mathrm{H}), 3.18(\mathrm{~d}, J=14.4 \mathrm{~Hz}, 1 \mathrm{H}), 3.06(\mathrm{~d}, J=14.4 \mathrm{~Hz}, 1 \mathrm{H}), 2.26(\mathrm{~s}, 3 \mathrm{H}), 1.36(\mathrm{t}, J=7.2 \mathrm{~Hz}, 3 \mathrm{H})$; ${ }^{13} \mathrm{C}$ NMR $\left(100 \mathrm{MHz}, \mathrm{CDCl}_{3}\right): \delta=173.1,169.7,167.8,164.4,148.6,147.3,137.4,132.7$, 132.2, 129.3, 129.2, 129.2, 129.1, 129.0, 128.7, 126.9, 120.7, 97.0, 95.6, 63.4, 51.5, 41.3, 20.8, 13.9. HRMS (ESI): $[\mathrm{M}+\mathrm{H}]^{+}$calcd for $\left[\mathrm{C}_{31} \mathrm{H}_{26} \mathrm{~N}_{3} \mathrm{O}_{7} \mathrm{~S}\right]: 584.1421$, found: 584.1423 .

Ethyl (E)-2-oxo-3-(4-oxo-3-phenyl-2-thioxothiazolidin-5-ylidene)propanoate

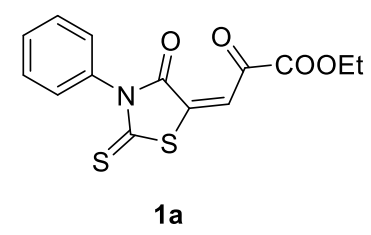

red solid, $36 \%$ yield; m. p.: $162-166^{\circ} \mathrm{C} .{ }^{1} \mathrm{H}$ NMR $\left(400 \mathrm{MHz}, \mathrm{CDCl}_{3}\right)$ : $\delta=7.90-7.95(\mathrm{~s}, 1 \mathrm{H}), 7.52-7.59(\mathrm{~m}, 3 \mathrm{H}), 7.23-7.28(\mathrm{~m}, 2 \mathrm{H}), 4.43(\mathrm{q}, J$ $=7.2 \mathrm{~Hz}, 2 \mathrm{H}), 1.43(\mathrm{t}, J=7.2 \mathrm{~Hz}, 3 \mathrm{H}) ;{ }^{13} \mathrm{C} \mathrm{NMR}\left(100 \mathrm{MHz}, \mathrm{CDCl}_{3}\right)$ : $\delta=195.6,181.5,166.4,159.6,145.8,133.7,130.0,129.6,116.2$,

63.3, 13.9. HRMS (ESI): $[M+H]^{+}$calcd for $\left[\mathrm{C}_{14} \mathrm{H}_{12} \mathrm{NO}_{4} \mathrm{~S}_{2}\right]$ : 322.0202 , found: 322.0203 .

Ethyl (E)-3-(3-(4-fluorophenyl)-4-oxo-2-thioxothiazolidin-5-ylidene)-2-oxopropanoate

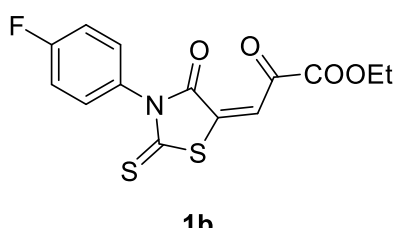

red solid, $29 \%$ yield; m. p.: $173-175^{\circ} \mathrm{C} .{ }^{1} \mathrm{H}$ NMR $(400 \mathrm{MHz}$, $\left.\mathrm{CDCl}_{3}\right): \delta=7.89-7.93(\mathrm{~s}, 1 \mathrm{H}), 7.28-7.24(\mathrm{~m}, 2 \mathrm{H}), 7.23-7.19(\mathrm{~m}$, $2 \mathrm{H}), 4.43(\mathrm{q}, J=7.2 \mathrm{~Hz}, 2 \mathrm{H}), 1.43(\mathrm{t}, J=7.2 \mathrm{~Hz}, 3 \mathrm{H}) ;{ }^{13} \mathrm{C} \mathrm{NMR}$ $\left(100 \mathrm{MHz}, \mathrm{CDCl}_{3}\right): \delta=195.6,181.4,166.3,163.0\left(\mathrm{~d}, J_{C-F}=250\right.$ Hz), 159.6, 145.4, 130.1, 130.0, 129.4, 129.4, 116.9, 116.7, 116.4, 63.3, 13.9. HRMS (ESI): $[\mathrm{M}+\mathrm{H}]^{+}$calcd for $\left[\mathrm{C}_{14} \mathrm{H}_{11} \mathrm{NFO}_{4} \mathrm{~S}_{2}\right]: 340.0108$, found: 340.0110 .

Ethyl (E)-3-(3-(4-chlorophenyl)-4-oxo-2-thioxothiazolidin-5-ylidene)-2-oxopropanoate

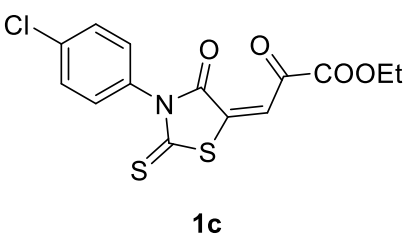

red solid, 30\% yield; m. p.: $159-164^{\circ} \mathrm{C} .{ }^{1} \mathrm{H}$ NMR $(400 \mathrm{MHz}$, $\left.\mathrm{CDCl}_{3}\right): \delta=7.92-7.87(\mathrm{~s}, 1 \mathrm{H}), 7.53-7.57(\mathrm{~m}, 2 \mathrm{H}), 7.20-7.29(\mathrm{~m}$, $2 \mathrm{H}), 4.43(\mathrm{q}, J=6.8 \mathrm{~Hz}, 2 \mathrm{H}), 1.43(\mathrm{t}, J=6.8 \mathrm{~Hz}, 3 \mathrm{H}) ;{ }^{13} \mathrm{C} \mathrm{NMR}$ $\left(100 \mathrm{MHz}, \mathrm{CDCl}_{3}\right): \delta=195.4,181.5,166.2,159.7,145.4,136.2$,

132.1, 130.0, 129.5, 116.5, 63.4, 13.9. HRMS (ESI): $[\mathrm{M}+\mathrm{H}]^{+}$calcd for $\left[\mathrm{C}_{14} \mathrm{H}_{11} \mathrm{NClO}_{4} \mathrm{~S}_{2}\right]$ : 355.9813 , found: 355.9816 .

Ethyl (E)-2-oxo-3-(4-oxo-2-thioxo-3-(p-tolyl)thiazolidin-5-ylidene)propanoate

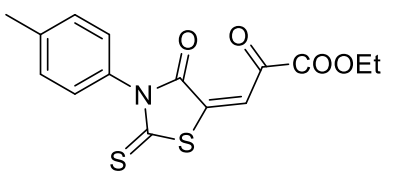

$1 d$ red solid, $31 \%$ yield; m. p.: $177-179^{\circ} \mathrm{C} .{ }^{1} \mathrm{H}$ NMR $(400 \mathrm{MHz}$, $\left.\mathrm{CDCl}_{3}\right): \delta=7.88-7.93(\mathrm{~s}, 1 \mathrm{H}), 7.32-7.39(\mathrm{~m}, 2 \mathrm{H}), 7.10-7.14(\mathrm{~m}, 2 \mathrm{H})$, 
$4.09(\mathrm{q}, J=7.2 \mathrm{~Hz}, 2 \mathrm{H}), 2.42(\mathrm{~s}, 3 \mathrm{H}), 1.41(\mathrm{t}, J=7.2 \mathrm{~Hz}, 3 \mathrm{H}) ;{ }^{13} \mathrm{C} \mathrm{NMR}\left(100 \mathrm{MHz}, \mathrm{CDCl}_{3}\right): \delta$ $=195.7,181.3,166.3,159.5,145.6,140.1,130.9,130.2$, 127.6, 116.1, 63.2, 21.2, 13.8. HRMS (ESI): $[\mathrm{M}+\mathrm{H}]^{+}$calcd for $\left[\mathrm{C}_{15} \mathrm{H}_{14} \mathrm{NO}_{4} \mathrm{~S}_{2}\right]: 336.0359$, found: 336.0359 .

Ethyl (E)-3-(3-(4-methoxyphenyl)-4-oxo-2-thioxothiazolidin-5-ylidene)-2-oxopropanoate

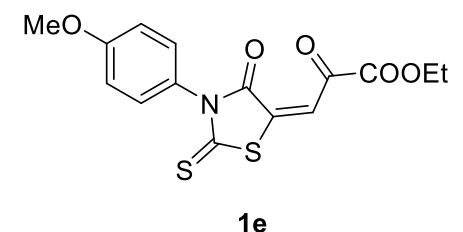

red solid, $35 \%$ yield; m. p.: $183-188^{\circ} \mathrm{C} .{ }^{1} \mathrm{H}$ NMR (400 MHz, $\left.\mathrm{CDCl}_{3}\right): \delta=7.90-7.93(\mathrm{~s}, 1 \mathrm{H}), 7.14-7.19(\mathrm{~m}, 2 \mathrm{H}), 7.02-7.07(\mathrm{~m}$, $2 \mathrm{H}), 4.43(\mathrm{q}, J=7.2 \mathrm{~Hz}, 2 \mathrm{H}), 3.86(\mathrm{~s}, 3 \mathrm{H}), 1.43(\mathrm{t}, J=7.2 \mathrm{~Hz}$, $3 \mathrm{H}) ;{ }^{13} \mathrm{C}$ NMR (100 MHz, $\left.\mathrm{CDCl}_{3}\right): \delta=195.9,181.5,166.5$, 160.4, 159.7, 145.7, 129.2, 125.9, 116.2, 114.8, 114.2, 63.3, 55.4, 13.9. HRMS (ESI): $[\mathrm{M}+\mathrm{H}]^{+}$ calcd for $\left[\mathrm{C}_{15} \mathrm{H}_{14} \mathrm{NO}_{5} \mathrm{~S}_{2}\right]$ : 352.0308 , found: 352.0309 . 


\section{X-ray Crystallographic Data of Compound 3ca}

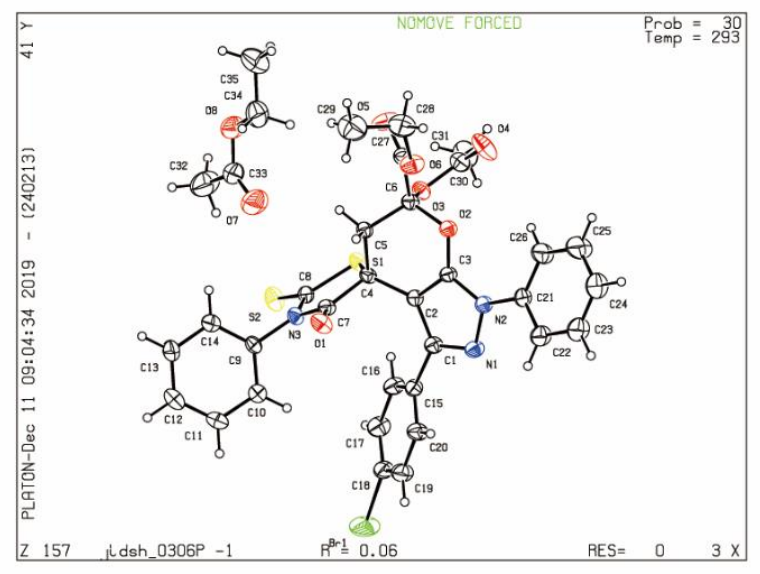<smiles>CCOC(=O)C1(C(=O)OCC)C[C@@]2(SC(=S)N(c3ccccc3)C2=O)c2c(-c3ccc(Br)cc3)nn(-c3ccccc3)c2O1</smiles>

\begin{tabular}{|c|c|c|c|}
\hline \multirow{2}{*}{$\begin{array}{l}\text { Bond precision: } \\
\text { Cell: }\end{array}$} & \multirow{2}{*}{$\begin{array}{l}C-C=0.0057 \mathrm{~A} \\
\mathrm{a}=8.3570(9)\end{array}$} & \multicolumn{2}{|c|}{ Wavelength $=0.71073$} \\
\hline & & $b=10.7681(7)$ & $\mathrm{c}=20.9839(12)$ \\
\hline & Alpha $=88.273$ & Beta $=82.022$ & Gamma $=73.587$ \\
\hline \multirow[t]{2}{*}{ Temperature: } & \multicolumn{3}{|l|}{$293 \mathrm{~K}$} \\
\hline & Calculated & \multicolumn{2}{|c|}{ Reported } \\
\hline Volume & $1793.8(3)$ & \multicolumn{2}{|c|}{$1793.8(3)$} \\
\hline Space group & $\mathrm{P}-1$ & \multicolumn{2}{|c|}{$P-1$} \\
\hline Hall group & $-\mathrm{P} 1$ & \multicolumn{2}{|c|}{$-\mathrm{P} 1$} \\
\hline Moiety formula & $\mathrm{C}_{31} \mathrm{H}_{24} \mathrm{BrN}_{3} \mathrm{O}_{6} \mathrm{~S}_{2}$ & \multicolumn{2}{|c|}{$\mathrm{C}_{31} \mathrm{H}_{24} \mathrm{BrN}_{3} \mathrm{O}_{6} \mathrm{~S}_{2}$} \\
\hline Sum formula & $\mathrm{C}_{35} \mathrm{H}_{32} \mathrm{BrN}_{3} \mathrm{O}_{8} \mathrm{~S}_{2}$ & \multicolumn{2}{|c|}{$\mathrm{C}_{35} \mathrm{H}_{32} \mathrm{BrN}_{3} \mathrm{O}_{8} \mathrm{~S}_{2}$} \\
\hline $\mathrm{Mr}$ & 766.66 & \multicolumn{2}{|c|}{766.66} \\
\hline $\mathrm{Dx}, \mathrm{g} \mathrm{cm}^{-3}$ & 1.419 & \multicolumn{2}{|c|}{1.419} \\
\hline $\mathrm{Z}$ & 2 & \multicolumn{2}{|l|}{2} \\
\hline $\mathrm{Mu}\left(\mathrm{mm}^{-1}\right)$ & 1.316 & \multicolumn{2}{|c|}{1.316} \\
\hline F000 & 788.0 & \multicolumn{2}{|c|}{788.0} \\
\hline F000' & \multicolumn{3}{|l|}{788.26} \\
\hline h, k, lmax & $10,13,25$ & \multicolumn{2}{|c|}{$10,13,25$} \\
\hline Nref & 7072 & \multicolumn{2}{|c|}{7056} \\
\hline Tmin,Tmax & $0.789,0.854$ & \multicolumn{2}{|c|}{$0.463,1.000$} \\
\hline Tmin’ & \multicolumn{3}{|l|}{0.789} \\
\hline $\begin{array}{l}\text { Correction meth } \\
\text { AbsCorr = MUL }\end{array}$ & orted T Limits: Tn & $\operatorname{in}=0.463 \mathrm{Tmax}$ & .000 \\
\hline Data completene & & heta $(\max )=26.0$ & \\
\hline $\mathrm{R}($ reflections $)=($ & & $\mathrm{R} 2($ reflections $)=$ & 309(7056) \\
\hline$S=1.009$ & & par $=446$ & \\
\hline Displacement ell & drawn at $30 \%$ pro & bability level & \\
\hline
\end{tabular}




\section{The Discussion and Determination of Absolute Configuration of}

\section{Compound}

The relative configuration of compound 3ca was acquired through X-ray Crystallographic Data of Racemic Compound 3ca. In order to confirm the absolute configuration (AC) of compounds 3ca, the ECD spectra were calculated by the TD-DFT method, which has been proven to be useful in predicting ECD spectra and assigning the AC of organic molecules (N. Berova, L. D. Bari, G. Pescitelli, Chem. Soc. Rev. 2007, 36, 914-931). Calculations of the ECD spectra of $R R$-3ca and $S S$-3ca (see Figure S1 in Supporting Information) were carried out using the TD-M06/6-31+G** level with Gaussian 09. Electronic excitation energies $(\mathrm{nm})$ and rotational strengths $(\Delta \varepsilon)$ were calculated for 3ca. In order to cover the $220-360 \mathrm{~nm}$ range, 60 transitions were calculated. As shown in Figure $\mathrm{S} 2$, the simulated spectra are in good agreement with the experimental spectral data of $R R-\mathbf{3 c a}$, and the $R R$ configuration could be reliably assigned to compound $\mathbf{3 c a}$.

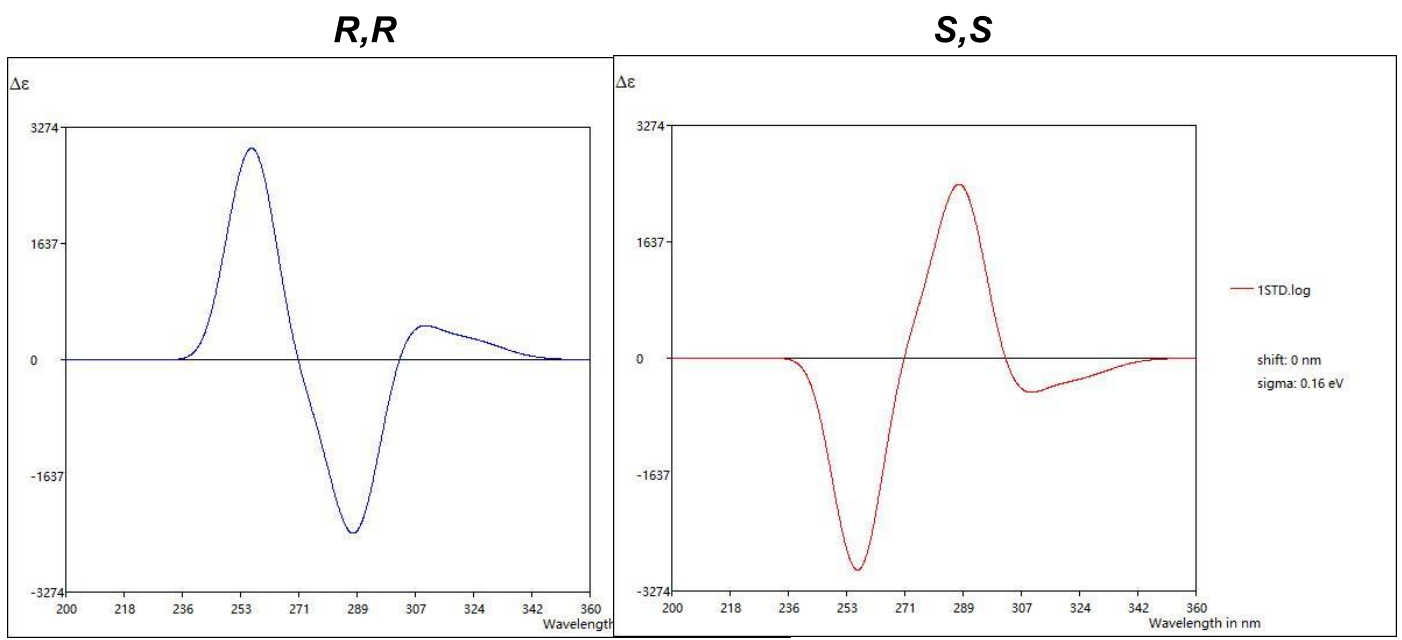

Figure S1. Simulated ECD spectrum through calculations

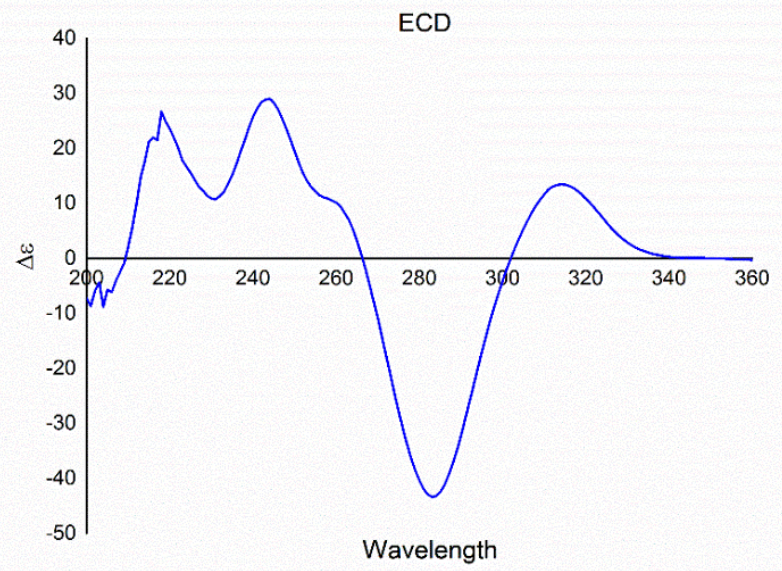

Figure S2. Experimental ECD spectrum (sample concentration: $0.1 \mathrm{mg} / \mathrm{mL}$ in DCM) 


\section{HPLC and NMR Spectrogram}

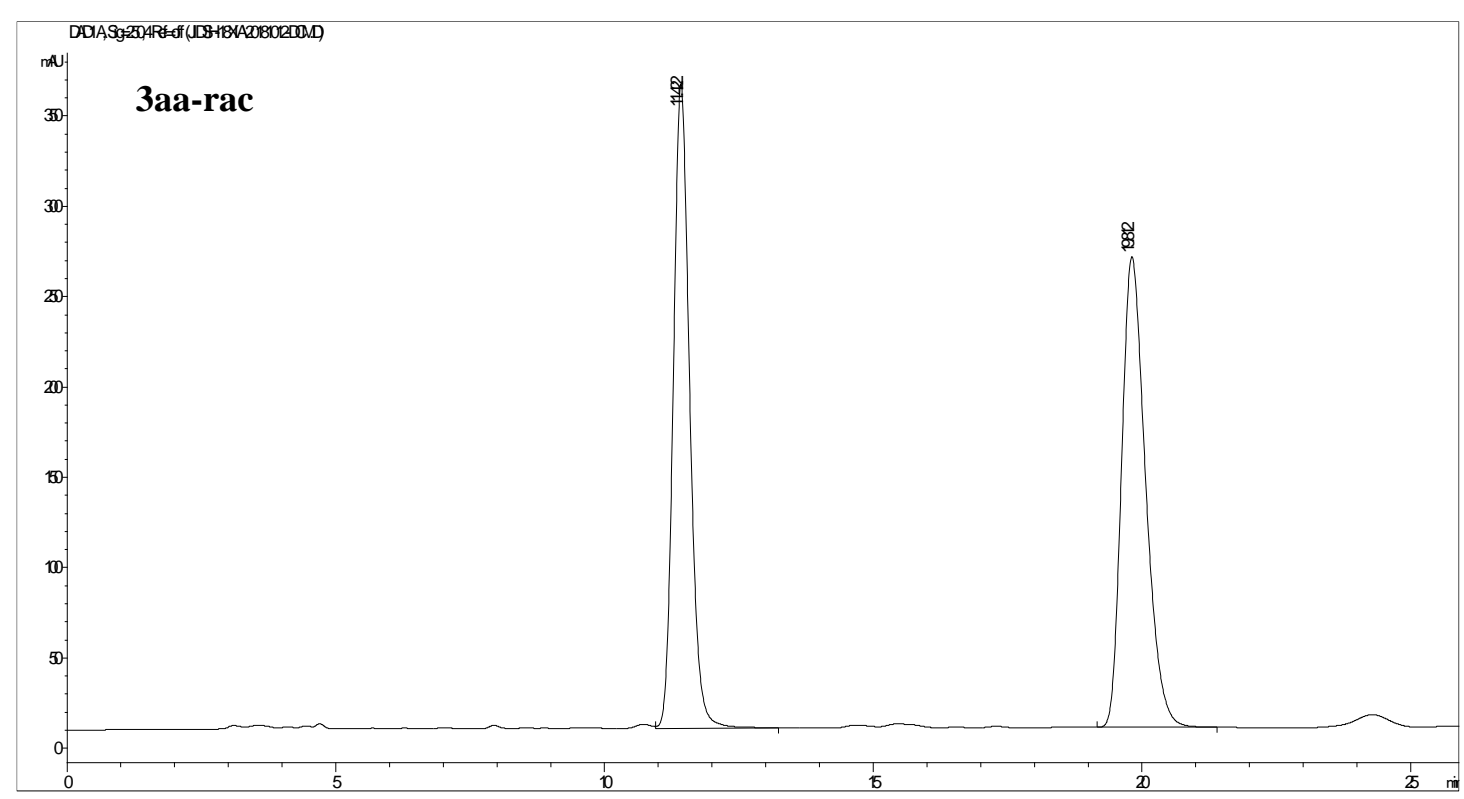

\begin{tabular}{|c|c|c|c|c|c|}
\hline Peak & $\begin{array}{c}\text { Processed } \\
\text { Channel }\end{array}$ & $\begin{array}{c}\text { Retention } \\
\text { Time (min) }\end{array}$ & $\begin{array}{c}\text { Peak Area } \\
\left(\mathrm{mAU}^{*}\right)\end{array}$ & $\begin{array}{c}\text { Peak Height } \\
(\mathrm{mAU})\end{array}$ & $\begin{array}{c}\text { Peak Area } \\
(\%)\end{array}$ \\
\hline 1 & $\begin{array}{c}\text { DAD 254, 4 } \\
\mathrm{nm}\end{array}$ & 11.422 & 7542.5 & 356.6 & 48.405 \\
\hline 2 & $\begin{array}{c}\text { DAD 254, 4 } \\
\mathrm{nm}\end{array}$ & 19.812 & 8039.7 & 260.5 & 51.595 \\
\hline
\end{tabular}

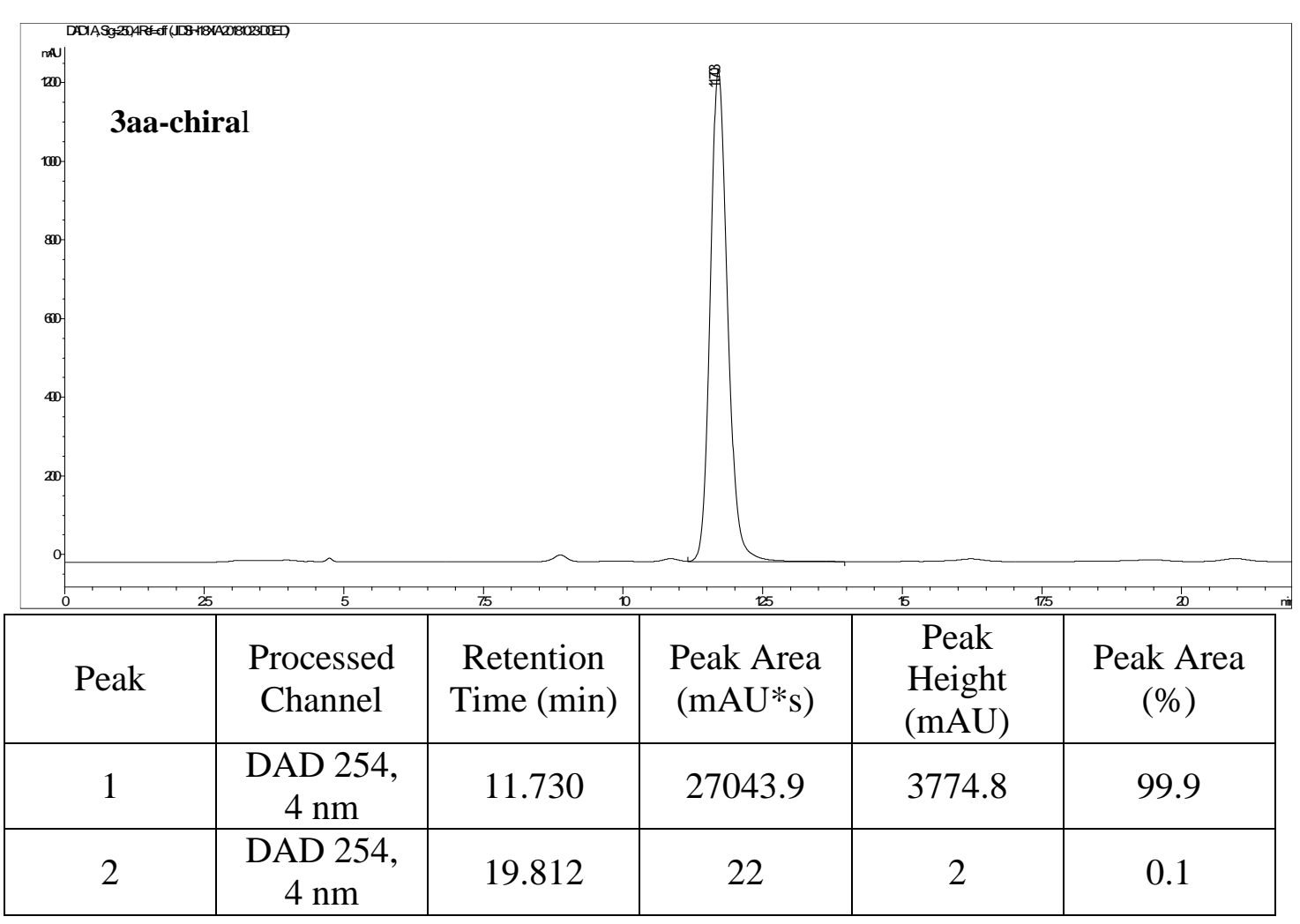




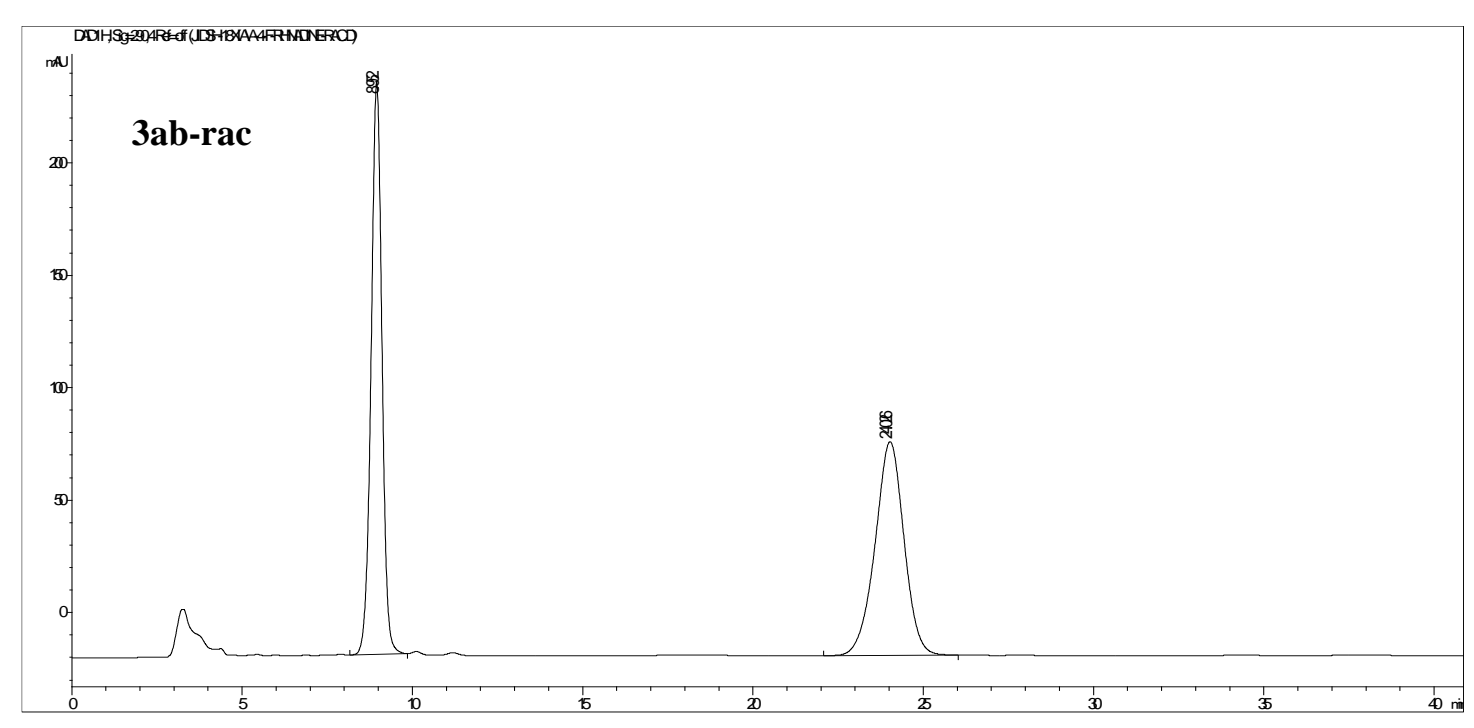

\begin{tabular}{|c|c|c|c|c|c|}
\hline Peak & $\begin{array}{c}\text { Processed } \\
\text { Channel }\end{array}$ & $\begin{array}{c}\text { Retention } \\
\text { Time (min) }\end{array}$ & $\begin{array}{c}\text { Peak Area } \\
\left.(\mathrm{mAU})^{*}\right)\end{array}$ & $\begin{array}{c}\text { Peak } \\
\text { Height } \\
(\mathrm{mAU})\end{array}$ & $\begin{array}{c}\text { Peak Area } \\
(\%)\end{array}$ \\
\hline 1 & $\begin{array}{c}\text { DAD 254, } \\
4 \mathrm{~nm}\end{array}$ & 8.952 & 5630.8 & 254.5 & 50.074 \\
\hline 2 & $\begin{array}{c}\text { DAD 254, } \\
4 \mathrm{~nm}\end{array}$ & 24.026 & 5614.2 & 95.1 & 49.926 \\
\hline
\end{tabular}

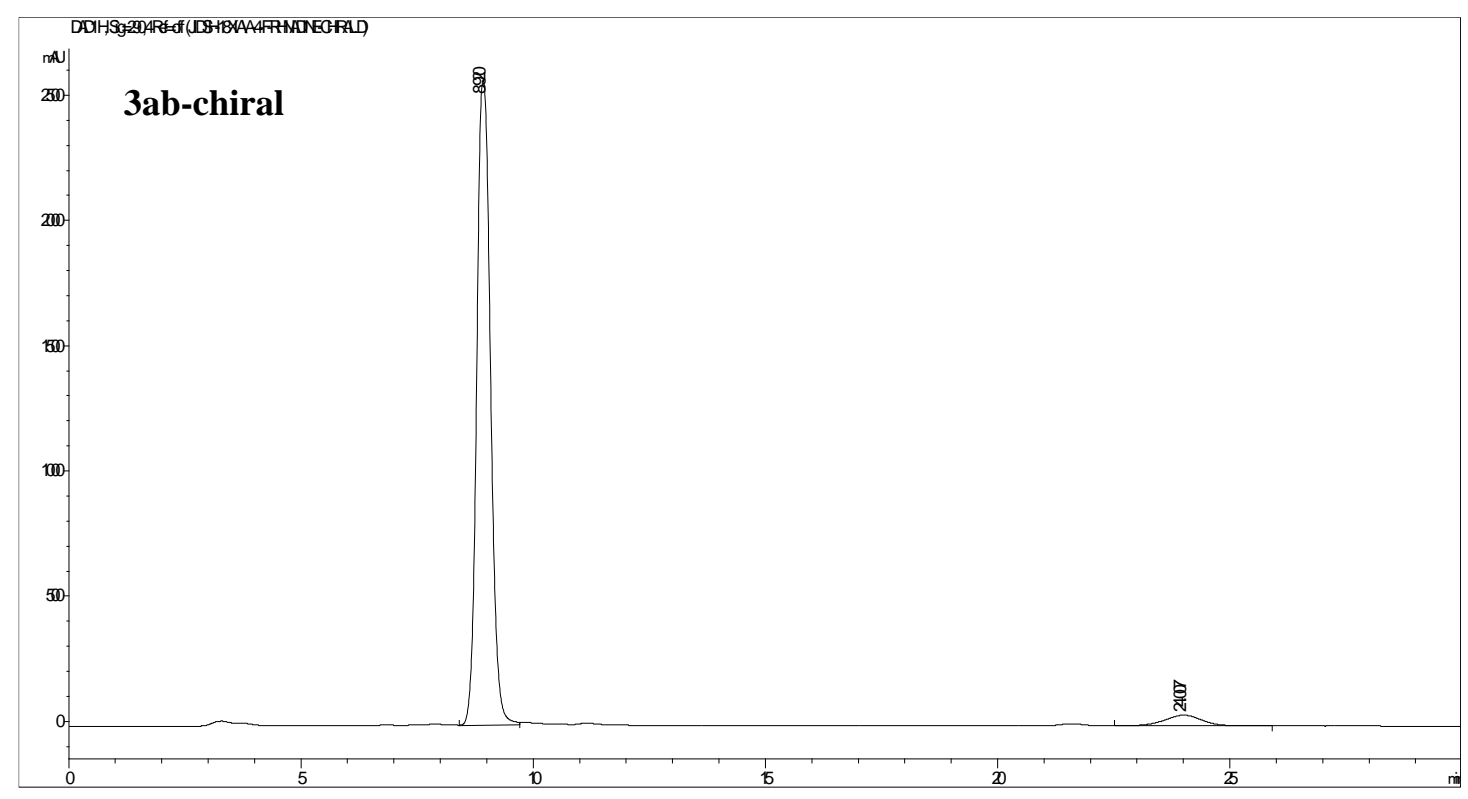

\begin{tabular}{|c|c|c|c|c|c|}
\hline Peak & $\begin{array}{c}\text { Processed } \\
\text { Channel }\end{array}$ & $\begin{array}{c}\text { Retention } \\
\text { Time (min) }\end{array}$ & $\begin{array}{c}\text { Peak Area } \\
(\mathrm{mAU} \text { s })\end{array}$ & $\begin{array}{c}\text { Peak } \\
\text { Height } \\
(\mathrm{mAU})\end{array}$ & $\begin{array}{c}\text { Peak Area } \\
(\%)\end{array}$ \\
\hline 1 & $\begin{array}{c}\text { DAD 254, } \\
4 \mathrm{~nm}\end{array}$ & 8.92 & 53080 & 2575 & 95.564 \\
\hline 2 & $\begin{array}{c}\text { DAD 254, } \\
4 \mathrm{~nm}\end{array}$ & 24.007 & 2463.8 & 43.2 & 4.436 \\
\hline
\end{tabular}




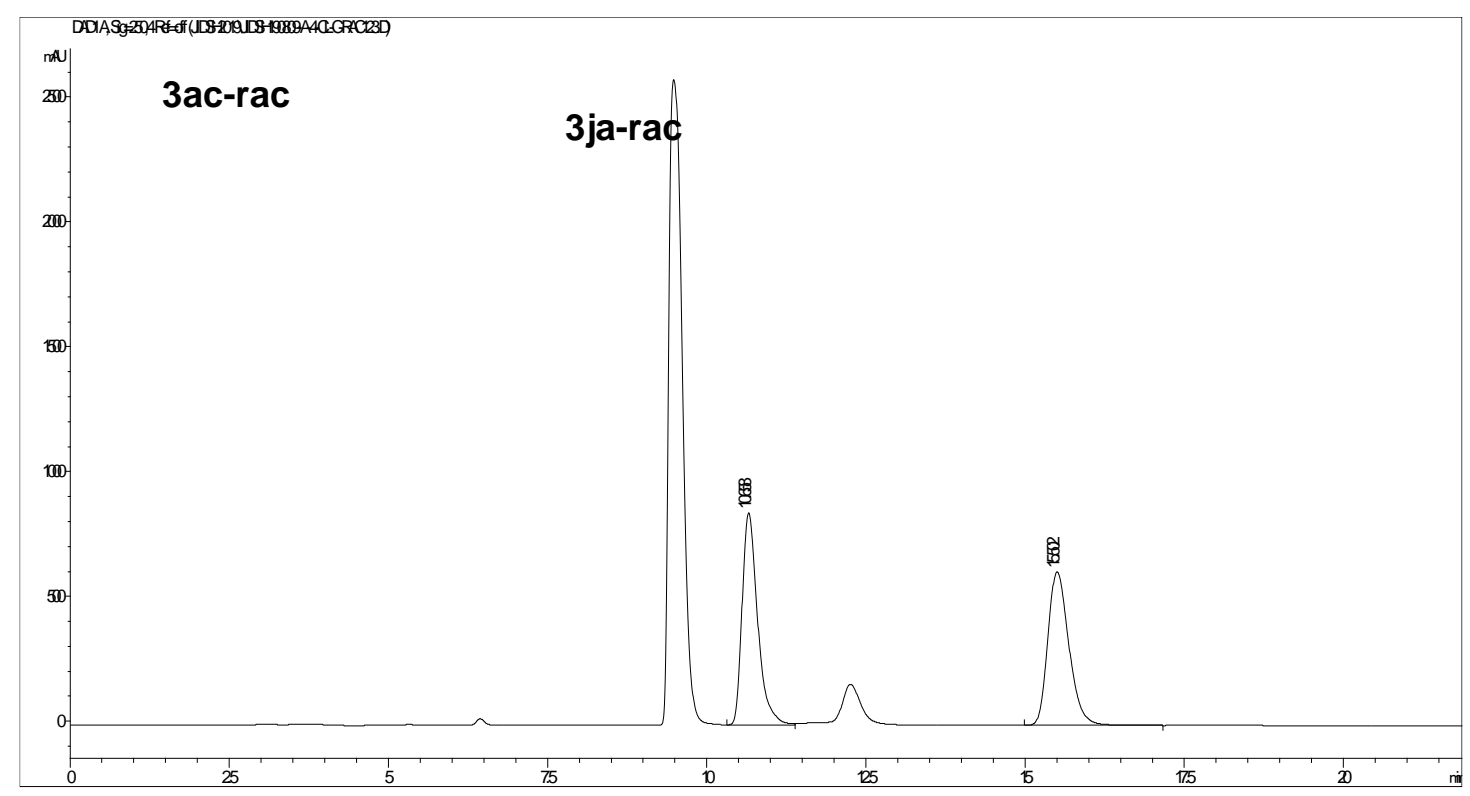

\begin{tabular}{|c|c|c|c|c|c|}
\hline Peak & $\begin{array}{c}\text { Processed } \\
\text { Channel }\end{array}$ & $\begin{array}{c}\text { Retention } \\
\text { Time (min) }\end{array}$ & $\begin{array}{c}\text { Peak Area } \\
\left(\mathrm{mAU}^{*} \mathrm{~s}\right)\end{array}$ & $\begin{array}{c}\text { Peak } \\
\text { Height } \\
(\mathrm{mAU})\end{array}$ & $\begin{array}{c}\text { Peak Area } \\
(\%)\end{array}$ \\
\hline 1 & $\begin{array}{c}\text { DAD 254, } \\
4 \mathrm{~nm}\end{array}$ & 10.658 & 14533.4 & 850.7 & 50.60 \\
\hline 2 & $\begin{array}{c}\text { DAD 254, } \\
4 \mathrm{~nm}\end{array}$ & 15.502 & 14188.4 & 614.6 & 49.40 \\
\hline
\end{tabular}

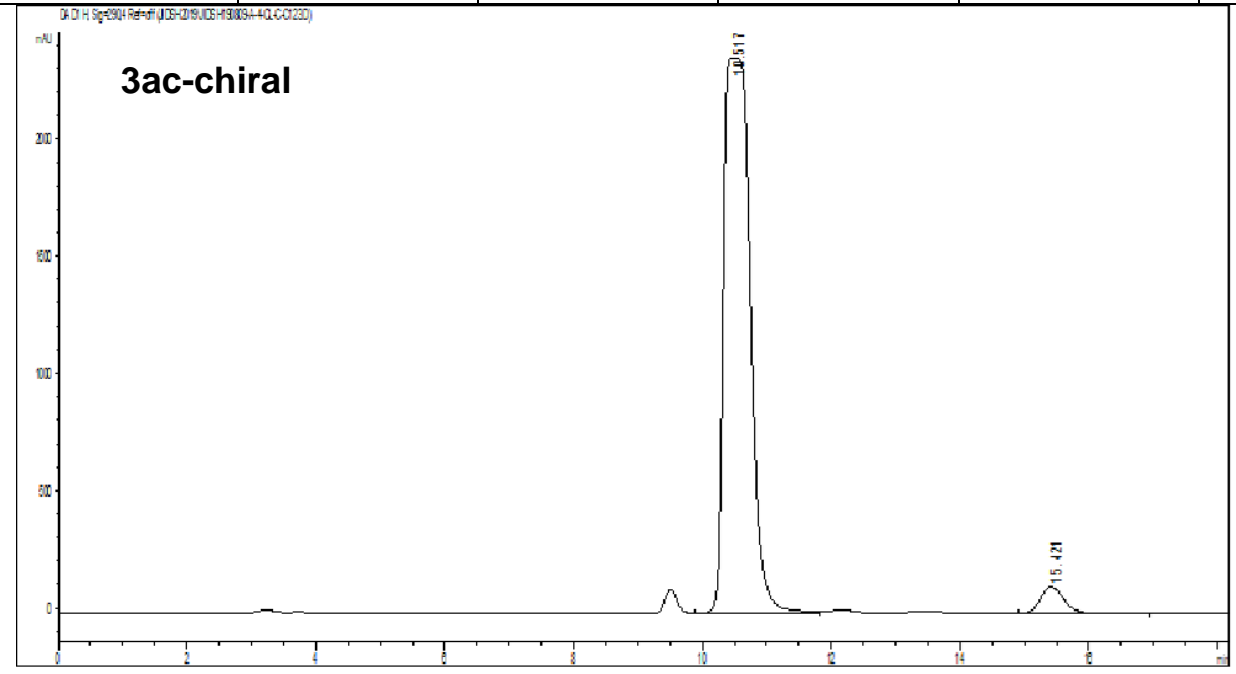

\begin{tabular}{|c|c|c|c|c|c|}
\hline Peak & $\begin{array}{c}\text { Processed } \\
\text { Channel }\end{array}$ & $\begin{array}{c}\text { Retention } \\
\text { Time (min) }\end{array}$ & $\begin{array}{c}\text { Peak Area } \\
\left.(\mathrm{mAU})^{*}\right)\end{array}$ & $\begin{array}{c}\text { Peak } \\
\text { Height } \\
(\mathrm{mAU})\end{array}$ & $\begin{array}{c}\text { Peak Area } \\
(\%)\end{array}$ \\
\hline 1 & $\begin{array}{c}\text { DAD 254, } \\
4 \mathrm{~nm}\end{array}$ & 10.517 & 67539 & 2366.6 & 95.96 \\
\hline 2 & $\begin{array}{c}\text { DAD 254, } \\
4 \mathrm{~nm}\end{array}$ & 15.421 & 2839.5 & 112.3 & 4.04 \\
\hline
\end{tabular}




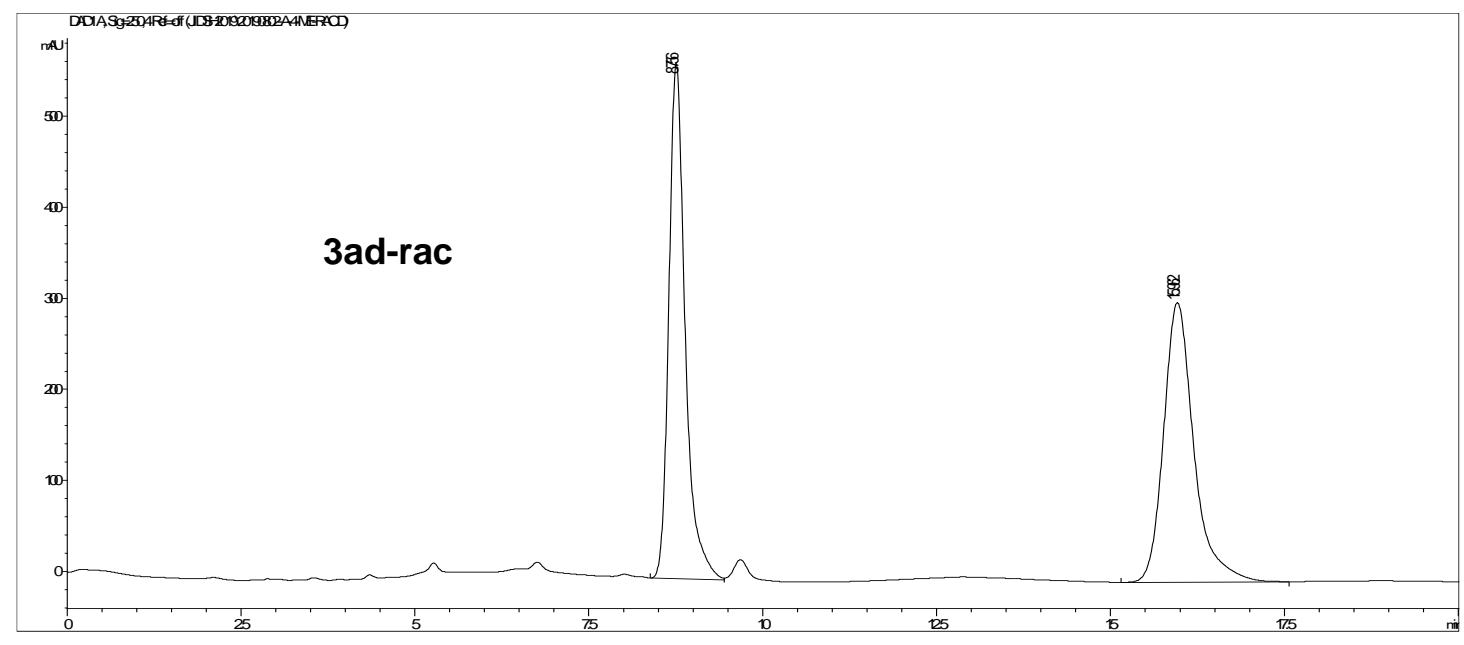

\begin{tabular}{|c|c|c|c|c|c|}
\hline Peak & $\begin{array}{c}\text { Processed } \\
\text { Channel }\end{array}$ & $\begin{array}{c}\text { Retention } \\
\text { Time (min) }\end{array}$ & $\begin{array}{c}\text { Peak Area } \\
\left(\mathrm{mAU}^{*} \mathrm{~s}\right)\end{array}$ & $\begin{array}{c}\text { Peak } \\
\text { Height } \\
(\mathrm{mAU})\end{array}$ & $\begin{array}{c}\text { Peak Area } \\
(\%)\end{array}$ \\
\hline 1 & $\begin{array}{c}\text { DAD 254, } \\
4 \mathrm{~nm}\end{array}$ & 8.756 & 9263.6 & 566.2 & 49.99 \\
\hline 2 & $\begin{array}{c}\text { DAD 254, } \\
4 \mathrm{~nm}\end{array}$ & 15.962 & 9266.3 & 306.9 & 50.01 \\
\hline
\end{tabular}

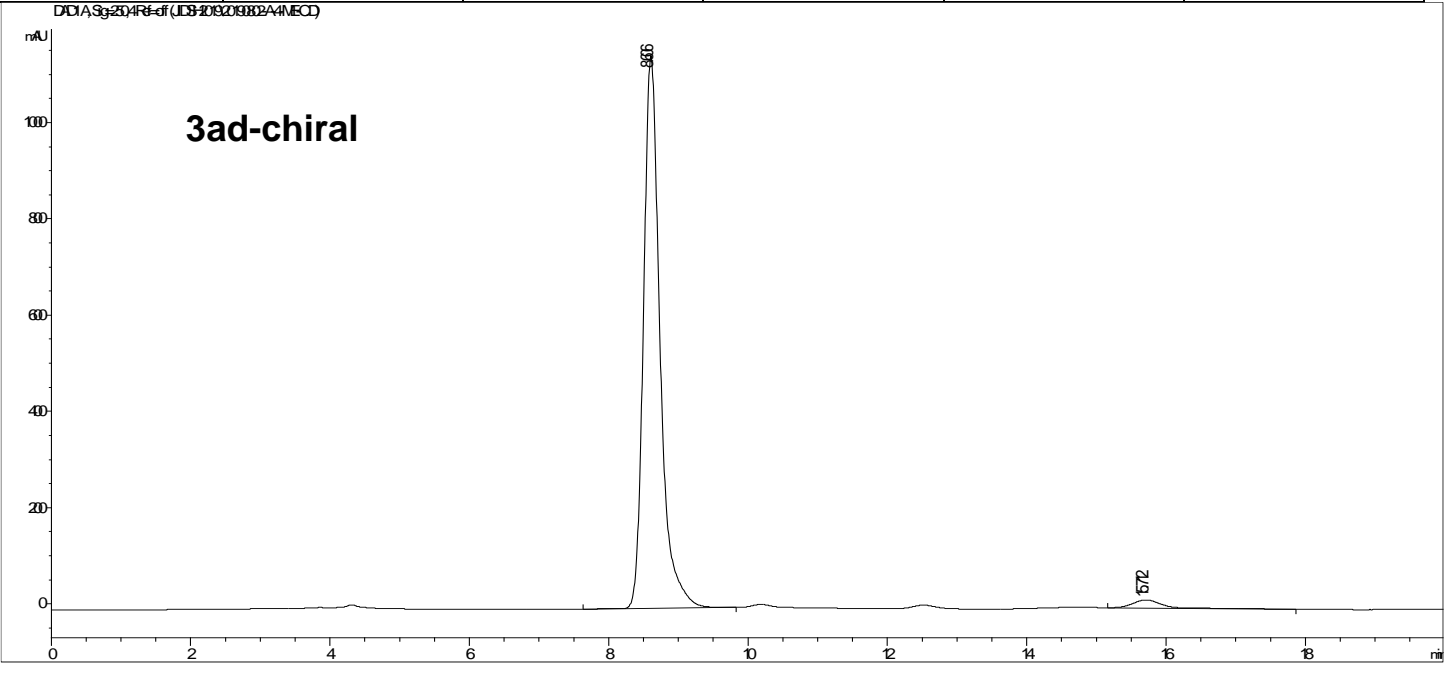

\begin{tabular}{|c|c|c|c|c|c|}
\hline Peak & $\begin{array}{c}\text { Processed } \\
\text { Channel }\end{array}$ & $\begin{array}{c}\text { Retention } \\
\text { Time (min) }\end{array}$ & $\begin{array}{c}\text { Peak Area } \\
\left.(\mathrm{mAU})^{*}\right)\end{array}$ & $\begin{array}{c}\text { Peak } \\
\text { Height } \\
(\mathrm{mAU})\end{array}$ & $\begin{array}{c}\text { Peak Area } \\
(\%)\end{array}$ \\
\hline 1 & $\begin{array}{c}\text { DAD 254, } \\
4 \mathrm{~nm}\end{array}$ & 8.606 & 10348.3 & 629.7 & 97.10 \\
\hline 2 & $\begin{array}{c}\text { DAD 254, } \\
4 \mathrm{~nm}\end{array}$ & 15.714 & 15.714 & 9 & 3.90 \\
\hline
\end{tabular}




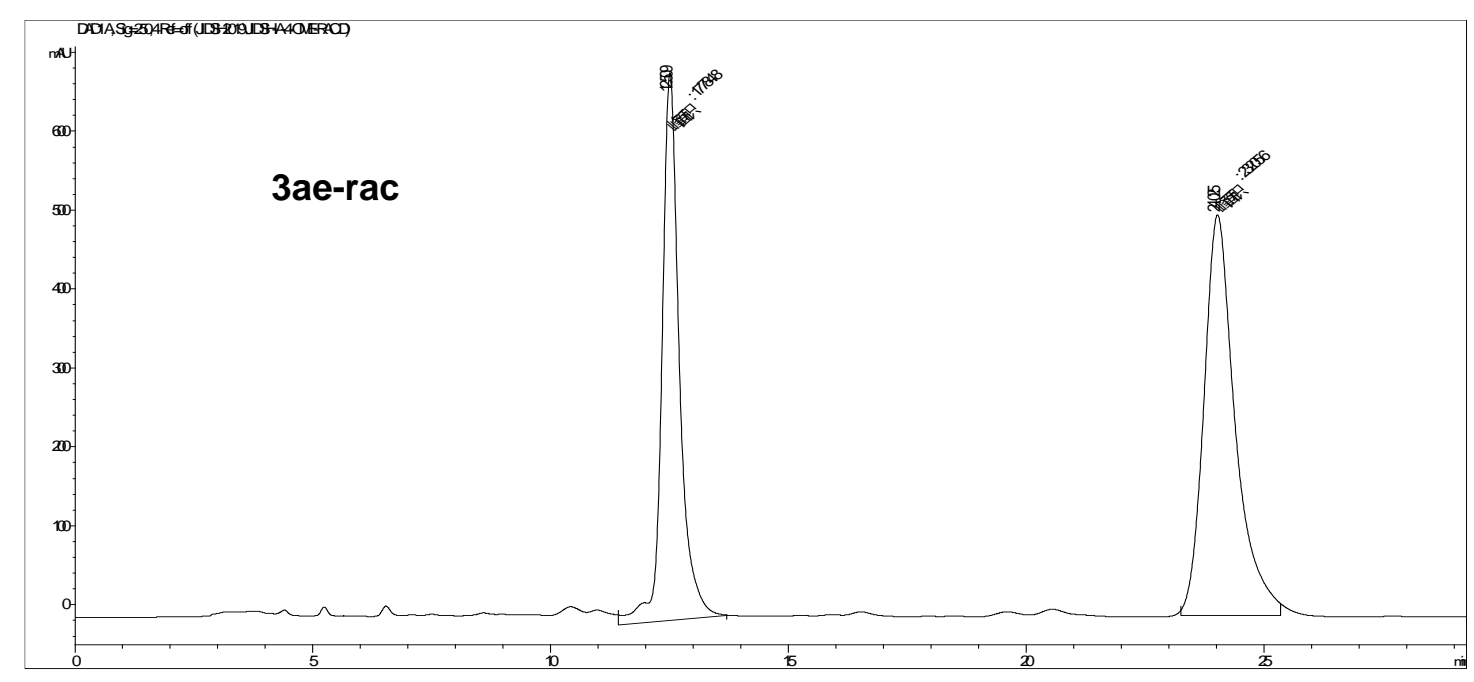

\begin{tabular}{|c|c|c|c|c|c|}
\hline Peak & $\begin{array}{c}\text { Processed } \\
\text { Channel }\end{array}$ & $\begin{array}{c}\text { Retention } \\
\text { Time (min) }\end{array}$ & $\begin{array}{c}\text { Peak Area } \\
(\mathrm{mAU} \text { * })\end{array}$ & $\begin{array}{c}\text { Peak } \\
\text { Height } \\
(\mathrm{mAU})\end{array}$ & $\begin{array}{c}\text { Peak Area } \\
(\%)\end{array}$ \\
\hline 1 & $\begin{array}{c}\text { DAD 254, } \\
4 \mathrm{~nm}\end{array}$ & 12.509 & 17784.8 & 693.9 & 48.88 \\
\hline 2 & $\begin{array}{c}\text { DAD 254, } \\
4 \mathrm{~nm}\end{array}$ & 24.025 & 20205.6 & 507.6 & 51.12 \\
\hline
\end{tabular}

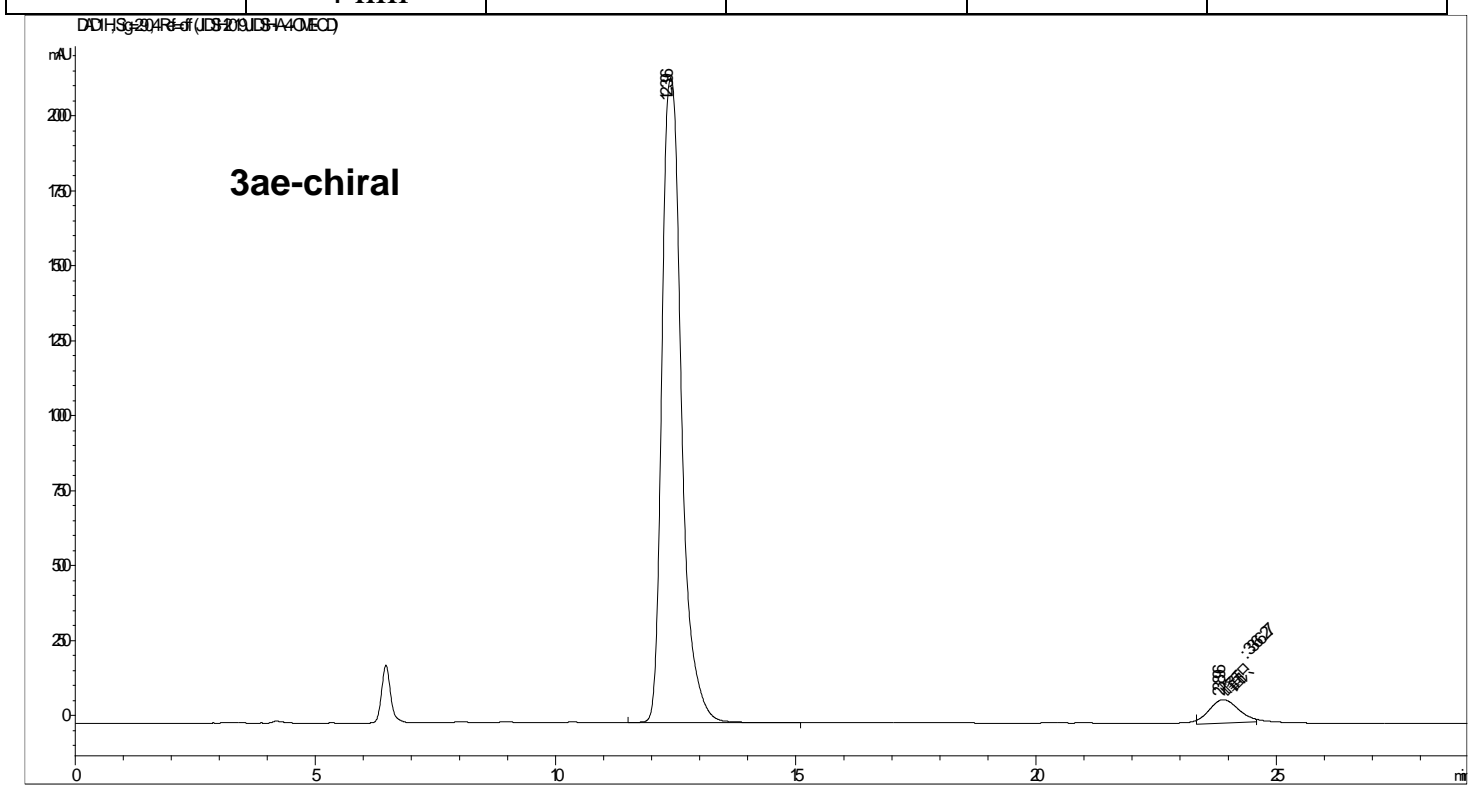

\begin{tabular}{|c|c|c|c|c|c|}
\hline Peak & $\begin{array}{c}\text { Processed } \\
\text { Channel }\end{array}$ & $\begin{array}{c}\text { Retention } \\
\text { Time (min) }\end{array}$ & $\begin{array}{c}\text { Peak Area } \\
\left.(\mathrm{mAU})^{*}\right)\end{array}$ & $\begin{array}{c}\text { Peak } \\
\text { Height } \\
(\mathrm{mAU})\end{array}$ & $\begin{array}{c}\text { Peak Area } \\
(\%)\end{array}$ \\
\hline 1 & $\begin{array}{c}\text { DAD 254, } \\
4 \mathrm{~nm}\end{array}$ & 12.396 & 60734 & 2151.7 & 95.01 \\
\hline 2 & $\begin{array}{c}\text { DAD 254, } \\
4 \mathrm{~nm}\end{array}$ & 23.896 & 3366.3 & 78.6 & 4.99 \\
\hline
\end{tabular}




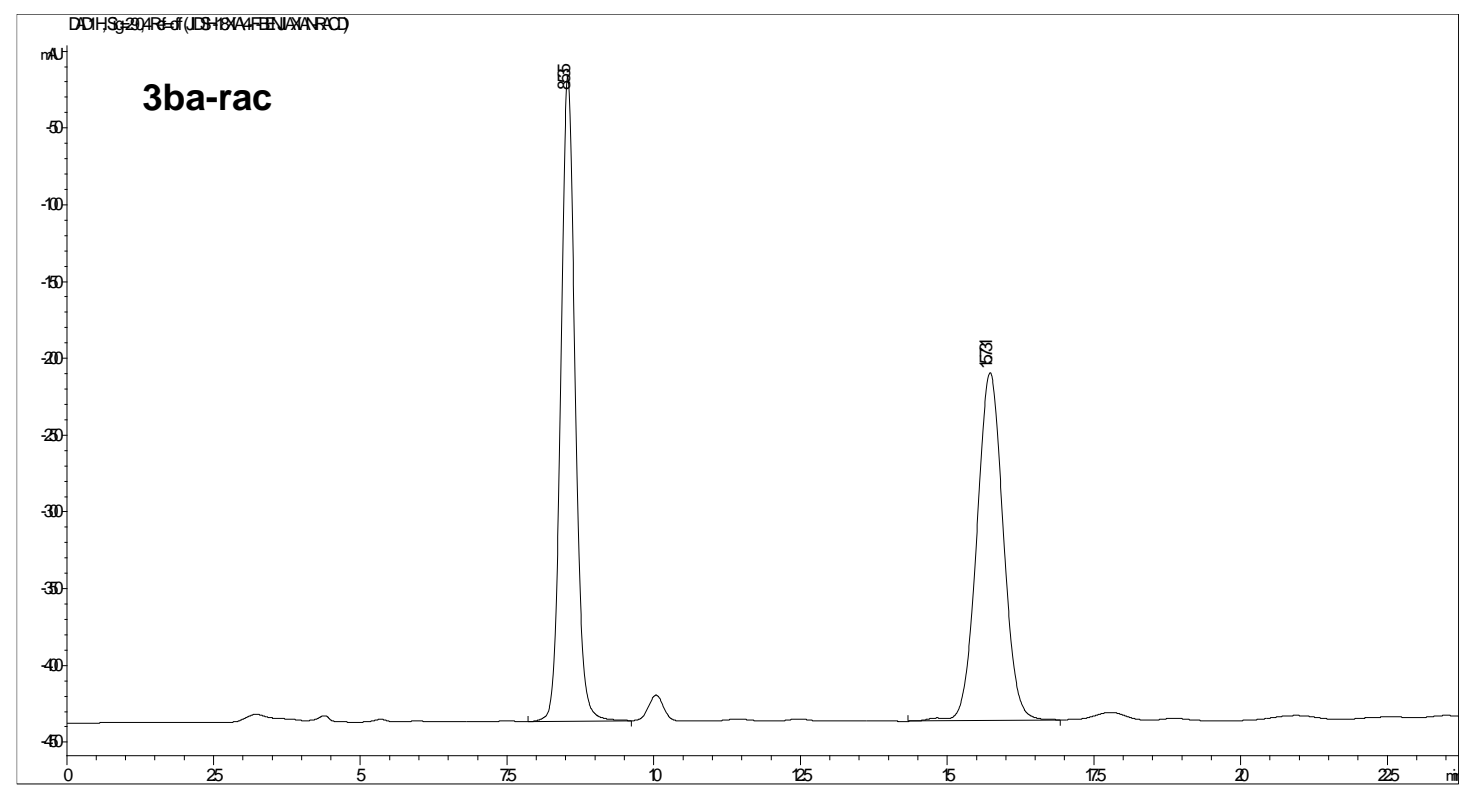

\begin{tabular}{|c|c|c|c|c|c|}
\hline Peak & $\begin{array}{c}\text { Processed } \\
\text { Channel }\end{array}$ & $\begin{array}{c}\text { Retention } \\
\text { Time (min) }\end{array}$ & $\begin{array}{c}\text { Peak Area } \\
(\mathrm{mAU} \text { ) })\end{array}$ & $\begin{array}{c}\text { Peak } \\
\text { Height } \\
(\mathrm{mAU})\end{array}$ & $\begin{array}{c}\text { Peak Area } \\
(\%)\end{array}$ \\
\hline 1 & $\begin{array}{c}\text { DAD 254, } \\
4 \mathrm{~nm}\end{array}$ & 8.535 & 419.6 & 3774.8 & 50.285 \\
\hline 2 & $\begin{array}{c}\text { DAD 254, } \\
4 \mathrm{~nm}\end{array}$ & 15.731 & 226.4 & 88.4 & 49.715 \\
\hline
\end{tabular}

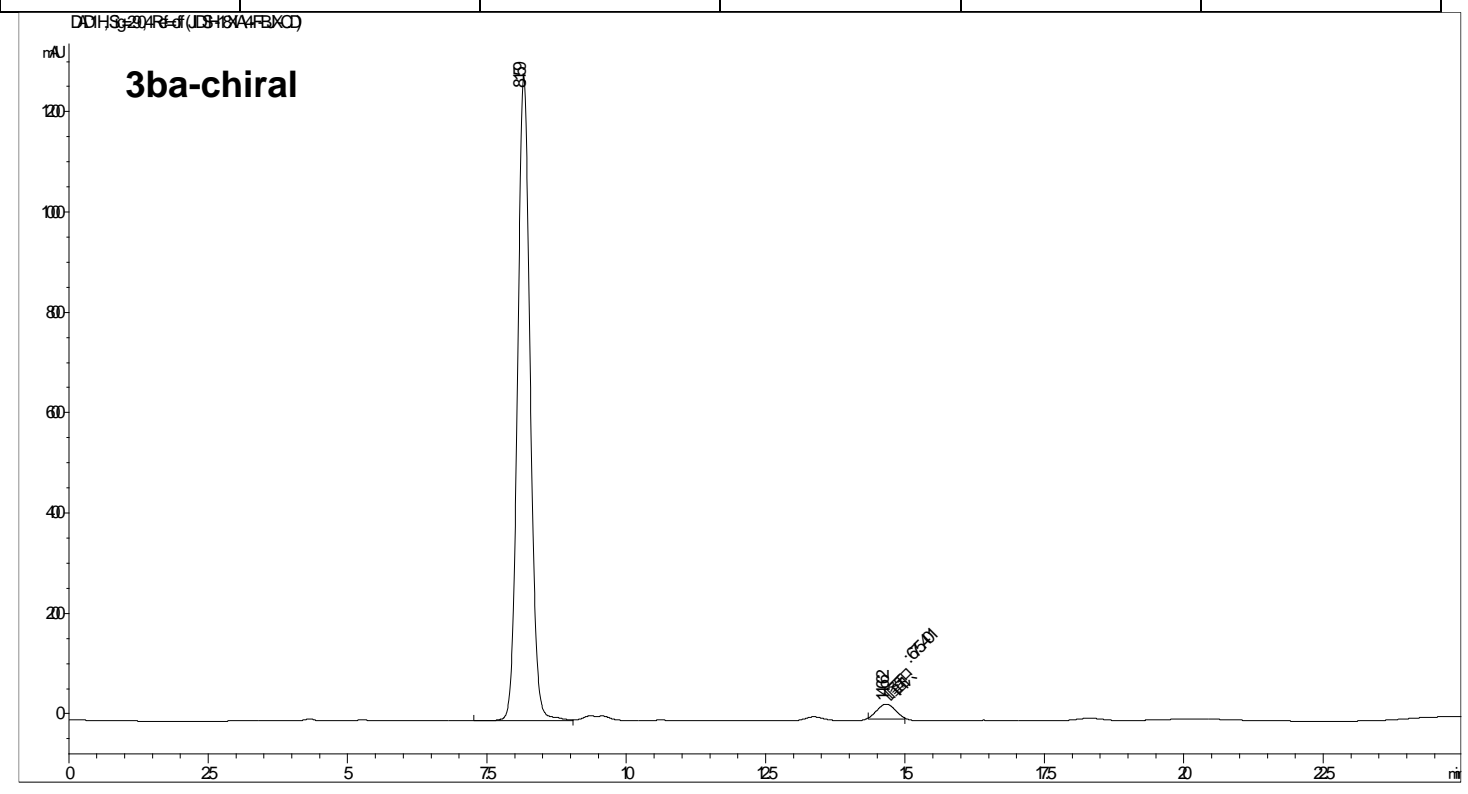

\begin{tabular}{|c|c|c|c|c|c|}
\hline Peak & $\begin{array}{c}\text { Processed } \\
\text { Channel }\end{array}$ & $\begin{array}{c}\text { Retention } \\
\text { Time (min) }\end{array}$ & $\begin{array}{c}\text { Peak Area } \\
(\mathrm{mAU} \text { * })\end{array}$ & $\begin{array}{c}\text { Peak } \\
\text { Height } \\
(\mathrm{mAU})\end{array}$ & $\begin{array}{c}\text { Peak Area } \\
(\%)\end{array}$ \\
\hline 1 & $\begin{array}{c}\text { DAD 254, } \\
4 \mathrm{~nm}\end{array}$ & 8.159 & 20298.9 & 1287.2 & 96.780 \\
\hline 2 & $\begin{array}{c}\text { DAD 254, } \\
4 \mathrm{~nm}\end{array}$ & 14.662 & 675.4 & 29.8 & 3.220 \\
\hline
\end{tabular}




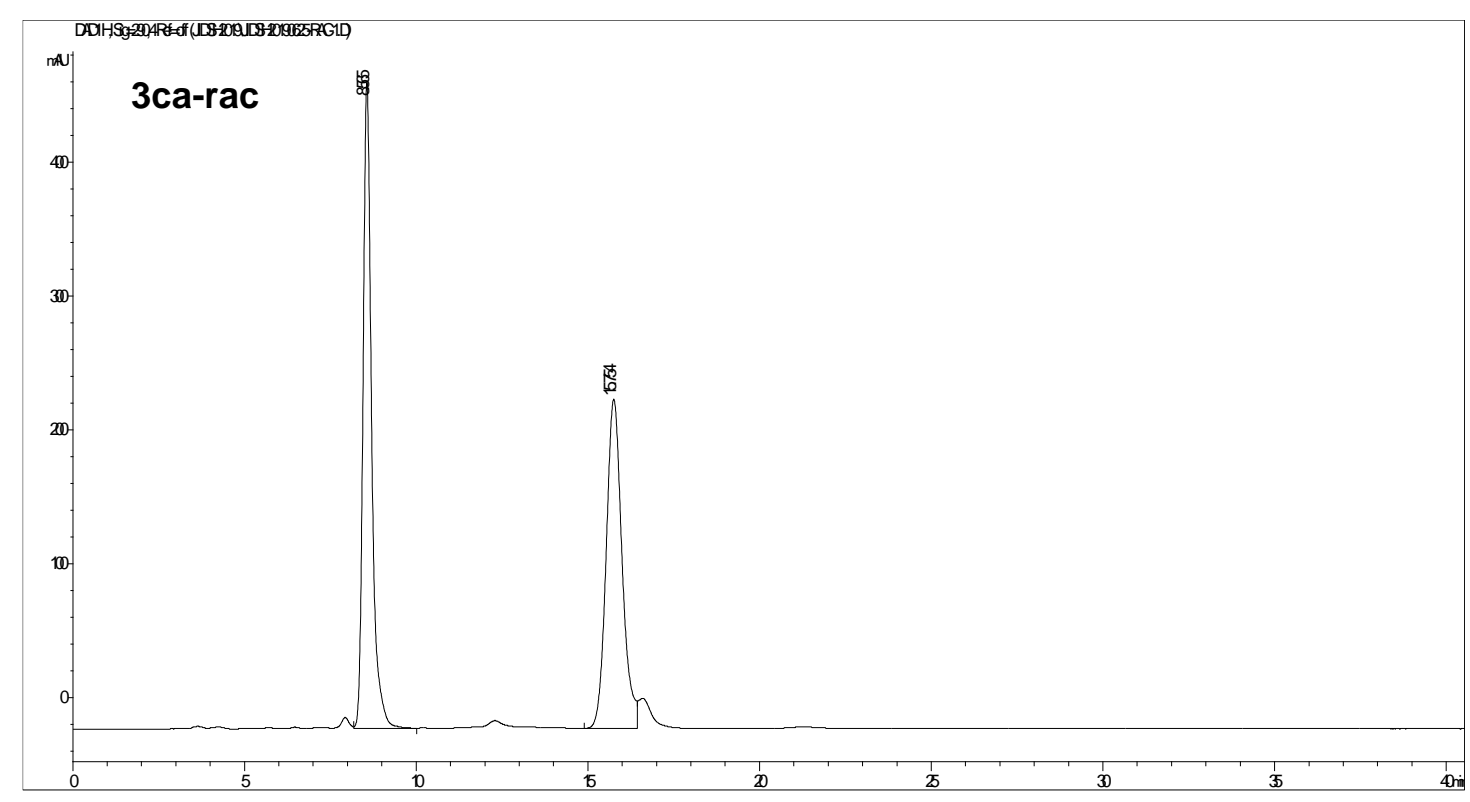

\begin{tabular}{|c|c|c|c|c|c|}
\hline Peak & $\begin{array}{c}\text { Processed } \\
\text { Channel }\end{array}$ & $\begin{array}{c}\text { Retention } \\
\text { Time (min) }\end{array}$ & $\begin{array}{c}\text { Peak Area } \\
\left(\text { Mau*s }^{*}\right.\end{array}$ & $\begin{array}{c}\text { Peak } \\
\text { Height } \\
(\mathrm{mAU})\end{array}$ & $\begin{array}{c}\text { Peak Area } \\
(\%)\end{array}$ \\
\hline 1 & $\begin{array}{c}\text { DAD 254, } \\
4 \mathrm{~nm}\end{array}$ & 8.565 & 8352.7 & 482.1 & 50.472 \\
\hline 2 & $\begin{array}{c}\text { DAD 254, } \\
4 \mathrm{~nm}\end{array}$ & 15.754 & 7874.2 & 245.9 & 48.528 \\
\hline
\end{tabular}

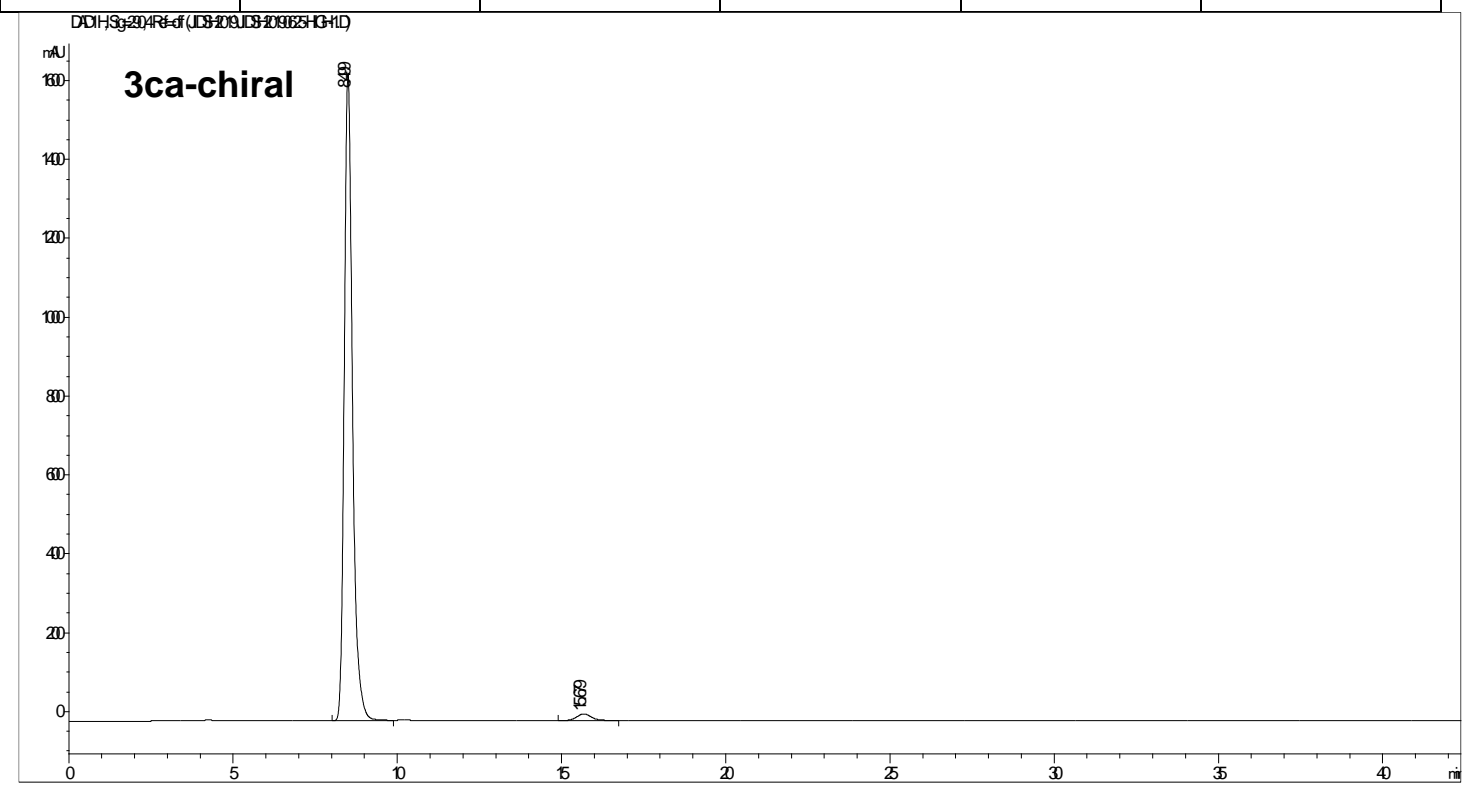

\begin{tabular}{|c|c|c|c|c|c|}
\hline Peak & $\begin{array}{c}\text { Processed } \\
\text { Channel }\end{array}$ & $\begin{array}{c}\text { Retention } \\
\text { Time (min) }\end{array}$ & $\begin{array}{c}\text { Peak Area } \\
(\text { Mau*s })\end{array}$ & $\begin{array}{c}\text { Peak } \\
\text { Height } \\
(\mathrm{mAU})\end{array}$ & $\begin{array}{c}\text { Peak Area } \\
(\%)\end{array}$ \\
\hline 1 & $\begin{array}{c}\text { DAD 254, } \\
4 \mathrm{~nm}\end{array}$ & 8.499 & 28660.2 & 1636.9 & 98.143 \\
\hline 2 & $\begin{array}{c}\text { DAD 254, } \\
4 \mathrm{~nm}\end{array}$ & 15.679 & 542.5 & 16.9 & 1.857 \\
\hline
\end{tabular}




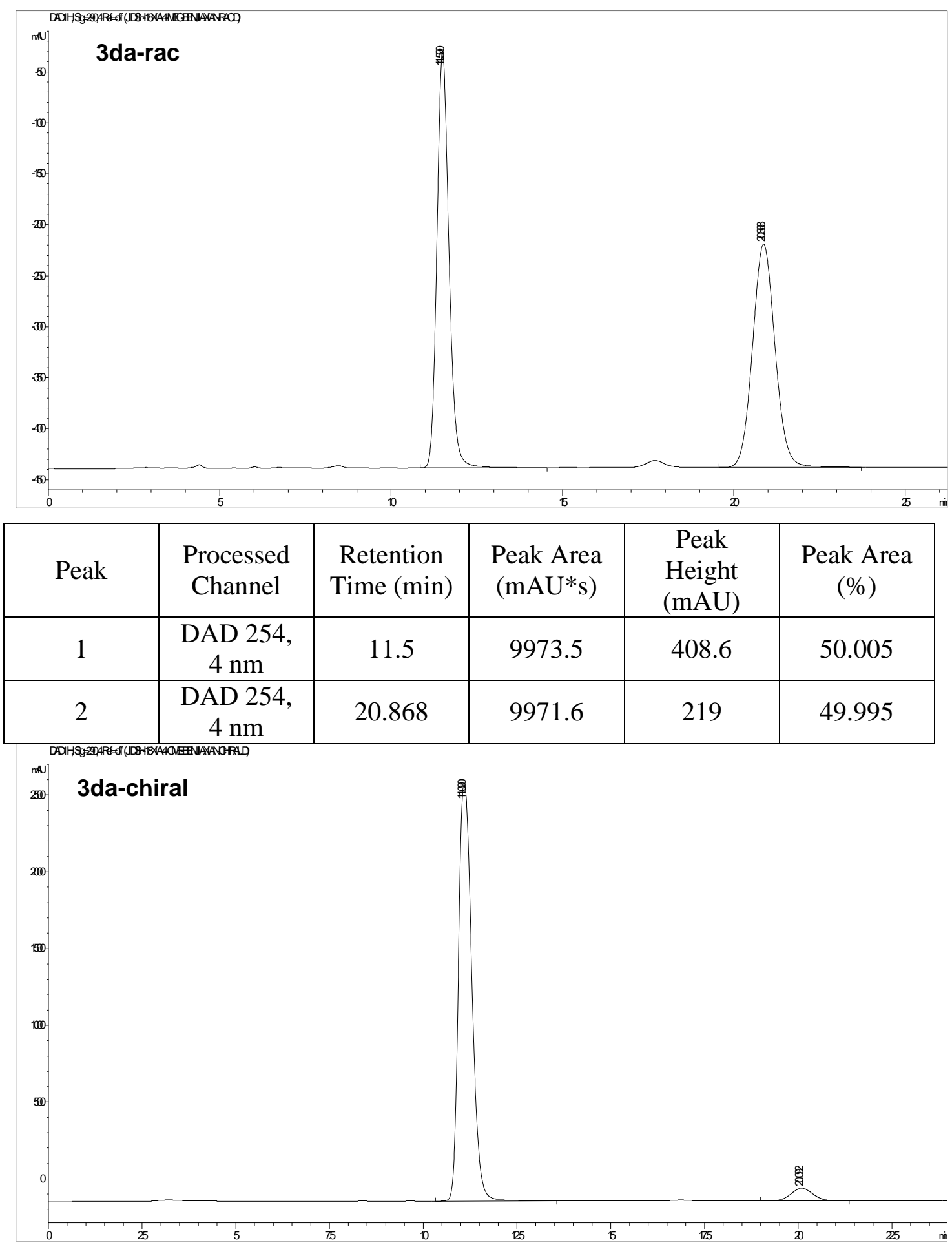

\begin{tabular}{|c|c|c|c|c|c|}
\hline Peak & $\begin{array}{c}\text { Processed } \\
\text { Channel }\end{array}$ & $\begin{array}{c}\text { Retention } \\
\text { Time (min) }\end{array}$ & $\begin{array}{c}\text { Peak Area } \\
\left(\mathrm{mAU}^{*} \mathrm{~s}\right)\end{array}$ & $\begin{array}{c}\text { Peak } \\
\text { Height } \\
(\mathrm{mAU})\end{array}$ & $\begin{array}{c}\text { Peak Area } \\
(\%)\end{array}$ \\
\hline 1 & $\begin{array}{c}\text { DAD 254, } \\
4 \mathrm{~nm}\end{array}$ & 11.09 & 67241.9 & 2714.5 & 95.232 \\
\hline 2 & $\begin{array}{c}\text { DAD 254, } \\
4 \mathrm{~nm}\end{array}$ & 20.092 & 3366.6 & 82.6 & 4.768 \\
\hline
\end{tabular}




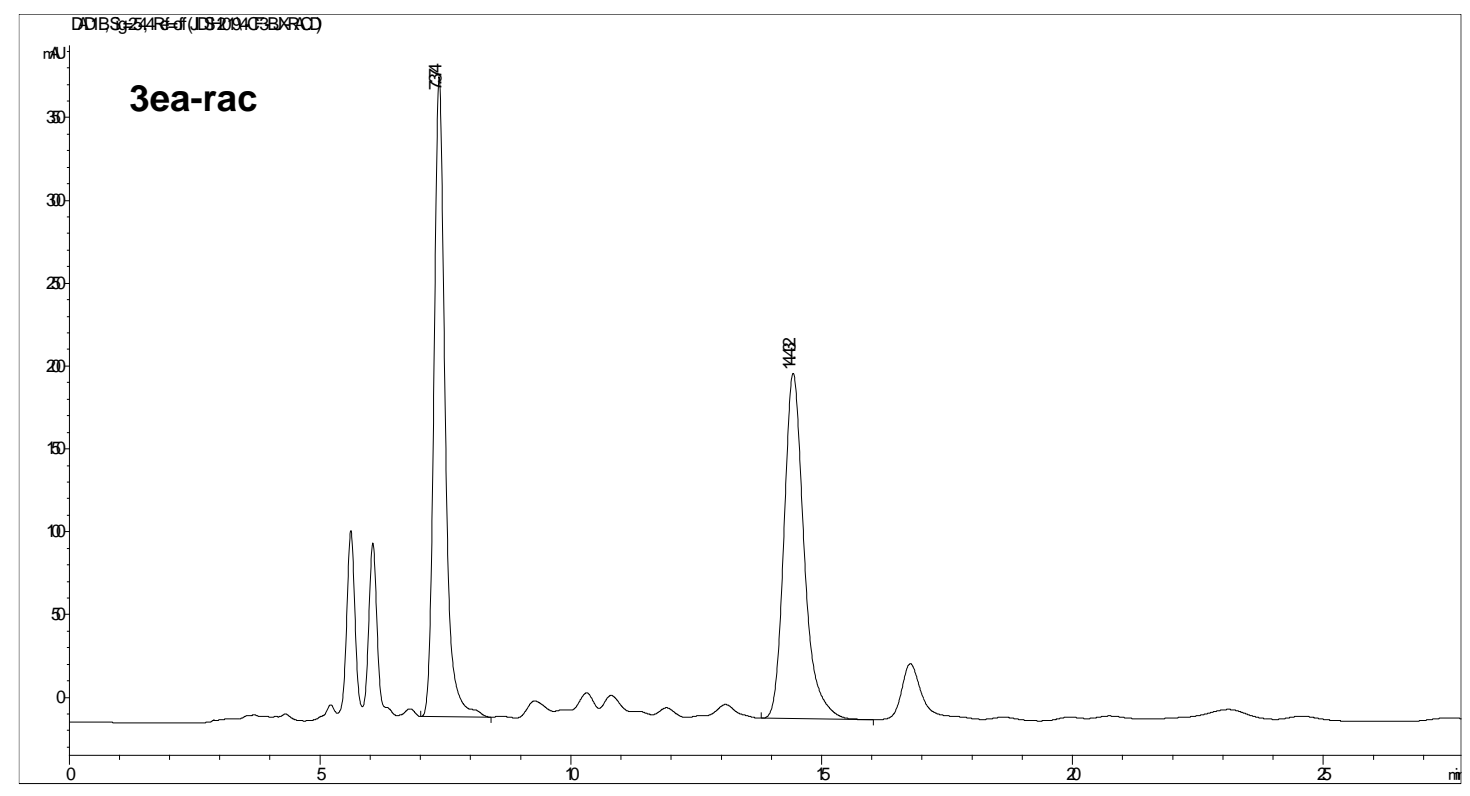

\begin{tabular}{|c|c|c|c|c|c|}
\hline Peak & $\begin{array}{c}\text { Processed } \\
\text { Channel }\end{array}$ & $\begin{array}{c}\text { Retention } \\
\text { Time (min) }\end{array}$ & $\begin{array}{c}\text { Peak Area } \\
(\mathrm{mAU} \text { s })\end{array}$ & $\begin{array}{c}\text { Peak } \\
\text { Height } \\
(\mathrm{mAU})\end{array}$ & $\begin{array}{c}\text { Peak Area } \\
(\%)\end{array}$ \\
\hline 1 & $\begin{array}{c}\text { DAD 254, } \\
4 \mathrm{~nm}\end{array}$ & 7.374 & 5980.8 & 386.3 & 50.79 \\
\hline 2 & $\begin{array}{c}\text { DAD 254, } \\
4 \mathrm{~nm}\end{array}$ & 14.342 & 5794.6 & 208.5 & 49.21 \\
\hline
\end{tabular}

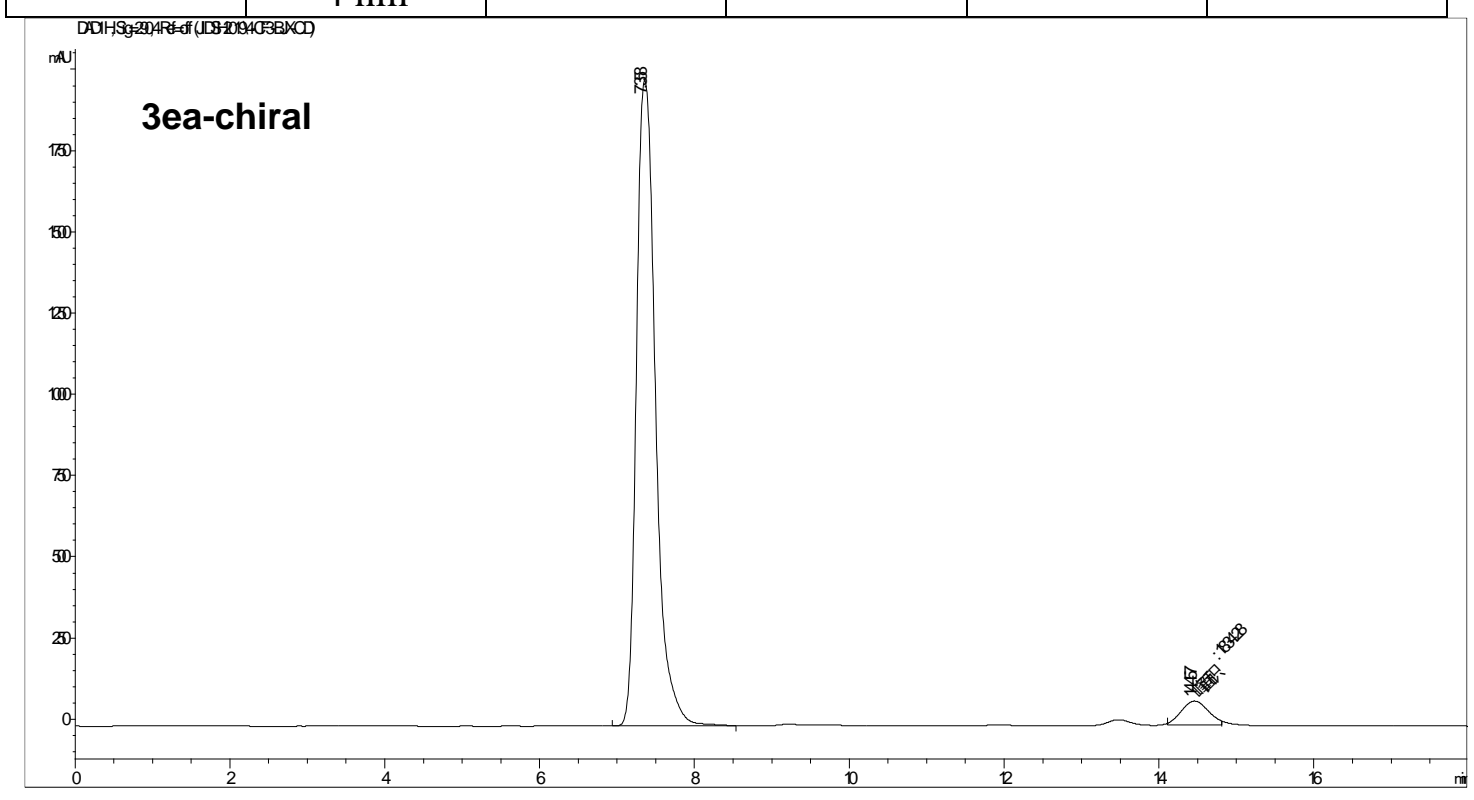

\begin{tabular}{|c|c|c|c|c|c|}
\hline Peak & $\begin{array}{c}\text { Processed } \\
\text { Channel }\end{array}$ & $\begin{array}{c}\text { Retention } \\
\text { Time (min) }\end{array}$ & $\begin{array}{c}\text { Peak Area } \\
(\mathrm{mAU} \text { * })\end{array}$ & $\begin{array}{c}\text { Peak } \\
\text { Height } \\
(\mathrm{mAU})\end{array}$ & $\begin{array}{c}\text { Peak Area } \\
(\%)\end{array}$ \\
\hline 1 & $\begin{array}{c}\text { DAD 254, } \\
4 \mathrm{~nm}\end{array}$ & 7.358 & 34113 & 1986.6 & 95 \\
\hline 2 & $\begin{array}{c}\text { DAD 254, } \\
4 \mathrm{~nm}\end{array}$ & 14.457 & 1834.3 & 74.8 & 5 \\
\hline
\end{tabular}



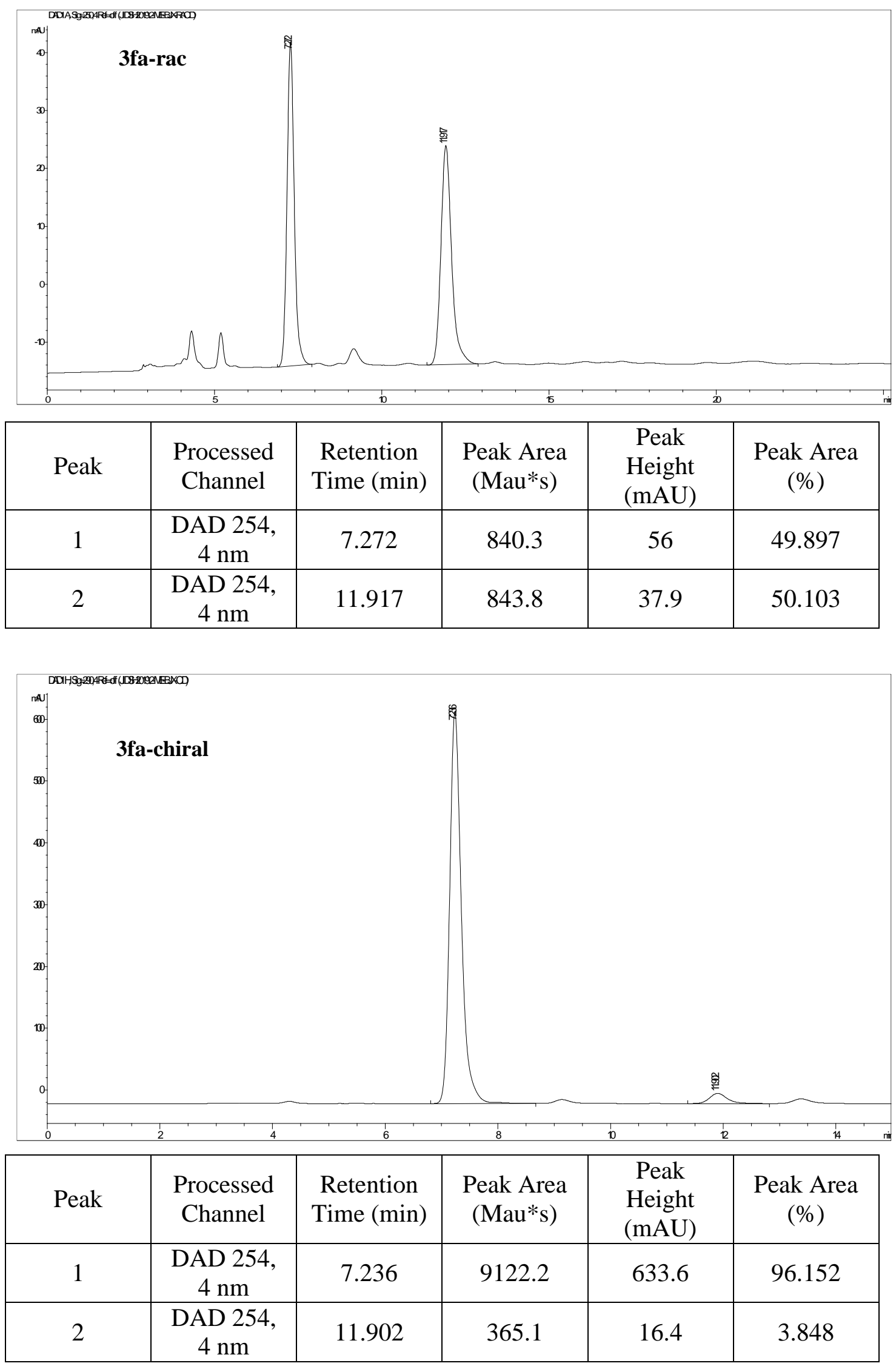


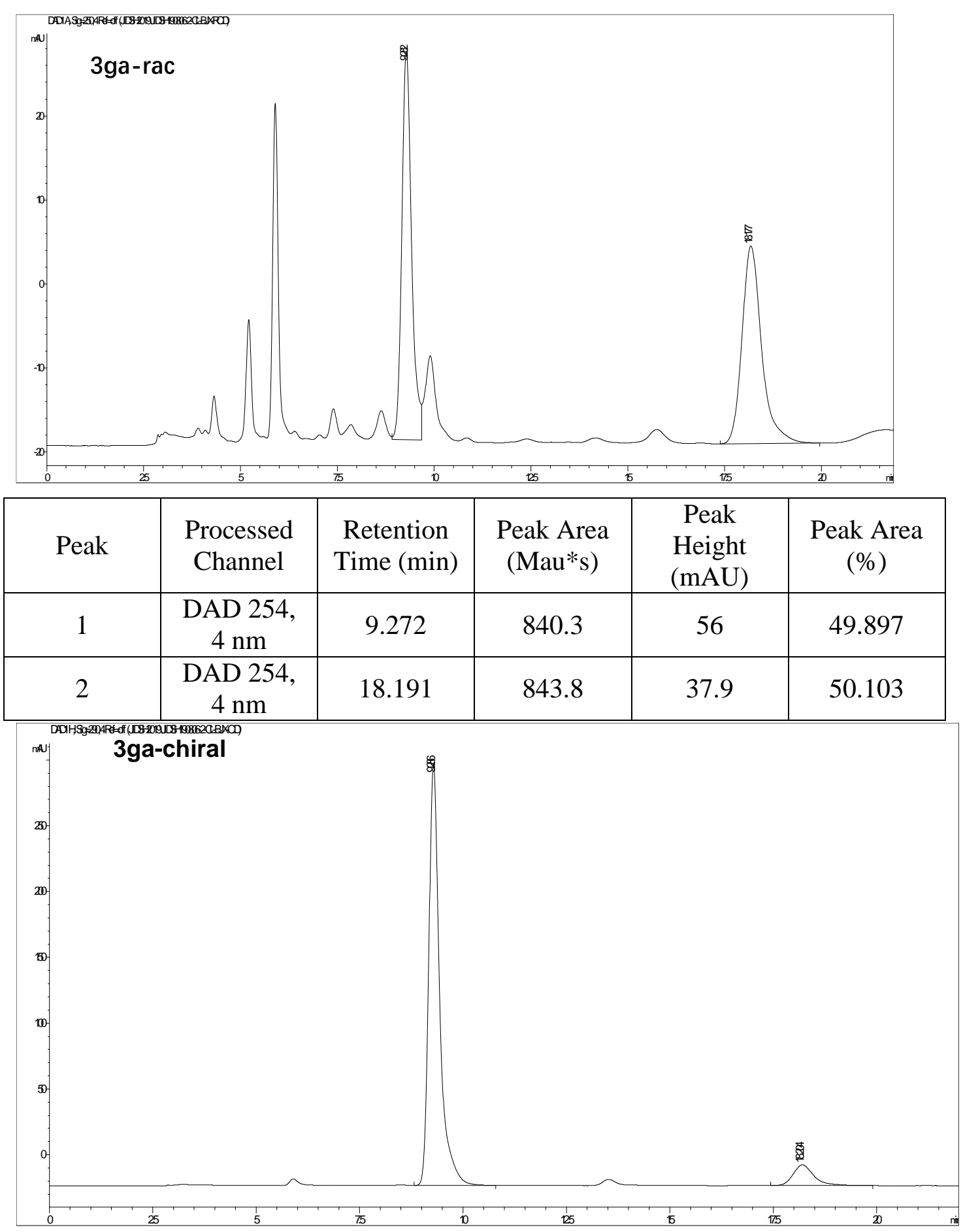

\begin{tabular}{|c|c|c|c|c|c|}
\hline Peak & $\begin{array}{c}\text { Processed } \\
\text { Channel }\end{array}$ & $\begin{array}{c}\text { Retention } \\
\text { Time (min) }\end{array}$ & $\begin{array}{c}\text { Peak Area } \\
\left(\mathrm{mAU}^{*} \mathrm{~s}\right)\end{array}$ & $\begin{array}{c}\text { Peak } \\
\text { Height } \\
(\mathrm{mAU})\end{array}$ & $\begin{array}{c}\text { Peak Area } \\
(\%)\end{array}$ \\
\hline 1 & $\begin{array}{c}\text { DAD 254, } \\
4 \mathrm{~nm}\end{array}$ & 9.26 & 13332 & 725.2 & 92.3 \\
\hline 2 & $\begin{array}{c}\text { DAD 254, } \\
4 \mathrm{~nm}\end{array}$ & 18.09 & 1000.1 & 38.6 & 7.7 \\
\hline
\end{tabular}




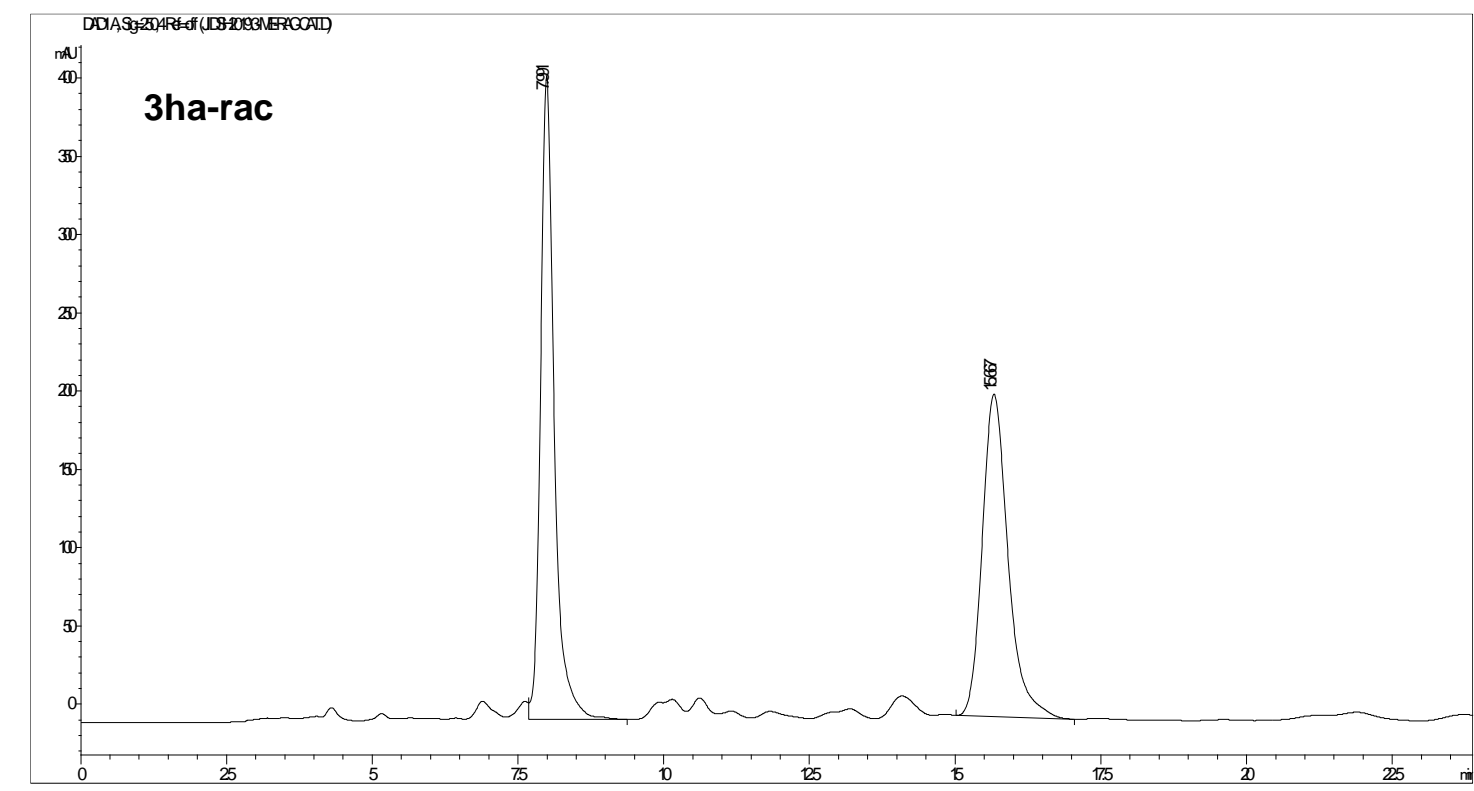

\begin{tabular}{|c|c|c|c|c|c|}
\hline Peak & $\begin{array}{c}\text { Processed } \\
\text { Channel }\end{array}$ & $\begin{array}{c}\text { Retention } \\
\text { Time (min) }\end{array}$ & $\begin{array}{c}\text { Peak Area } \\
(\mathrm{mAU} \text { ) })\end{array}$ & $\begin{array}{c}\text { Peak } \\
\text { Height } \\
(\mathrm{mAU})\end{array}$ & $\begin{array}{c}\text { Peak Area } \\
(\%)\end{array}$ \\
\hline 1 & $\begin{array}{c}\text { DAD 254, } \\
4 \mathrm{~nm}\end{array}$ & 7.99 & 6721.8 & 410.7 & 50.46 \\
\hline 2 & $\begin{array}{c}\text { DAD 254, } \\
4 \mathrm{~nm}\end{array}$ & 15.66 & 6356.7 & 206.2 & 49.54 \\
\hline
\end{tabular}

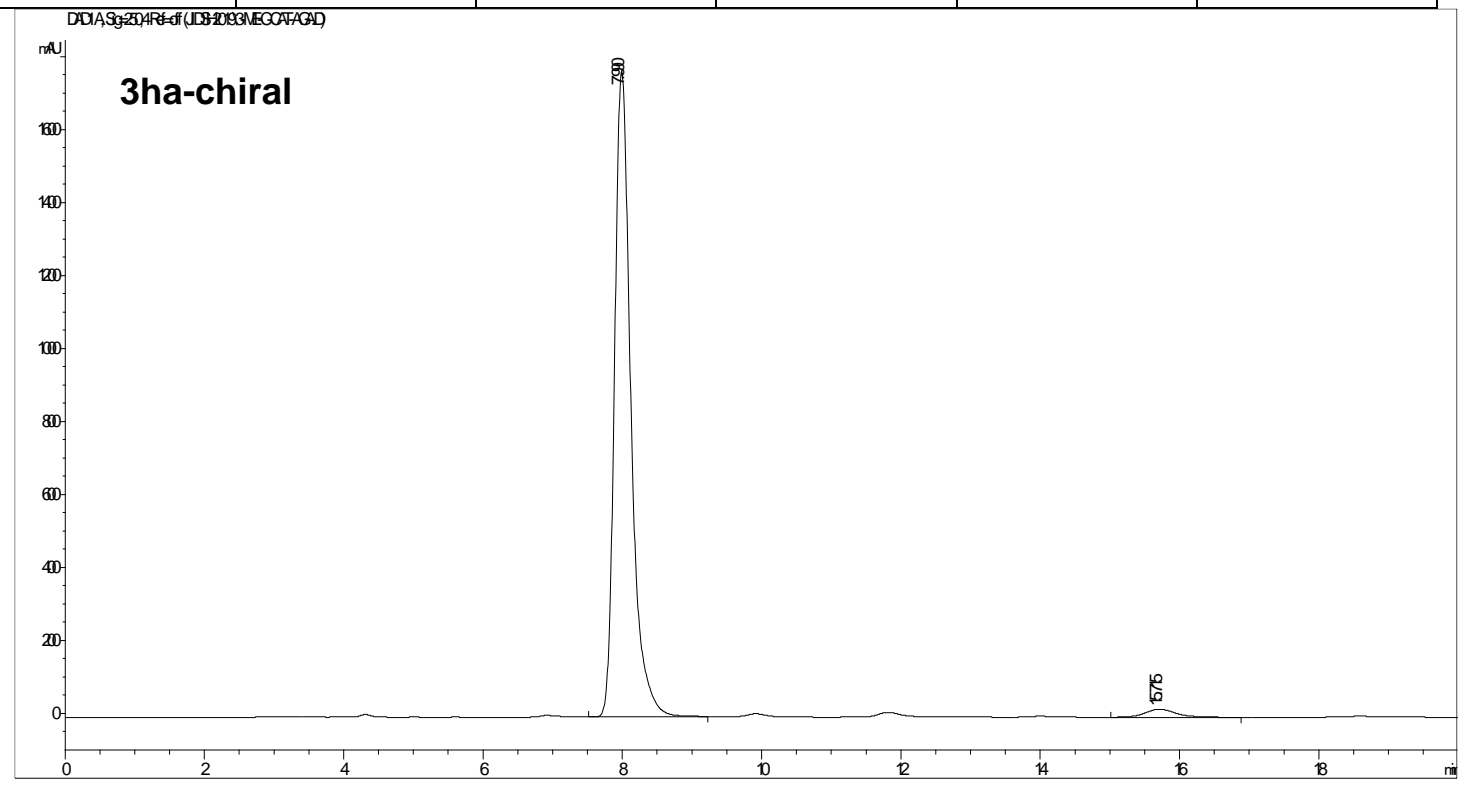

\begin{tabular}{|c|c|l|c|c|c|}
\hline Peak & $\begin{array}{c}\text { Processed } \\
\text { Channel }\end{array}$ & $\begin{array}{c}\text { Retention } \\
\text { Time (min) }\end{array}$ & $\begin{array}{c}\text { Peak Area } \\
(\mathrm{mAU} * \mathrm{~s})\end{array}$ & $\begin{array}{c}\text { Peak } \\
\text { Height } \\
(\mathrm{mAU})\end{array}$ & $\begin{array}{c}\text { Peak Area } \\
(\%)\end{array}$ \\
\hline 1 & $\begin{array}{c}\text { DAD 254, } \\
4 \mathrm{~nm}\end{array}$ & 7.99 & 28753.7 & 1769.9 & 97.71 \\
\hline 2 & $\begin{array}{c}\text { DAD 254, } \\
4 \mathrm{~nm}\end{array}$ & 15.72 & 672.8 & 22 & 2.29 \\
\hline
\end{tabular}




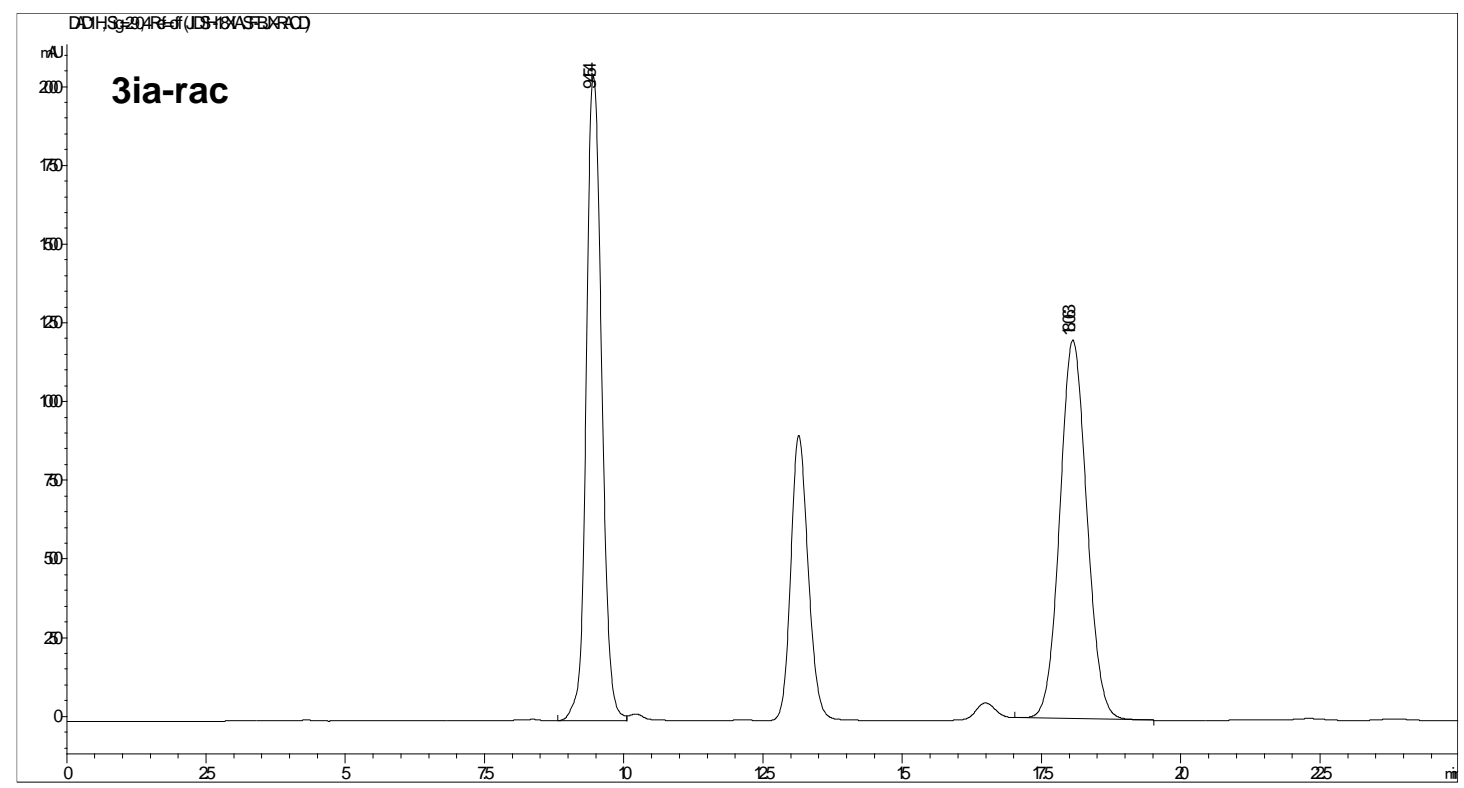

\begin{tabular}{|c|c|c|c|c|c|}
\hline Peak & $\begin{array}{c}\text { Processed } \\
\text { Channel }\end{array}$ & $\begin{array}{c}\text { Retention } \\
\text { Time (min) }\end{array}$ & $\begin{array}{c}\text { Peak Area } \\
\left.(\mathrm{mAU})^{*}\right)\end{array}$ & $\begin{array}{c}\text { Peak } \\
\text { Height } \\
(\mathrm{mAU})\end{array}$ & $\begin{array}{c}\text { Peak Area } \\
(\%)\end{array}$ \\
\hline 1 & $\begin{array}{c}\text { DAD 254, } \\
4 \mathrm{~nm}\end{array}$ & 10.463 & 102265.6 & 3774.8 & 49.9851 \\
\hline 2 & $\begin{array}{c}\text { DAD 254, } \\
4 \mathrm{~nm}\end{array}$ & 21.273 & 3526.5 & 88.4 & 50.0149 \\
\hline
\end{tabular}

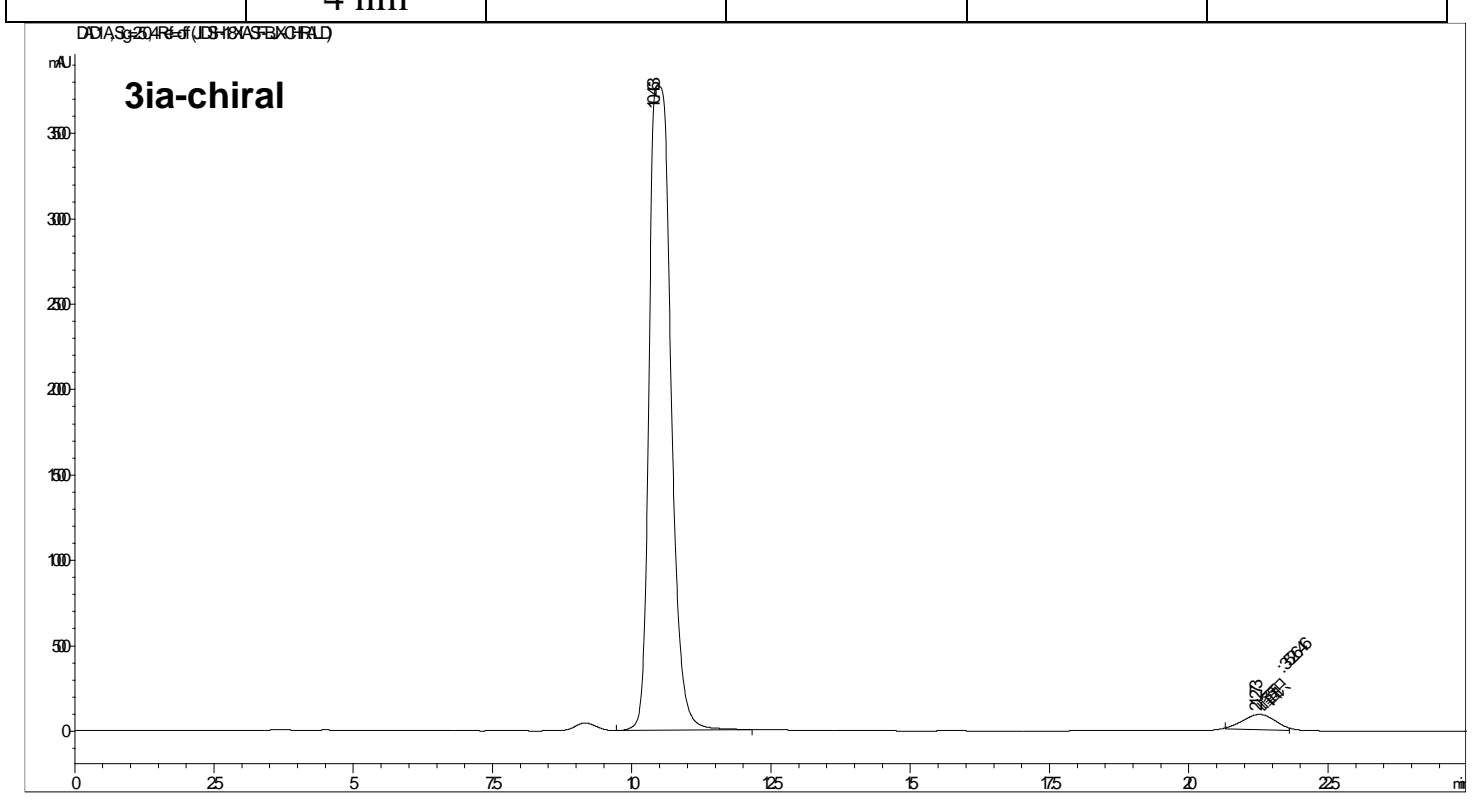

\begin{tabular}{|c|c|c|c|c|c|}
\hline Peak & Processed & Peak & Processed & Peak & Processed \\
\hline 1 & $\begin{array}{c}\text { DAD 254, } \\
4 \mathrm{~nm}\end{array}$ & 10.463 & 102265.6 & 3774.8 & 97.667 \\
\hline 2 & $\begin{array}{c}\text { DAD 254, } \\
4 \mathrm{~nm}\end{array}$ & 21.273 & 3526.5 & 88.4 & 3.333 \\
\hline
\end{tabular}




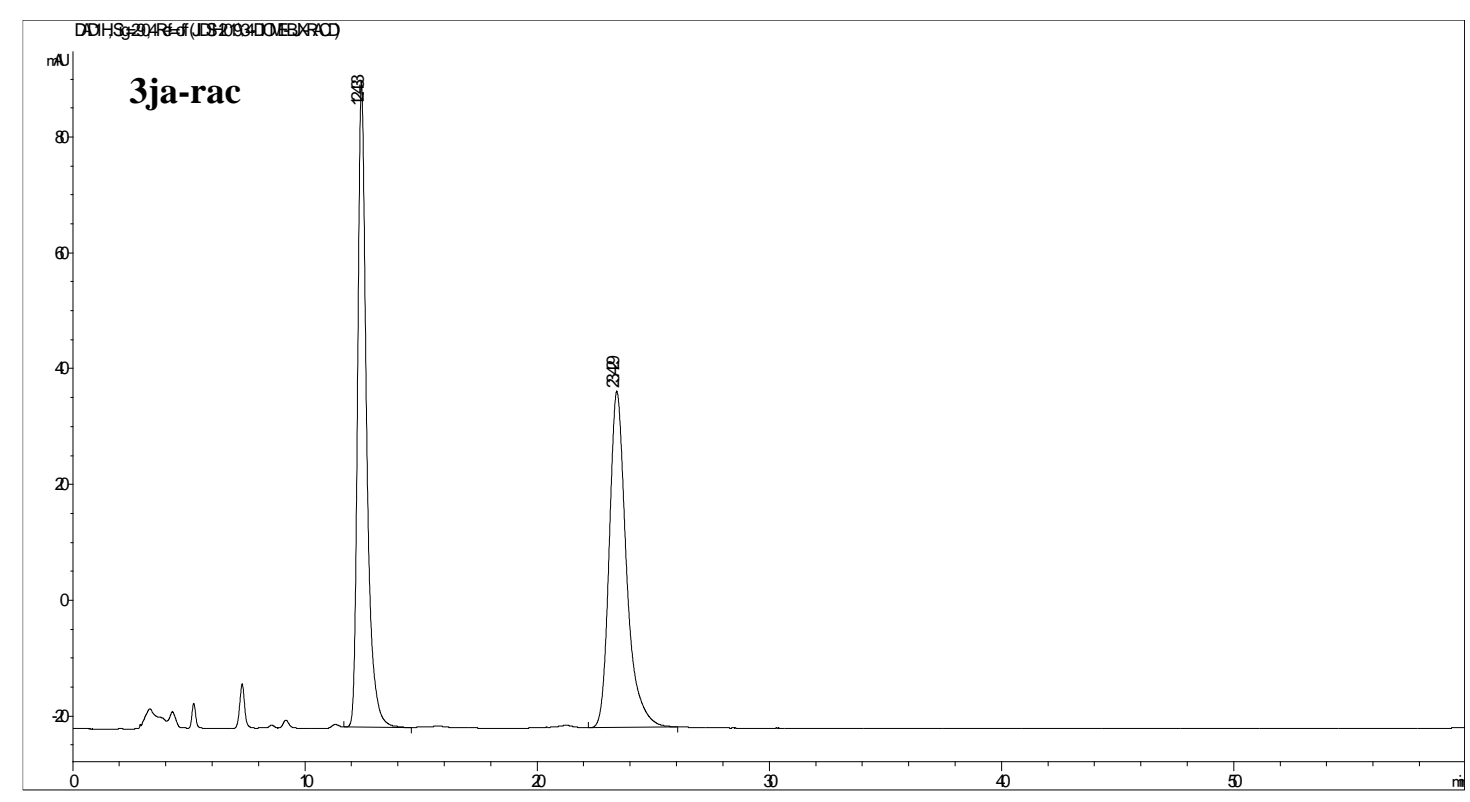

\begin{tabular}{|c|c|c|c|c|c|}
\hline Peak & $\begin{array}{c}\text { Processed } \\
\text { Channel }\end{array}$ & $\begin{array}{c}\text { Retention } \\
\text { Time (min) }\end{array}$ & $\begin{array}{c}\text { Peak Area } \\
\left(\mathrm{Mau}^{*} \mathrm{~s}\right)\end{array}$ & $\begin{array}{c}\text { Peak } \\
\text { Height } \\
(\mathrm{mAU})\end{array}$ & $\begin{array}{c}\text { Peak Area } \\
(\%)\end{array}$ \\
\hline 1 & $\begin{array}{c}\text { DAD 254, } \\
4 \mathrm{~nm}\end{array}$ & 12.433 & 2986.1 & 111.3 & 50.026 \\
\hline 2 & $\begin{array}{c}\text { DAD 254, } \\
4 \mathrm{~nm}\end{array}$ & 23.429 & 2983 & 58.1 & 49.974 \\
\hline
\end{tabular}

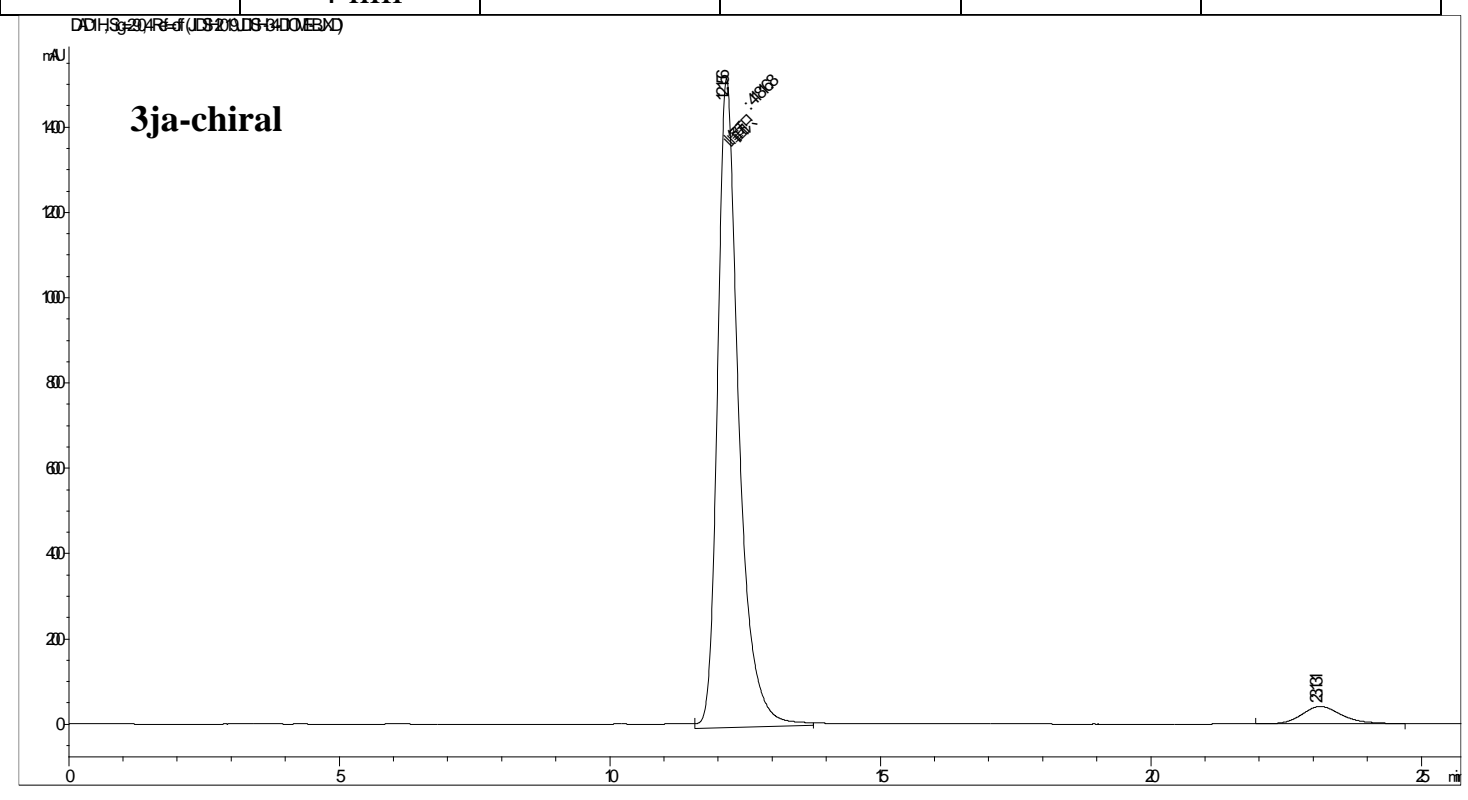

\begin{tabular}{|c|c|c|c|c|c|}
\hline Peak & $\begin{array}{c}\text { Processed } \\
\text { Channel }\end{array}$ & $\begin{array}{c}\text { Retention } \\
\text { Time (min) }\end{array}$ & $\begin{array}{c}\text { Peak Area } \\
\left(\mathrm{Mau}^{*} \mathrm{~s}\right)\end{array}$ & $\begin{array}{c}\text { Peak } \\
\text { Height } \\
(\mathrm{mAU})\end{array}$ & $\begin{array}{c}\text { Peak Area } \\
(\%)\end{array}$ \\
\hline 1 & $\begin{array}{c}\text { DAD 254, } \\
4 \mathrm{~nm}\end{array}$ & 12.156 & 41816.8 & 1523.5 & 95.268 \\
\hline 2 & $\begin{array}{c}\text { DAD 254, } \\
4 \mathrm{~nm}\end{array}$ & 23.131 & 2076.9 & 40.8 & 4.732 \\
\hline
\end{tabular}




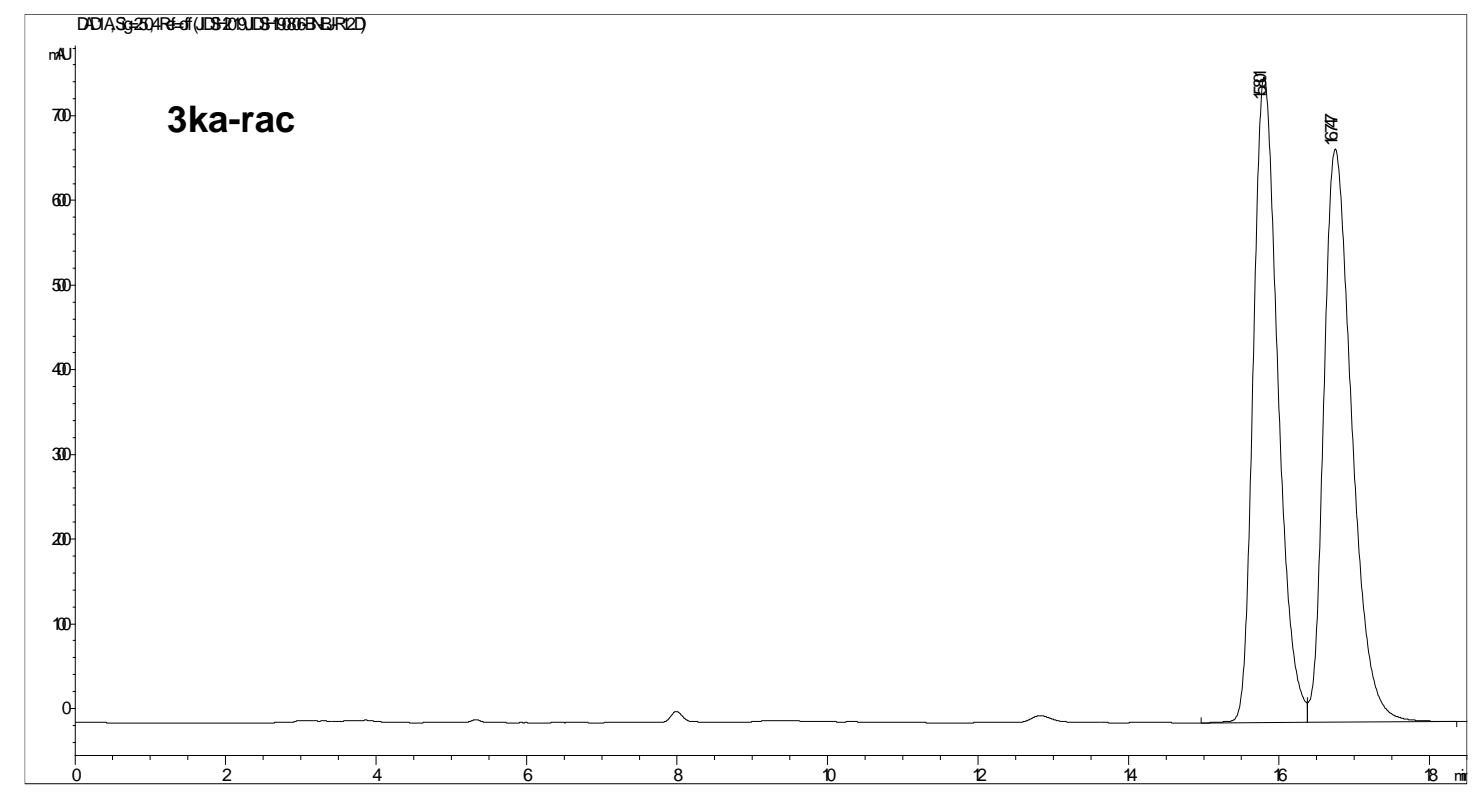

\begin{tabular}{|c|c|c|c|c|c|}
\hline Peak & $\begin{array}{c}\text { Processed } \\
\text { Channel }\end{array}$ & $\begin{array}{c}\text { Retention } \\
\text { Time (min) }\end{array}$ & $\begin{array}{c}\text { Peak Area } \\
(\mathrm{mAU} \text { * })\end{array}$ & $\begin{array}{c}\text { Peak } \\
\text { Height } \\
(\mathrm{mAU})\end{array}$ & $\begin{array}{c}\text { Peak Area } \\
(\%)\end{array}$ \\
\hline 1 & $\begin{array}{c}\text { DAD 254, } \\
4 \mathrm{~nm}\end{array}$ & 15.80 & 17359.1 & 762.3 & 49.77 \\
\hline 2 & $\begin{array}{c}\text { DAD 254, } \\
4 \mathrm{~nm}\end{array}$ & 16.75 & 17519.1 & 677 & 50.23 \\
\hline
\end{tabular}

${ }_{50}$ 3ka-chiral

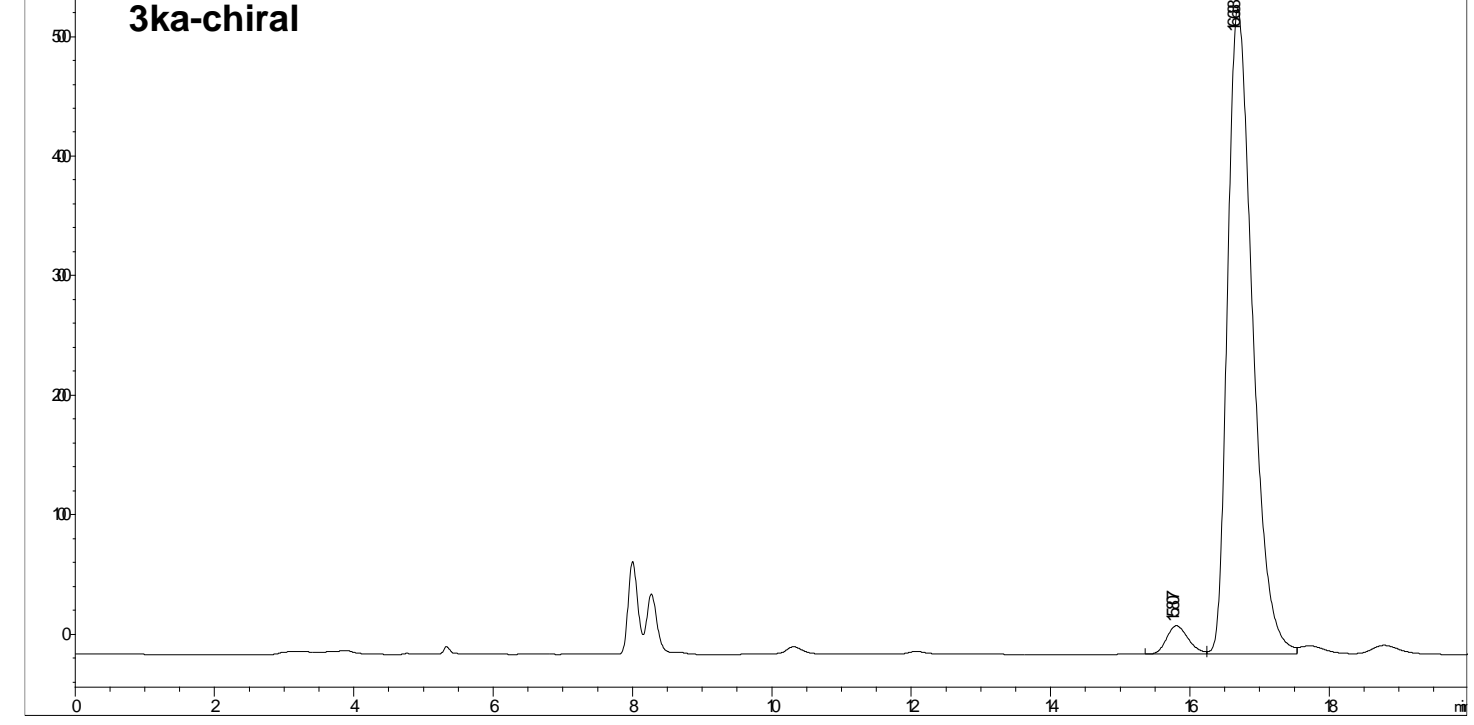

\begin{tabular}{|c|c|c|c|c|c|}
\hline Peak & $\begin{array}{c}\text { Processed } \\
\text { Channel }\end{array}$ & $\begin{array}{c}\text { Retention } \\
\text { Time (min) }\end{array}$ & $\begin{array}{c}\text { Peak Area } \\
\left(\mathrm{mAU}^{*} \mathrm{~s}\right)\end{array}$ & $\begin{array}{c}\text { Peak } \\
\text { Height } \\
(\mathrm{mAU})\end{array}$ & $\begin{array}{c}\text { Peak Area } \\
(\%)\end{array}$ \\
\hline 1 & $\begin{array}{c}\text { DAD 254, } \\
4 \mathrm{~nm}\end{array}$ & 15.81 & 511.7 & 23.7 & 3.67 \\
\hline 2 & $\begin{array}{c}\text { DAD 254, } \\
4 \mathrm{~nm}\end{array}$ & 16.67 & 13446.3 & 539.7 & 96.33 \\
\hline
\end{tabular}




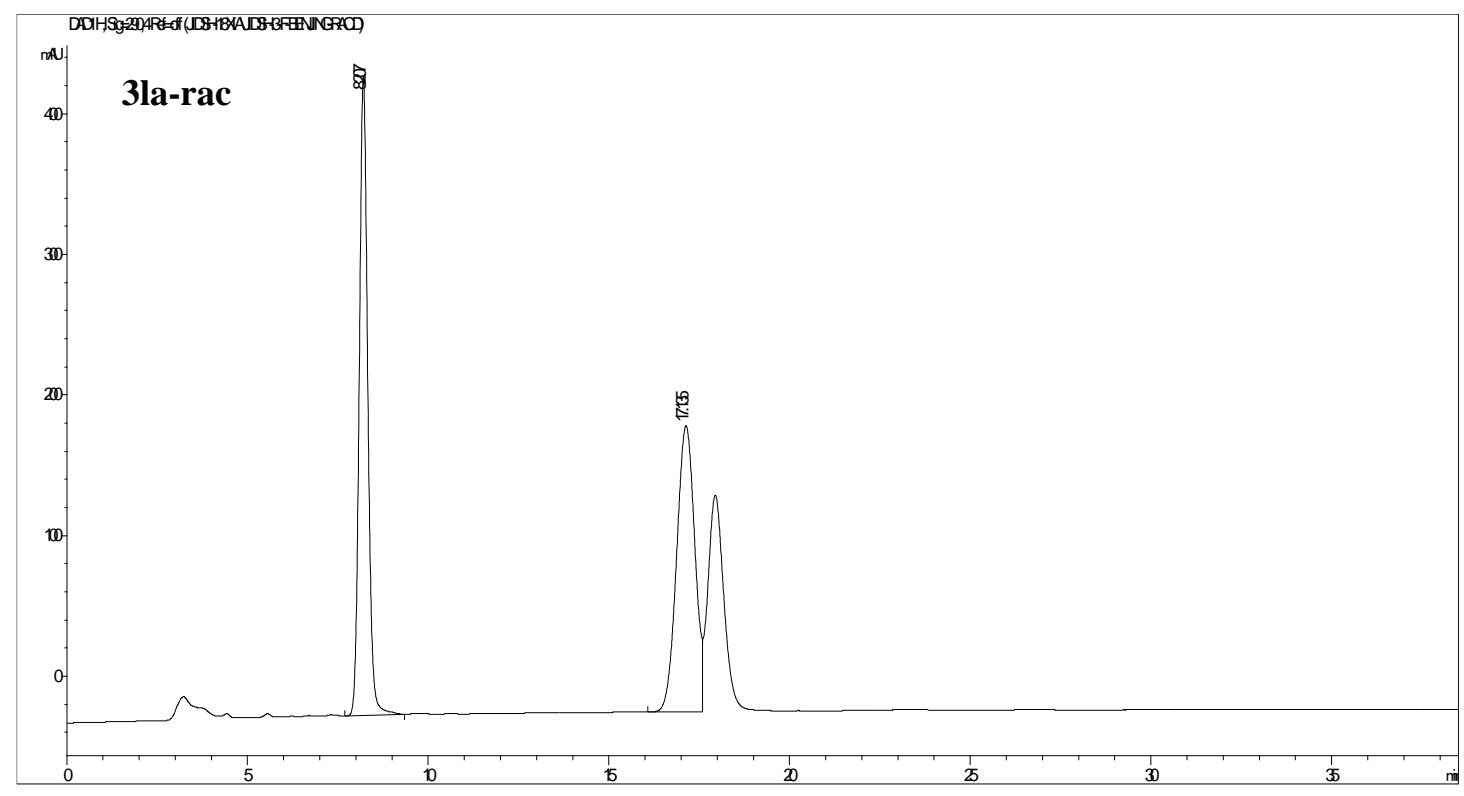

\begin{tabular}{|c|c|c|c|c|c|}
\hline Peak & $\begin{array}{c}\text { Processed } \\
\text { Channel }\end{array}$ & $\begin{array}{c}\text { Retention } \\
\text { Time (min) }\end{array}$ & $\begin{array}{c}\text { Peak Area } \\
(\mathrm{mAU} \text { *s })\end{array}$ & $\begin{array}{c}\text { Peak } \\
\text { Height } \\
(\mathrm{mAU})\end{array}$ & $\begin{array}{c}\text { Peak Area } \\
(\%)\end{array}$ \\
\hline 1 & $\begin{array}{c}\text { DAD 254, } \\
4 \mathrm{~nm}\end{array}$ & 8.207 & 7432.4 & 454.1 & 51.085 \\
\hline 2 & $\begin{array}{c}\text { DAD 254, } \\
4 \mathrm{~nm}\end{array}$ & 17.135 & 7116.6 & 203.3 & 49.915 \\
\hline
\end{tabular}

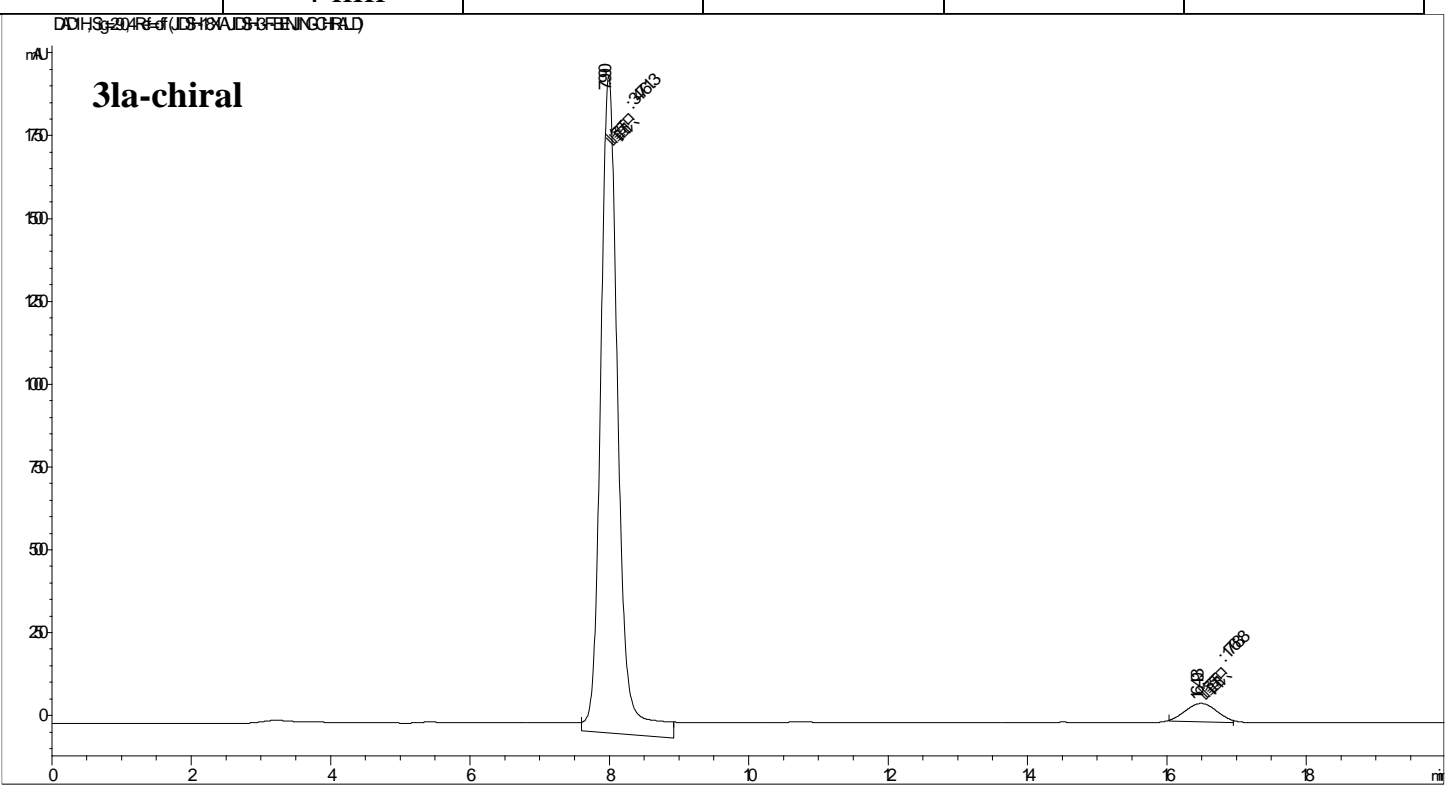

\begin{tabular}{|c|c|c|c|c|c|}
\hline Peak & $\begin{array}{c}\text { Processed } \\
\text { Channel }\end{array}$ & $\begin{array}{c}\text { Retention } \\
\text { Time (min) }\end{array}$ & $\begin{array}{c}\text { Peak Area } \\
(\text { Mau*s })\end{array}$ & $\begin{array}{c}\text { Peak } \\
\text { Height } \\
(\mathrm{mAU})\end{array}$ & $\begin{array}{c}\text { Peak Area } \\
(\%)\end{array}$ \\
\hline 1 & $\begin{array}{c}\text { DAD 254, } \\
4 \mathrm{~nm}\end{array}$ & 7.99 & 34761.3 & 1980.2 & 95.158 \\
\hline 2 & $\begin{array}{c}\text { DAD 254, } \\
4 \mathrm{~nm}\end{array}$ & 16.493 & 1768.8 & 56 & 4.842 \\
\hline
\end{tabular}




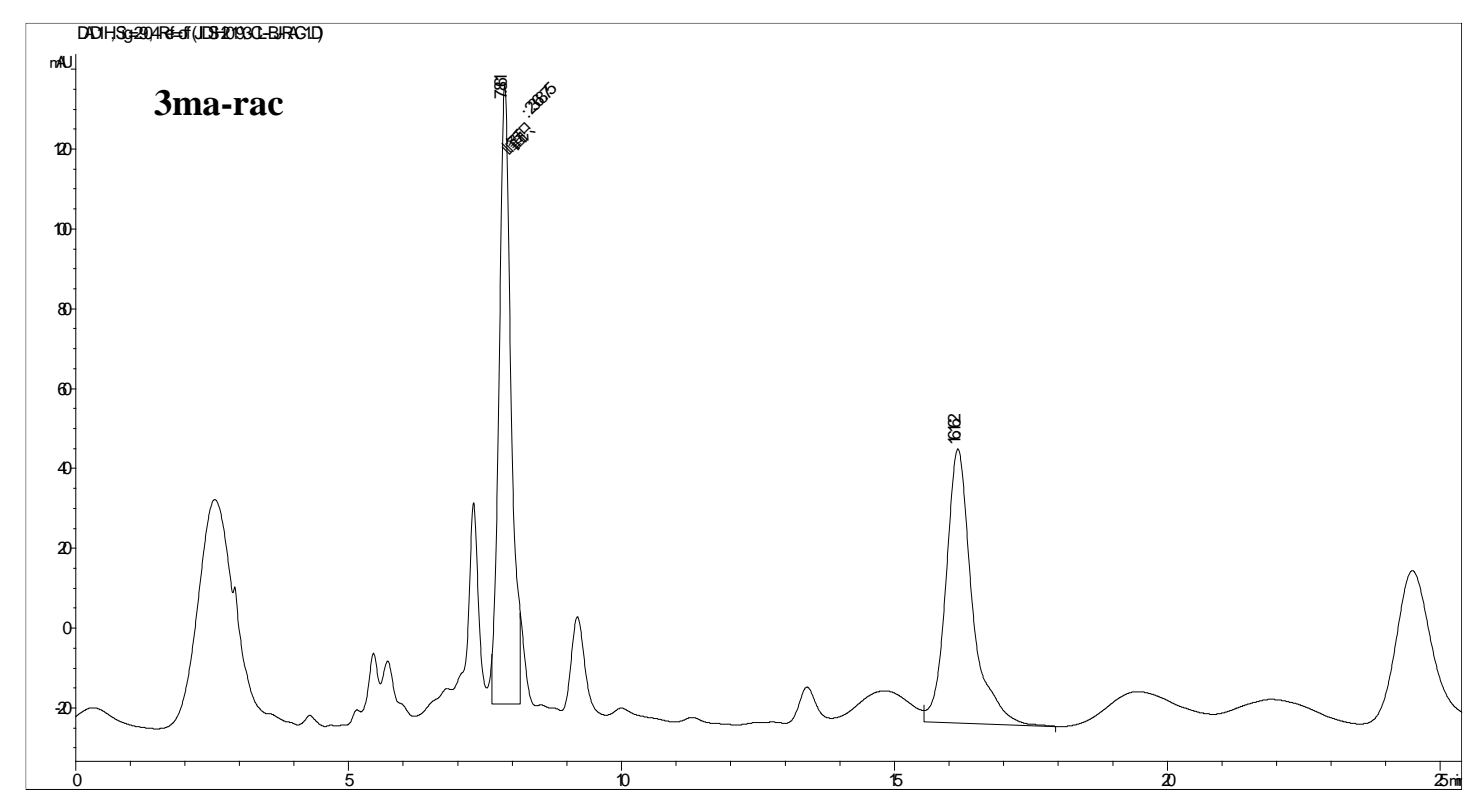

\begin{tabular}{|c|c|c|c|c|c|}
\hline Peak & $\begin{array}{c}\text { Processed } \\
\text { Channel }\end{array}$ & $\begin{array}{c}\text { Retention } \\
\text { Time (min) }\end{array}$ & $\begin{array}{c}\text { Peak Area } \\
(\mathrm{Mau} \text { *s) }\end{array}$ & $\begin{array}{c}\text { Peak } \\
\text { Height } \\
(\mathrm{mAU})\end{array}$ & $\begin{array}{c}\text { Peak Area } \\
(\%)\end{array}$ \\
\hline 1 & $\begin{array}{c}\text { DAD 254, } \\
4 \mathrm{~nm}\end{array}$ & 7.861 & 2368.8 & 154.9 & 51.022 \\
\hline 2 & $\begin{array}{c}\text { DAD 254, } \\
4 \mathrm{~nm}\end{array}$ & 16.162 & 2273.9 & 68.6 & 48.978 \\
\hline
\end{tabular}

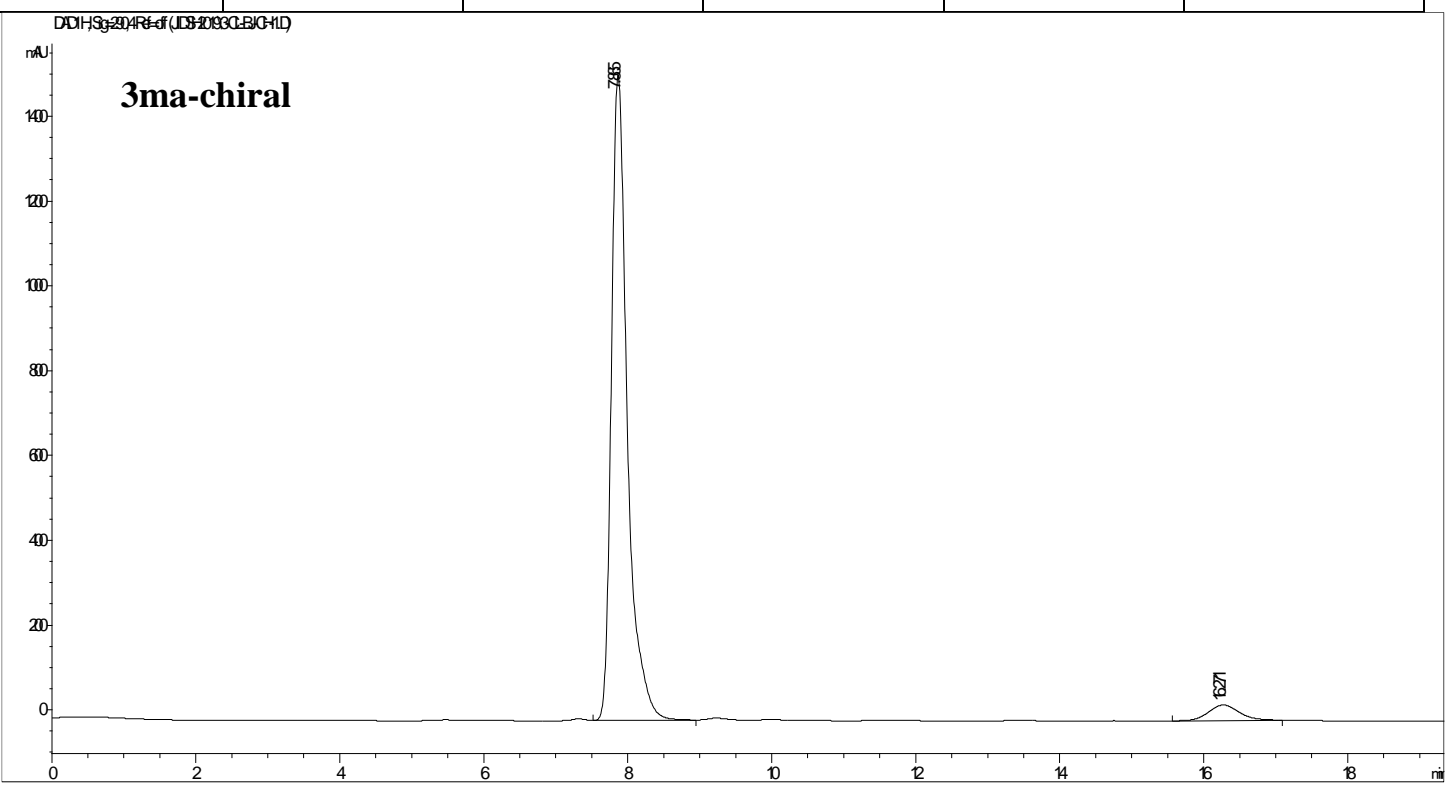

\begin{tabular}{|c|c|c|c|c|c|}
\hline Peak & $\begin{array}{c}\text { Processed } \\
\text { Channel }\end{array}$ & $\begin{array}{c}\text { Retention } \\
\text { Time (min) }\end{array}$ & $\begin{array}{c}\text { Peak Area } \\
\left(\mathrm{Mau}^{*} \mathrm{~s}\right)\end{array}$ & $\begin{array}{c}\text { Peak } \\
\text { Height } \\
(\mathrm{mAU})\end{array}$ & $\begin{array}{c}\text { Peak Area } \\
(\%)\end{array}$ \\
\hline 1 & $\begin{array}{c}\text { DAD 254, } \\
4 \mathrm{~nm}\end{array}$ & 7.865 & 23371 & 1522.2 & 95.594 \\
\hline 2 & $\begin{array}{c}\text { DAD 254, } \\
4 \mathrm{~nm}\end{array}$ & 16.271 & 1077.1 & 36.4 & 4.406 \\
\hline
\end{tabular}




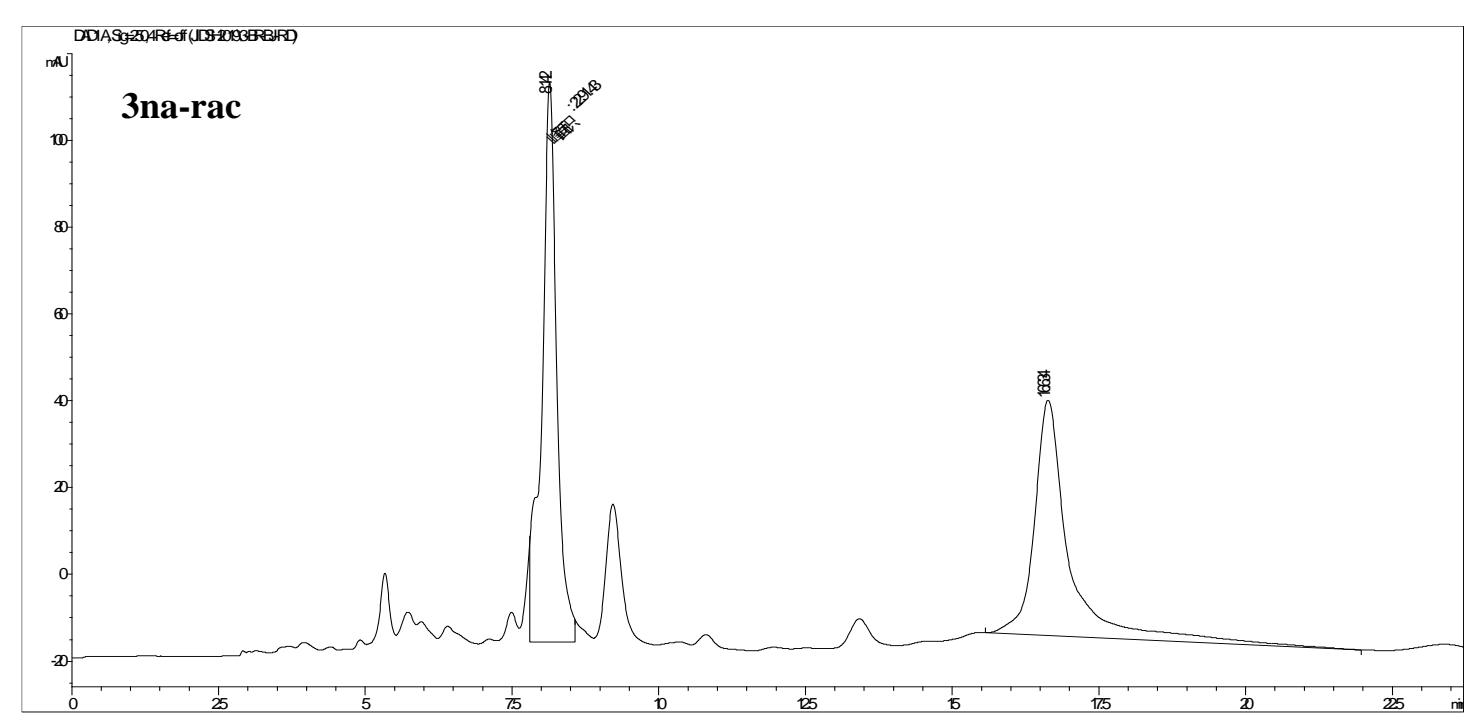

\begin{tabular}{|c|c|c|c|c|c|}
\hline Peak & $\begin{array}{c}\text { Processed } \\
\text { Channel }\end{array}$ & $\begin{array}{c}\text { Retention } \\
\text { Time (min) }\end{array}$ & $\begin{array}{c}\text { Peak Area } \\
(\text { Mau*s })\end{array}$ & $\begin{array}{c}\text { Peak } \\
\text { Height } \\
(\mathrm{mAU})\end{array}$ & $\begin{array}{c}\text { Peak Area } \\
(\%)\end{array}$ \\
\hline 1 & $\begin{array}{c}\text { DAD 254, } \\
4 \mathrm{~nm}\end{array}$ & 8.142 & 2291.4 & 129.1 & 50.328 \\
\hline 2 & $\begin{array}{c}\text { DAD 254, } \\
4 \mathrm{~nm}\end{array}$ & 16.634 & 2261.6 & 54.2 & 49.672 \\
\hline
\end{tabular}

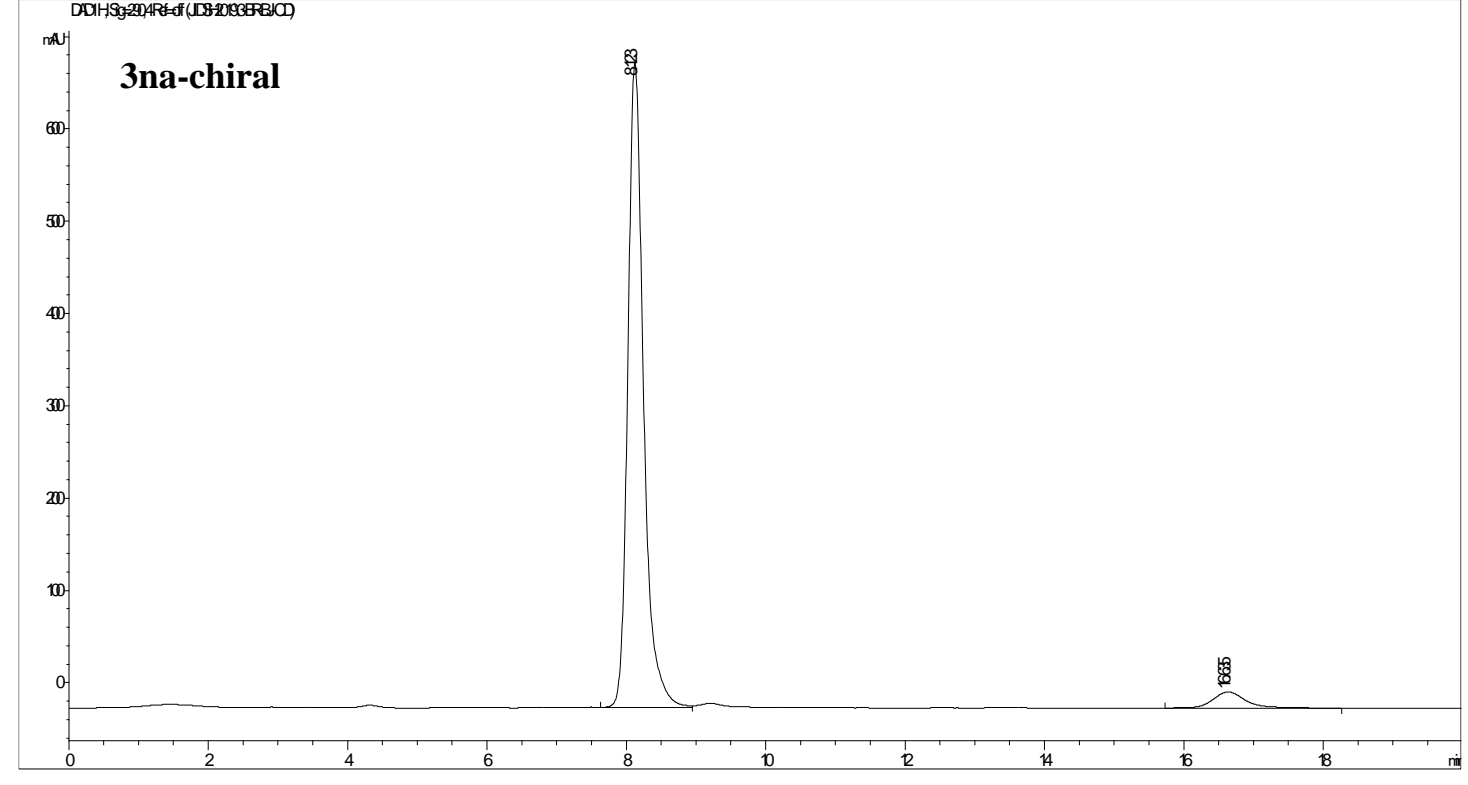

\begin{tabular}{|c|c|c|c|c|c|}
\hline Peak & $\begin{array}{c}\text { Processed } \\
\text { Channel }\end{array}$ & $\begin{array}{c}\text { Retention } \\
\text { Time (min) }\end{array}$ & $\begin{array}{c}\text { Peak Area } \\
(\text { Mau*s })\end{array}$ & $\begin{array}{c}\text { Peak } \\
\text { Height } \\
(\mathrm{mAU})\end{array}$ & $\begin{array}{c}\text { Peak Area } \\
(\%)\end{array}$ \\
\hline 1 & $\begin{array}{c}\text { DAD 254, } \\
4 \mathrm{~nm}\end{array}$ & 8.123 & 10927.2 & 699.2 & 95.112 \\
\hline 2 & $\begin{array}{c}\text { DAD 254, } \\
4 \mathrm{~nm}\end{array}$ & 16.635 & 561.6 & 17.3 & 4.888 \\
\hline
\end{tabular}




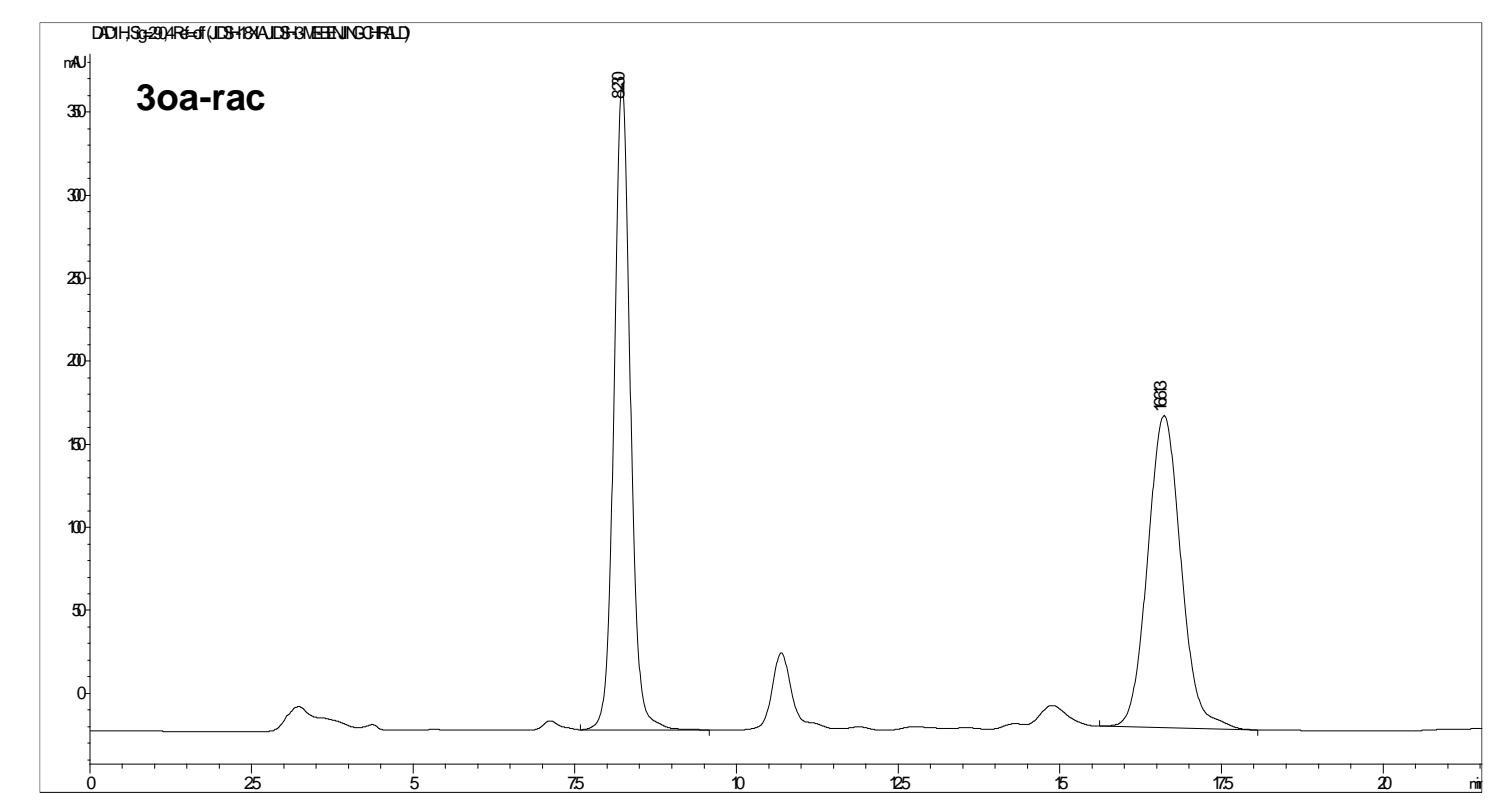

\begin{tabular}{|c|c|c|c|c|c|}
\hline Peak & $\begin{array}{c}\text { Processed } \\
\text { Channel }\end{array}$ & $\begin{array}{c}\text { Retention } \\
\text { Time (min) }\end{array}$ & $\begin{array}{c}\text { Peak Area } \\
\left(\mathrm{Mau}^{*} \mathrm{~s}\right)\end{array}$ & $\begin{array}{c}\text { Peak } \\
\text { Height } \\
(\mathrm{mAU})\end{array}$ & $\begin{array}{c}\text { Peak Area } \\
(\%)\end{array}$ \\
\hline 1 & $\begin{array}{c}\text { DAD 254, } \\
4 \mathrm{~nm}\end{array}$ & 8.23 & 6861 & 388.1 & 50.383 \\
\hline 2 & $\begin{array}{c}\text { DAD 254, } \\
4 \mathrm{~nm}\end{array}$ & 16.613 & 6756.6 & 188 & 49.617 \\
\hline
\end{tabular}

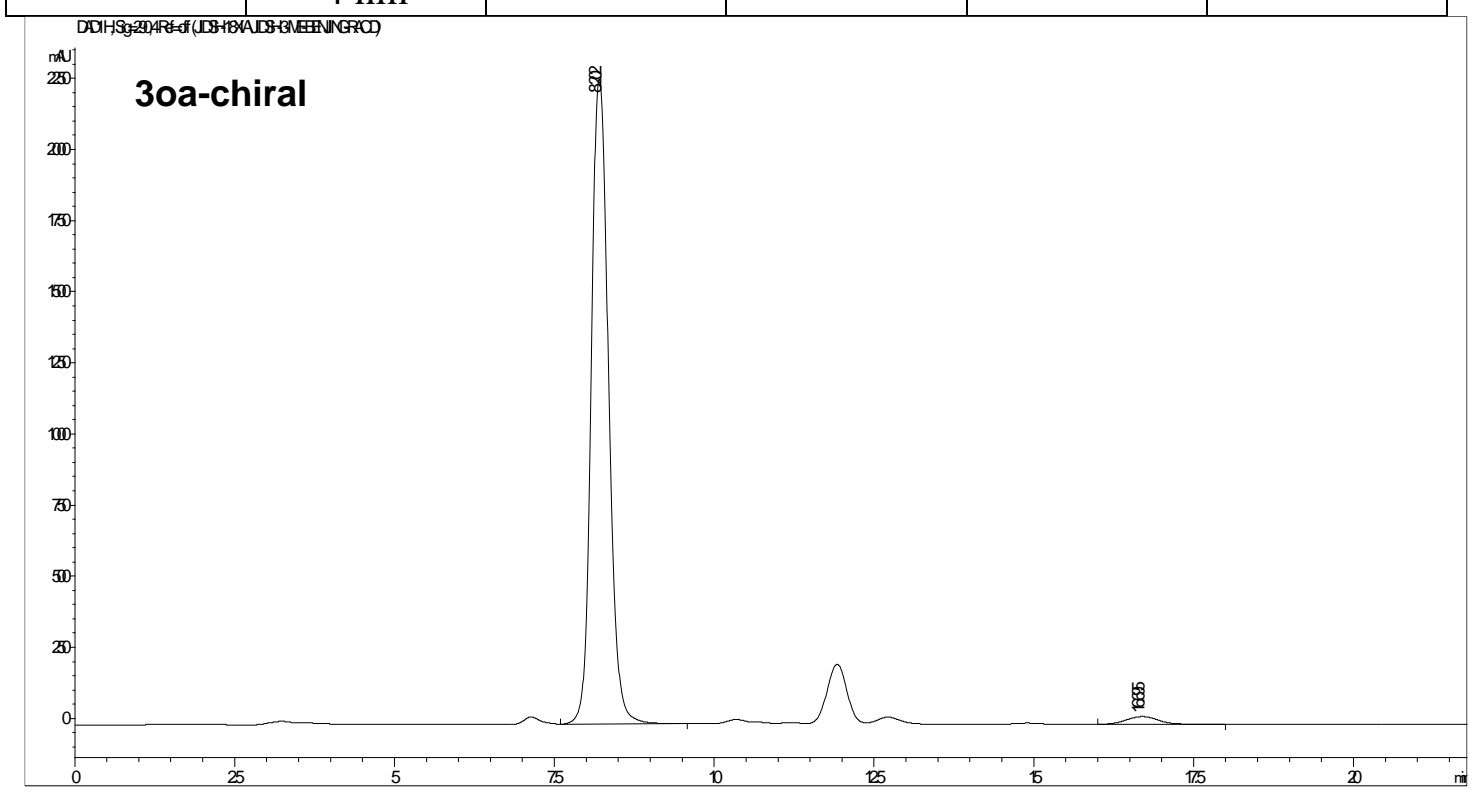

\begin{tabular}{|c|c|c|c|c|c|}
\hline Peak & $\begin{array}{c}\text { Processed } \\
\text { Channel }\end{array}$ & $\begin{array}{c}\text { Retention } \\
\text { Time (min) }\end{array}$ & $\begin{array}{c}\text { Peak Area } \\
\left(\mathrm{Mau}^{*} \mathrm{~s}\right)\end{array}$ & $\begin{array}{c}\text { Peak } \\
\text { Height } \\
(\mathrm{mAU})\end{array}$ & $\begin{array}{c}\text { Peak Area } \\
(\%)\end{array}$ \\
\hline 1 & $\begin{array}{c}\text { DAD 254, } \\
4 \mathrm{~nm}\end{array}$ & 8.202 & 42689.8 & 2267.7 & 97.747 \\
\hline 2 & $\begin{array}{c}\text { DAD 254, } \\
4 \mathrm{~nm}\end{array}$ & 16.695 & 984.1 & 27.5 & 2.253 \\
\hline
\end{tabular}




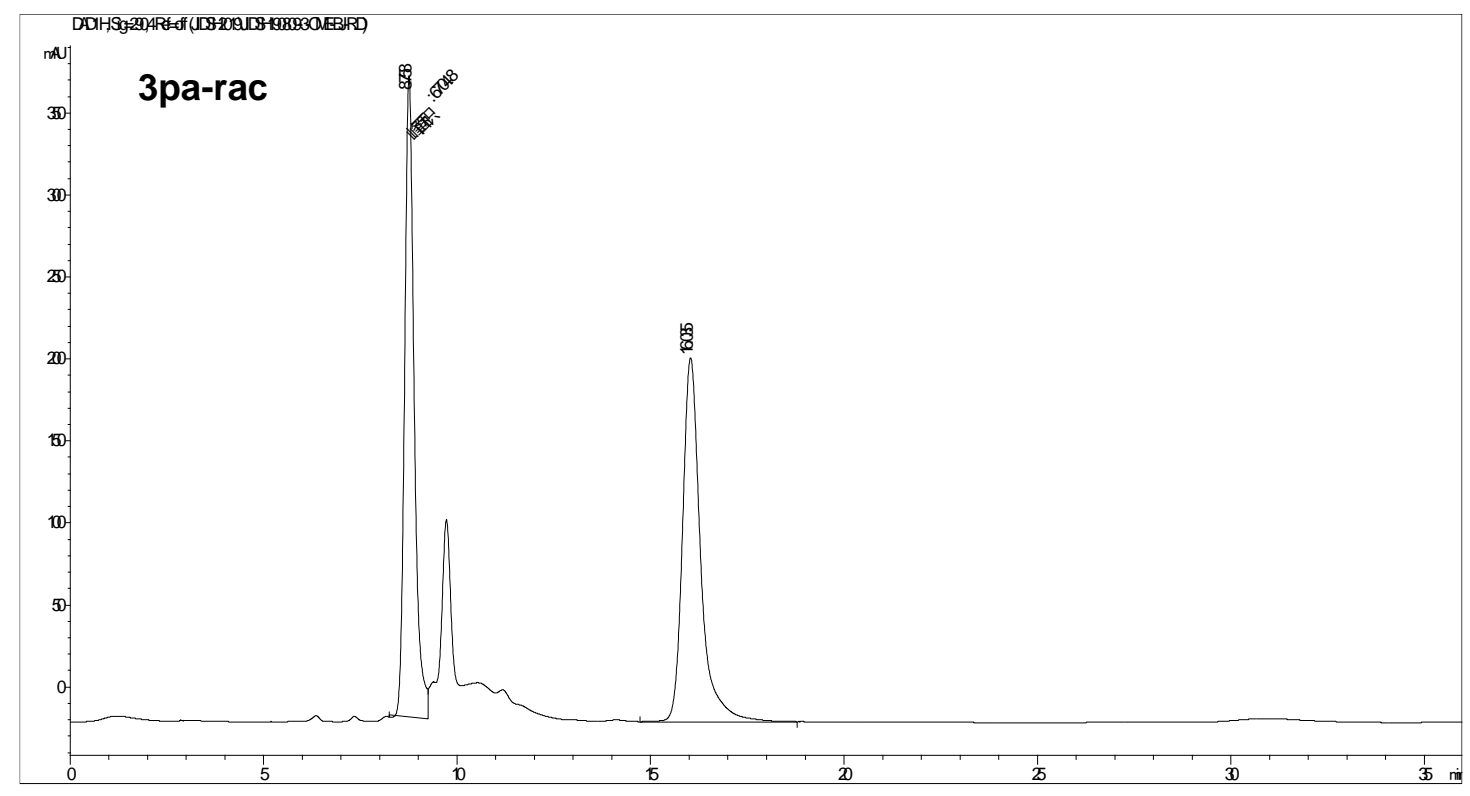

\begin{tabular}{|c|c|c|c|c|c|}
\hline Peak & $\begin{array}{c}\text { Processed } \\
\text { Channel }\end{array}$ & $\begin{array}{c}\text { Retention } \\
\text { Time (min) }\end{array}$ & $\begin{array}{c}\text { Peak Area } \\
\left(\mathrm{mAU}^{*} \mathrm{~s}\right)\end{array}$ & $\begin{array}{c}\text { Peak } \\
\text { Height } \\
(\mathrm{mAU})\end{array}$ & $\begin{array}{c}\text { Peak Area } \\
(\%)\end{array}$ \\
\hline 1 & $\begin{array}{c}\text { DAD 254, } \\
4 \mathrm{~nm}\end{array}$ & 8.758 & 6704.8 & 390.2 & 48.82 \\
\hline 2 & $\begin{array}{c}\text { DAD 254, } \\
4 \mathrm{~nm}\end{array}$ & 16.035 & 7194.6 & 222 & 51.18 \\
\hline
\end{tabular}

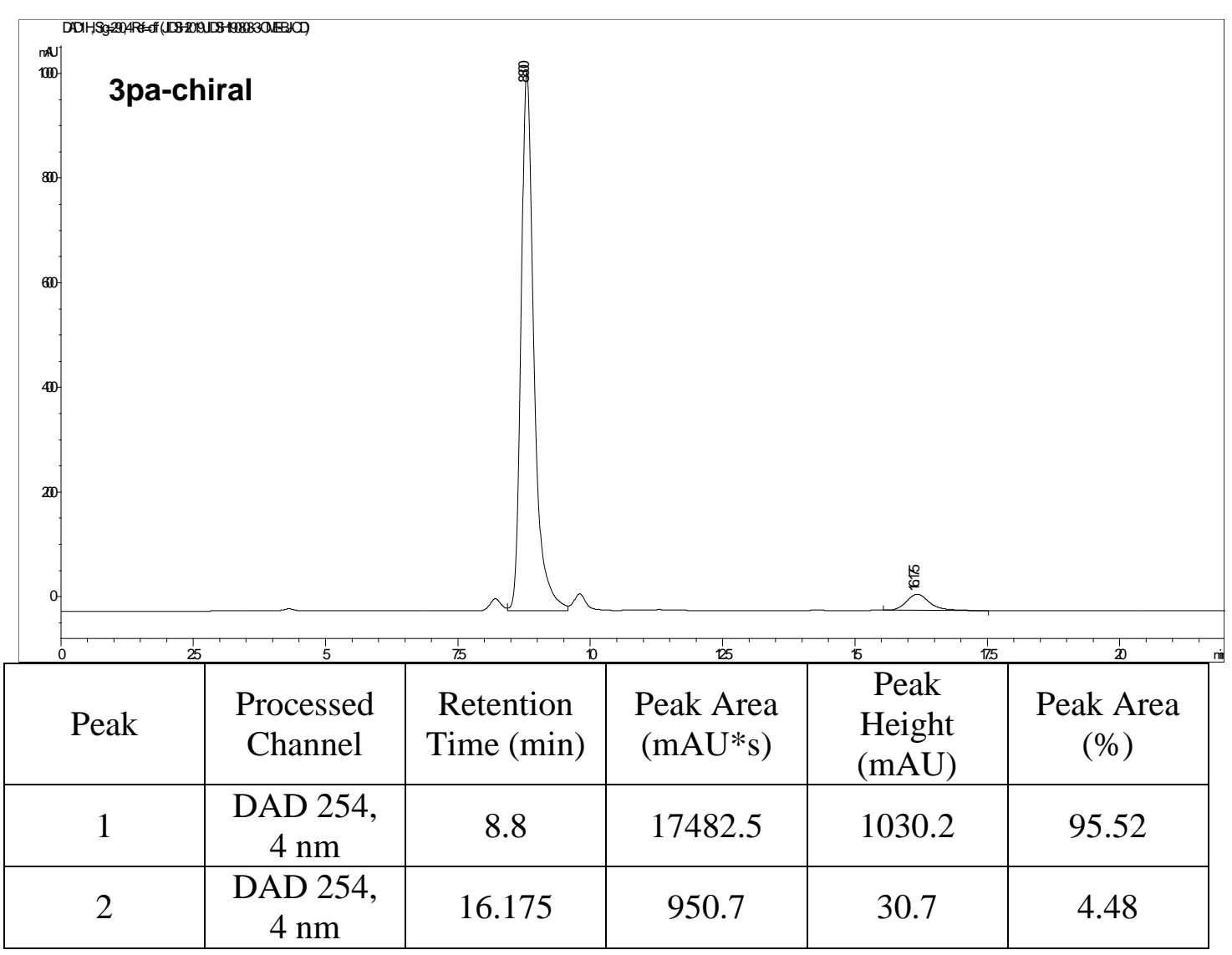




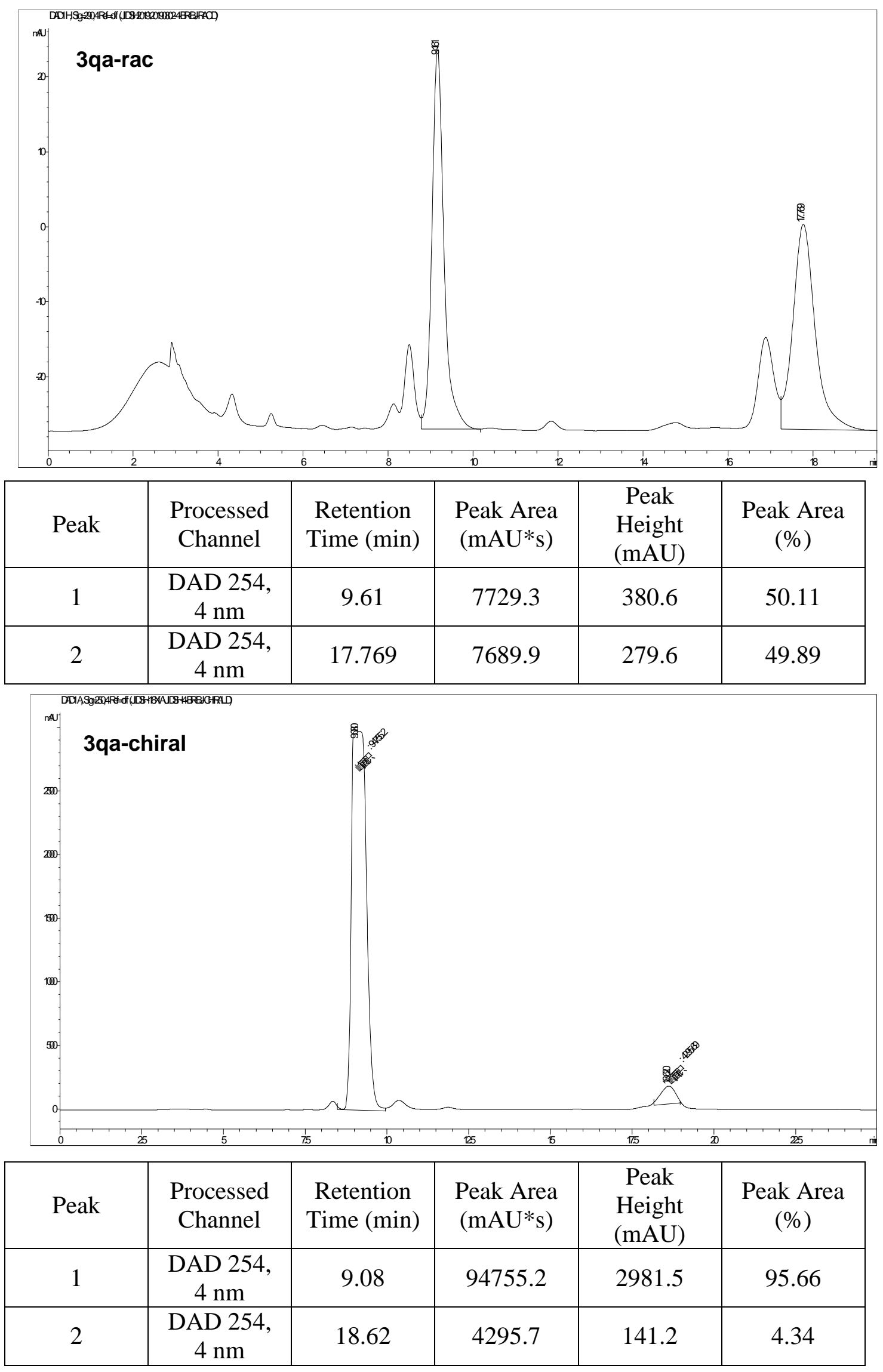




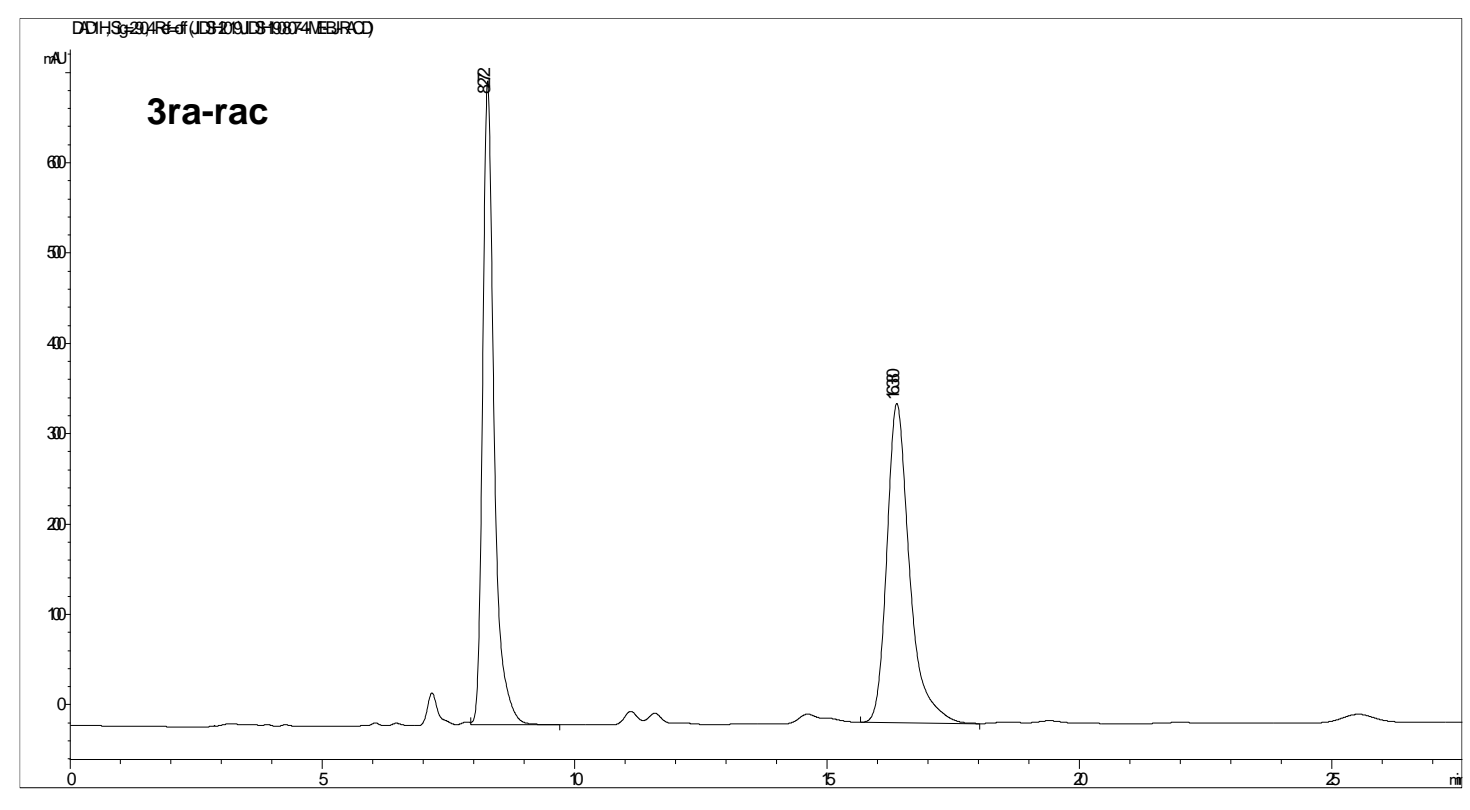

\begin{tabular}{|c|c|c|c|c|c|}
\hline Peak & $\begin{array}{c}\text { Processed } \\
\text { Channel }\end{array}$ & $\begin{array}{c}\text { Retention } \\
\text { Time (min) }\end{array}$ & $\begin{array}{c}\text { Peak Area } \\
(\mathrm{mAU} \text { ) })\end{array}$ & $\begin{array}{c}\text { Peak } \\
\text { Height } \\
(\mathrm{mAU})\end{array}$ & $\begin{array}{c}\text { Peak Area } \\
(\%)\end{array}$ \\
\hline 1 & $\begin{array}{c}\text { DAD 254, } \\
4 \mathrm{~nm}\end{array}$ & 8.273 & 17823.8 & 1128.3 & 50.17 \\
\hline 2 & $\begin{array}{c}\text { DAD 254, } \\
4 \mathrm{~nm}\end{array}$ & 16.38 & 17704.8 & 563 & 49.83 \\
\hline
\end{tabular}

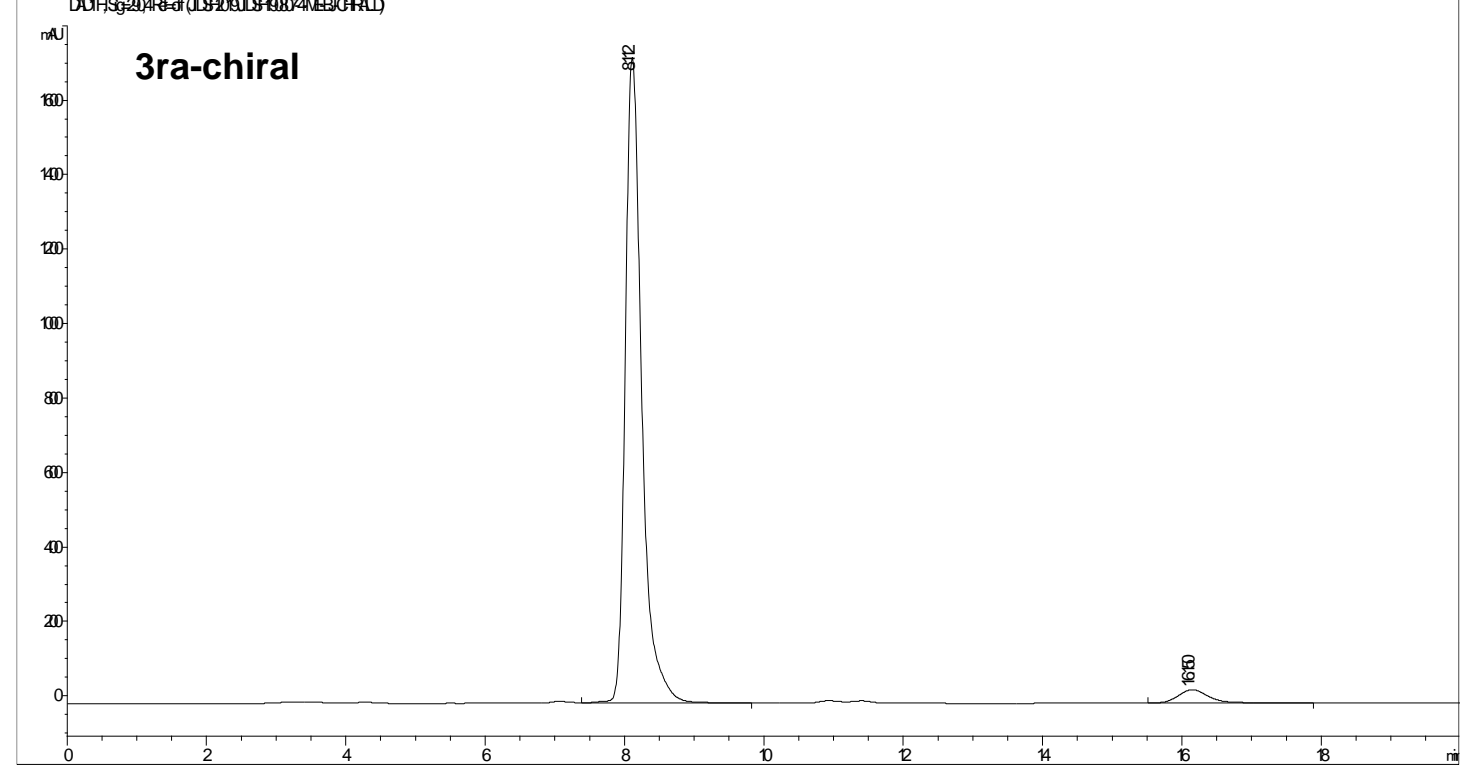

\begin{tabular}{|c|c|c|c|c|c|}
\hline Peak & $\begin{array}{c}\text { Processed } \\
\text { Channel }\end{array}$ & $\begin{array}{c}\text { Retention } \\
\text { Time (min) }\end{array}$ & $\begin{array}{c}\text { Peak Area } \\
\left(\mathrm{mAU}^{*} \mathrm{~s}\right)\end{array}$ & $\begin{array}{c}\text { Peak } \\
\text { Height } \\
(\mathrm{mAU})\end{array}$ & $\begin{array}{c}\text { Peak Area } \\
(\%)\end{array}$ \\
\hline 1 & $\begin{array}{c}\text { DAD 254, } \\
4 \mathrm{~nm}\end{array}$ & 8.112 & 28401.9 & 1732.6 & 96.17 \\
\hline 2 & $\begin{array}{c}\text { DAD 254, } \\
4 \mathrm{~nm}\end{array}$ & 16.15 & 1131.4 & 36.1 & 3.83 \\
\hline
\end{tabular}




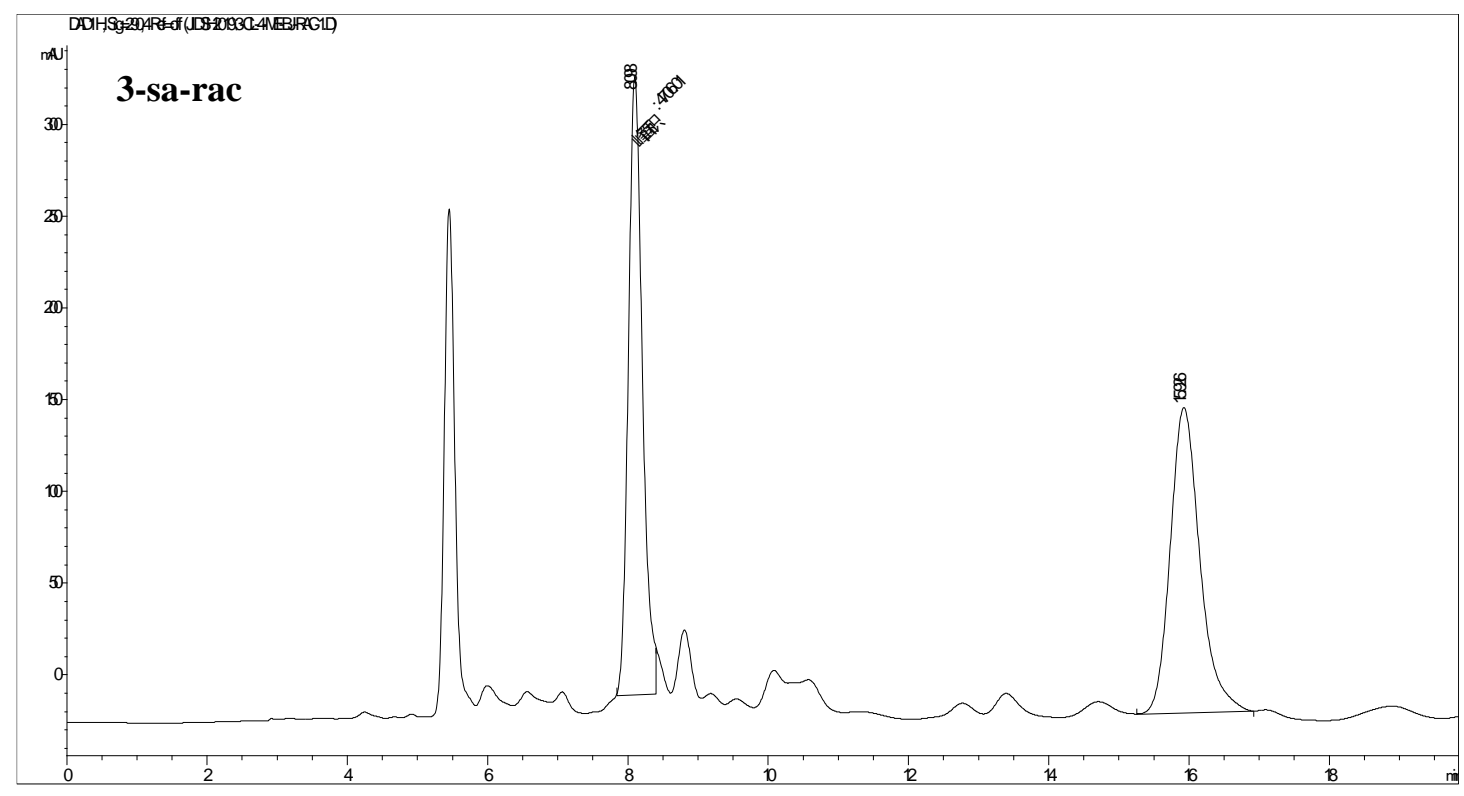

\begin{tabular}{|c|c|c|c|c|c|}
\hline Peak & $\begin{array}{c}\text { Processed } \\
\text { Channel }\end{array}$ & $\begin{array}{c}\text { Retention } \\
\text { Time (min) }\end{array}$ & $\begin{array}{c}\text { Peak Area } \\
(\text { Mau*s })\end{array}$ & $\begin{array}{c}\text { Peak } \\
\text { Height } \\
(\mathrm{mAU})\end{array}$ & $\begin{array}{c}\text { Peak Area } \\
(\%)\end{array}$ \\
\hline 1 & $\begin{array}{c}\text { DAD 254, } \\
4 \mathrm{~nm}\end{array}$ & 8.098 & 4706 & 337 & 49.140 \\
\hline 2 & $\begin{array}{c}\text { DAD 254, } \\
4 \mathrm{~nm}\end{array}$ & 15.926 & 4870.7 & 166.4 & 50.086 \\
\hline
\end{tabular}

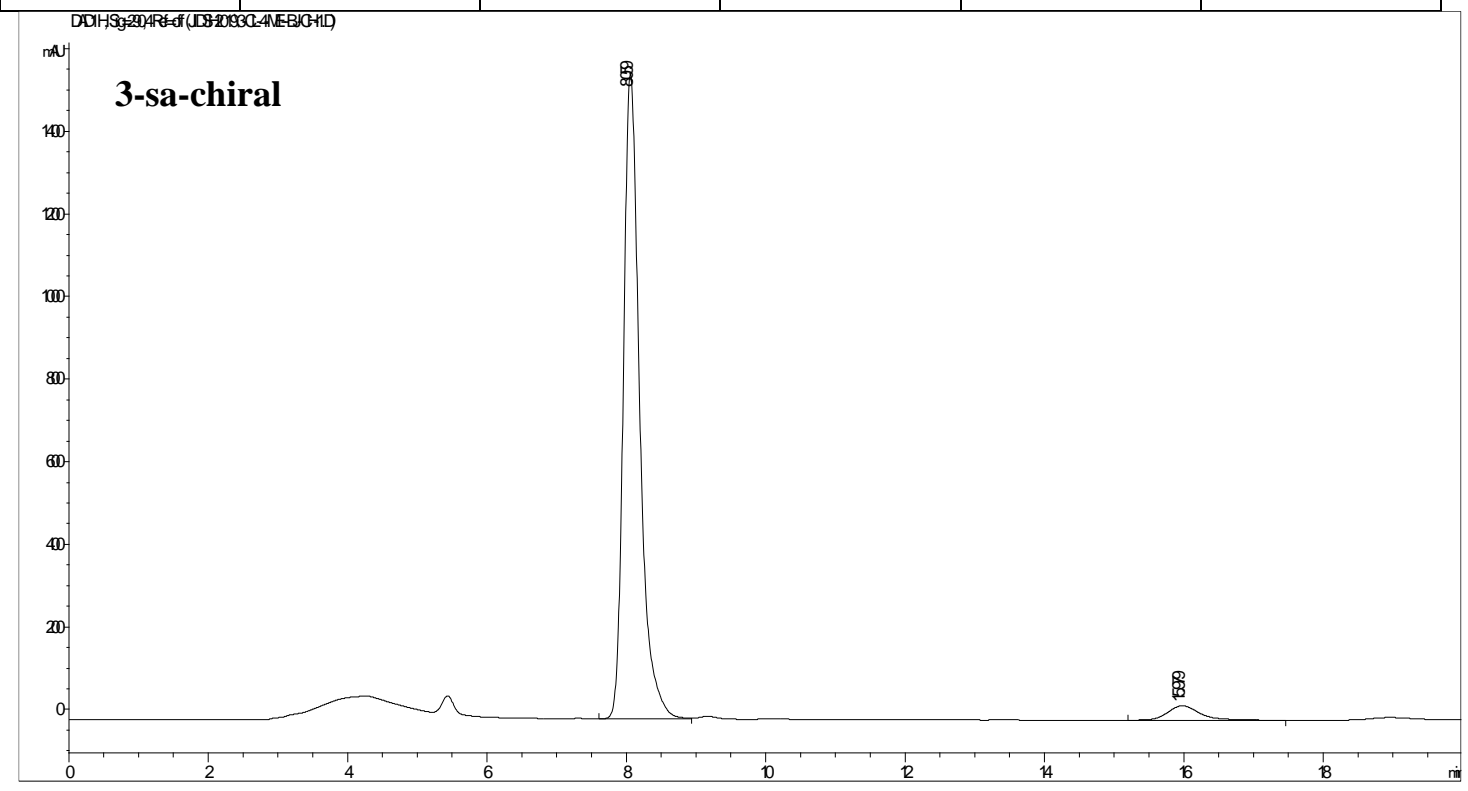

\begin{tabular}{|c|c|c|c|c|c|}
\hline Peak & $\begin{array}{c}\text { Processed } \\
\text { Channel }\end{array}$ & $\begin{array}{c}\text { Retention } \\
\text { Time (min) }\end{array}$ & $\begin{array}{c}\text { Peak Area } \\
(\text { Mau*s })\end{array}$ & $\begin{array}{c}\text { Peak } \\
\text { Height } \\
(\mathrm{mAU})\end{array}$ & $\begin{array}{c}\text { Peak Area } \\
(\%)\end{array}$ \\
\hline 1 & $\begin{array}{c}\text { DAD 254, } \\
4 \mathrm{~nm}\end{array}$ & 8.059 & 25170.9 & 1561.7 & 95.817 \\
\hline 2 & $\begin{array}{c}\text { DAD 254, } \\
4 \mathrm{~nm}\end{array}$ & 15.979 & 1098.7 & 34.7 & 4.183 \\
\hline
\end{tabular}

${ }^{1} \mathrm{H}-\mathrm{NMR}$ spectrum of $\mathbf{3 a a}$ in $\mathrm{CDCl}_{3}(400 \mathrm{MHz})$ 


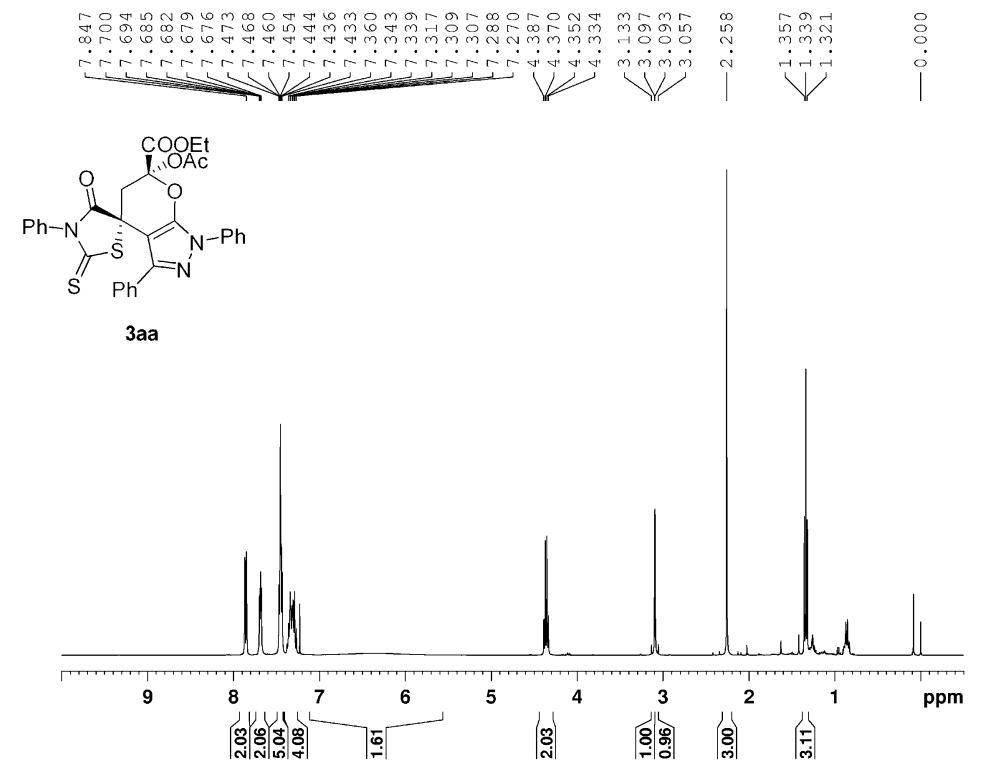

${ }^{13} \mathrm{C}-\mathrm{NMR}$ spectrum of $\mathbf{3 a a}$ in $\mathrm{CDCl}_{3}(100 \mathrm{MHz})$

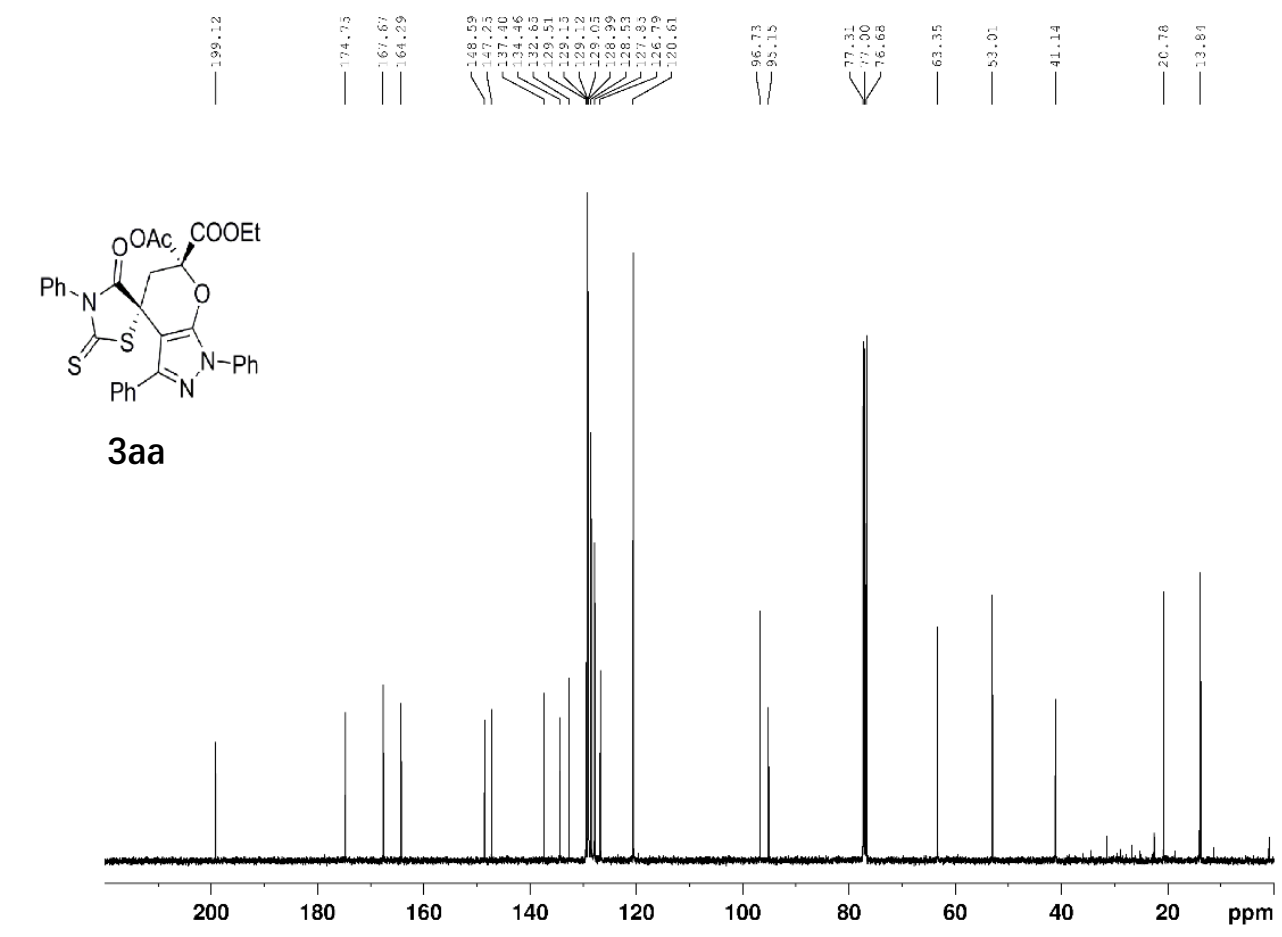


${ }^{1} \mathrm{H}-\mathrm{NMR}$ spectrum of $\mathbf{3 a b}$ in $\mathrm{CDCl}_{3}(600 \mathrm{MHz})$

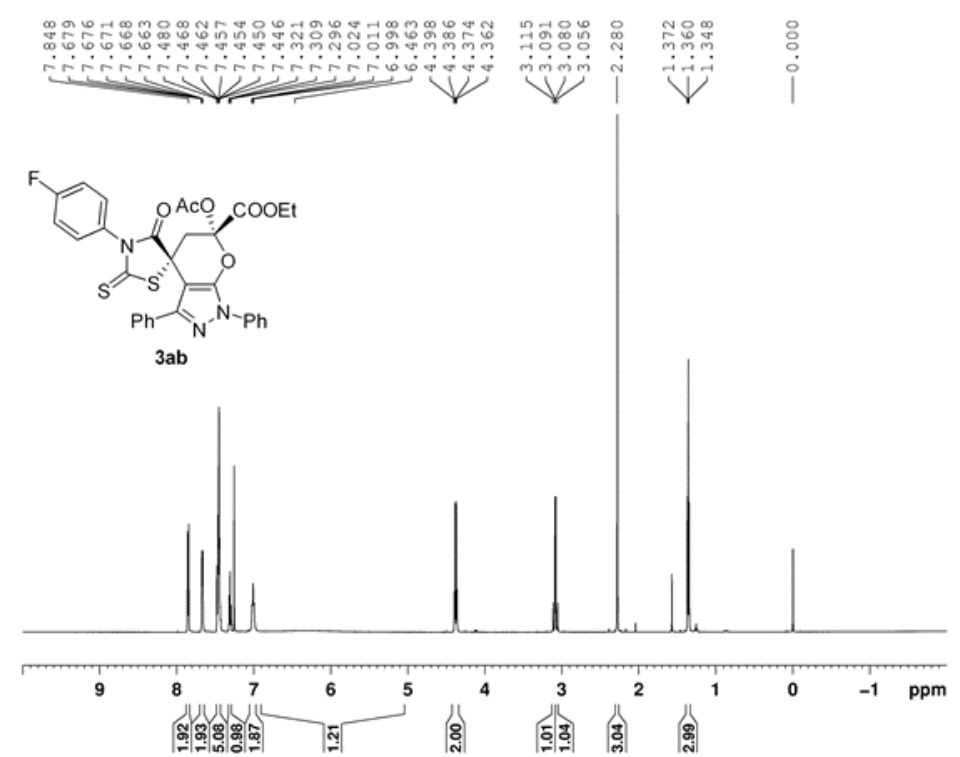

${ }^{13} \mathrm{C}-\mathrm{NMR}$ spectrum in of $\mathbf{3 a b} \mathrm{CDCl}_{3}(150 \mathrm{MHz})$

$\mid$
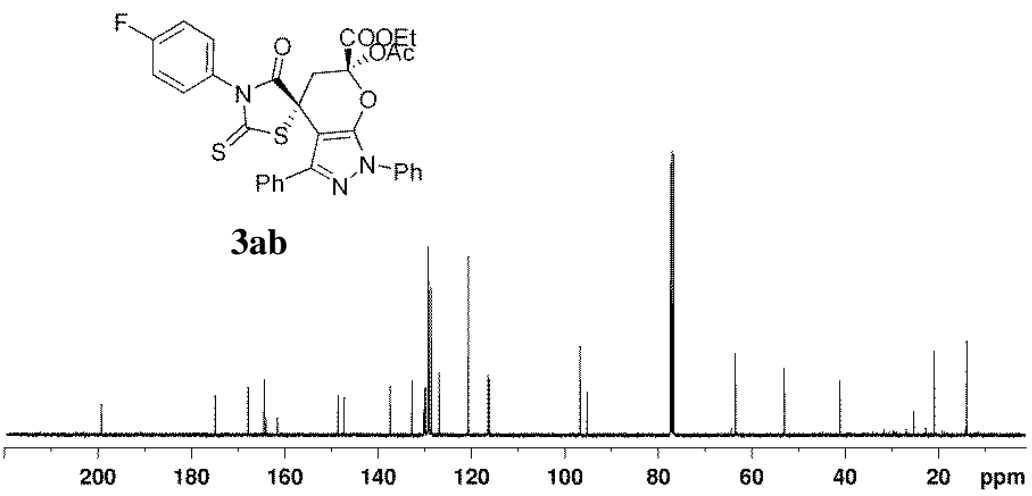
${ }^{1} \mathrm{H}-\mathrm{NMR}$ spectrum of $\mathbf{3 a c}$ in $\mathrm{CDCl}_{3}(600 \mathrm{MHz})$

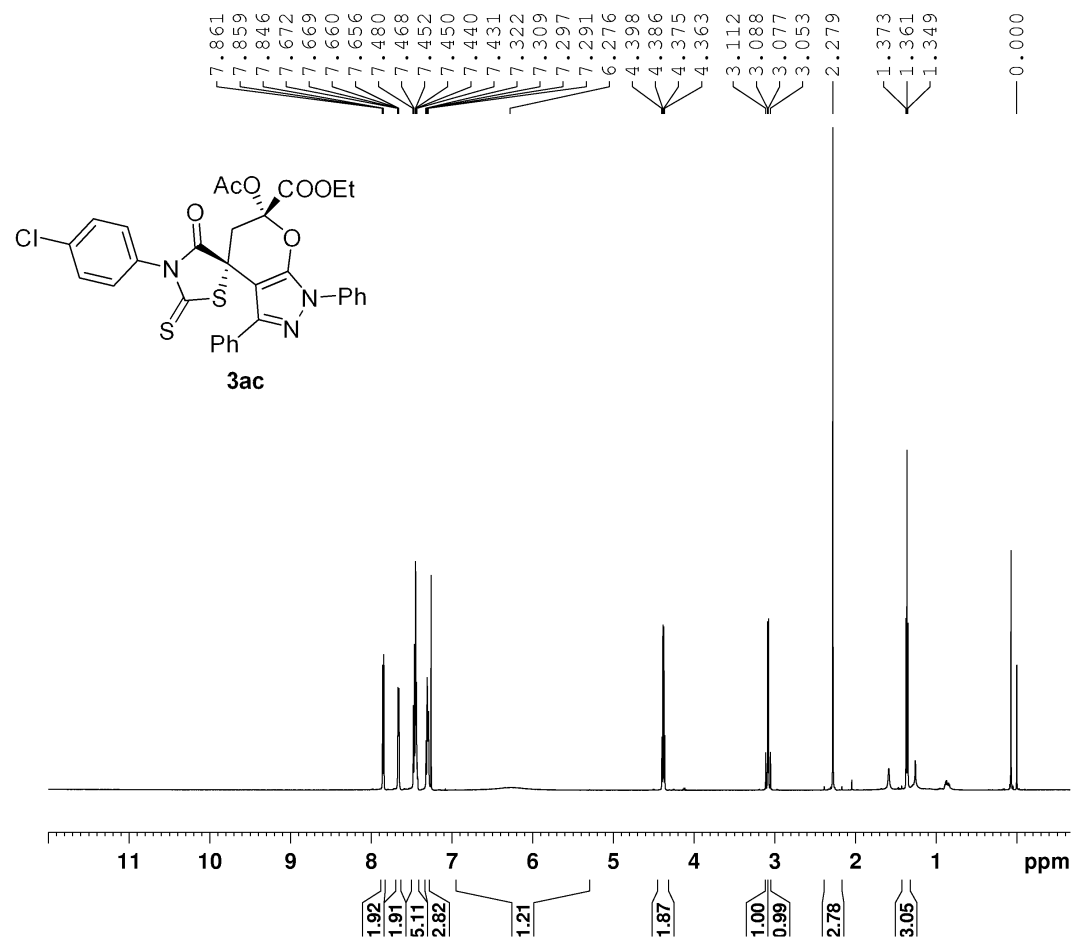

${ }^{13} \mathrm{C}-\mathrm{NMR}$ spectrum of $\mathbf{3 a c}$ in $\mathrm{CDCl}_{3}(150 \mathrm{MHz})$

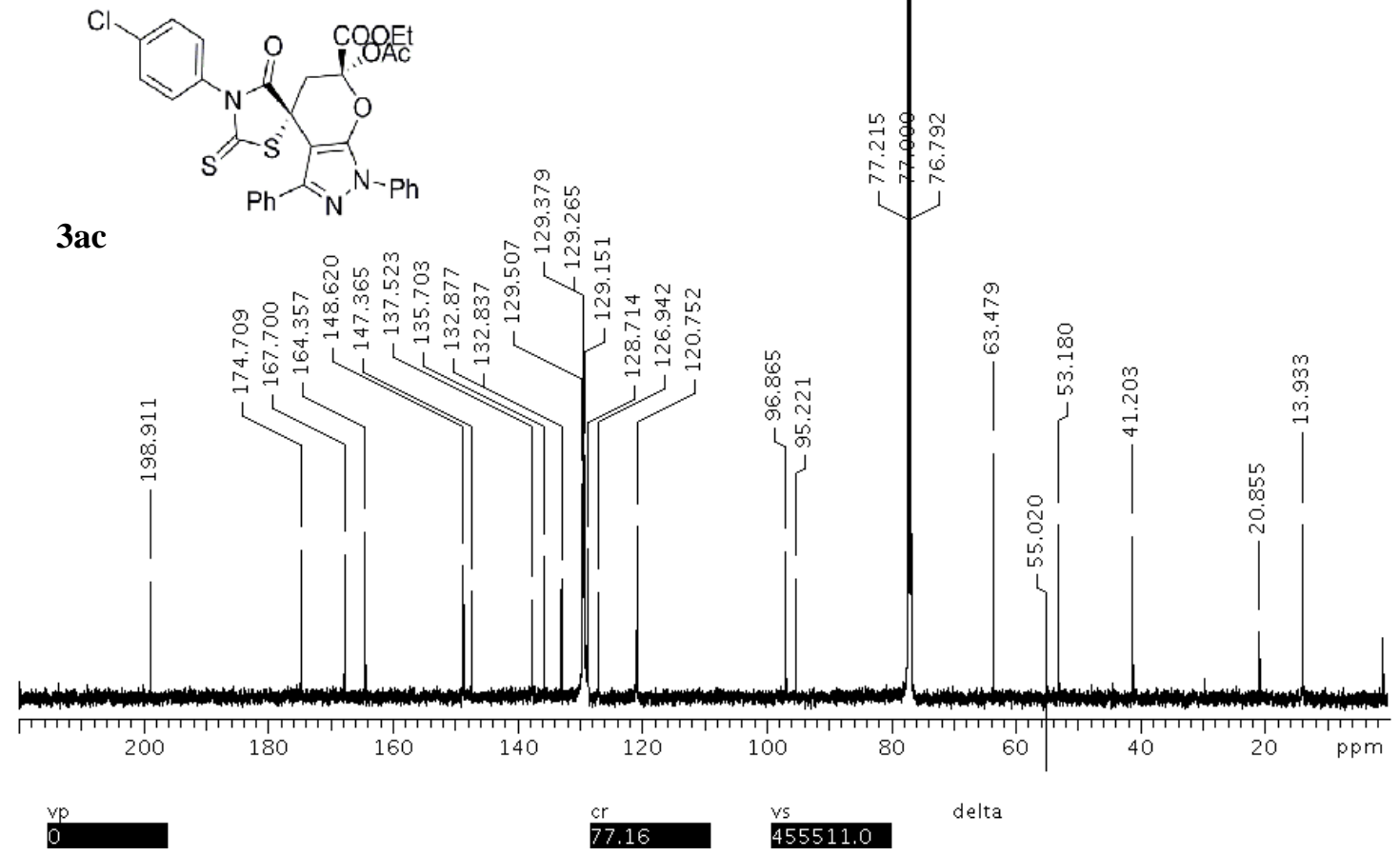


${ }^{1} \mathrm{H}-\mathrm{NMR}$ spectrum of $\mathbf{3 a d}$ in $\mathrm{CDCl}_{3}(600 \mathrm{MHz})$

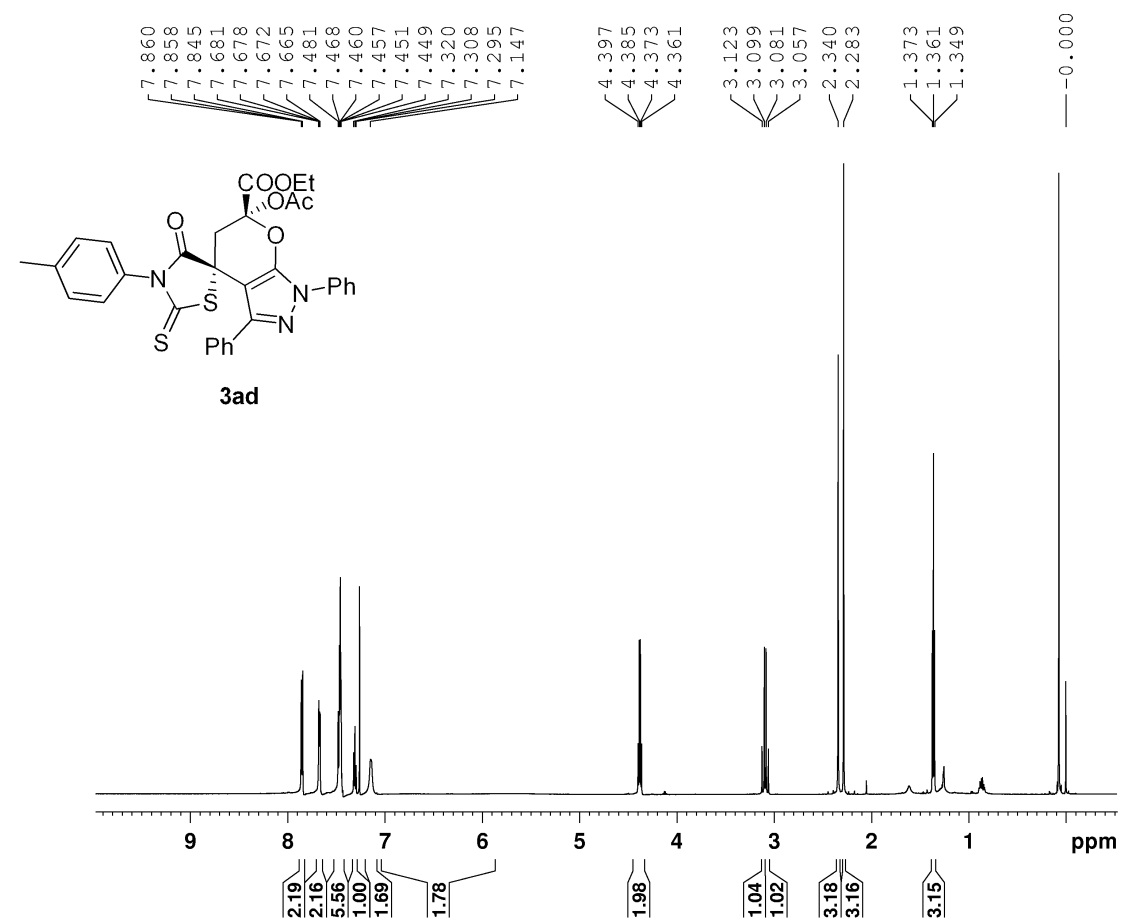

${ }^{13} \mathrm{C}-\mathrm{NMR}$ spectrum of $\mathbf{3 a d}$ in $\mathrm{CDCl}_{3}(150 \mathrm{MHz})$

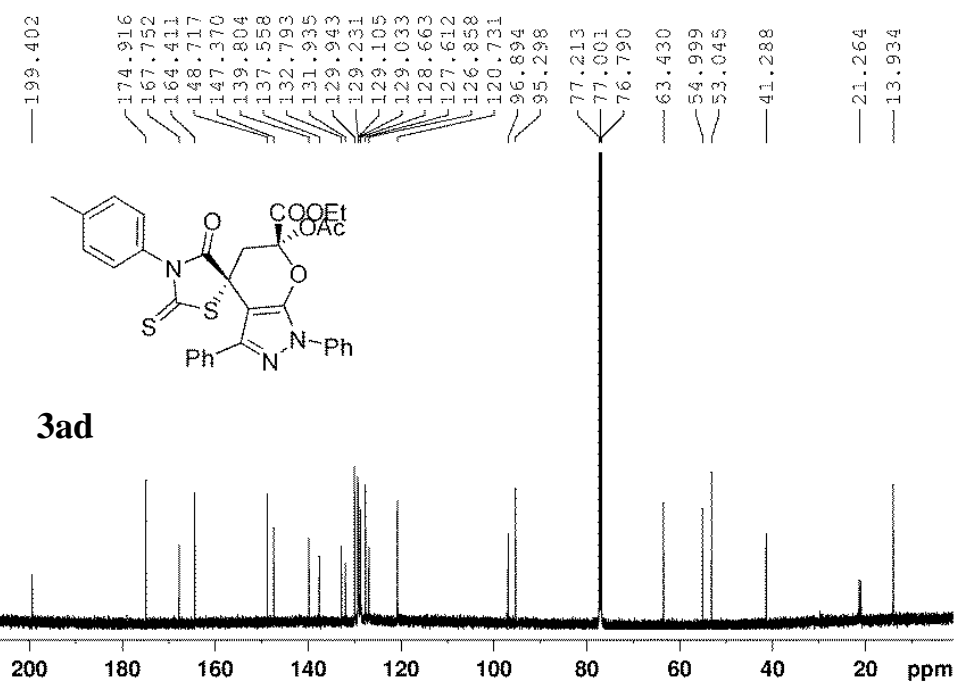


${ }^{1} \mathrm{H}-\mathrm{NMR}$ spectrum of $\mathbf{3 a e}$ in $\mathrm{CDCl}_{3}(400 \mathrm{MHz})$

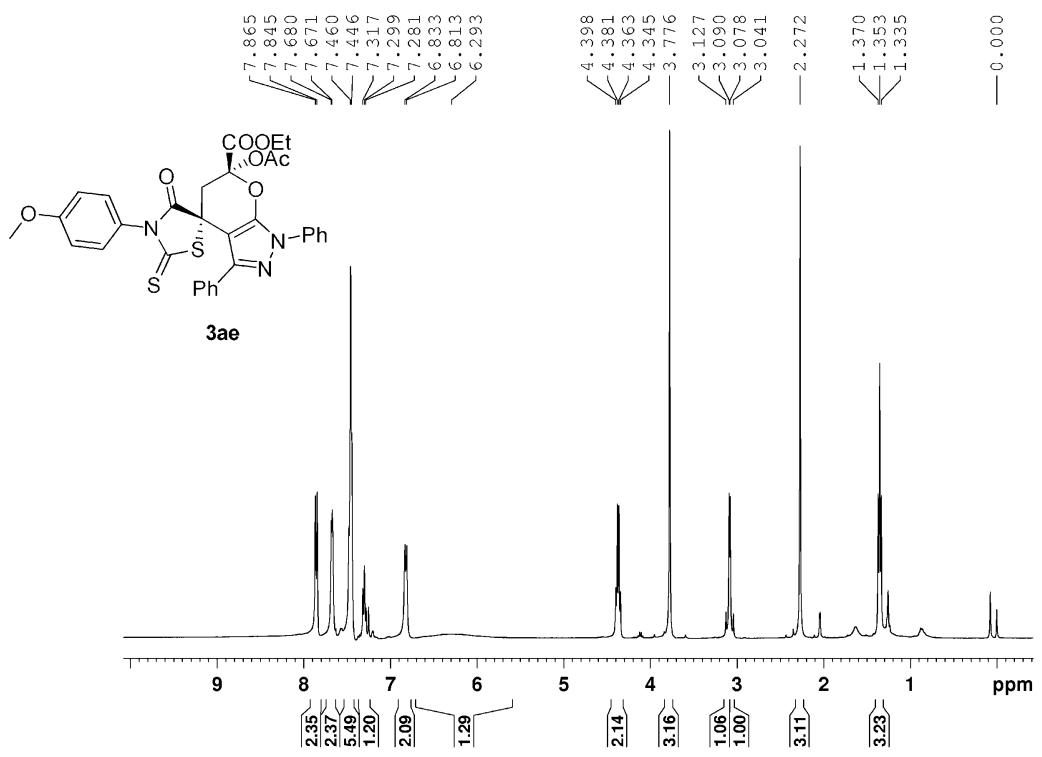

${ }^{13} \mathrm{C}-\mathrm{NMR}$ spectrum of $\mathbf{3 a e}$ in $\mathrm{CDCl}_{3}(100 \mathrm{MHz})$

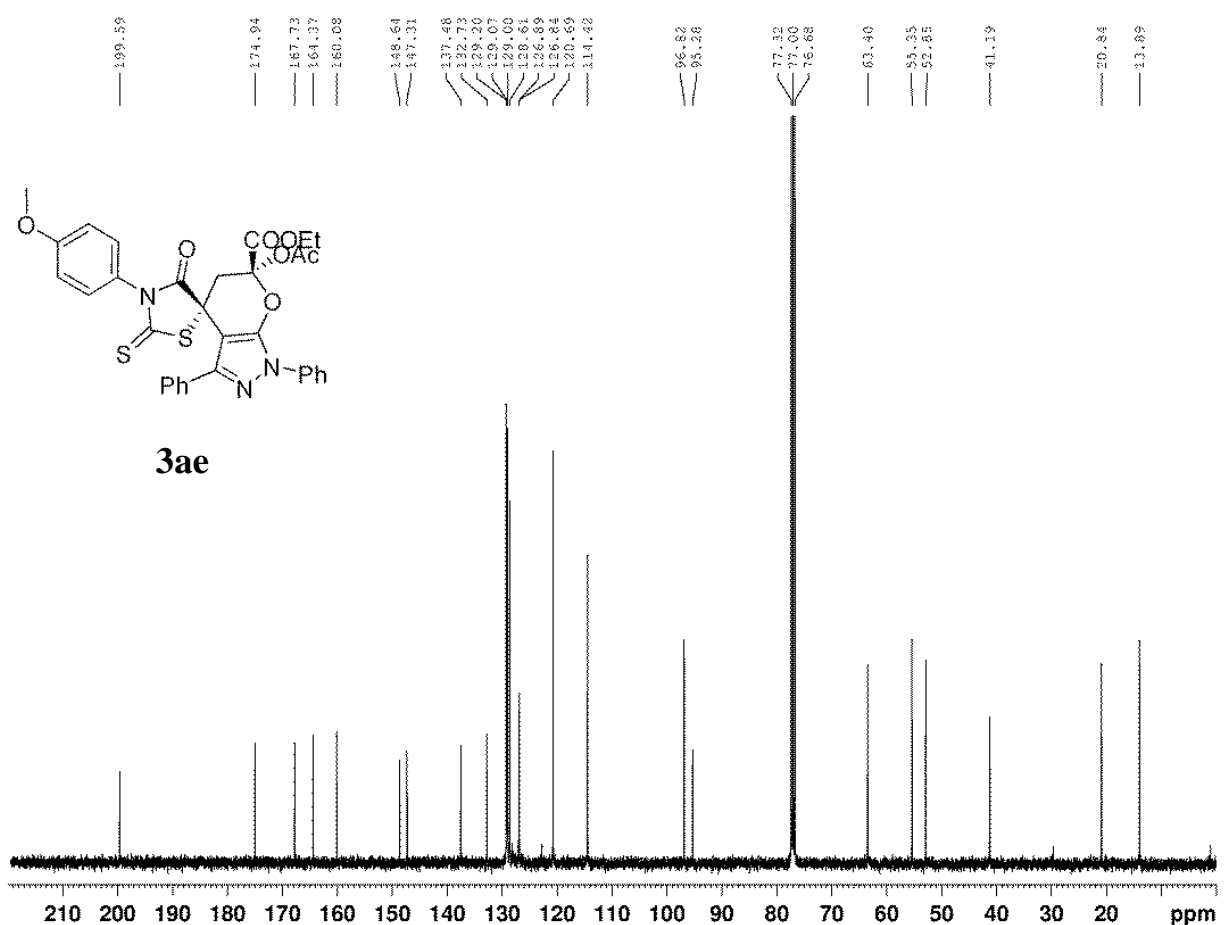


${ }^{1} \mathrm{H}-\mathrm{NMR}$ spectrum of $\mathbf{3 b a}$ in of $\mathrm{CDCl}_{3}(600 \mathrm{MHz})$

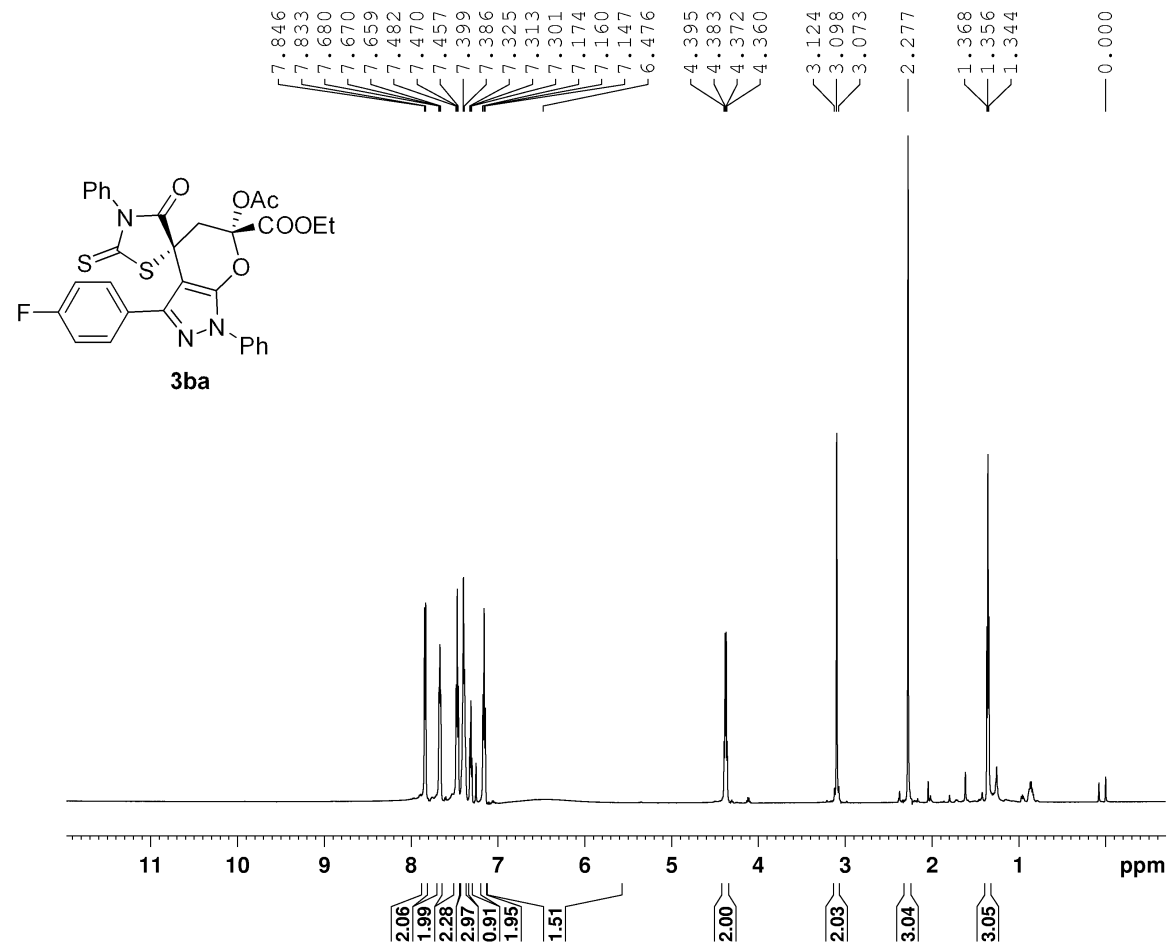

${ }^{13} \mathrm{C}-\mathrm{NMR}$ spectrum of $\mathbf{3 b a}$ in $\mathrm{CDCl}_{3}(150 \mathrm{MHz})$

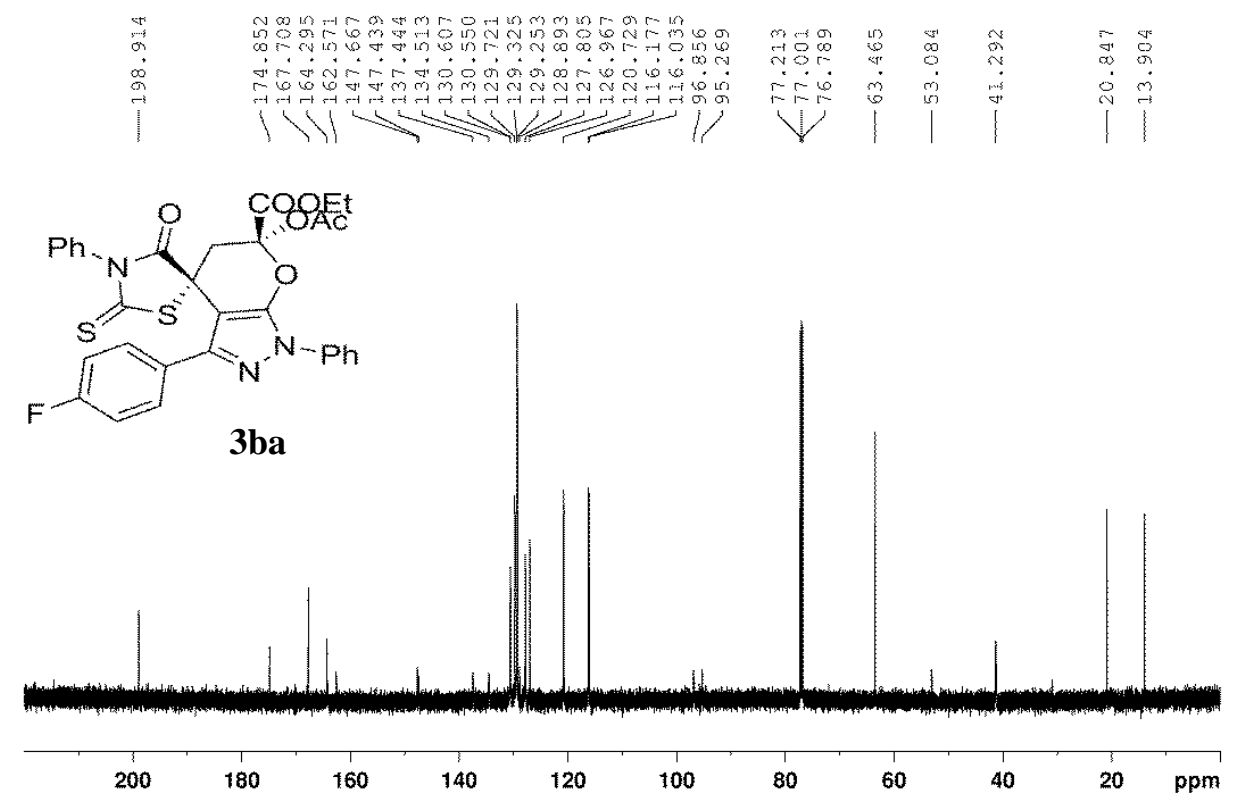


${ }^{1} \mathrm{H}-\mathrm{NMR}$ spectrum of $\mathbf{3 c a}$ in $\mathrm{CDCl}_{3}(600 \mathrm{MHz})$

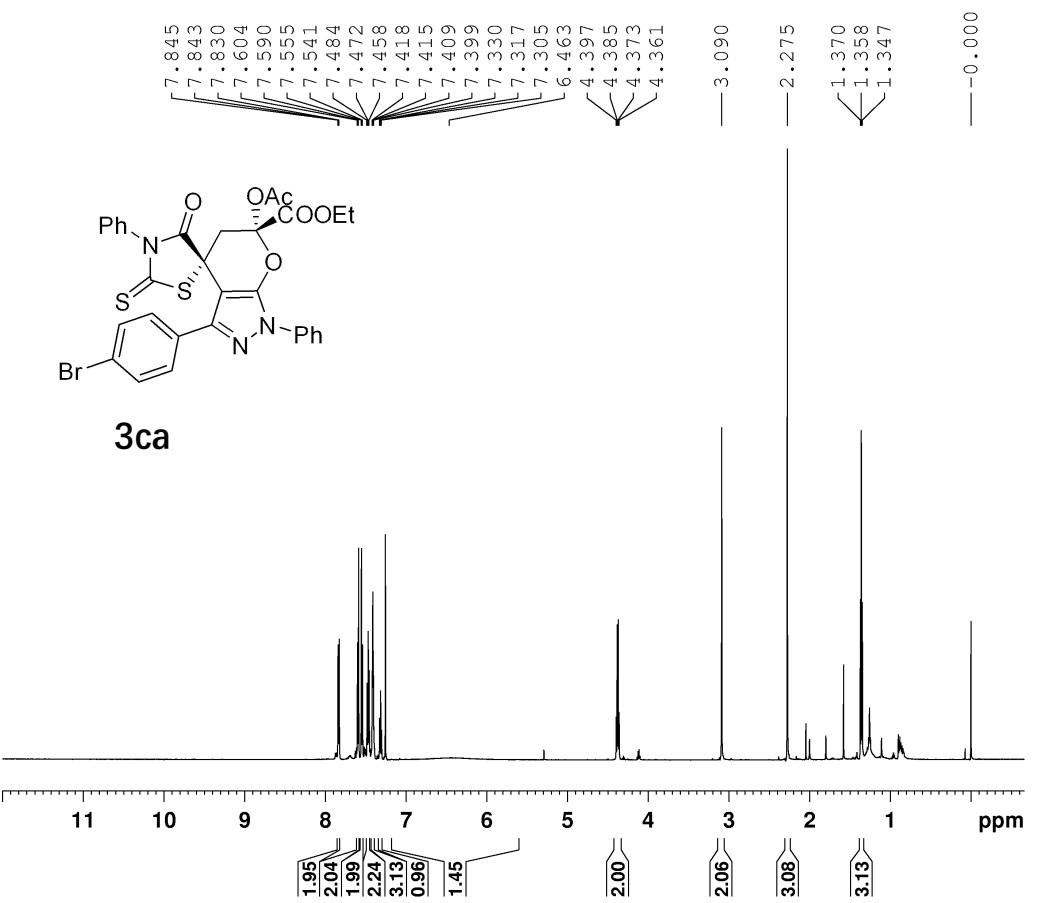

${ }^{13} \mathrm{C}-\mathrm{NMR}$ spectrum of $\mathbf{3 c a}$ in $\mathrm{CDCl}_{3}(150 \mathrm{MHz})$

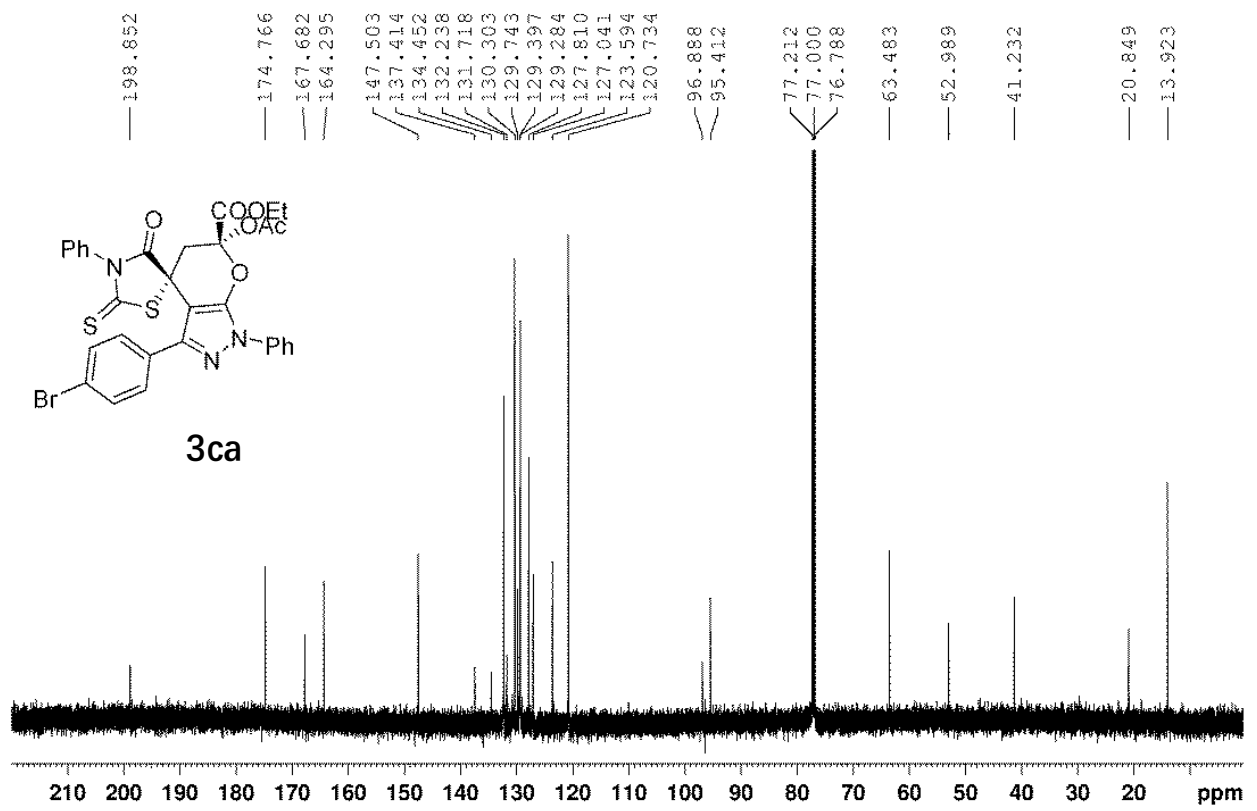


${ }^{1} \mathrm{H}-\mathrm{NMR}$ spectrum of $\mathbf{3 d a}$ in $\mathrm{CDCl}_{3}(600 \mathrm{MHz})$

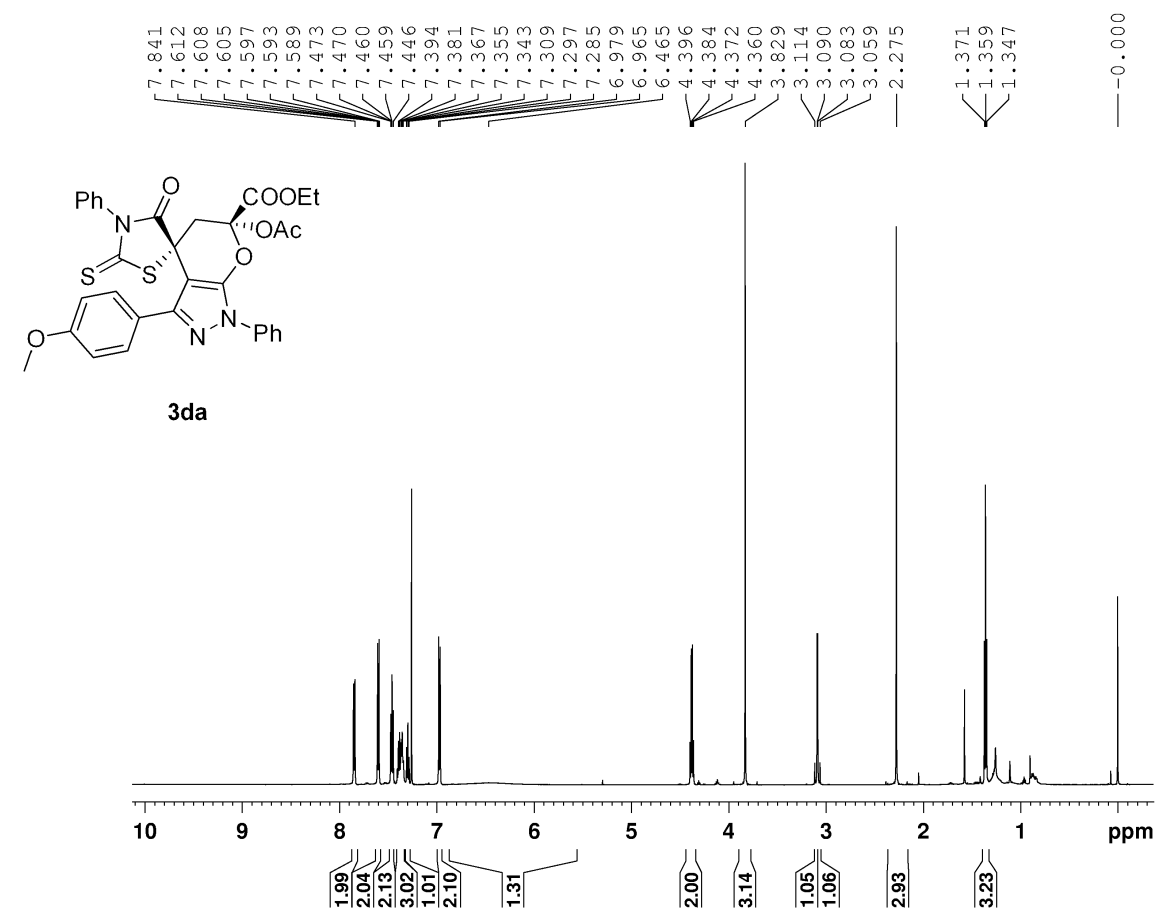

${ }^{13} \mathrm{C}-\mathrm{NMR}$ spectrum of $\mathbf{3 d a}$ in $\mathrm{CDCl}_{3}(150 \mathrm{MHz})$

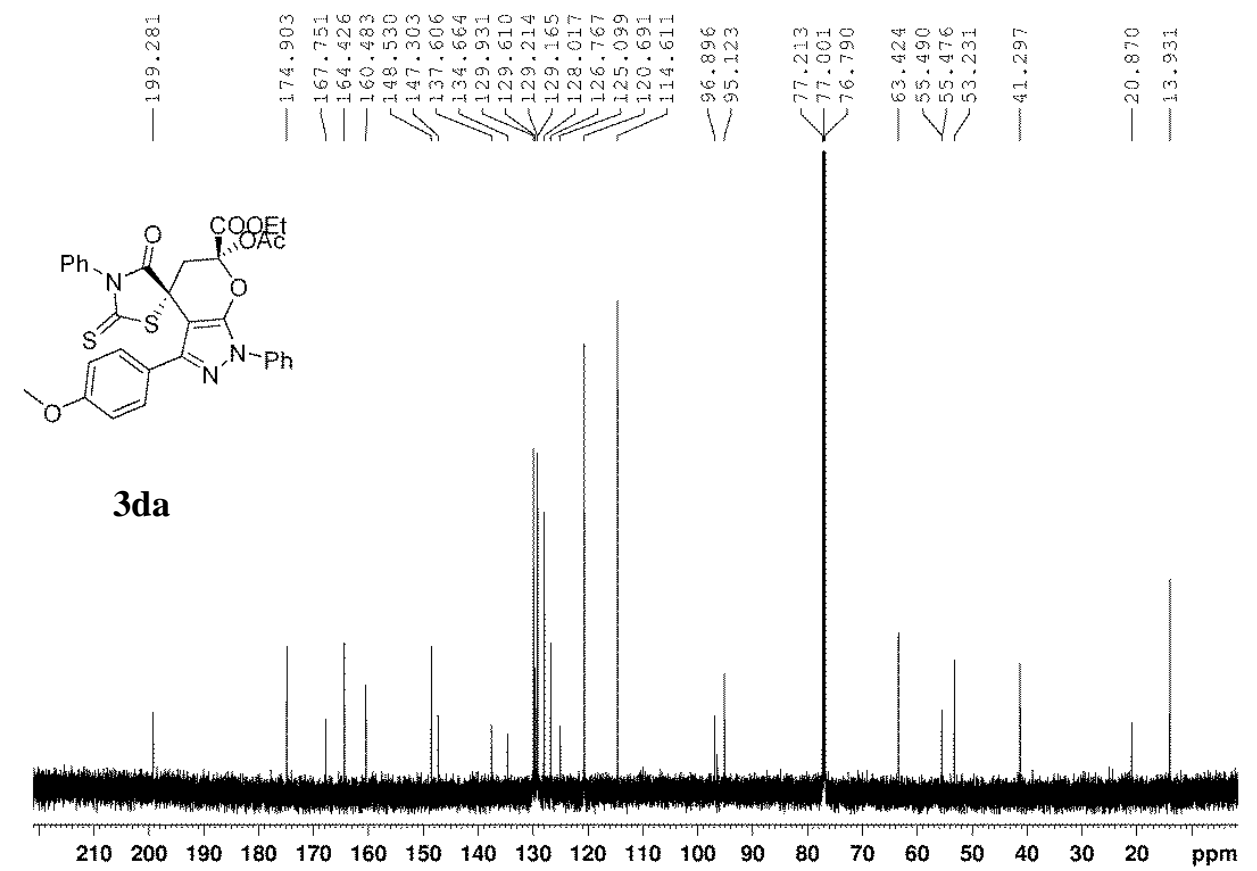


${ }^{1} \mathrm{H}-\mathrm{NMR}$ spectrum of 3ea in $\mathrm{CDCl}_{3}(400 \mathrm{MHz})$

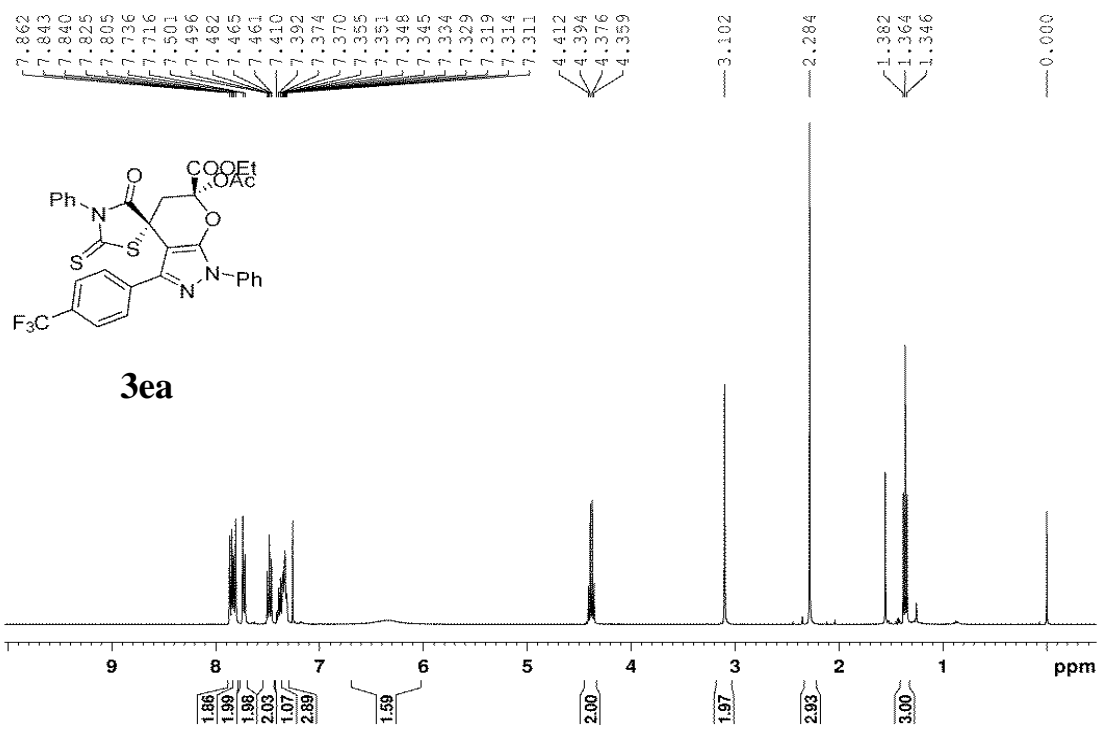

${ }^{13} \mathrm{C}$-NMR spectrum of $\mathbf{3 e a}$ in $\mathrm{CDCl}_{3}(100 \mathrm{MHz})$

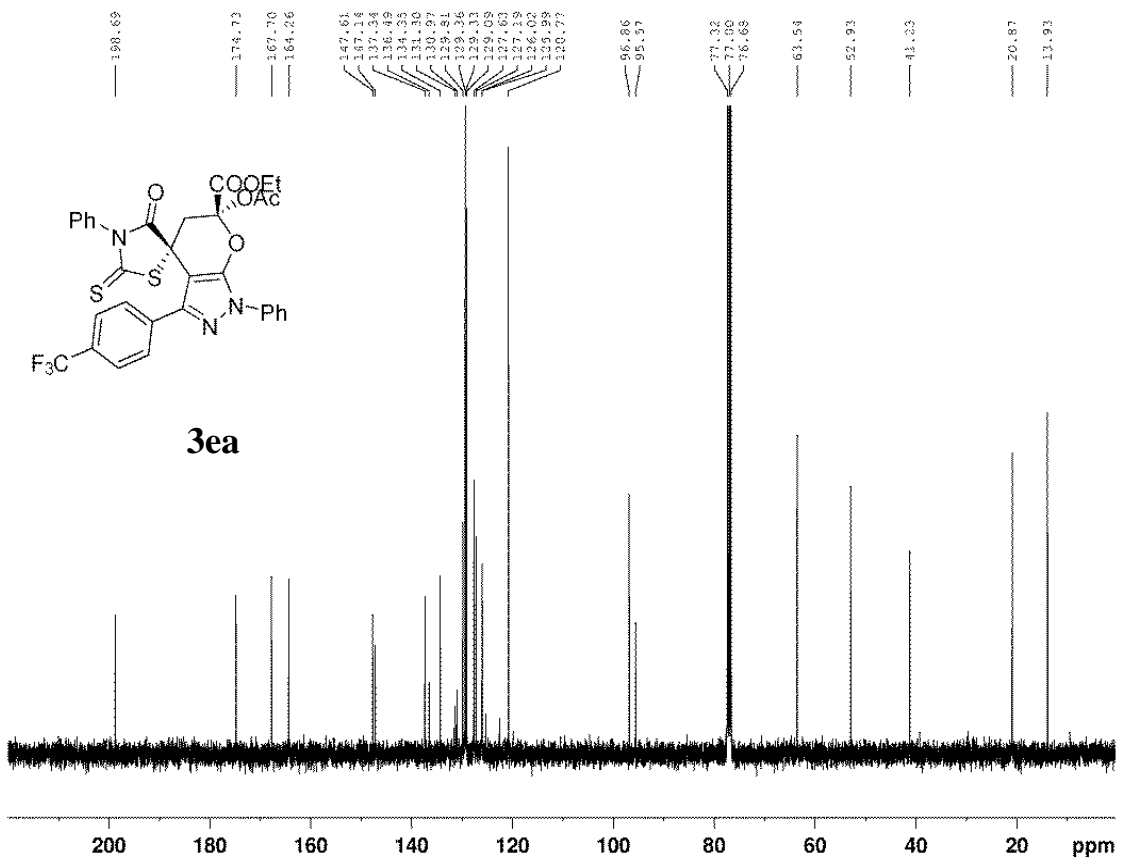


${ }^{1} \mathrm{H}-\mathrm{NMR}$ spectrum of $\mathbf{3 f a}$ in $\mathrm{CDCl}_{3}(600 \mathrm{MHz})$

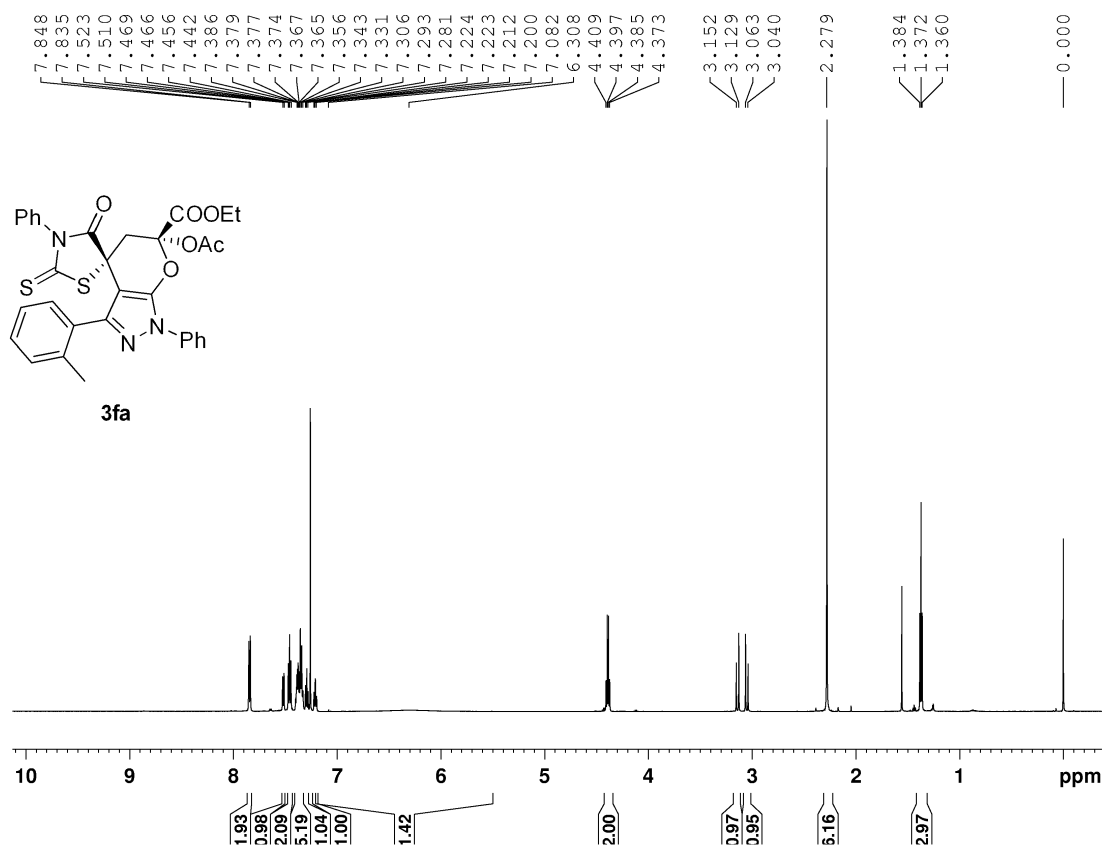

${ }^{13} \mathrm{C}-\mathrm{NMR}$ spectrum of $\mathbf{3 f a}$ in $\mathrm{CDCl}_{3}(150 \mathrm{MHz})$

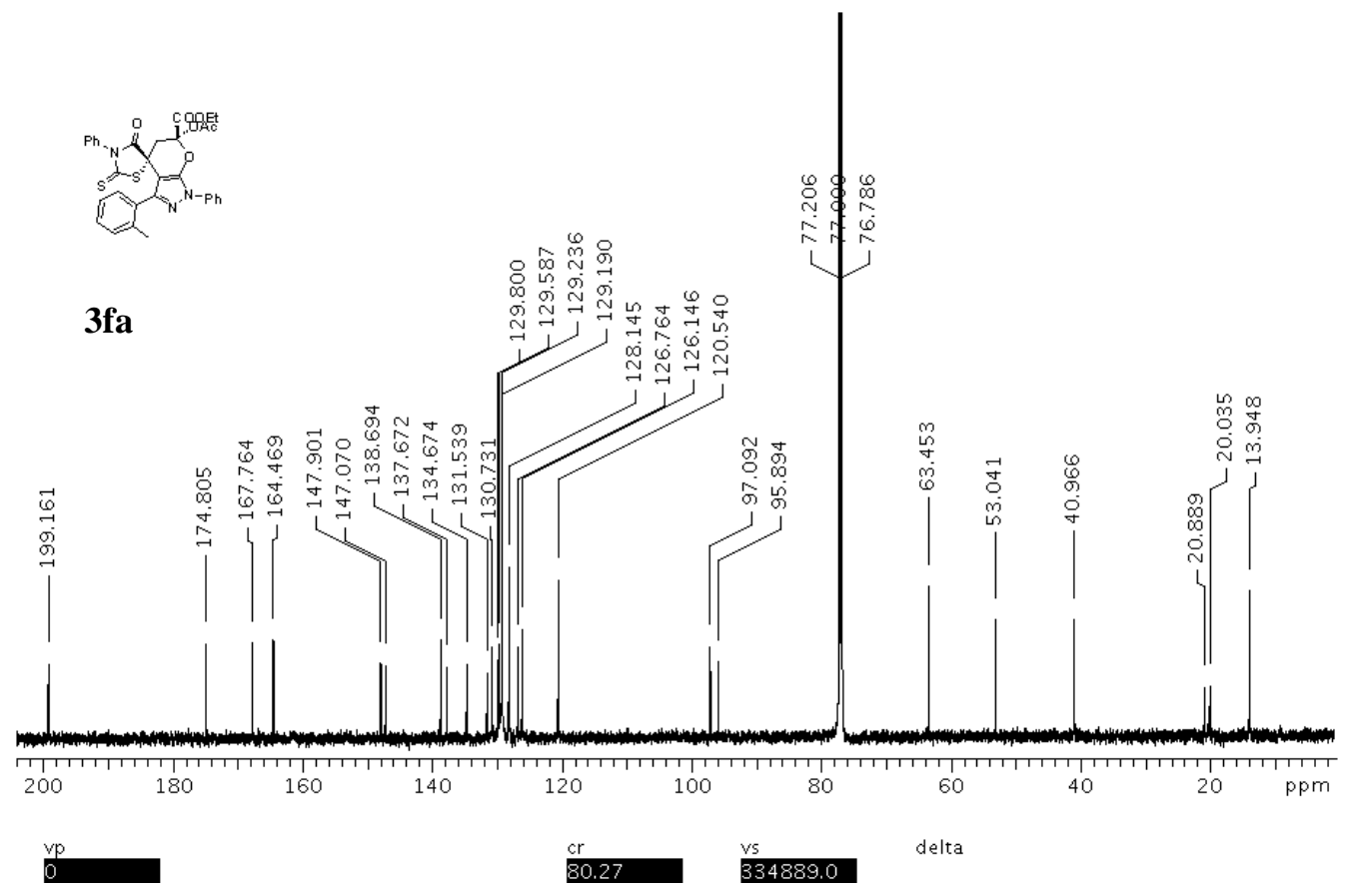


${ }^{1} \mathrm{H}-\mathrm{NMR}$ spectrum of $\mathbf{3 g a}$ in $\mathrm{CDCl}_{3}(400 \mathrm{MHz})$

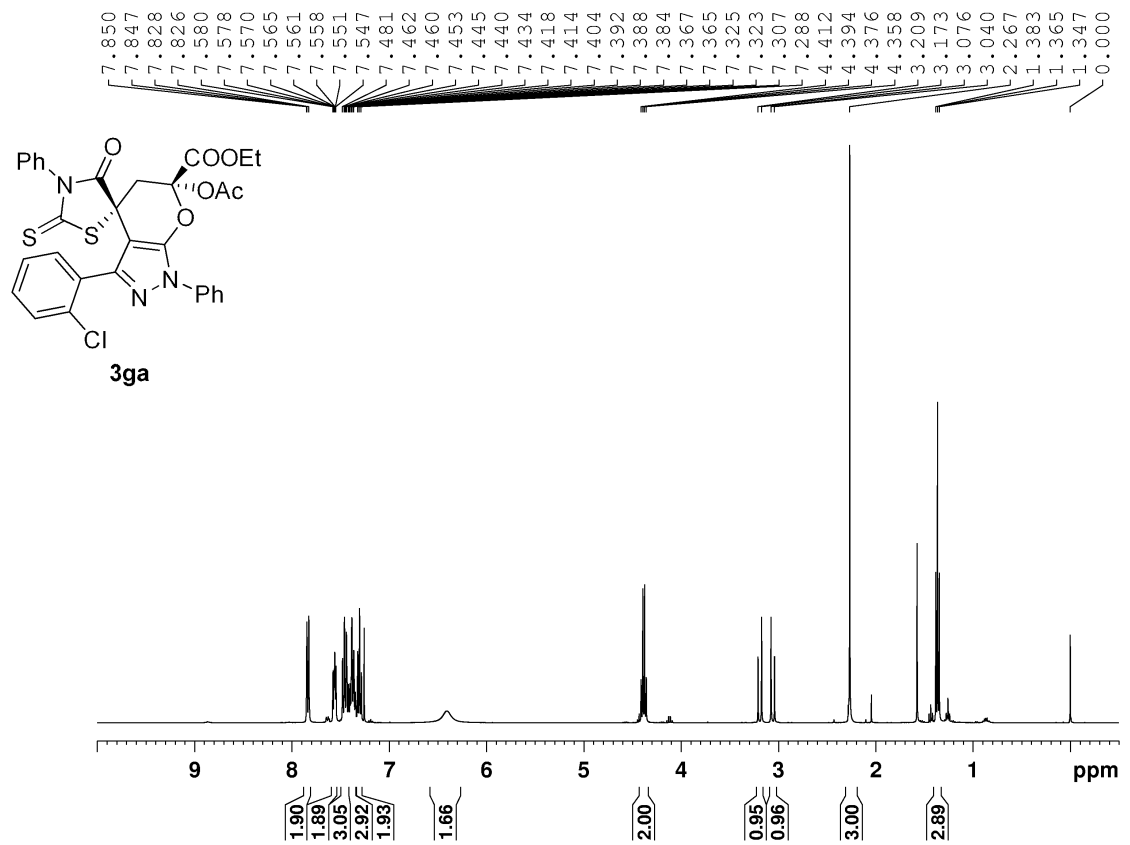

${ }^{13} \mathrm{C}-\mathrm{NMR}$ spectrum of $\mathbf{3 g a}$ in $\mathrm{CDCl}_{3}(100 \mathrm{MHz})$

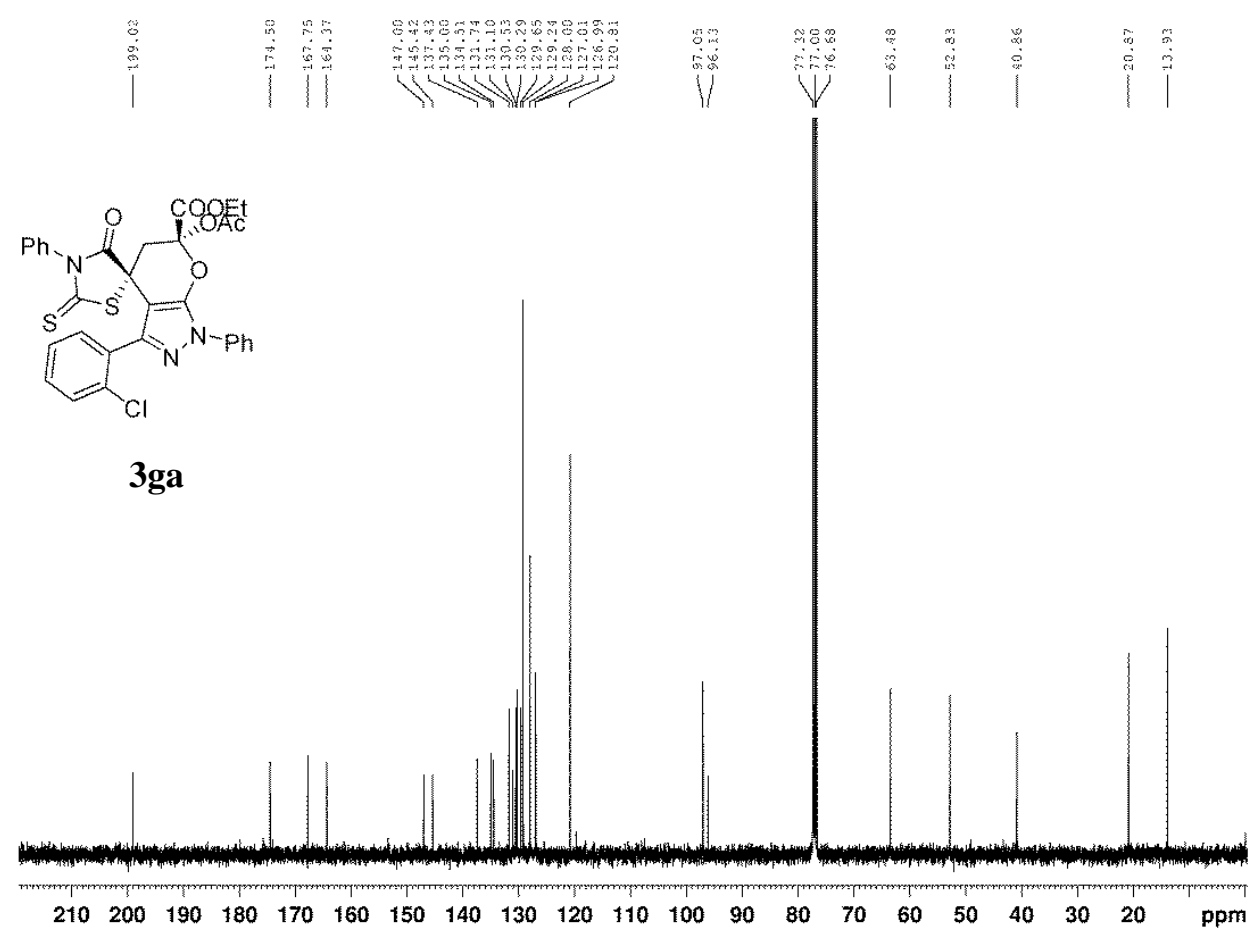


${ }^{1} \mathrm{H}-\mathrm{NMR}$ spectrum of $\mathbf{3 h a}$ in $\mathrm{CDCl}_{3}(600 \mathrm{MHz})$

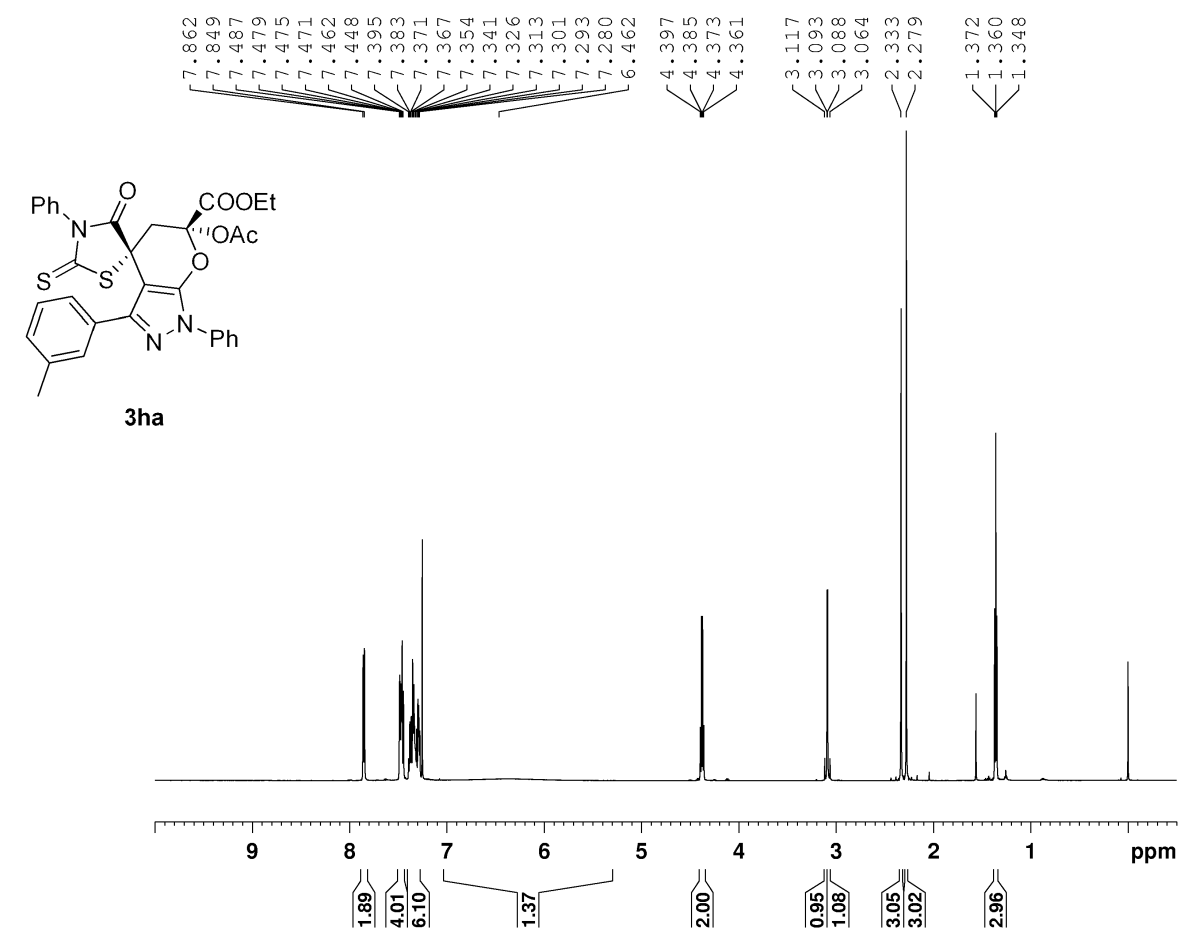

${ }^{13} \mathrm{C}-\mathrm{NMR}$ spectrum of $\mathbf{3 h a}$ in $\mathrm{CDCl}_{3}(150 \mathrm{MHz})$

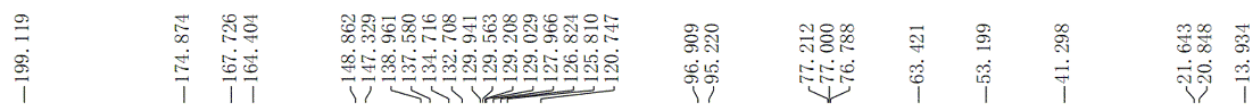
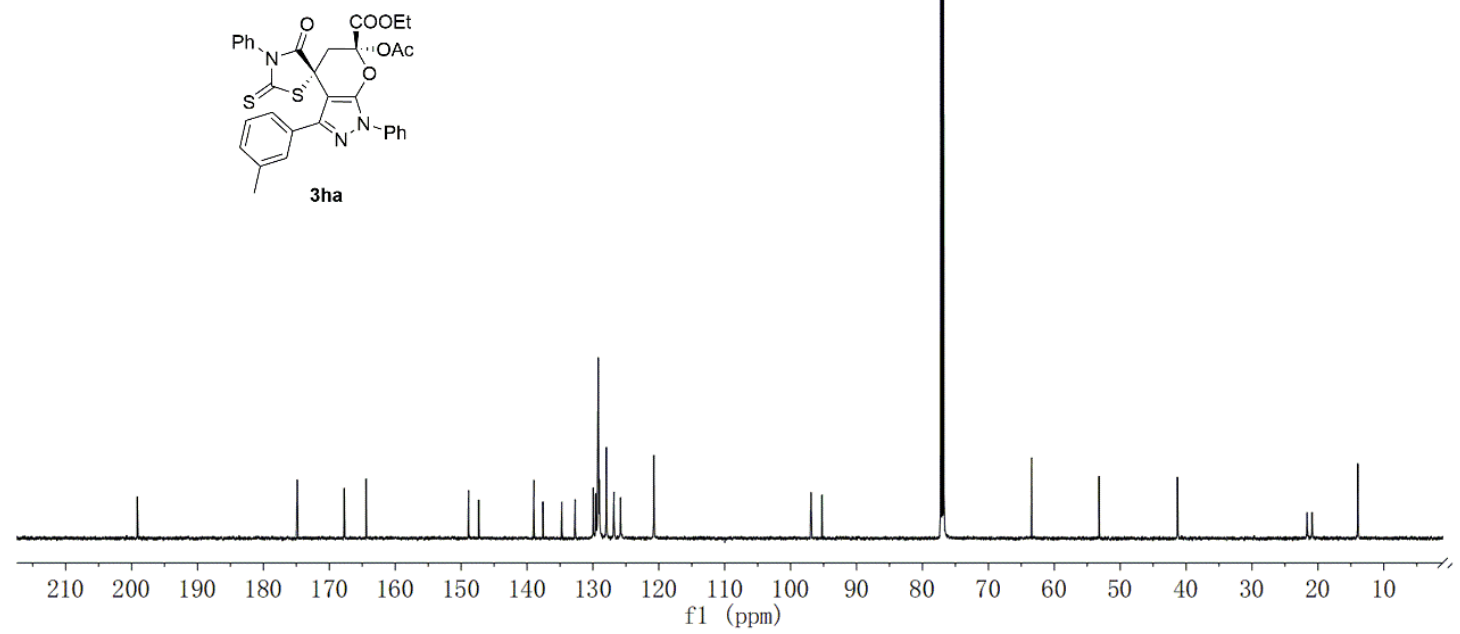
${ }^{1} \mathrm{H}-\mathrm{NMR}$ spectrum of 3ia in $\mathrm{CDCl}_{3}(400 \mathrm{MHz})$

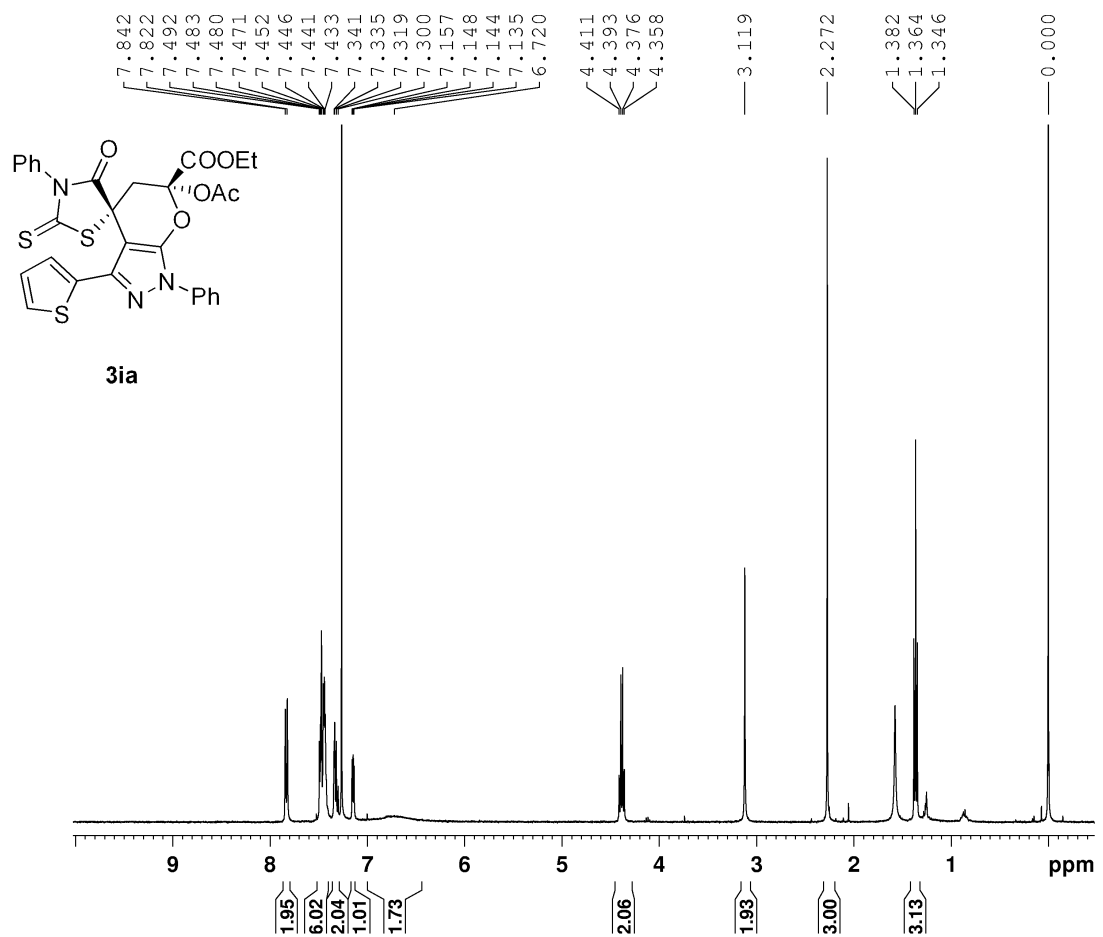

${ }^{13} \mathrm{C}$-NMR spectrum of $\mathbf{3 i a}$ in $\mathrm{CDCl}_{3}(150 \mathrm{MHz})$<smiles>CCOC(=O)[C@]1(C)C[C@@]2(SC(=S)N(c3ccccc3)C2=O)c2c(-c3cccs3)nn(-c3ccccc3)c2O1</smiles>
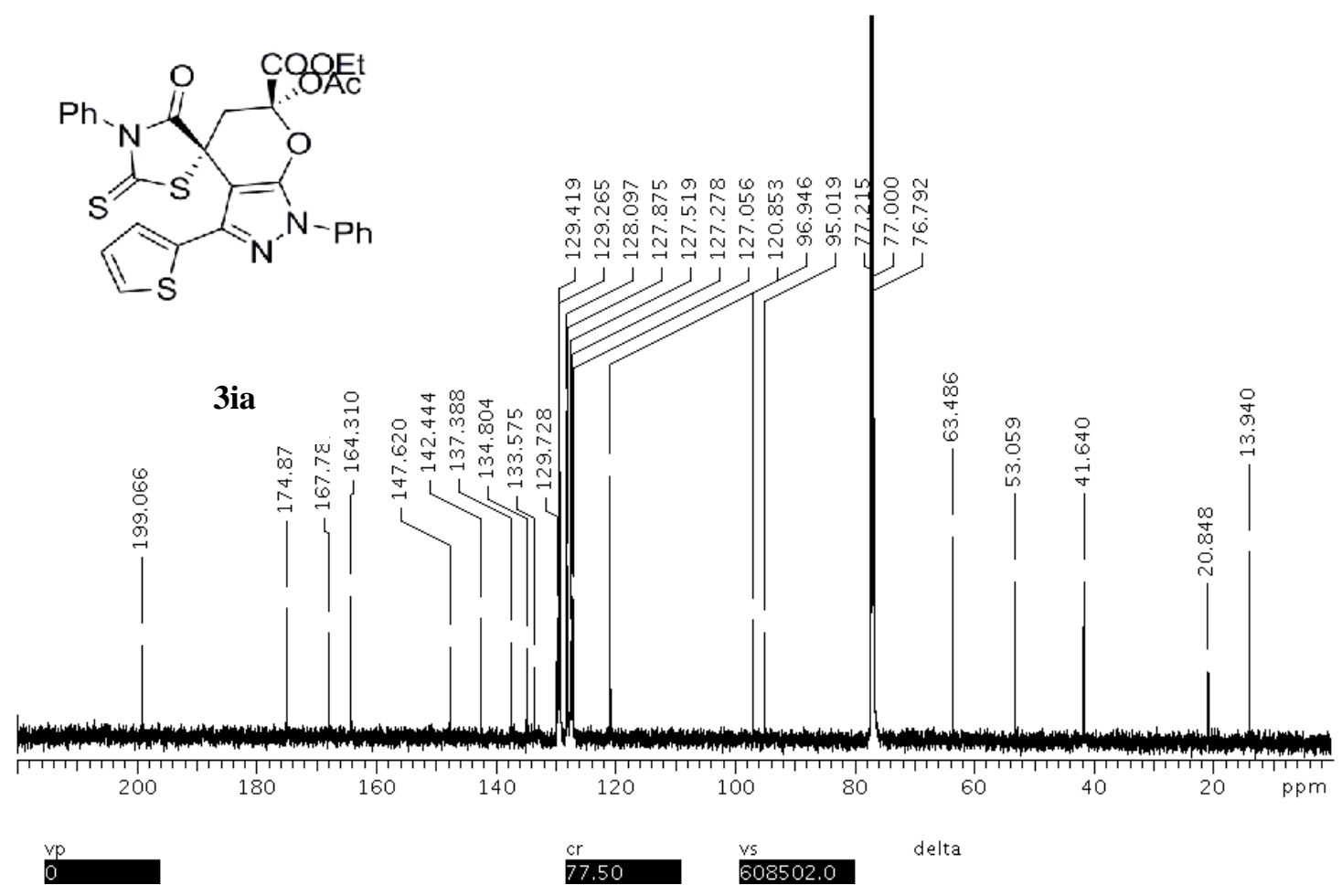
${ }^{1} \mathrm{H}-\mathrm{NMR}$ spectrum of $\mathbf{3} \mathbf{j a}$ in $\mathrm{CDCl}_{3}(400 \mathrm{MHz})$
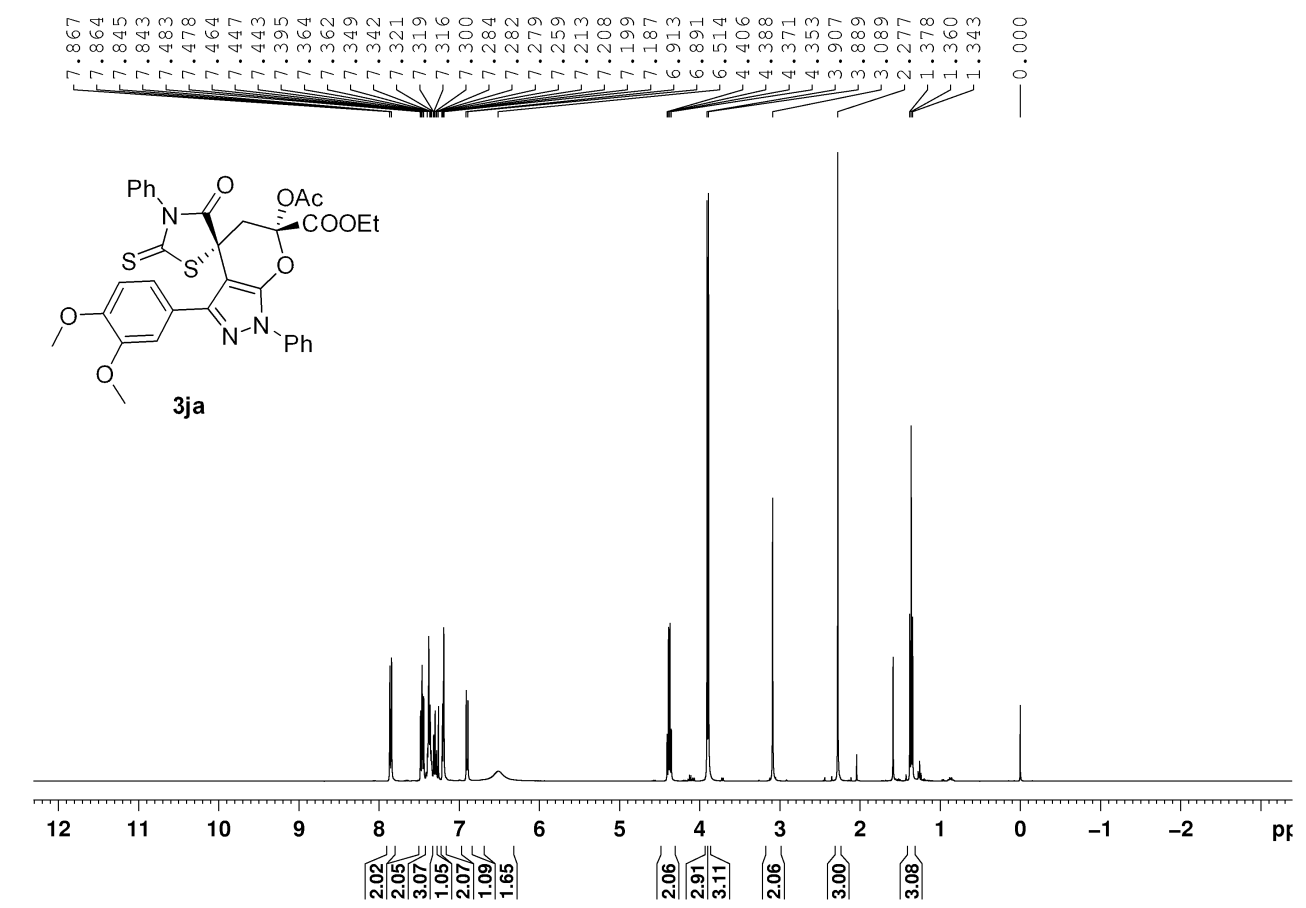

${ }^{13} \mathrm{C}-\mathrm{NMR}$ spectrum of $\mathbf{3} \mathbf{j a}$ in $\mathrm{CDCl}_{3}(100 \mathrm{MHz})$

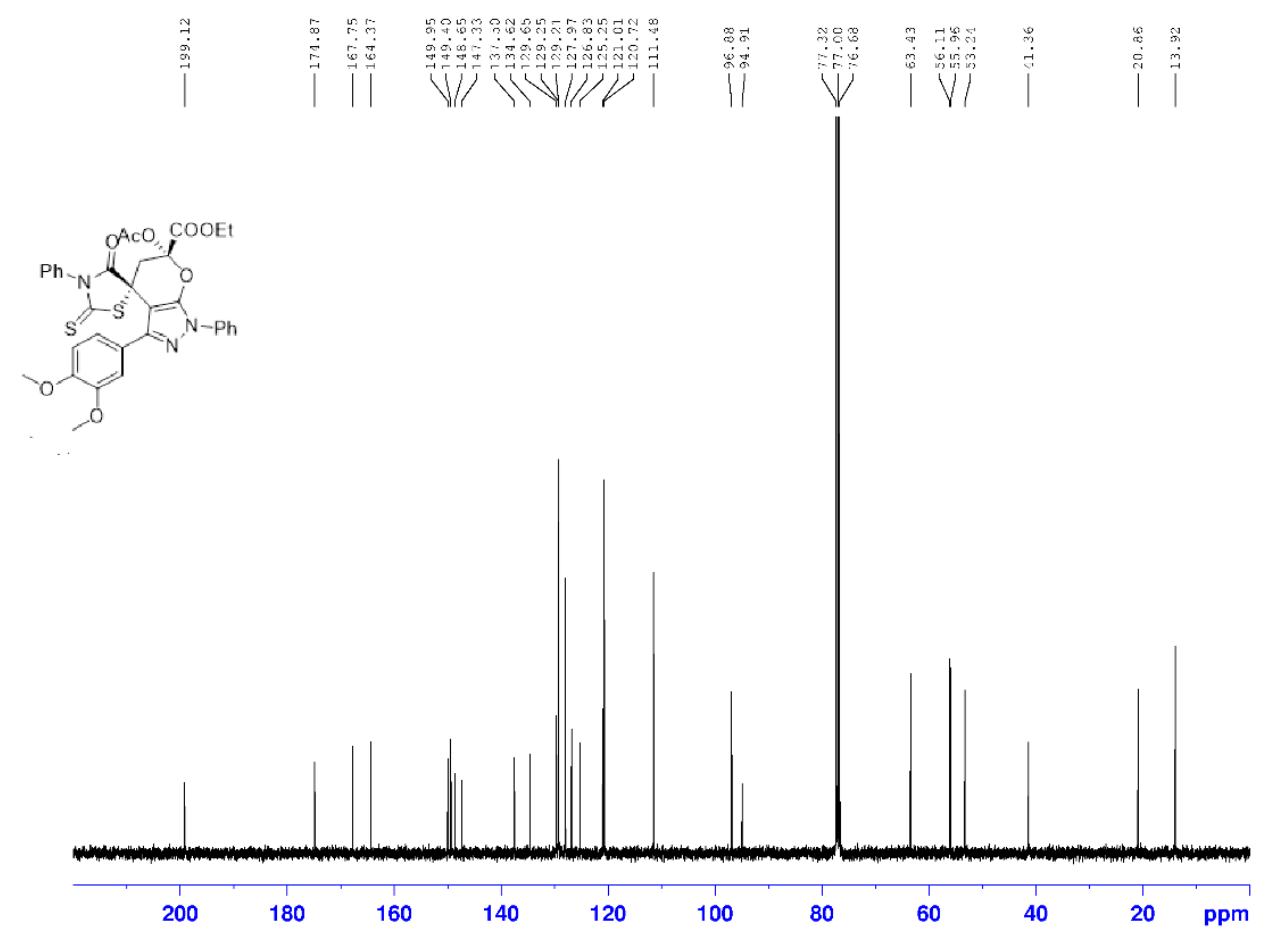


${ }^{1} \mathrm{H}-\mathrm{NMR}$ spectrum of $\mathbf{3 k a}$ in $\mathrm{CDCl}_{3}(400 \mathrm{MHz})$

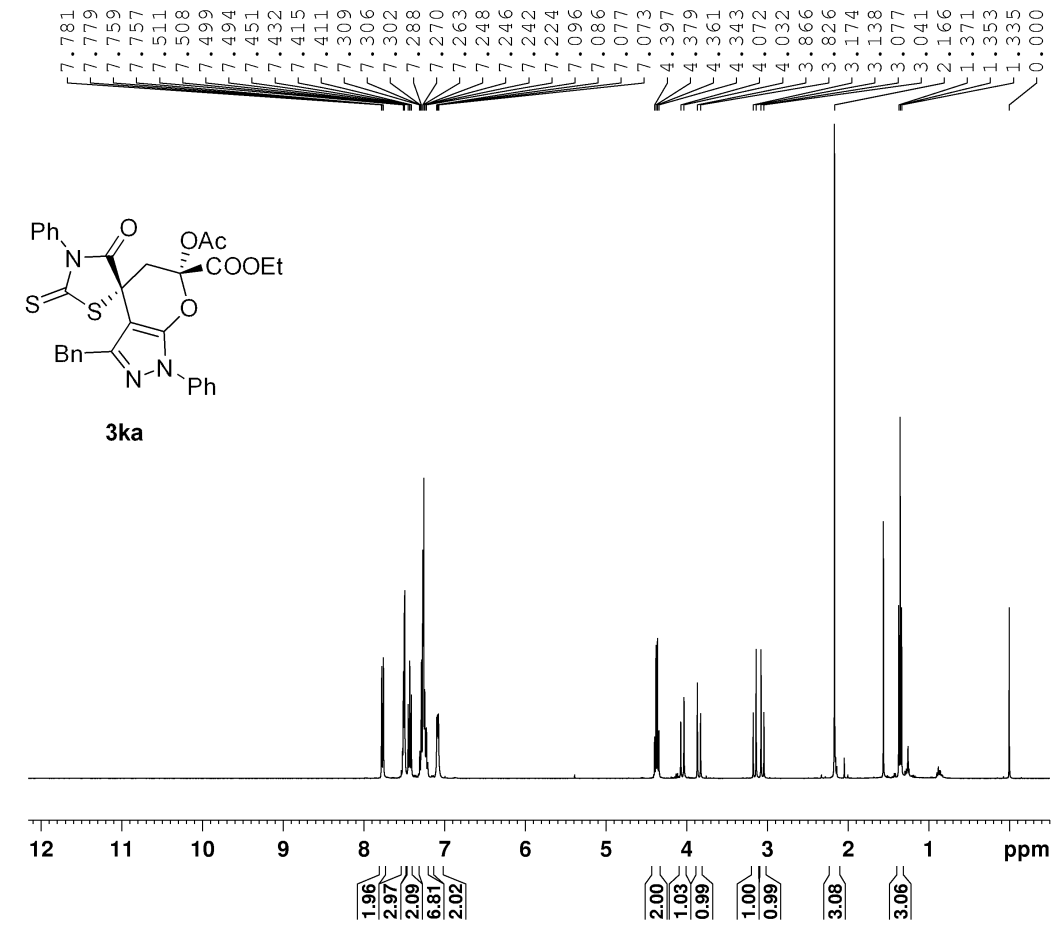

${ }^{13} \mathrm{C}-\mathrm{NMR}$ spectrum of $\mathbf{3 k a}$ in $\mathrm{CDCl}_{3}(100 \mathrm{MHz})$

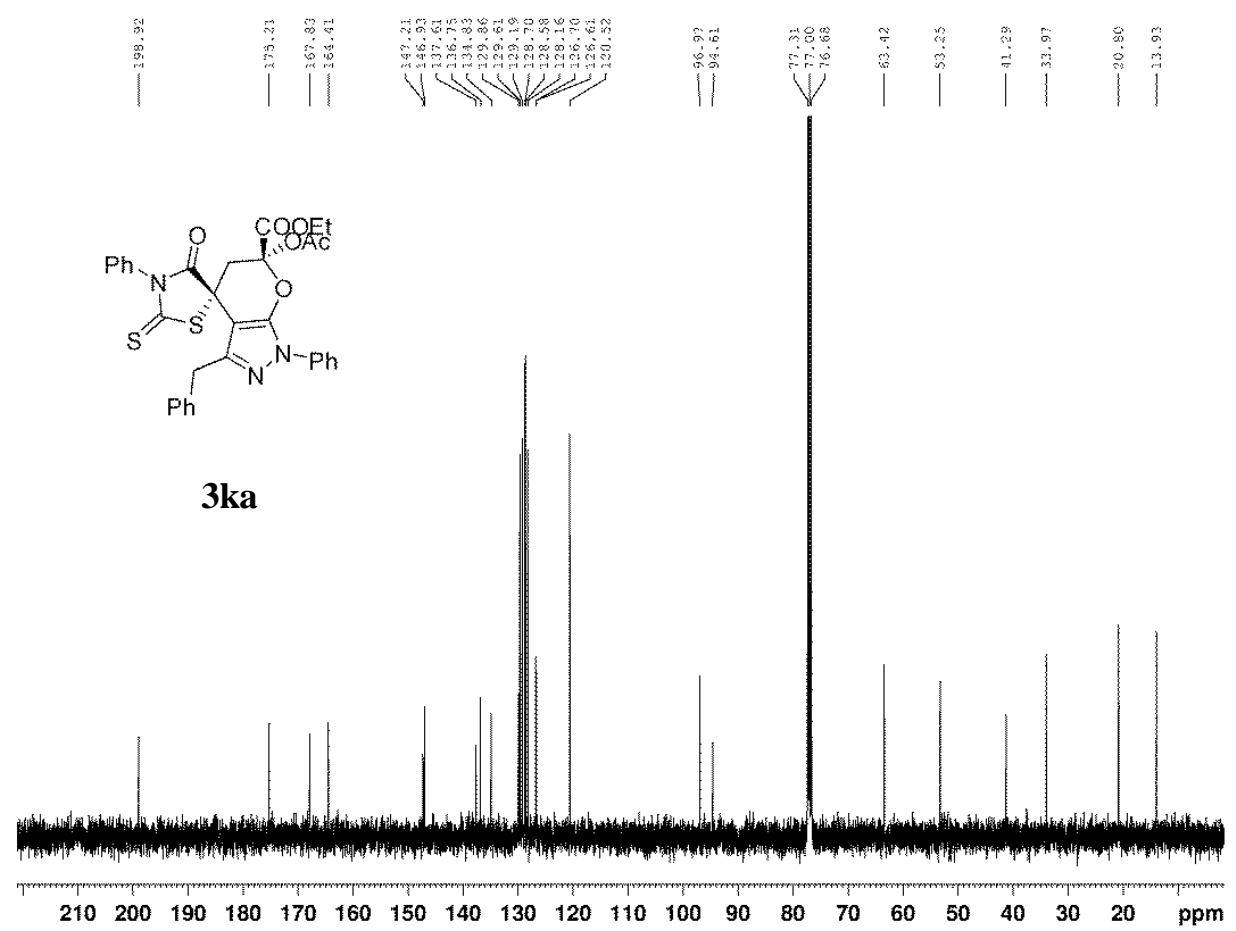


${ }^{1} \mathrm{H}-\mathrm{NMR}$ spectrum of 3la in $\mathrm{CDCl}_{3}(400 \mathrm{MHz})$

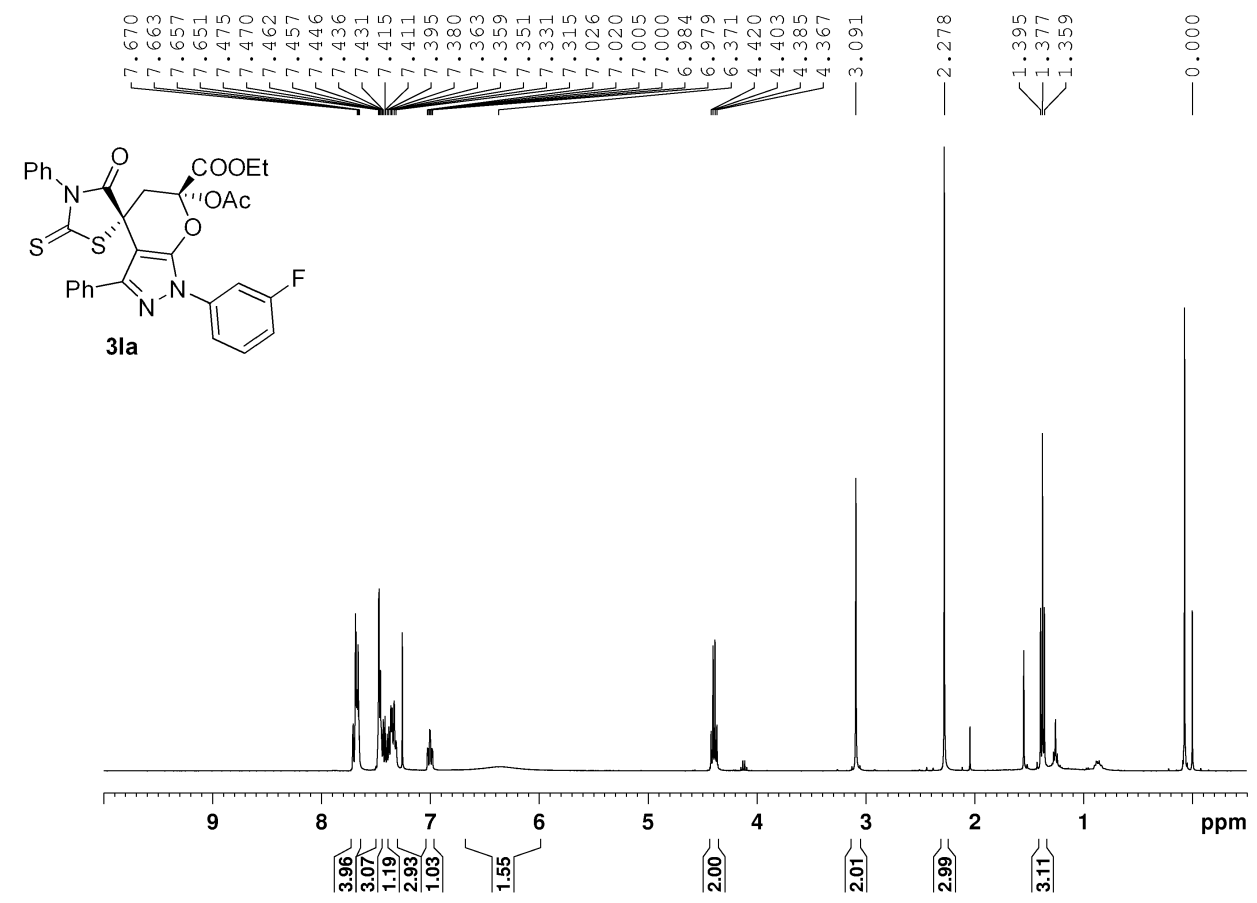

${ }^{13} \mathrm{C}-\mathrm{NMR}$ spectrum of $\mathbf{3 l a}$ in $\mathrm{CDCl}_{3}(100 \mathrm{MHz})$

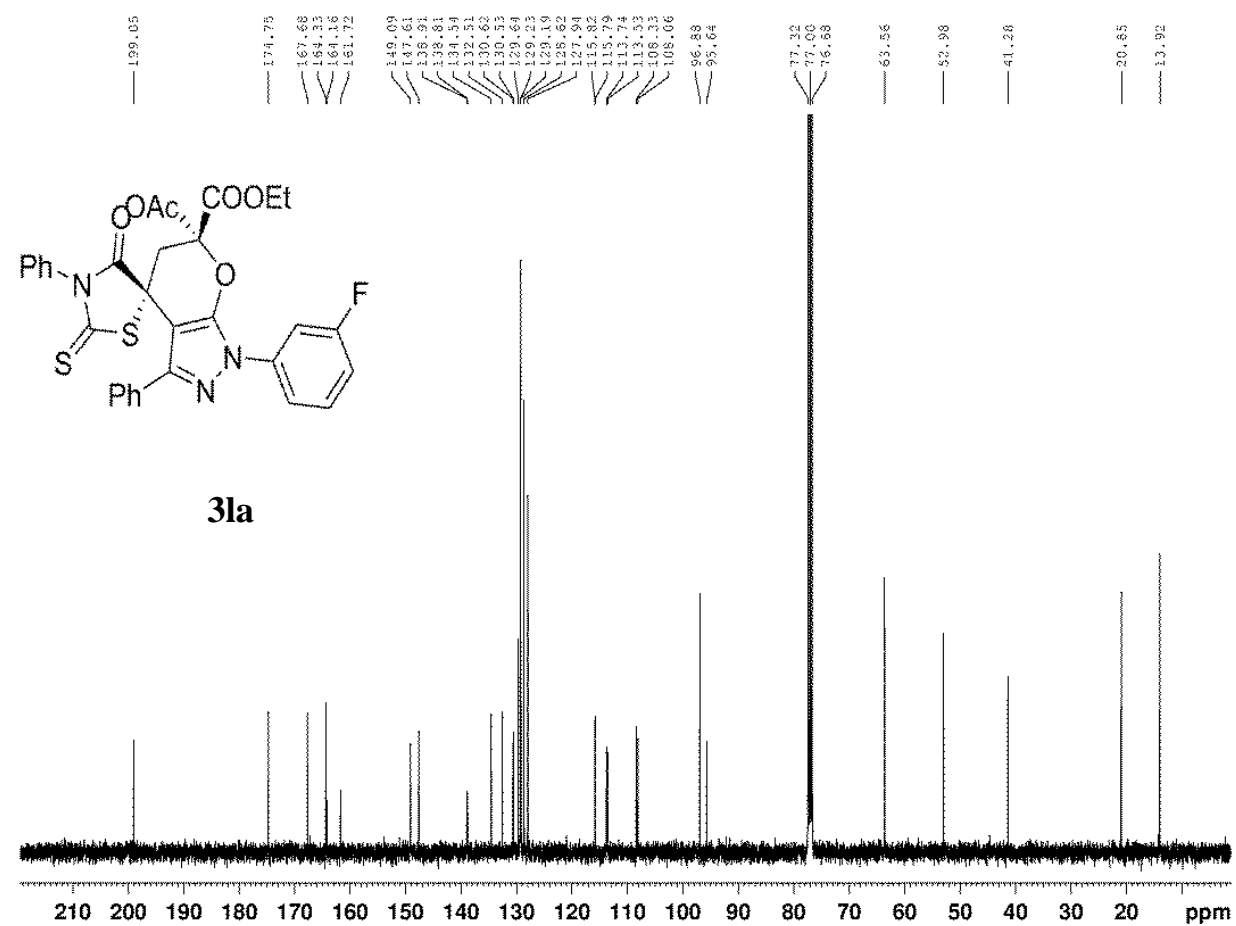


${ }^{1} \mathrm{H}-\mathrm{NMR}$ spectrum of $\mathbf{3} \mathbf{m a}$ in $\mathrm{CDCl}_{3}(400 \mathrm{MHz})$

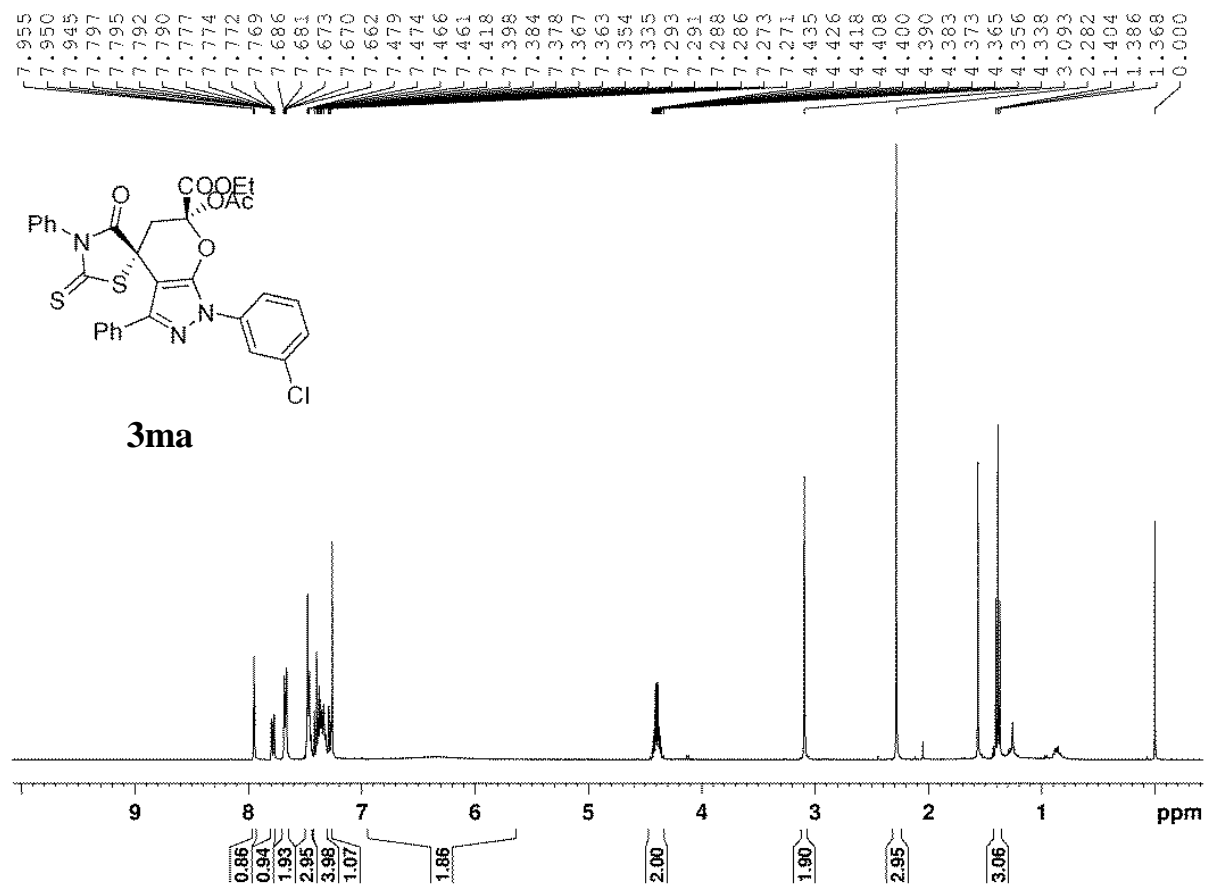

${ }^{13} \mathrm{C}$-NMR spectrum of $\mathbf{3 m a}$ in $\mathrm{CDCl}_{3}(100 \mathrm{MHz})$

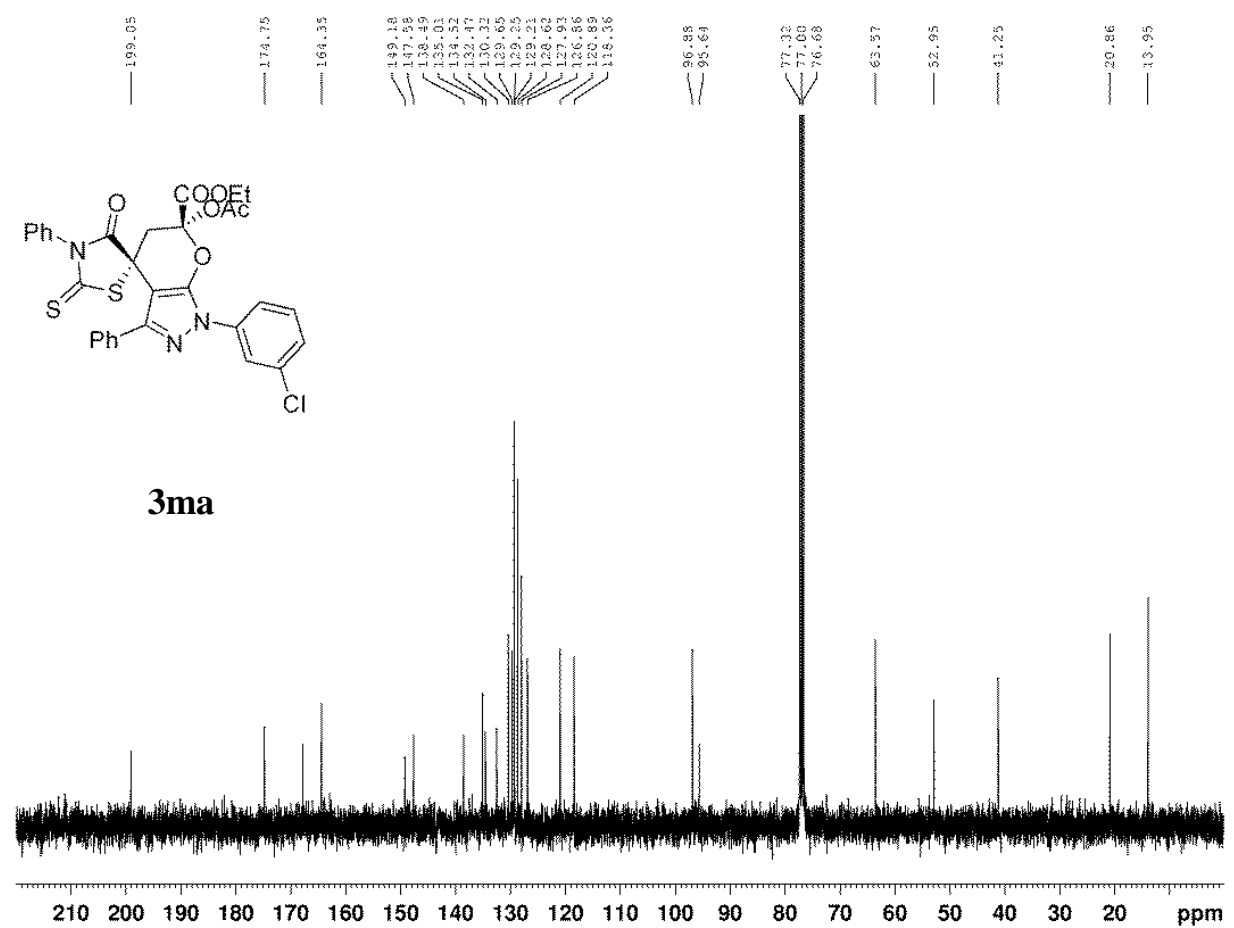


${ }^{1} \mathrm{H}-\mathrm{NMR}$ spectrum of $\mathbf{3 n a}$ in $\mathrm{CDCl}_{3}(600 \mathrm{MHz})$

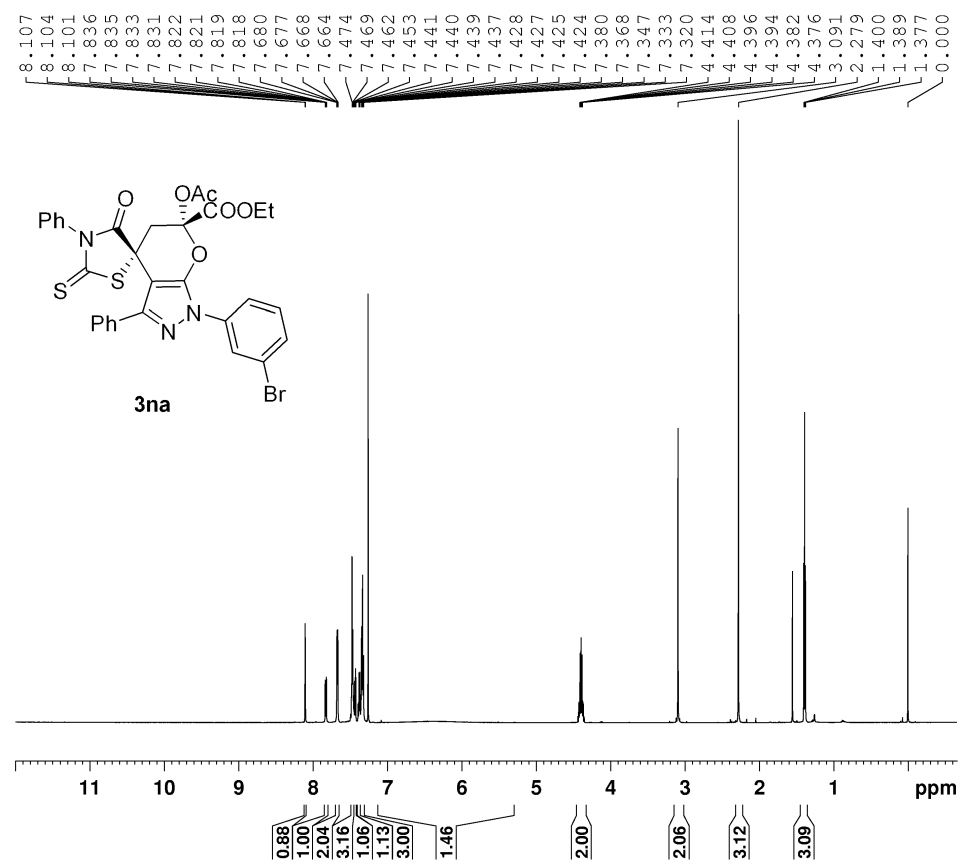

${ }^{13} \mathrm{C}-\mathrm{NMR}$ spectrum of 3na in $\mathrm{CDCl}_{3}(150 \mathrm{MHz})$
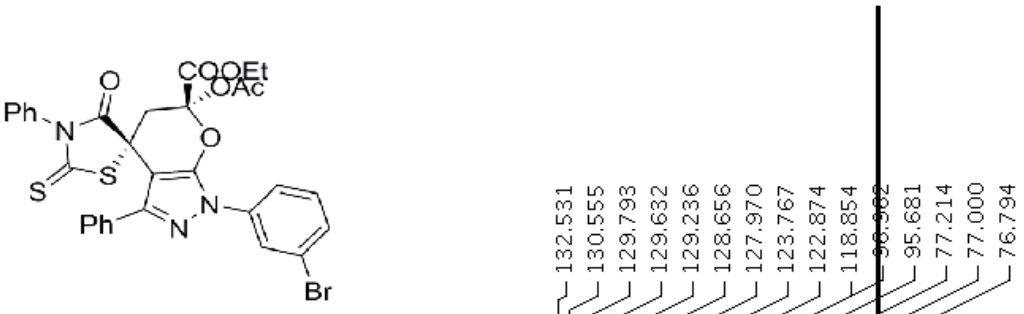

3na
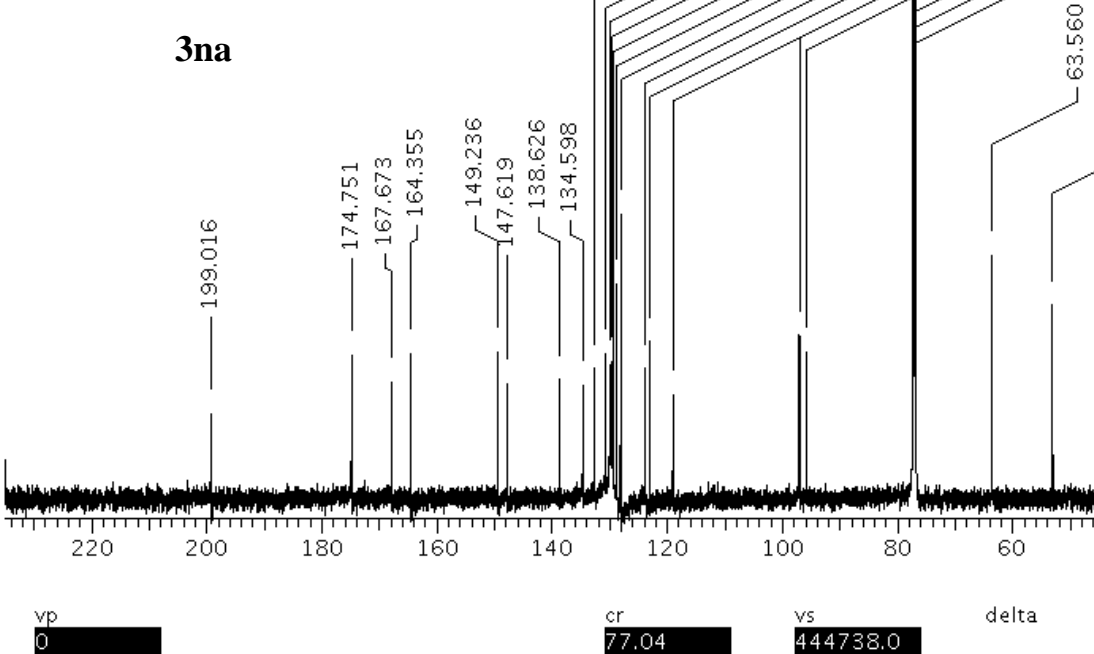

兽

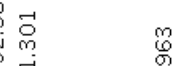

$\int \vec{y}$ 
${ }^{1} \mathrm{H}-\mathrm{NMR}$ spectrum of $\mathbf{3 o a}$ in $\mathrm{CDCl}_{3}(600 \mathrm{MHz})$

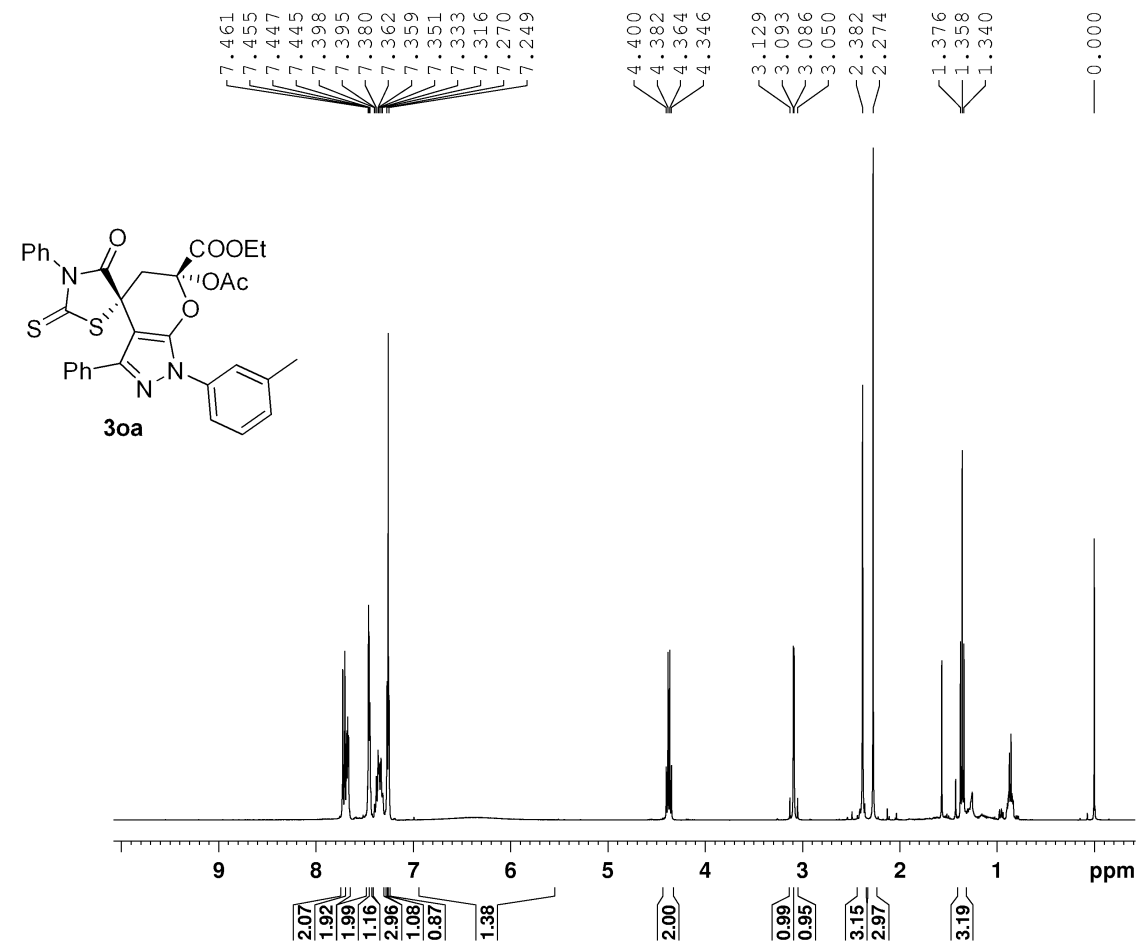

${ }^{13} \mathrm{C}$-NMR spectrum of $30 a$ in $\mathrm{CDCl}_{3}(150 \mathrm{MHz})$

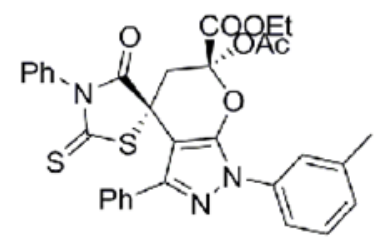

$30 a$

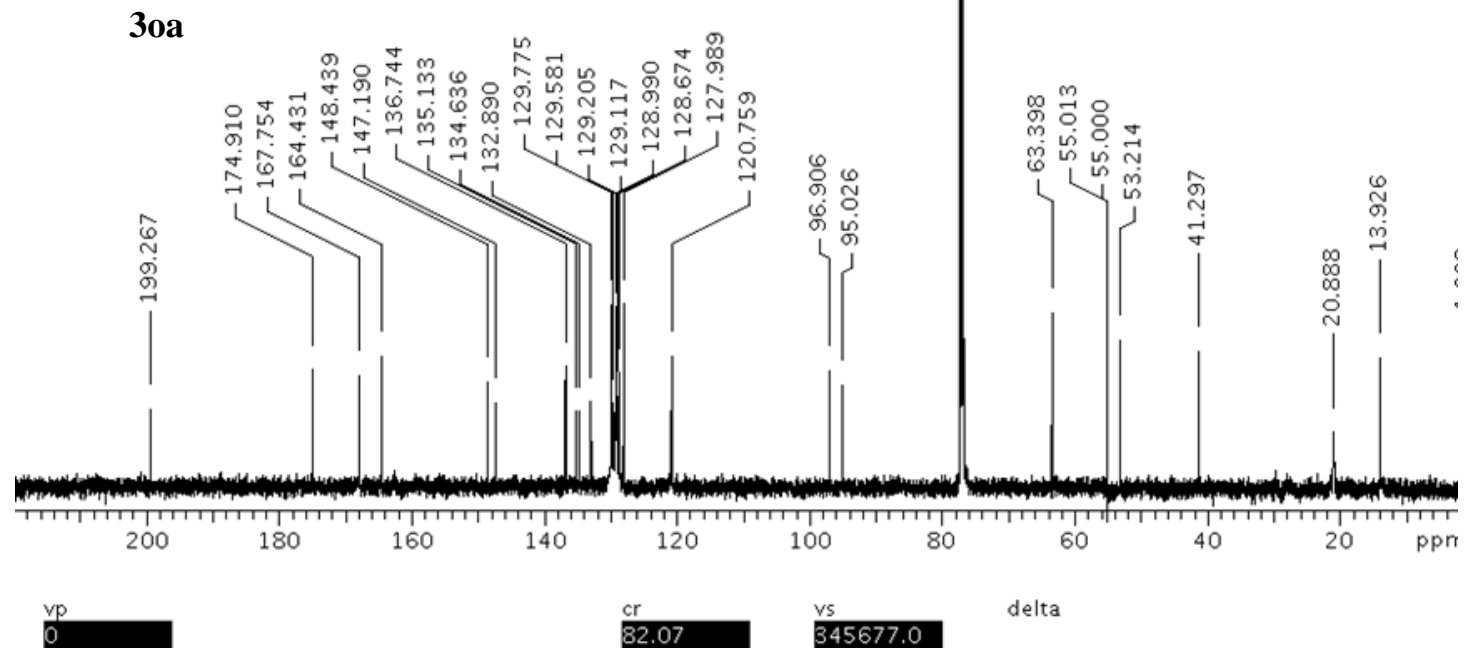


${ }^{1} \mathrm{H}-\mathrm{NMR}$ spectrum of $\mathbf{3 p a}$ in $\mathrm{CDCl}_{3}(400 \mathrm{MHz})$

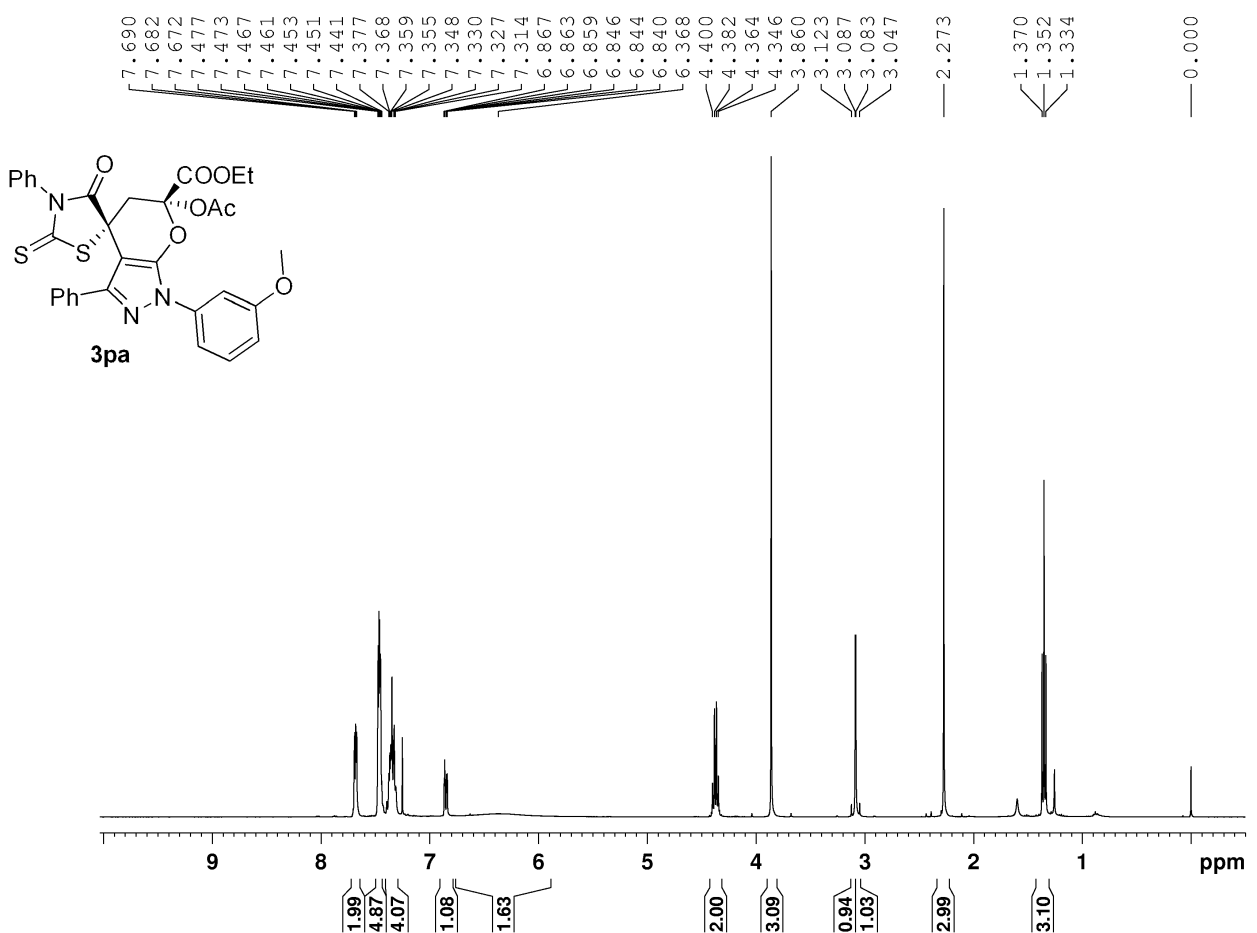

${ }^{13} \mathrm{C}-\mathrm{NMR}$ spectrum of $\mathbf{3 p a}$ in $\mathrm{CDCl}_{3}(100 \mathrm{MHz})$

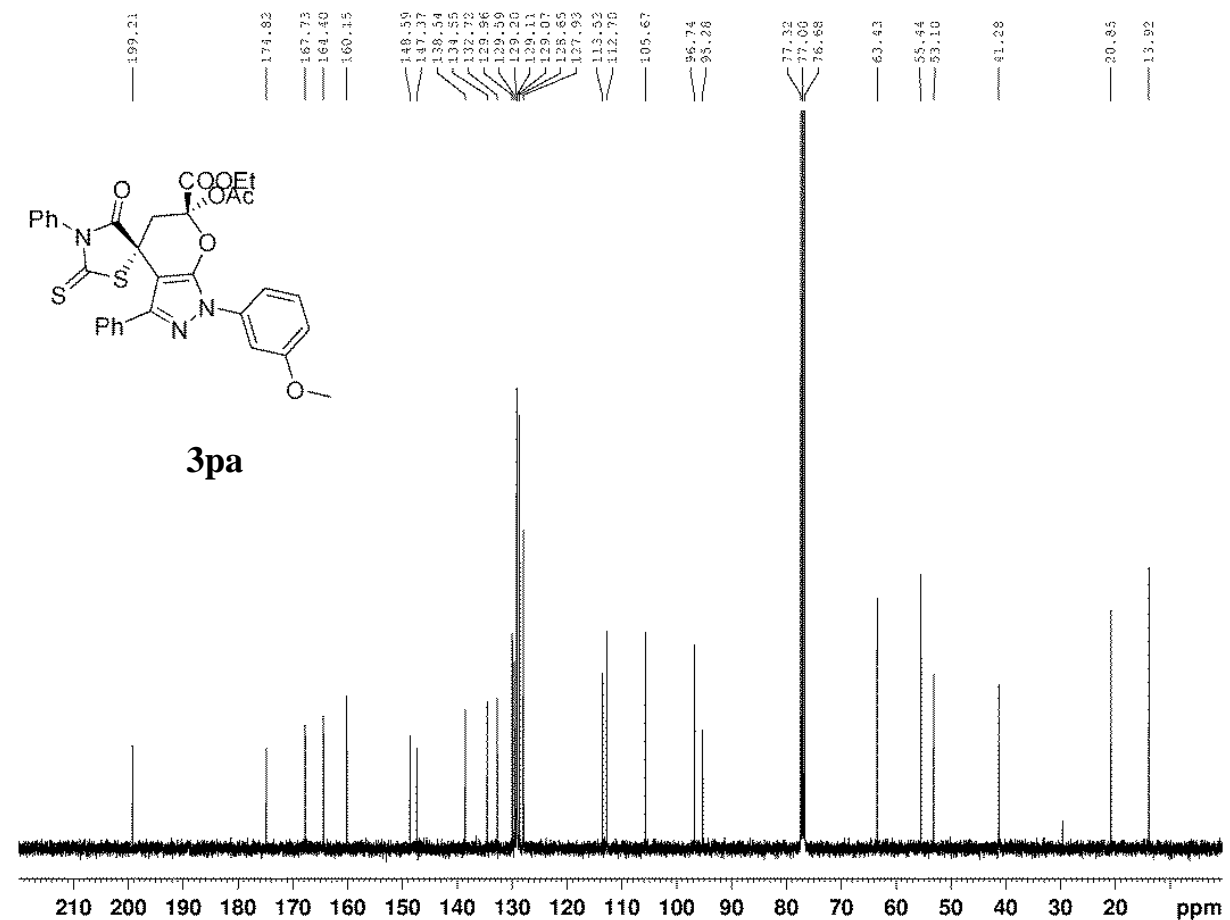


${ }^{1} \mathrm{H}-\mathrm{NMR}$ spectrum of $\mathbf{3 q a}$ in $\mathrm{CDCl}_{3}(400 \mathrm{MHz})$
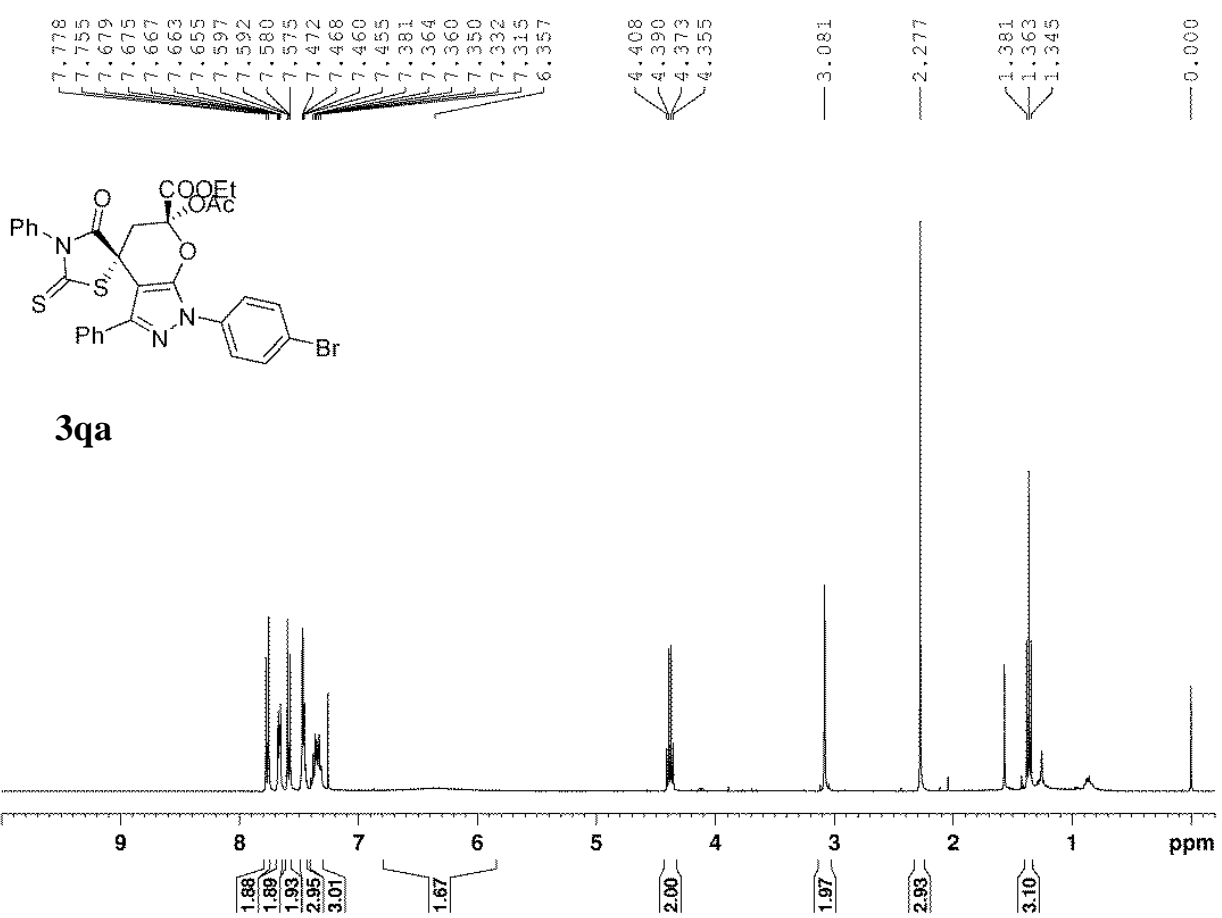

${ }^{13} \mathrm{C}-\mathrm{NMR}$ spectrum in of $\mathbf{3 q a} \mathrm{CDCl}_{3}(100 \mathrm{MHz})$

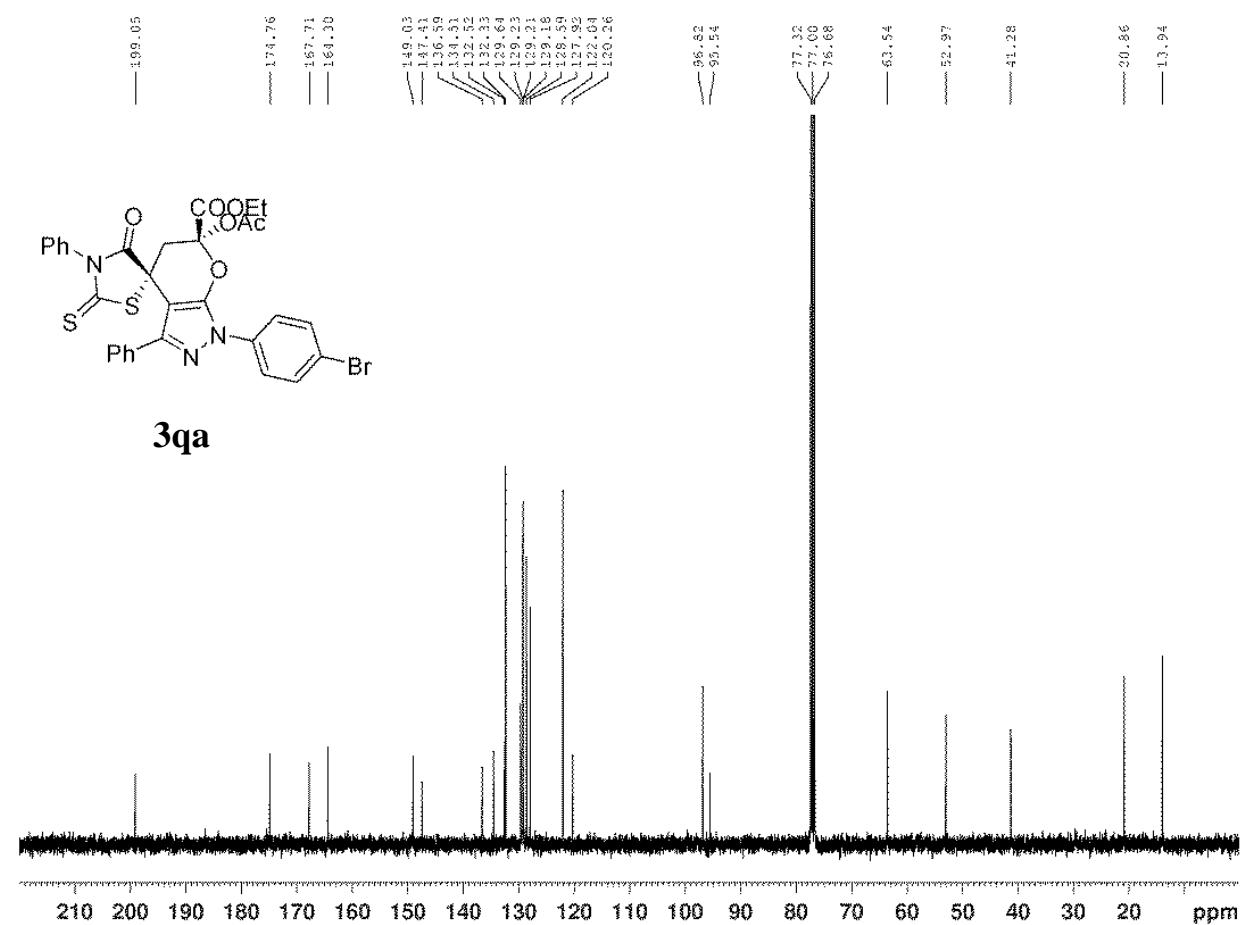


${ }^{1} \mathrm{H}-\mathrm{NMR}$ spectrum in of $\mathbf{3 r a ~} \mathrm{CDCl}_{3}(400 \mathrm{MHz})$

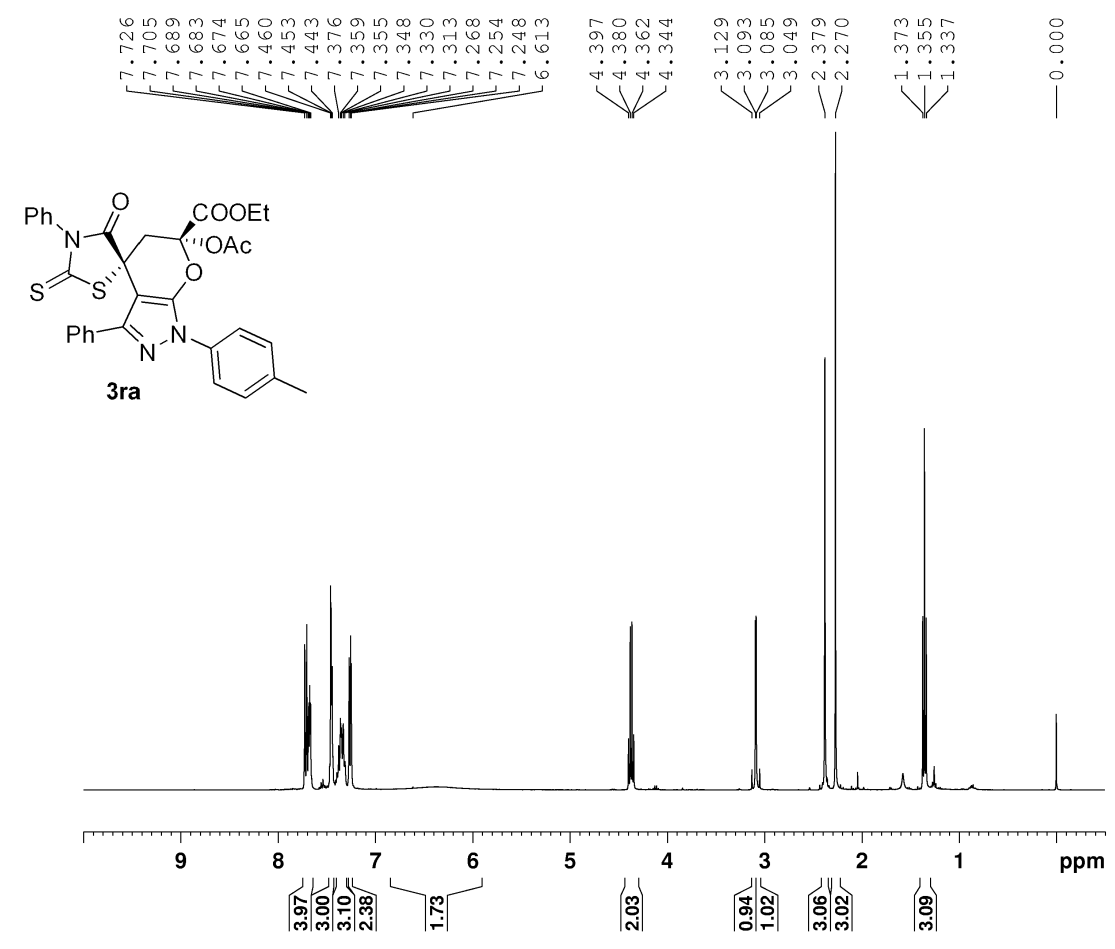

${ }^{13} \mathrm{C}-\mathrm{NMR}$ spectrum of 3ra in $\mathrm{CDCl}_{3}(100 \mathrm{MHz})$

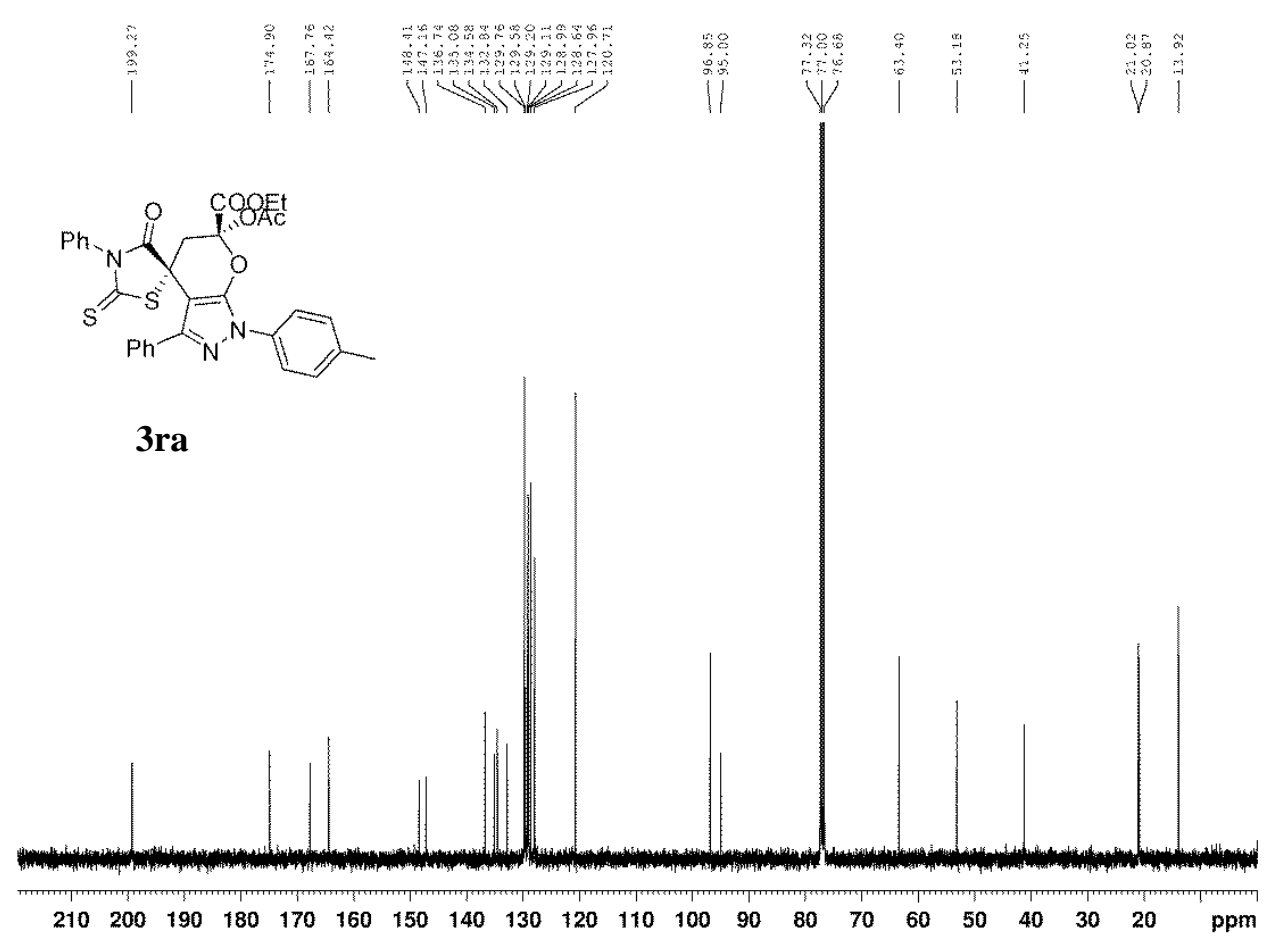


${ }^{1} \mathrm{H}-\mathrm{NMR}$ spectrum of 3sa in $\mathrm{CDCl}_{3}(600 \mathrm{MHz})$

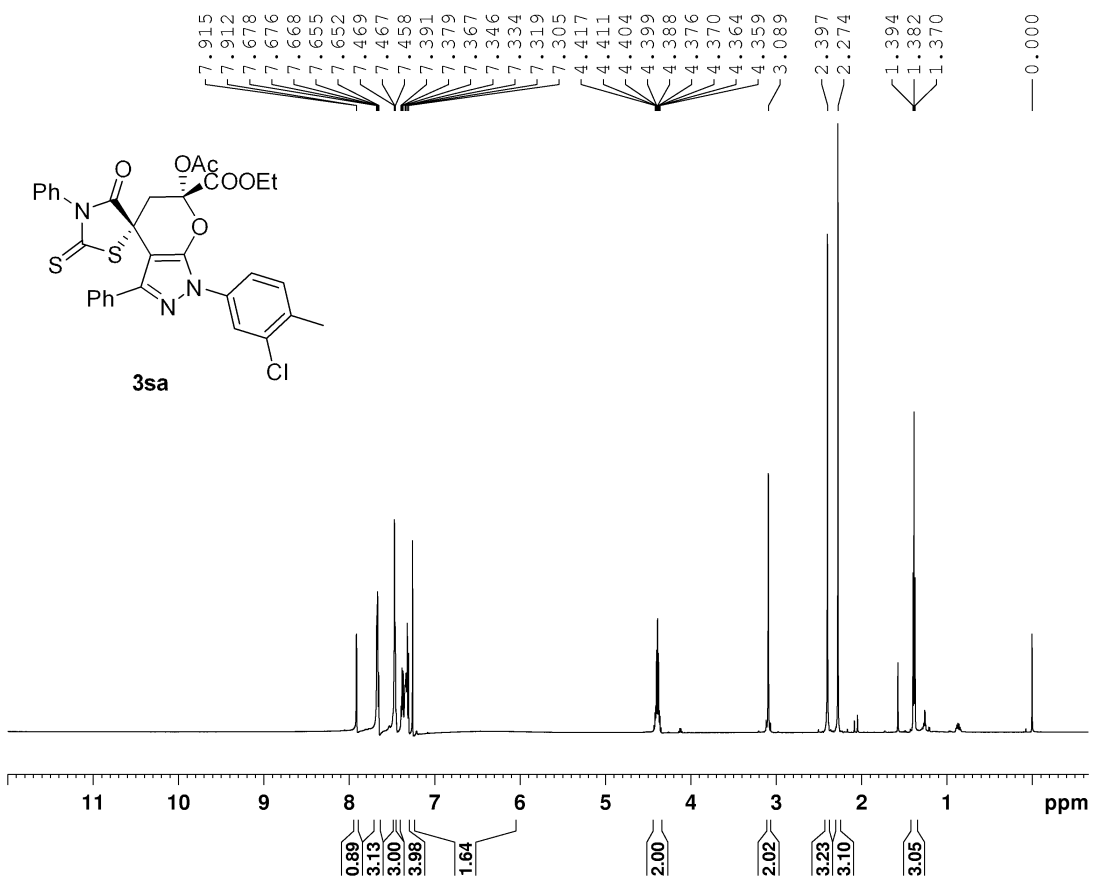

${ }^{13} \mathrm{C}-\mathrm{NMR}$ spectrum of $\mathbf{3 s a}$ in $\mathrm{CDCl}_{3}(150 \mathrm{MHz})$

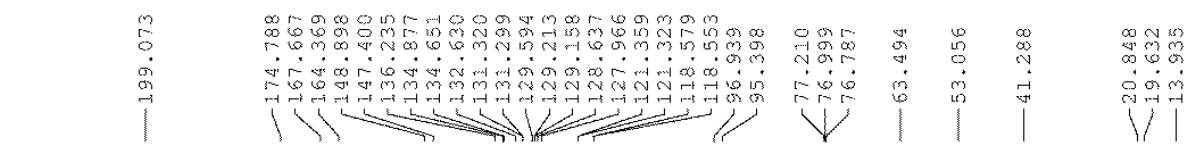<smiles>CCOC(=O)C1(C(=O)OCC)C[C@@]2(SC(=S)N(c3ccccc3)C2=O)c2c(-c3ccccc3)nn(-c3ccc(C)c(Cl)c3)c21</smiles>

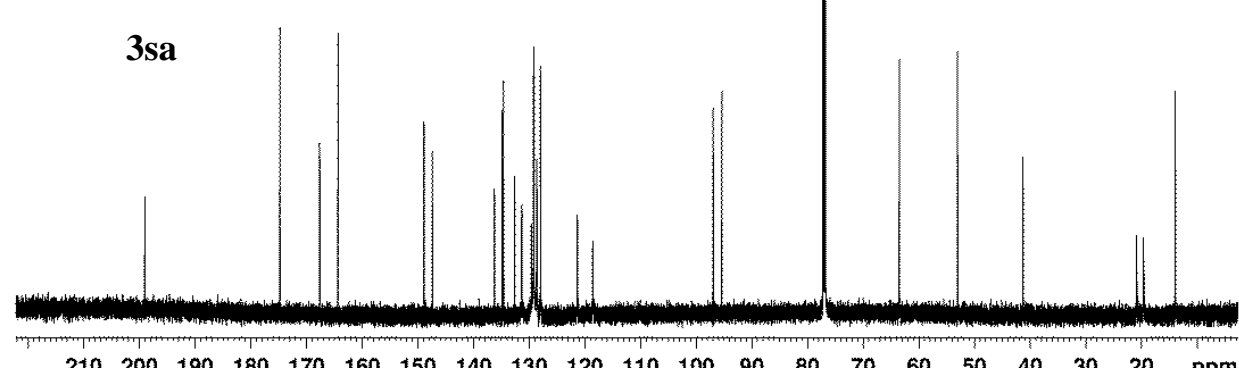


${ }^{1} \mathrm{H}-\mathrm{NMR}$ spectrum of $\mathbf{4 a a}$ in $\mathrm{CDCl}_{3}(400 \mathrm{MHz})$

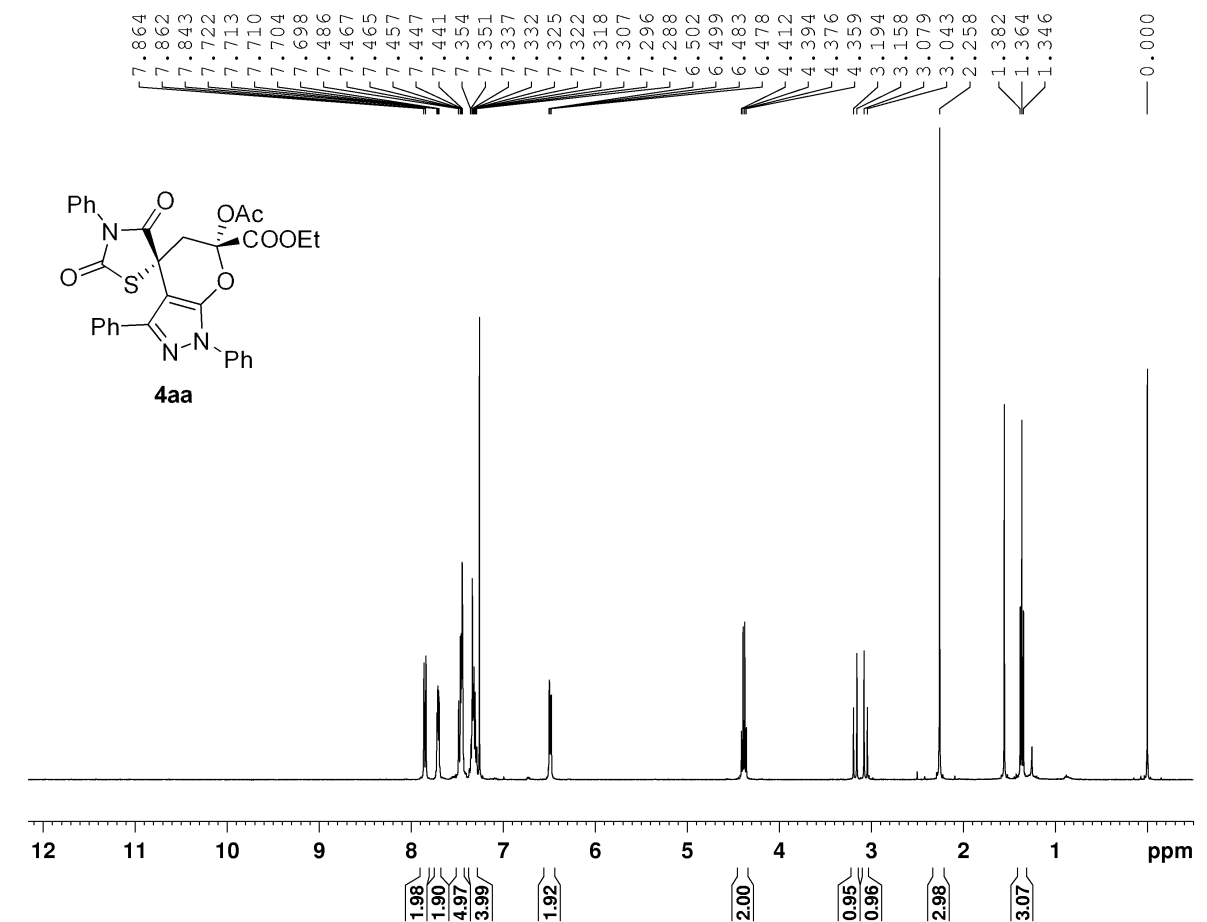

${ }^{13} \mathrm{C}-\mathrm{NMR}$ spectrum of $\mathbf{4 a a}$ in $\mathrm{CDCl}_{3}(100 \mathrm{MHz})$

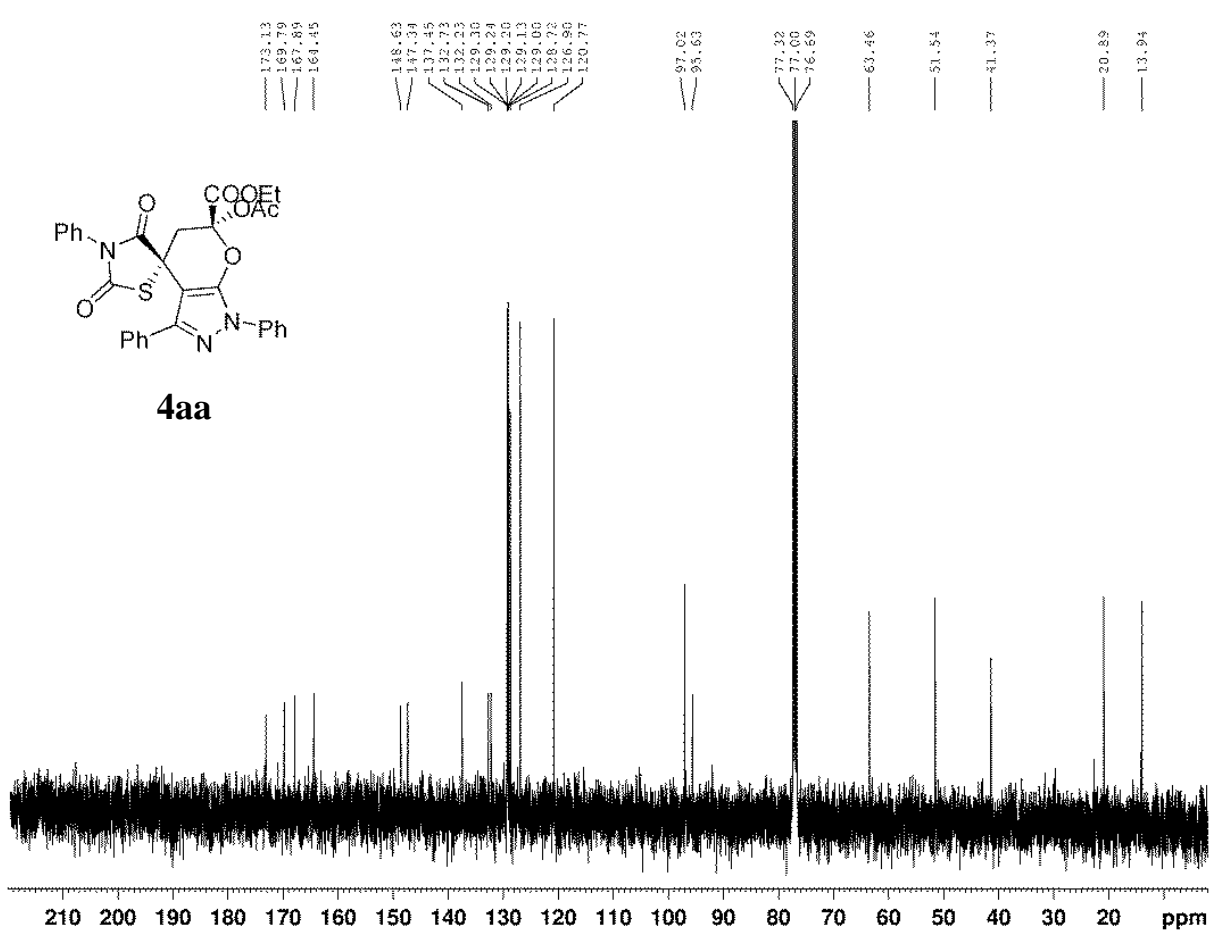


${ }^{1} \mathrm{H}-\mathrm{NMR}$ spectrum of $\mathbf{1 a}$ in $\mathrm{CDCl}_{3}(400 \mathrm{MHz})$

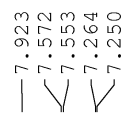

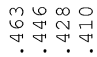<smiles>CCOC(=O)/C=C1/SC(=S)N(c2ccccc2)C1=O</smiles>

$1 a$

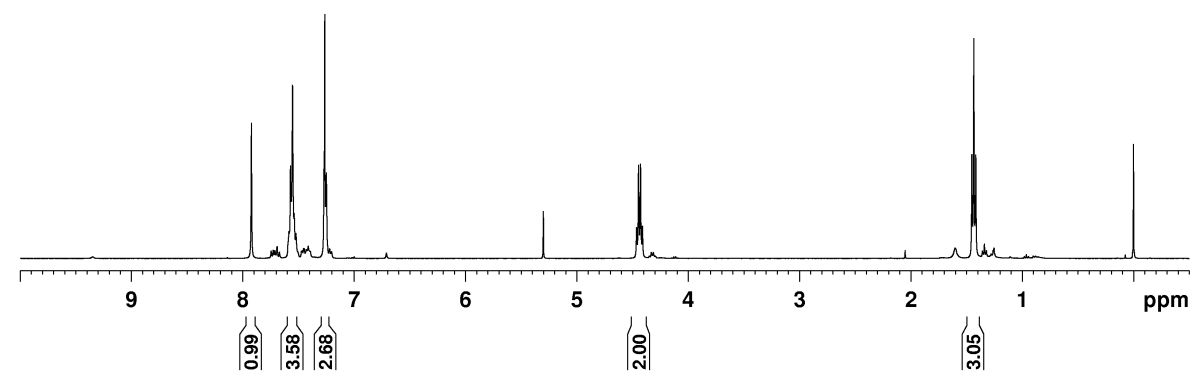

${ }^{13} \mathrm{C}-\mathrm{NMR}$ spectrum of $\mathbf{1 a}$ in $\mathrm{CDCl}_{3}(100 \mathrm{MHz})$
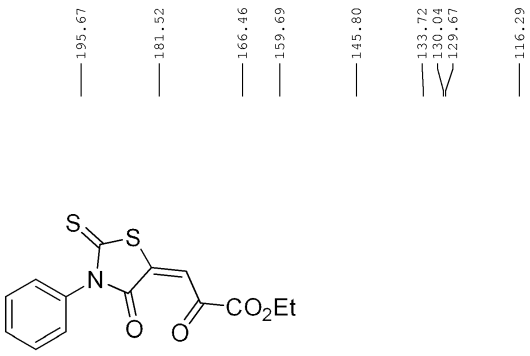

$1 a$

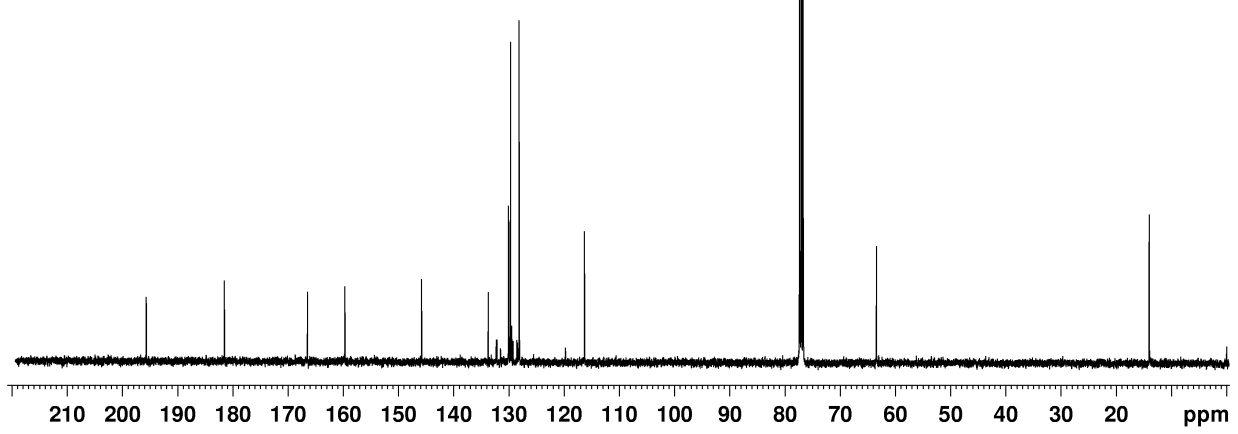


${ }^{1} \mathrm{H}-\mathrm{NMR}$ spectrum of $\mathbf{1 b}$ in $\mathrm{CDCl}_{3}(400 \mathrm{MHz})$

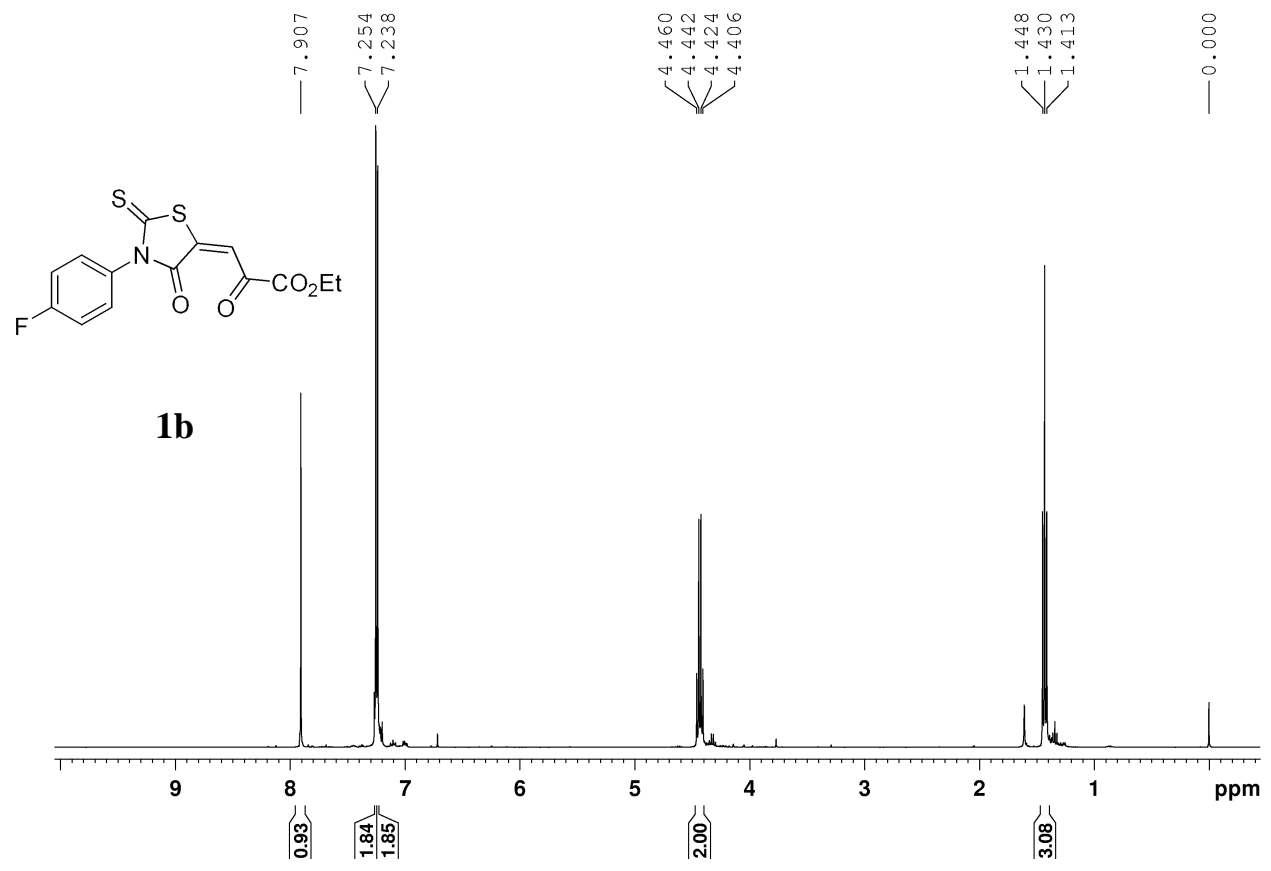

${ }^{13} \mathrm{H}-\mathrm{NMR}$ spectrum of $\mathbf{1 b}$ in $\mathrm{CDCl}_{3}(100 \mathrm{MHz})$

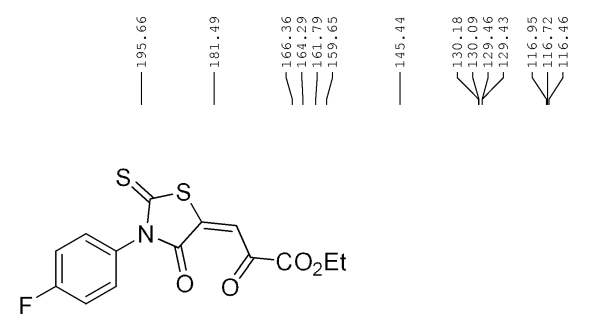

$1 \mathbf{b}$

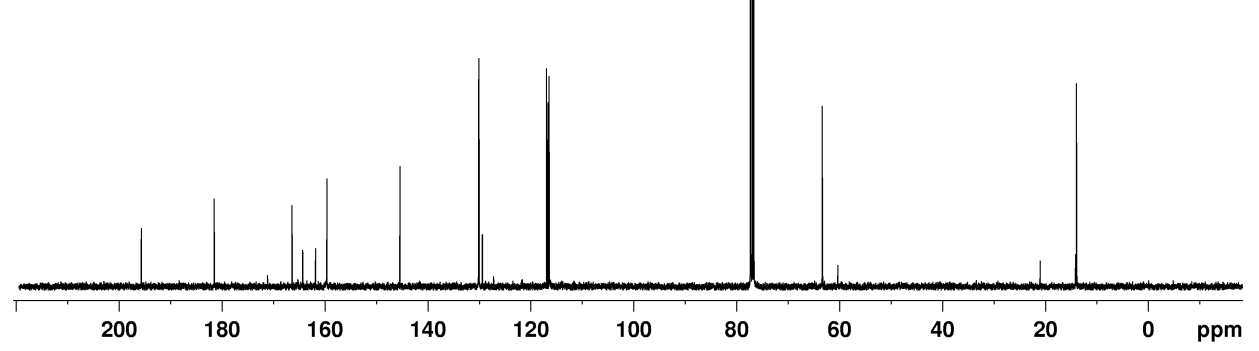


${ }^{1} \mathrm{H}-\mathrm{NMR}$ spectrum of $\mathbf{1 c}$ in $\mathrm{CDCl}_{3}(400 \mathrm{MHz})$

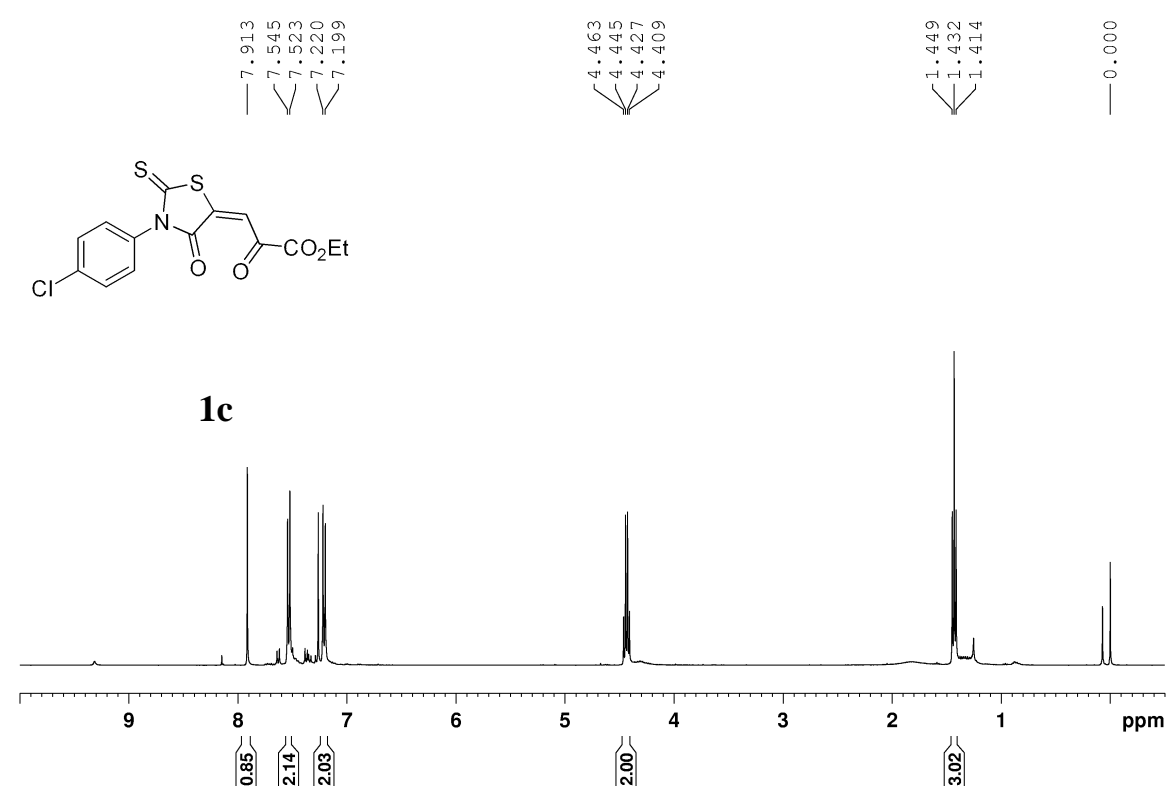

${ }^{13} \mathrm{C}$-NMR spectrum of $\mathbf{1 c}$ in $\mathrm{CDCl}_{3}(100 \mathrm{MHz})$

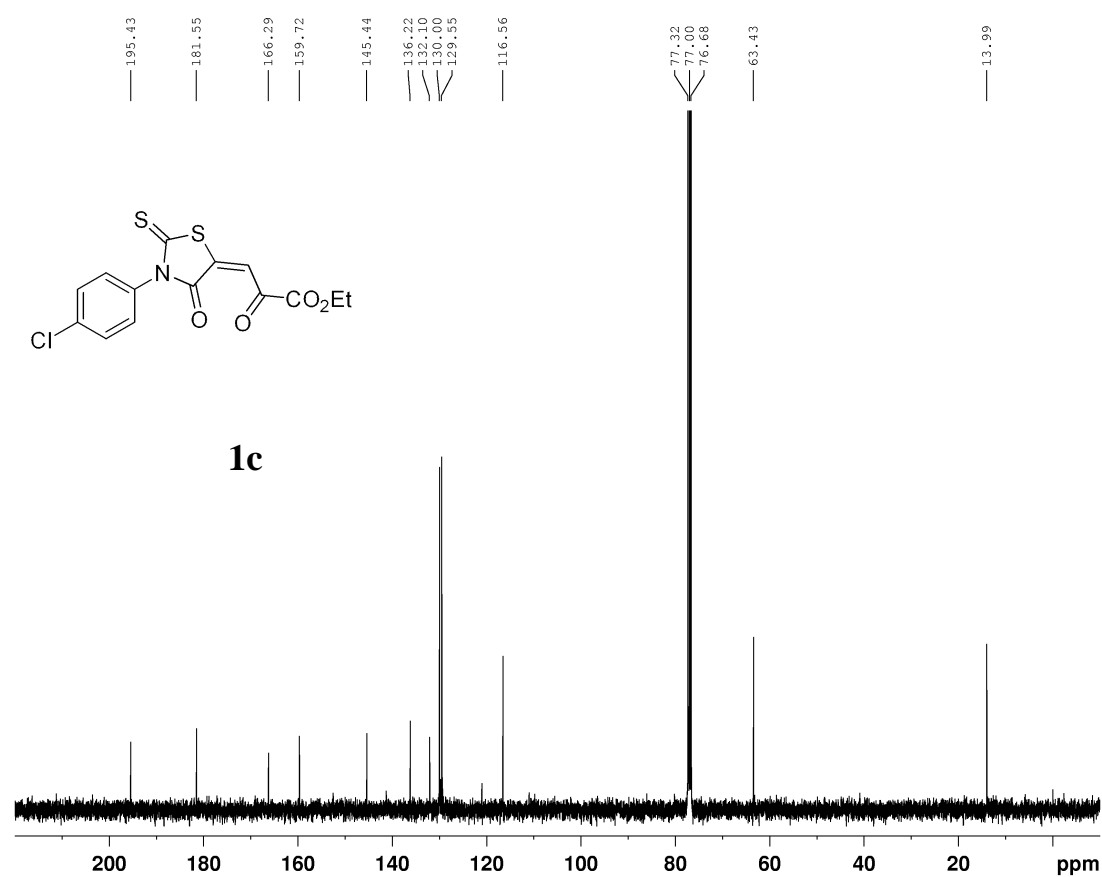


${ }^{1} \mathrm{H}-\mathrm{NMR}$ spectrum of $\mathbf{1 d}$ in $\mathrm{CDCl}_{3}(400 \mathrm{MHz})$

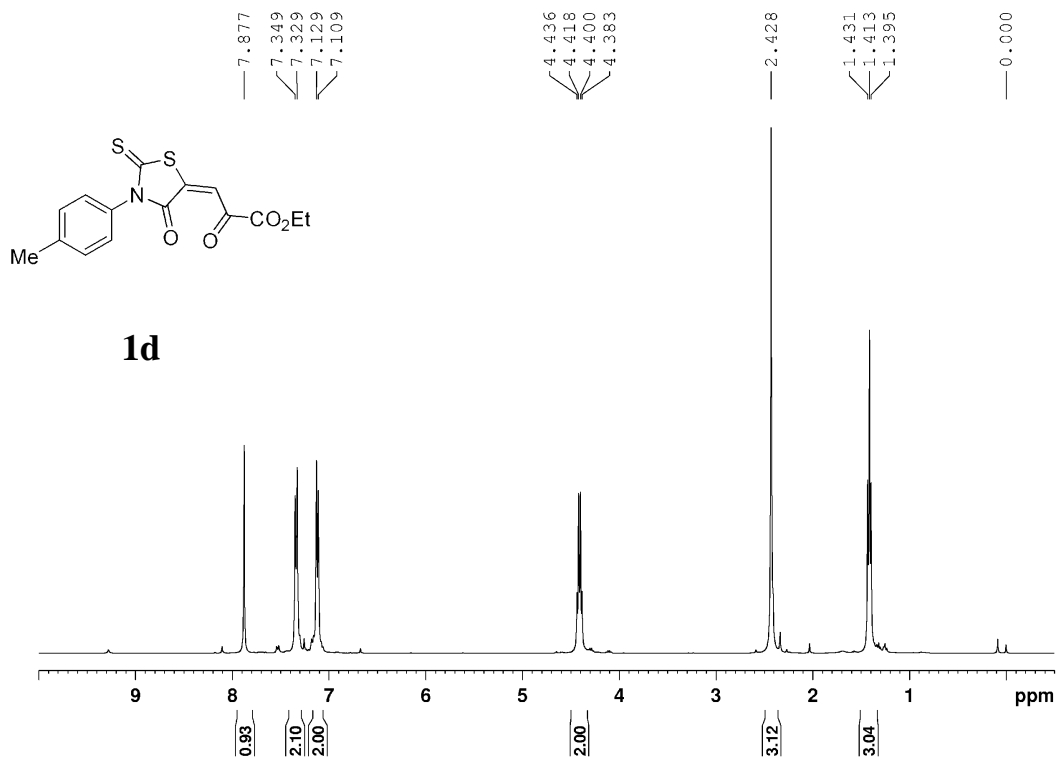

${ }^{13} \mathrm{C}-\mathrm{NMR}$ spectrum of $\mathbf{1 d}$ in $\mathrm{CDCl}_{3}(100 \mathrm{MHz})$

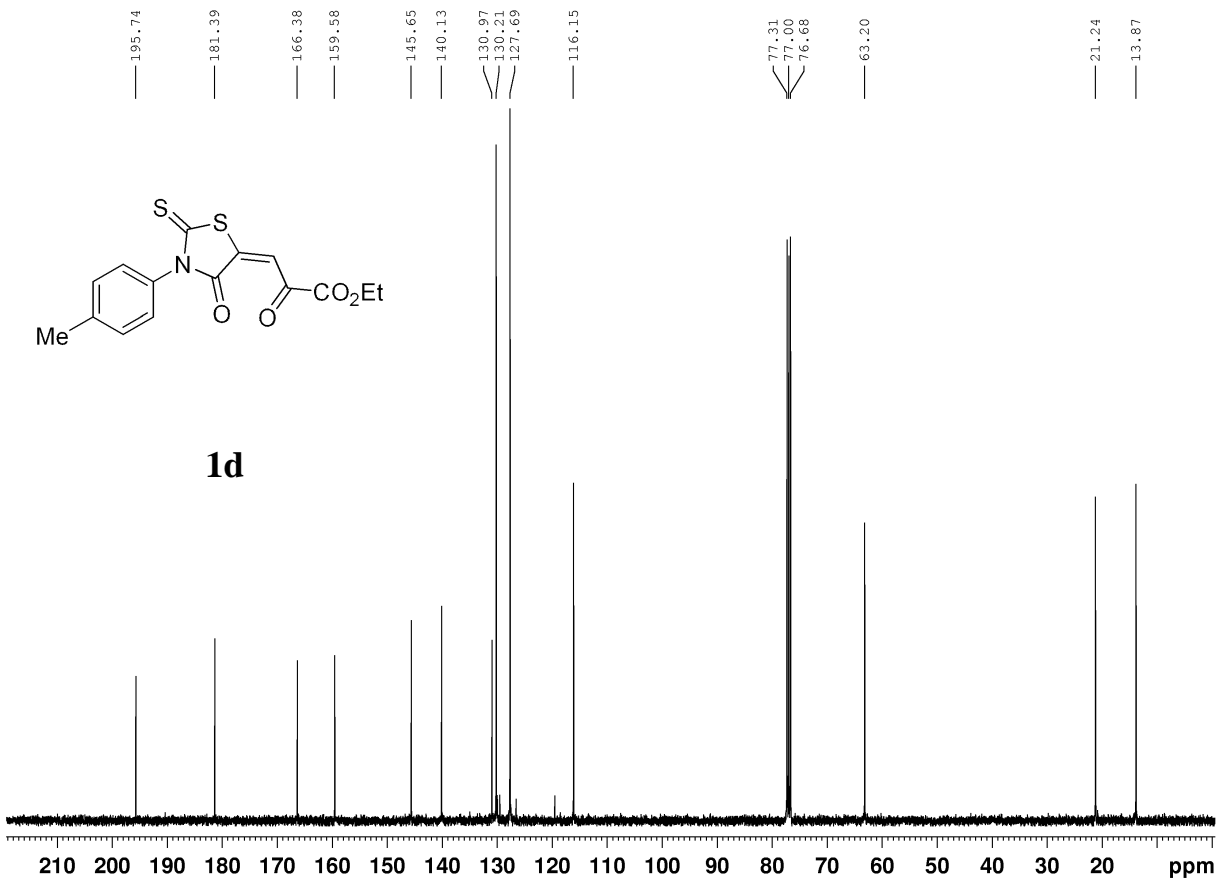


${ }^{1} \mathrm{H}-\mathrm{NMR}$ spectrum of $\mathbf{1 e}$ in $\mathrm{CDCl}_{3}(400 \mathrm{MHz})$

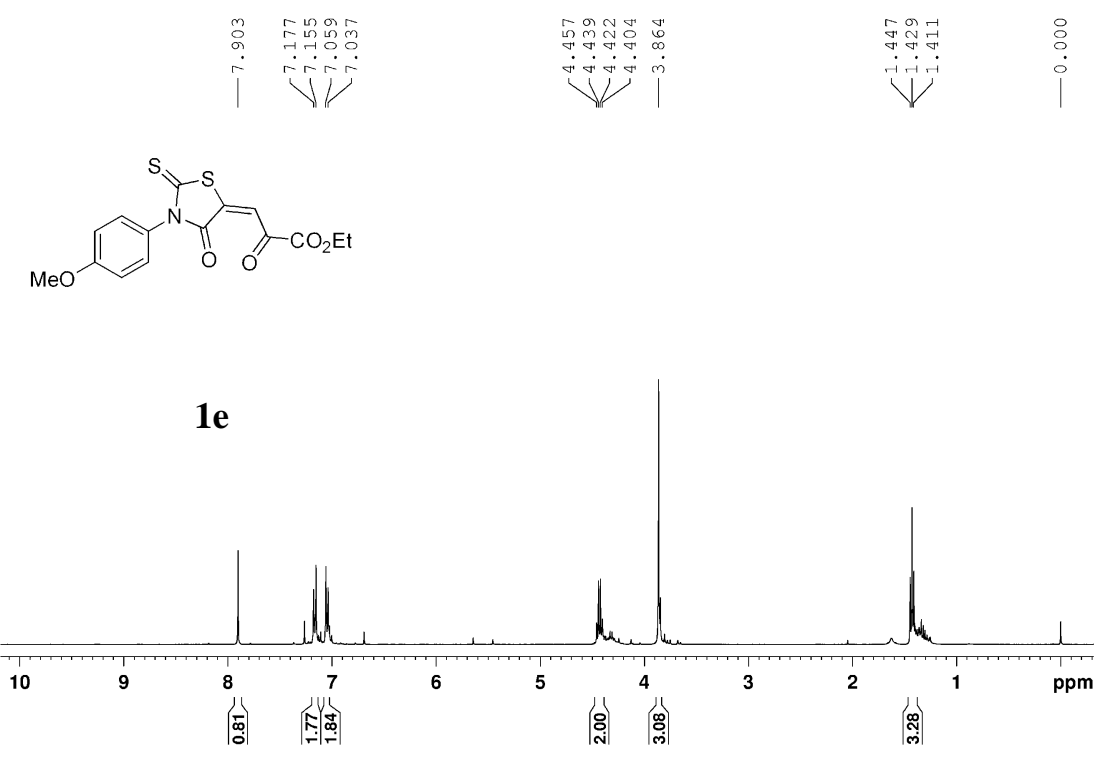

${ }^{13} \mathrm{C}-\mathrm{NMR}$ spectrum of $\mathbf{1 e}$ in $\mathrm{CDCl}_{3}(100 \mathrm{MHz})$

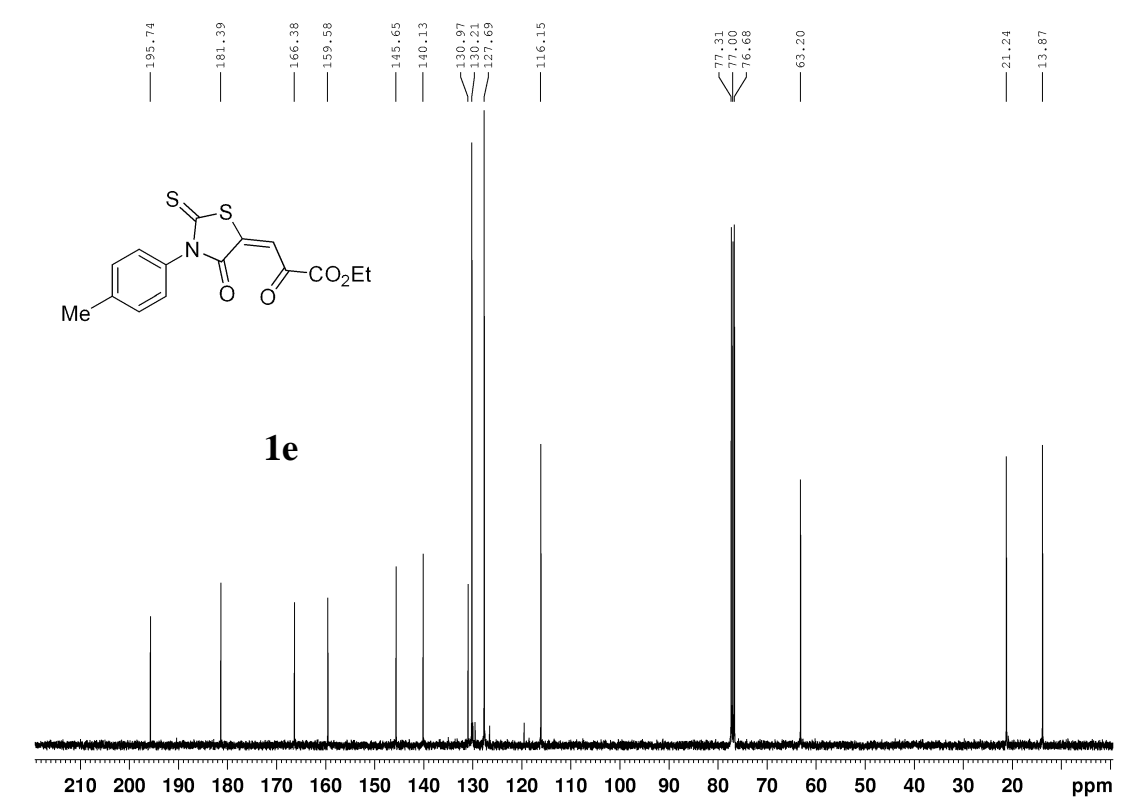

